Carotid Intima-Media Thickness and Cardiovascular Disease Risk in African Populations

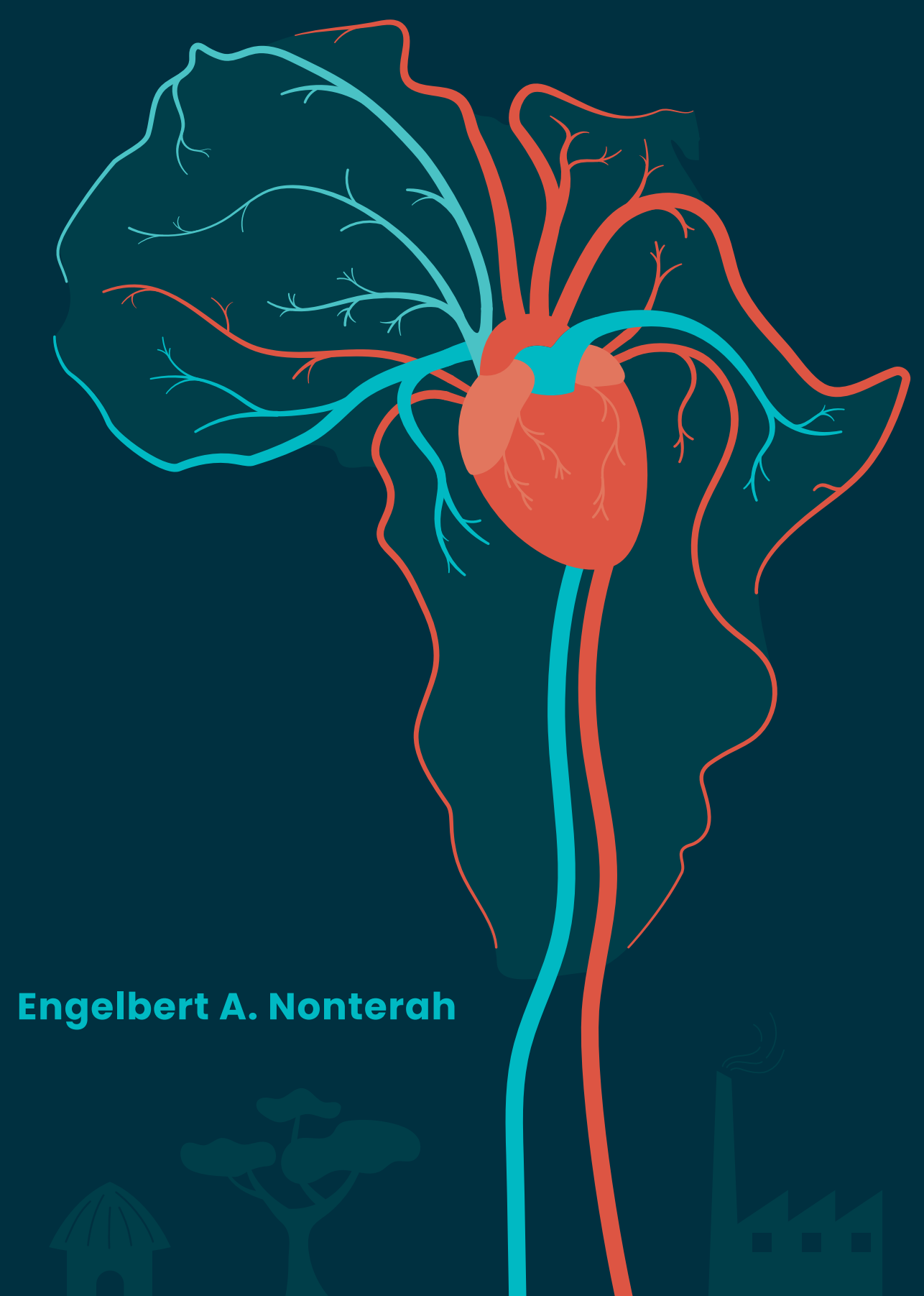





\title{
Carotid intima-media thickness and cardiovascular disease risk in African populations
}

\author{
Engelbert A. Nonterah
}


Author: Engelbert A. Nonterah

Provided by thesis specialist Ridderprint, ridderprint.nl

Printing: Ridderprint

Layout \& cover design: Sara Terwisscha van Scheltinga, persoonlijkproefschrift.nl

ISBN: 978-94-6416-876-1

Electronic ISBN: 978-94-6416-873-0

\section{Copyright $2021 \odot$ Engelbert A. Nonterah}

The Netherlands. Copyrights of articles that have been published or have been accepted for publication have been transferred to the respective journals.

Financial support for this by the Global Health Scholarship at the Julius Center for Health Sciences and Primary Care, University Medical Center Utrecht, Utrecht University, the Netherlands is duly acknowledged. The author wishes to appreciate the various support received from the Navrongo Health Research Center. 


\title{
Carotid intima-media thickness and cardiovascular disease risk in African populations
}

\author{
De wanddikte van de halsslagader als indicator voor het hartvaatziekte \\ risico in de Afrikaanse bevolking \\ (met een samenvatting in het Nederlands)
}

\section{Proefschrift}

ter verkrijging van de graad doctor aan de

Universiteit Utrecht

op gezag van de

rector magnificus, prof. dr. H.R.B.M. Kummeling, ingevolge het besluit van het college voor promoties in het openbaar te verdedigen op

dinsdag 7 december 2021 des middags te 2.15 uur

$$
\text { door }
$$

\section{Engelbert Adamwaba Nonterah}

geboren op 10 februari 1984 te Navrongo, Ghana 


\section{Promotoren:}

Prof. dr. D.E. Grobbee

Prof. dr. N.J. Crowther

\section{Copromotoren:}

Dr. K. Klipstein-Grobusch

Dr. A.R. Oduro 


\section{Table of contents}

Chapter 1

General introduction

\section{Part 1: Risk factors for CIMT}

\section{Chapter 2}

Classical cardiovascular risk factors and HIV are associated with Carotid intima-media thickness in adults from sub-Saharan Africa: Findings from H3Africa AWI-Gen study

\section{Chapter 3}

Adiposity phenotypes and subclinical atherosclerosis in adults from subSaharan Africa: An H3Africa AWI-Gen study

\section{Chapter 4}

Race-ethnic differences in the association between classical cardiovascular risk factors and common carotid intima-media thickness: an individual participant data meta-analysis

\section{Part 2: CIMT and cardiovascular disease risk}

\section{Chapter 5}

Poor cardiovascular health is associated with subclinical atherosclerosis in apparently healthy sub-Saharan African populations: an H3Africa AWI-Gen study

\section{Chapter 6}

Carotid atherosclerosis, microalbuminuria and predicted 10-year cardiovascular disease risk in sub-Saharan Africa: An H3Africa AWI-Gen study

\section{Chapter 7}

Discussion 


\section{Global burden of cardiovascular disease}

The global burden of cardiovascular disease (CVD) and associated mortality is increasing due to ageing and population growth (1-3). Particularly in low- and middle-income countries (LMIC) urbanization and globalization of communities with resultant socio-economic development, has led to lifestyle changes that increase the risk for CVDs. The current Global Burden of Disease study reports that the total CVD cases such as ischemic heart disease, myocardial infarction, cerebrovascular accidents (stroke), heart failure, cardiomyopathies and peripheral vascular diseases nearly doubled from 271 million in 1990 to 523 million in 2019. The number of CVD deaths experienced a steady increase from 12.1 million to 18.6 million in the same period (2). The 2019 estimates ranked ischaemic heart disease and stroke as the top cause of disability adjusted-life years (DALYS) in the older age groups (50-74 years and above) $(2,3)$. Presented in Figure 1 is the data from Roth et al (2020), presenting age standardised change in CVD deaths from 2010-2019, number of deaths from 1990-2019 and proportions of CVD deaths by cause in 2019 (3).

From 1990 to 2013 there was a 40\% increase in global CVD deaths of which ageing accounted for $55 \%$ while population growth accounted for $25 \%$ of these deaths (1). The World Health Organization (WHO) suggests that by 2030 there would be substantial increases in CVD globally and especially in the African region.

Aetiologically, CVDs arise from a complex interaction between genetic, lifestyle and metabolic risk, access to treatment in acute phase of CVD and advanced management or secondary prevention for established CVD. Over the years several longitudinal studies using clinical endpoints of CVD identified the major risk factors for these diseases. Starting with the Framingham study which began 73 years ago (4) to the updated 2018 Pooled Cohort Equation (PCE) study (5), the following have been established as the main risk factors for CVD: age, sex, smoking, elevated blood pressure, increased body mass index, unfavourable lipid profile, glucose intolerance and physical inactivity. The American Heart Association and the Global Burden of Disease Study further identified these risk factors as well as high alcohol intake as the main contributors to the burden of CVD (6). The development of the PCE was based on data from the ARIC study, Cardiovascular Health Study, Coronary Artery Risk Development in Young Adults, the Framingham Heart Study offspring cohort, the Jackson Heart Study and the Multi-Ethnic study of Atherosclerosis (5). 
Percent Change in Age-Standarized CVD Death Rate from 2010-2019

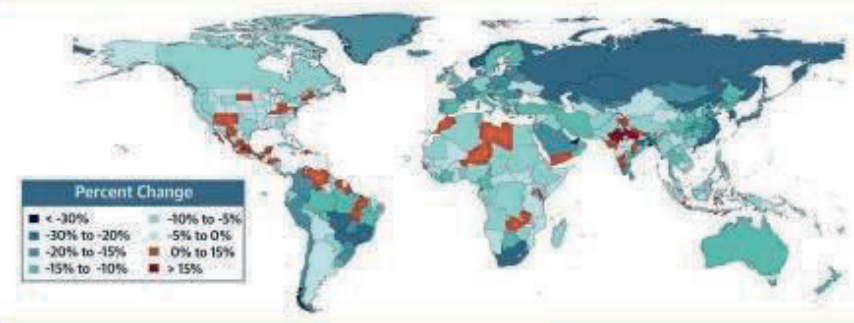

Number of CVD Deaths
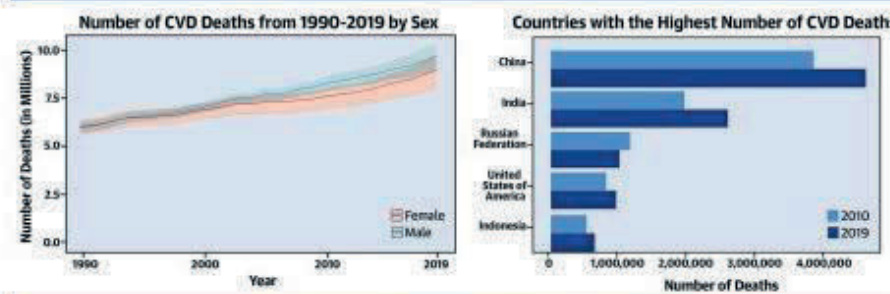

Proportion of CVD Deaths by Cause (2019)

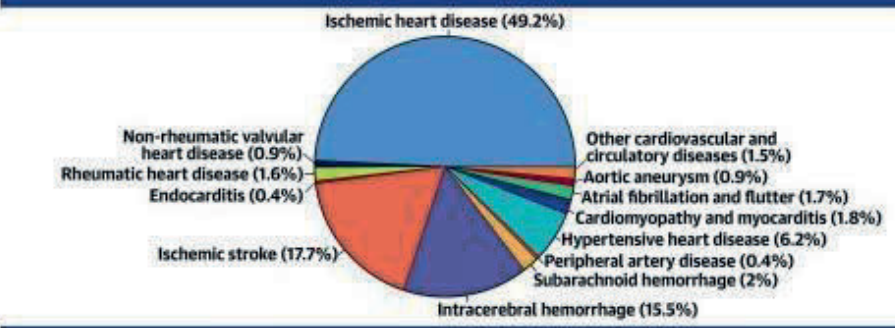

CVD Burden Attributable to Modifiable Risk Factors

Figure 1: Age standardised change in CVD deaths from 2010-2019, number of deaths from 1990-2019 and proportions of CVD deaths by cause in 2019 (Roth et al 2020 (3))

Despite the high global burden of disease there are observed regional differences in the levels of CVD morbidity and mortality. These differences are attributed to several factors broadly categorized as:

- Factors that affect risk of CVD and are targets for prevention, mainly lifestyle and metabolic risk factors.

- Those that affect survival in the acute phase hinging on the social determinants of health such as access to health care in the acute phase and available treatment options.

- Factors that affect secondary prevention such as access to advanced pharmacological and non-pharmacological treatment options. 
The contribution of genetics and novel risk factors such as microalbuminuria and inflammation as well as socio-cultural factors is also important.

The projected global increase in the prevalence of CVD $(1,2)$ will have significant clinical and public health implications for many countries, especially those with poor healthcare infrastructure. Formulation of context specific interventions is therefore essential to mitigate the rising burden of CVD.

\section{Cardiovascular Disease in sub-Saharan Africa}

Low- and middle-income countries such as those in sub-Saharan Africa (SSA) suffer a disproportionately higher burden of CVD and premature mortality due to CVD. For example, nearly $80 \%$ of global CVD deaths occur in these countries (3). These countries are rapidly undergoing a transition from communicable diseases to non-communicable diseases (7). However, the co-occurrence of infectious diseases such as HIV, tuberculosis and malaria creates a double burden of diseases (7) further posing huge challenges to healthcare delivery through rising healthcare cost as well as cost related to integrated chronic disease care.

The epidemiology of CVD in SSA is different from that in other regions of the world. CVD risk factor levels in SSA are generally increasing but still remain low compared to high income countries (8). For instance, obesity and hypertension levels are high in most African countries (9-12). CVD mortality is higher in younger age groups when compared to other regions of the world (8). This leads to a loss of persons in the economically viable age groups, and therefore has effects on the economic status of the country.

African populations are experiencing an increase in the proportion of older subjects due to improved life expectancies (13). In addition, increased socioeconomic growth and movements of populations to urban centers within these countries has resulted in changes in lifestyles which have led to the increased consumption of processed, energy-dense foods and high levels of physical inactivity (14).

The co-occurrence of rising CVD risk with persistently high chronic infectious diseases (such as HIV, tuberculosis and malaria) further escalates the unfolding CVD burden $(15,16)$. These diseases are strongly linked to CVD risk. Immune activation from active HIV is known to induce sustained inflammation which can exacerbate atherosclerotic processes $(17,18)$. With the advent of antiretroviral therapy (ART), the HIV population is also undergoing the normal ageing process due to reduced HIV/AIDS related deaths, exposing them to greater risk for CVD. In addition, the early generations of ARTs were known to cause several lipid 
abnormalities that increased CVD risk (17). The newer generation of medication has reduced this occurrence and further improved life expectancies. However, newer drugs such as dolutegravir used commonly in SSA are reported to be associated with obesity and hyperglycaemia $(19,20)$. The ART care cascade has also served as a good avenue for the screening and management of CVD and their risk $(21,22)$.

Other factors that account for differences in CVD among SSA and high-income countries include genetics contributions to disease occurrence. The genetic makeup and subsequent gene-environment and risk factor interactions may result in a differential magnitude of CVD and confer increased susceptibility in particular population groups (23).

Finally, social determinants of health are established as an indirect or proximate cause of CVD burden. Inadequate access to effective primary and secondary prevention measures due to inadequate funding results in ineffective screening, identification and management of CVDs and their risk factors (24).

Despite the presence of many studies measuring the prevalence of CVD risk factors in SSA, the absence of cardiovascular event data and pathological confirmation of CVD deaths creates a huge literature vacuum, thus the need for further studies to establish the much needed evidence. While the INDEPTH (International Network for the Demographic Evaluation of Populations and Their Health in low- and middle-income countries) network through WHO, has tried to fill this gap by using verbal autopsies to determine cause of death, much still remains to be done (7). The absence of clinical cardiovascular events data has led to the reliance on proxy makers, such as carotid intima-media thickness (CIMT), for CVD risk assessment and the identification of associated risk factors $(25,26)$.

The Human Heredity and Health Africa (H3A) consortium (https://h3africa. org) through the National Institute of Health (NIH) and Welcome Trust have funded seven studies of which the African-Wits-INDEPTH Genomic (AWIGen) study was one. The AWI-Gen study set out to identify the genomic and environmental risk factors for cardiometabolic diseases in SSA populations specifically Eastern, Western and Southern SSA regions $(27,28)$. This large panAfrican epidemiological and genetic study has collected socio-demographic, behavioral, anthropometric, metabolic, and genetic data on close to 12000 participants. The current thesis nested in the H3Africa AWI-Gen study used CIMT as a marker of sub-clinical atherosclerosis to investigate CVD risk in four SSA populations (Burkina Faso, Ghana, Kenya and South Africa). The participating countries and sites are shown in Figure 2. These countries are in different 
sub-regional blocks of SSA and are at different stages of the epidemiological transition and therefore present varying levels of CVD risk factors and environmental and genetic exposure. For comparison with other race-ethnic groups the AWI-Gen (27) and USE-IMT (29) individual participant data metaanalyses collaboration was formed as part of this thesis and results reported accordingly.

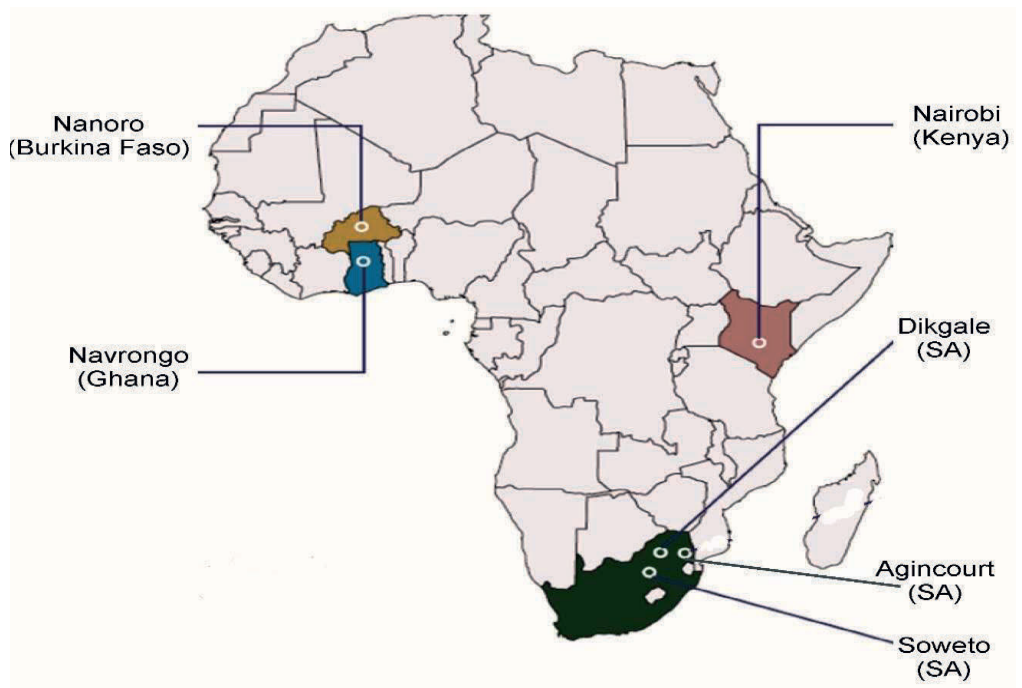

Figure 2: Map showing the six sites and four countries constituting the H3Africa AWI-Gen study; SA, South Africa

\section{Carotid intima-media thickness as a marker for Cardiovascular Disease}

A major precursor of CVD is atherosclerosis, a continuous process which often starts early in life, progresses with age and accelerates in the presence of risk factors (30). Several clinical and epidemiological studies have established CIMT measured using B-mode ultrasound scan as a marker for atherosclerosis and an alternative endpoint for identifying CVD risk factors $(25,26,30-32)$. CIMT (see Figure 3 ) is associated with progression of atherosclerosis and has proven to be useful in clinical trials for identifying anti-hypertensive and anti-lipid drugs that reduce progression of atherosclerosis $(25,26,32)$. This makes a strong case for the use of CIMT as a proxy for cardiovascular disease in settings where data on clinical events are absent. The strong association between CIMT and cardiovascular events and mortality has been reported in African Americans $(33,34)$. There is, however, a paucity of data from Africa on the link between CIMT and CVD and associated risk factors. The few studies conducted in Africa are mainly in subjects with established cardiometabolic diseases or in people living with HIV, hypertensive and diabetic patients and mostly have small sample sizes (35-42). The associated selection bias and small sample sizes make it difficult to 
apply findings to the general population. The link between CIMT and CVD risk in HIV and CVD free general population from SSA is scare in literature. In addition, a number of novel risk factors have been reported to be associated with CIMT and CVD, including microalbuminuria $(43,44)$. This is of interest in resource limited regions because of ease of measurement in urine samples.

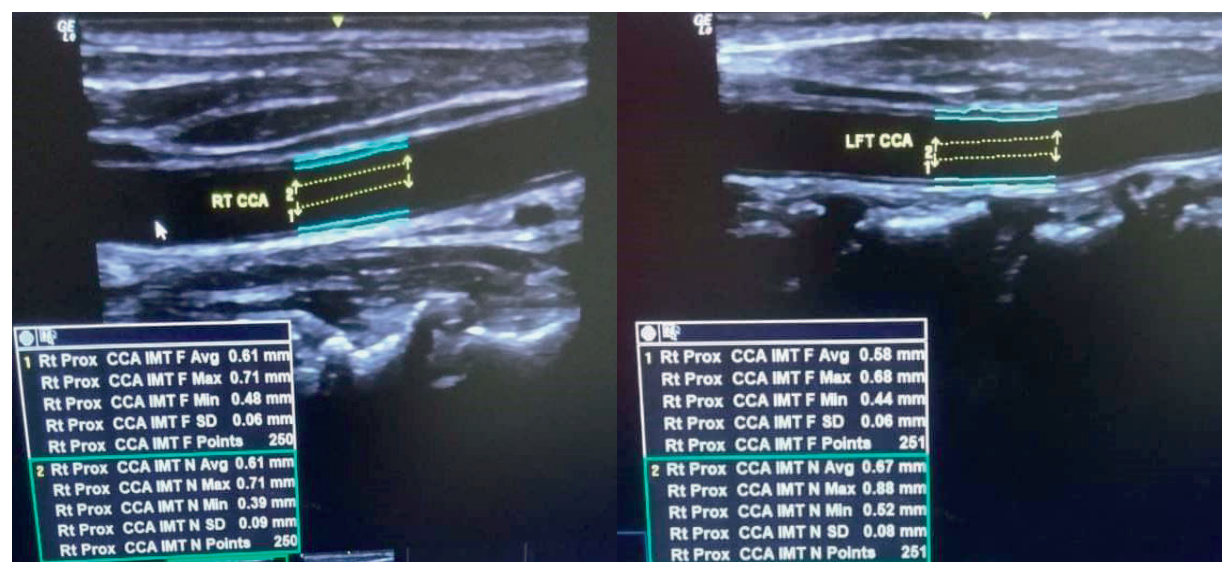

Figure 3: An image depicting common carotid intima media thickness showing automatic selection of the intima-media thickness interface (original image from the AWIGen study); the yellow lines represent the position of the cursor (10mm apart) to measure the carotid intima-media thickness (in blue lines); the displayed data give details on minimum, average and maximum intima-media thickness (IMT) of the common carotid artery.

\section{Thesis aims and objectives}

This thesis aimed to determine which CVD risk factors associate with CIMT in SSA populations, how these associations differ from those seen in other population groups and to assess the association of CIMT with cardiovascular risk.

This was approached through the following objectives:

I. Determining the association of classical CVD risk factors (age, sex, smoking, physical inactivity, poor diet, body mass index, blood pressure, hyperglycaemia and hypercholesterolemia) and HIV with CIMT (chapter 2).

II. Investigating the association of obesity phenotypes (body mass index, waist and hip circumference, visceral and subcutaneous adipose tissue) with CIMT in SSA (chapter 3). 
III. Describing race-ethnic differences in the association of classical CVD risk factors with CIMT in African, African American, Asian, European and Hispanic populations (chapter 4)

IV. Establishing the link between cardiovascular health using the American heart Association's (AHA) Cardiovascular Health Index (CVHI) and CIMT in SSA (chapter 5).

V. Investigating the relationship of CIMT and microalbuminuria with 10 -year predicted CVD risk using the 2018 Pooled Cohort Equation (chapter 6).

The results from this thesis would contribute knowledge to priority areas for the primary prevention of atherosclerotic cardiovascular disease.

\section{Thesis outline}

Following this chapter the thesis is sub-sectioned into 2 parts (see Figure 4). Part 1 contains 3 chapters (chapters 2 to 4 ). Chapter 2 looks at the association of the classical CVD risk factors with carotid intima thickness in Burkina Faso, Ghana, Kenya and South Africa. Due to the high level of obesity in certain regions of SSA, chapter 3 investigates the association of adiposity phenotypes with CIMT. Chapter 4 of the thesis explores the race-ethnic differences in the factors associated with CIMT in African, African American, Asian, European and Hispanic populations. Part 2 contains chapters 5 and 6. Chapter 5 describes the association of common screening tools (the cardiovascular health index often referred to as life's simple 7) with CIMT in SSA while Chapter 6 determines association of carotid atherosclerosis and microalbuminuria with predicted high 10 -year CVD risk in SSA. The thesis is concluded by discussing the similarities and differences in the risk factors associated with CIMT between African and other populations in Chapter 7. This chapter also examines the implications of our findings for policy on primary prevention, clinical care and further research (chapter 7). The thesis is completed by a summary of the key findings of this study in Chapter 8. 


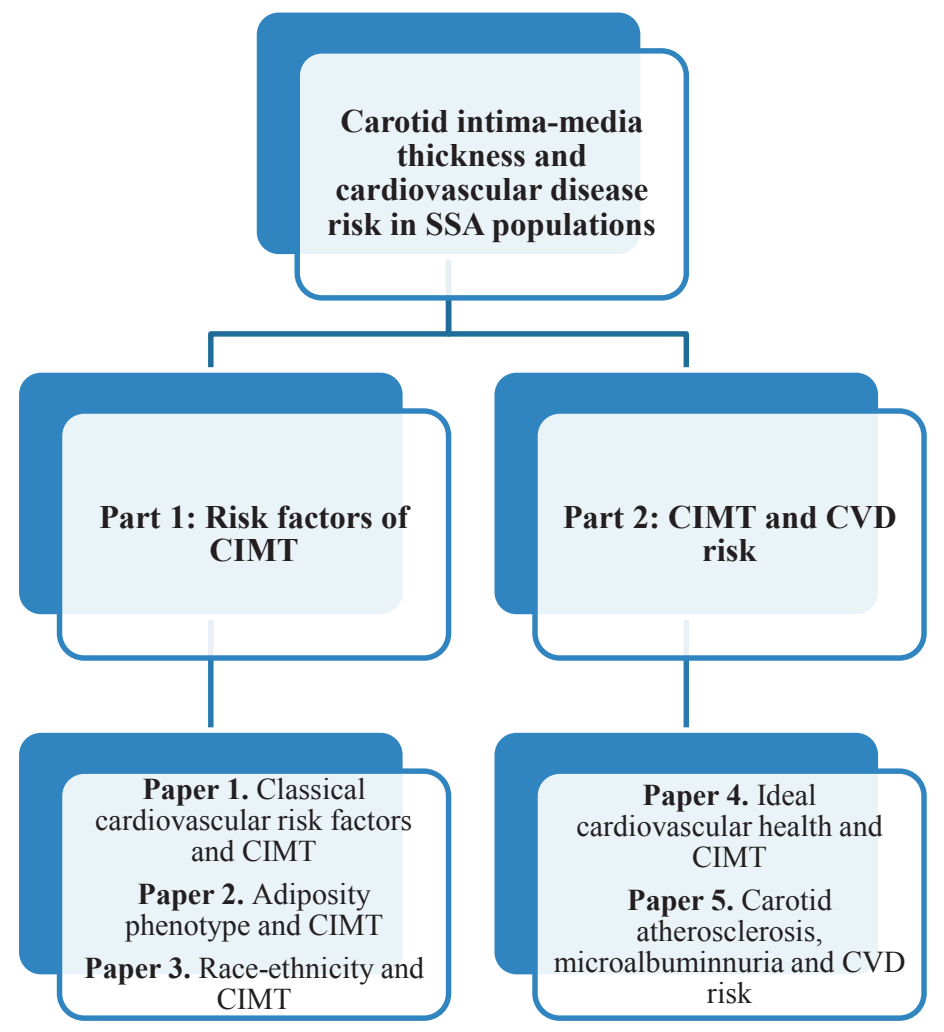

Figure 4: Overall thesis outline. Papers 1 to 3 represent chapters 2 to 4 of the thesis, whilst papers 4 and 5 are chapters 5 and 6. CVD, cardiovascular disease; CIMT, common carotid intima-media thickness and SSA, sub-Saharan African 


\section{References}

1. Roth GA, Forouzanfar MH, Moran AE, Barber R, Nguyen G, Feigin VL, et al. Demographic and epidemiologic drivers of global cardiovascular mortality. $\mathrm{N}$ Engl J Med. 2015;372(14):1333-41.

2. Vos $\mathrm{T}$, Lim SS, Abbafati C, Abbas KM, Abbasi M, Abbasifard M, et al. Global burden of 369 diseases and injuries in 204 countries and territories, 1990-2019: a systematic analysis for the Global Burden of Disease Study 2019. The Lancet. 2020;396(10258):1204-22.

3. Roth GA, Mensah GA, Johnson CO, Addolorato G, Ammirati E, Baddour LM, et al. Global Burden of Cardiovascular Diseases and Risk Factors, 1990-2019: Update From the GBD 2019 Study. J Am Coll Cardiol. 2020;76(25):2982-3021.

4. Hajar R. Framingham Contribution to Cardiovascular Disease. Heart Views. 2016;17(2):78-81.

5. Yadlowsky S, Hayward RA, Sussman JB, McClelland RL, Min YI, Basu S. Clinical Implications of Revised Pooled Cohort Equations for Estimating Atherosclerotic Cardiovascular Disease Risk. Ann Intern Med. 2018;169(1):20-9.

6. Murray CJL, Aravkin AY, Zheng P, Abbafati C, Abbas KM, Abbasi-Kangevari M, et al. Global burden of 87 risk factors in 204 countries and territories, 1990-2019: a systematic analysis for the Global Burden of Disease Study 2019. The Lancet. 2020;396(10258):1223-49.

7. Streatfield PK, Khan WA, Bhuiya A, Hanifi SM, Alam N, Bagagnan $\mathrm{CH}$, et al. Adult noncommunicable disease mortality in Africa and Asia: evidence from INDEPTH Health and Demographic Surveillance System sites. Glob Health Action. 2014;7:25365.

8. Yuyun MF, Sliwa K, Kengne AP, Mocumbi AO, Bukhman G. Cardiovascular Diseases in Sub-Saharan Africa Compared to High-Income Countries: An Epidemiological Perspective. Global Heart. 2020;15(1).

9. Ramsay M, Crowther NJ, Agongo G, Ali SA, Asiki G, Boua RP, et al. Regional and sexspecific variation in BMI distribution in four sub-Saharan African countries: The H3Africa AWI-Gen study. Glob Health Action. 2018;11(sup2):1556561.

10. Gomez-Olive FX, Ali SA, Made F, Kyobutungi C, Nonterah E, Micklesfield L, et al. Regional and Sex Differences in the Prevalence and Awareness of Hypertension: An H3Africa AWI-Gen Study Across 6 Sites in Sub-Saharan Africa. Glob Heart. 2017;12(2):81-90.

11. Nonterah EA, Debpuur C, Agongo G, Amenga-Etego L, Crowther NJ, Ramsay M, et al. Socio-demographic and behavioural determinants of body mass index among an adult population in rural Northern Ghana: the AWI-Gen study. Glob Health Action. 2018;11(sup2):1467588.

12. Akpa OM, Made F, Ojo A, Ovbiagele B, Adu D, Motala AA, et al. Regional Patterns and Association Between Obesity and Hypertension in Africa: Evidence From the H3Africa CHAIR Study. Hypertension. 2020:HYPERTENSIONAHA11914147. 
13. Bawah A, Houle B, Alam N, Razzaque A, Streatfield PK, Debpuur C, et al. The Evolving Demographic and Health Transition in Four Low- and Middle-Income Countries: Evidence from Four Sites in the INDEPTH Network of Longitudinal Health and Demographic Surveillance Systems. PLoS One. 2016;11(6):e0157281.

14. Ramsay M, Sankoh O, as members of the AWIGen study and the H3Africa Consortium. African partnerships through the H3Africa Consortium bring a genomic dimension to longitudinal population studies on the continent. Int J Epidemiol. 2016;45(2):305-8.

15. Wong EB, Olivier S, Gunda R, Koole O, Surujdeen A, Gareta D, et al. Convergence of infectious and non-communicable disease epidemics in rural South Africa: a cross-sectional, population-based multimorbidity study. The Lancet Global Health. 2021;9(7):e967-e76.

16. Basham CA, Smith SJ, Romanowski K, Johnston JC. Cardiovascular morbidity and mortality among persons diagnosed with tuberculosis: A systematic review and meta-analysis. PLoS One. 2020;15(7):e0235821.

17. So-Armah K, Benjamin LA, Bloomfield GS, Feinstein MJ, Hsue P, Njuguna B, et al. HIV and cardiovascular disease. The Lancet HIV. 2020;7(4):e279-e93.

18. Vos AG, Idris NS, Barth RE, Klipstein-Grobusch K, Grobbee DE. Pro-Inflammatory Markers in Relation to Cardiovascular Disease in HIV Infection. A Systematic Review. Plos One. 2016;11(1):e0147484.

19. Thivalapill N, Simelane T, Mthethwa N, Dlamini S, Lukhele B, Okello V, et al. Transition to dolutegravir is associated with an increase in the rate of $\mathrm{BMI}$ change in a cohort of virally suppressed adolescents. Clin Infect Dis. 2020.

20. Venter WDF, Sokhela S, Simmons B, Moorhouse M, Fairlie L, Mashabane N, et al. Dolutegravir with emtricitabine and tenofovir alafenamide or tenofovir disoproxil fumarate versus efavirenz, emtricitabine, and tenofovir disoproxil fumarate for initial treatment of HIV-1 infection (ADVANCE): week 96 results from a randomised, phase 3, non-inferiority trial. The Lancet HIV. 2020;7(10):e666-e76.

21. Manne-Goehler J, Montana L, Gomez-Olive FX, Rohr J, Harling G, Wagner RG, et al. The ART Advantage: Health Care Utilization for Diabetes and Hypertension in Rural South Africa. J Acquir Immune Defic Syndr. 2017;75(5):561-7.

22. Manne-Goehler J, Siedner MJ, Montana L, Harling G, Geldsetzer P, Rohr J, et al. Hypertension and diabetes control along the HIV care cascade in rural South Africa. J Int AIDS Soc. 2019;22(3):e25213.

23. Kathiresan S, Srivastava D. Genetics of human cardiovascular disease. Cell. 2012;148(6):1242-57.

24. Gaziano TA. Cardiovascular disease in the developing world and its cost-effective management. Circulation. 2005;112(23):3547-53.

25. Bots ML, Evans GW, Tegeler CH, Meijer R. Carotid Intima-media Thickness Measurements: Relations with Atherosclerosis, Risk of Cardiovascular Disease and Application in Randomized Controlled Trials. Chin Med J 2016;129(2):215-26.

26. Bots ML, Sutton-Tyrrell K. Lessons from the past and promises for the future for carotid intima-media thickness. J Am Coll Cardiol. 2012;60(17):1599-604. 
27. Ali SA, Soo C, Agongo G, Alberts M, Amenga-Etego L, Boua RP, et al. Genomic and environmental risk factors for cardiometabolic diseases in Africa: methods used for Phase 1 of the AWI-Gen population cross-sectional study. Glob Health Action. 2018;11(sup2):1507133.

28. Ramsay M, Crowther NJ, Tambo E, Agongo G, Baloyi V, Dikotope S, et al. H3Africa AWIGen Collaborative Centre: a resource to study the interplay between genomic and environmental risk factors for cardiometabolic diseases in four sub-Saharan African countries. Global Health, Epidemiology and Genomics. 2016;1(e20):1-13.

29. Den Ruijter HM, Peters SAE, Anderson TJ, Britton AR, Dekker JM, Eijkemans MJ, et al. Common Carotid Intima-Media Thickness Measurements in Cardiovascular Risk Prediction: A Meta-analysis. JAMA. 2012;308(8):796-803.

30. Libby P. Inflammation and Atherosclerosis. Circulation. 2002;105(9):1135-43.

31. Touboul PJ, Hennerici MG, Meairs S, Adams H, Amarenco P, Bornstein N, et al. Mannheim carotid intima-media thickness and plaque consensus (2004-2006-2011). An update on behalf of the advisory board of the 3rd, 4th and 5th watching the risk symposia, at the 13th, 15th and 20th European Stroke Conferences, Mannheim, Germany, 2004, Brussels, Belgium, 2006, and Hamburg, Germany, 2011. Cerebrovasc Dis. 2012;34(4):290-6.

32. Peters SAE, Grobbee DE, Bots ML. Carotid intima-media thickness: a suitable alternative for cardiovascular risk as outcome? European Journal of Cardiovascular Prevention \& Rehabilitation. 2011;18(2):167-74.

33. Villines TC, Hsu LL, Blackshear C, Nelson CR, Griswold M. Relation of Carotid IntimaMedia Thickness to Cardiovascular Events in Black Americans (From the Jackson Heart Study). Am J Cardiol. 2017;120(9):1528-32.

34. Gijsberts CM, Groenewegen KA, Hoefer IE, Eijkemans MJ, Asselbergs FW, Anderson $\mathrm{TJ}$, et al. Race/Ethnic Differences in the Associations of the Framingham Risk Factors with Carotid IMT and Cardiovascular Events. PLoS One. 2015;10(7):e0132321.

35. Schoffelen AF, de Groot E, Tempelman HA, Visseren FL, Hoepelman Al, Barth RE. Carotid Intima Media Thickness in Mainly Female HIV-Infected Subjects in Rural South Africa: Association With Cardiovascular but Not HIV-Related Factors. Clin Infect Dis. 2015;61(10):1606-14.

36. Ssinabulya I, Kayima J, Longenecker C, Luwedde M, Semitala F, Kambugu A, et al. Subclinical atherosclerosis among HIV-infected adults attending HIV/AIDS care at two large ambulatory HIV clinics in Uganda. PLoS One. 2014;9(2):e89537.

37. Feinstein MJ, Kim JH, Bibangambah P, Sentongo R, Martin JN, Tsai AC, et al. Ideal Cardiovascular Health and Carotid Atherosclerosis in a Mixed Cohort of HIV-Infected and Uninfected Ugandans. AIDS Res Hum Retroviruses. 2017;33(1):49-56.

38. Okeahialam BN, Alonge BA, Pam SD, Puepet FH. Carotid intima media thickness as a measure of cardiovascular disease burden in Nigerian Africans with hypertension and diabetes mellitus. Int J Vasc Med. 2011;2011:327171.

39. Napoli N, Zardi E, Strollo R, Arigliani M, Daverio A, Olearo F, et al. Increased carotid thickness in subjects with recently-diagnosed diabetes from rural Cameroon. PLoS One. 2012;7(8):e41316. 
40. Rheeder P, Duim-Beytell MC, Meijer R, Gustavsson T, Danler GKG, Ahmed RA, et al. Carotid intima-media thickness and its associations with type 2 diabetes mellitus in South Africans. Journal of Endocrinology, Metabolism and Diabetes of South Africa. 2014;17(3):135-40.

41. Siedner MJ, Bibangambah P, Kim JH, Lankowski A, Chang JL, Yang IT, et al. Treated HIV Infection and Progression of Carotid Atherosclerosis in Rural Uganda: A Prospective Observational Cohort Study. J Am Heart Assoc. 2021;10(12):e019994.

42. Vos AG, Barth RE, Klipstein-Grobusch K, Tempelman HA, Deville WLJ, Dodd C, et al. Cardiovascular Disease Burden in Rural Africa: Does HIV and Antiretroviral Treatment Play a Role?: Baseline Analysis of the Ndlovu Cohort Study. J Am Heart Assoc. 2020;9(7):e013466.

43. Gerstein HC, Mann JF, Yi Q, Zinman B, Dinneen SF, Hoogwerf B, et al. Albuminuria and risk of cardiovascular events, death, and heart failure in diabetic and nondiabetic individuals. . JAMA 2001;286: 421-6.

44. Libby P. Inflammation and Atherosclerosis. Circulation. 2002;105(9):1135-43. 
Subclinical atherosclerosis in African populations 


\section{Part 1:}

Risk factors for CIMT 


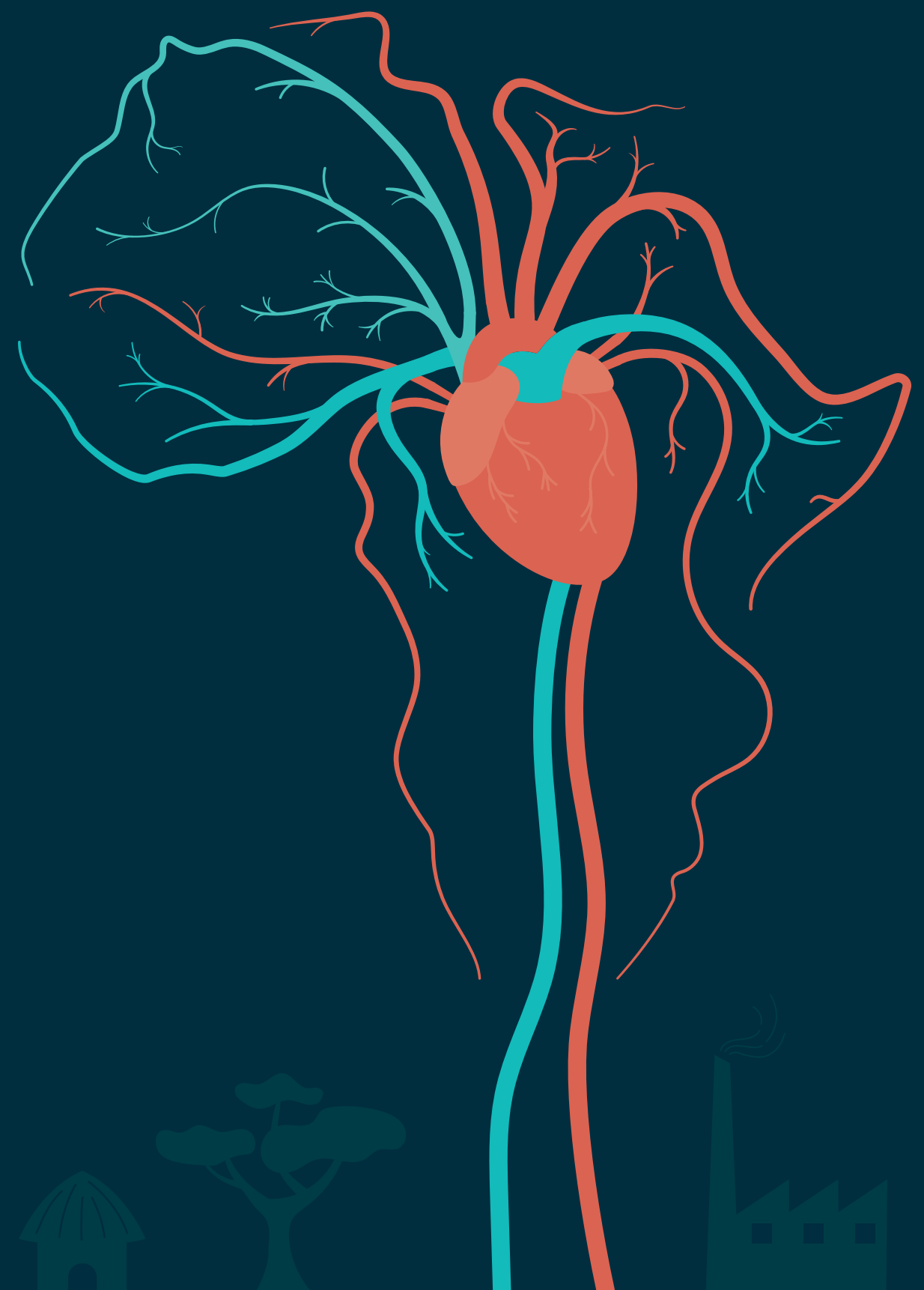




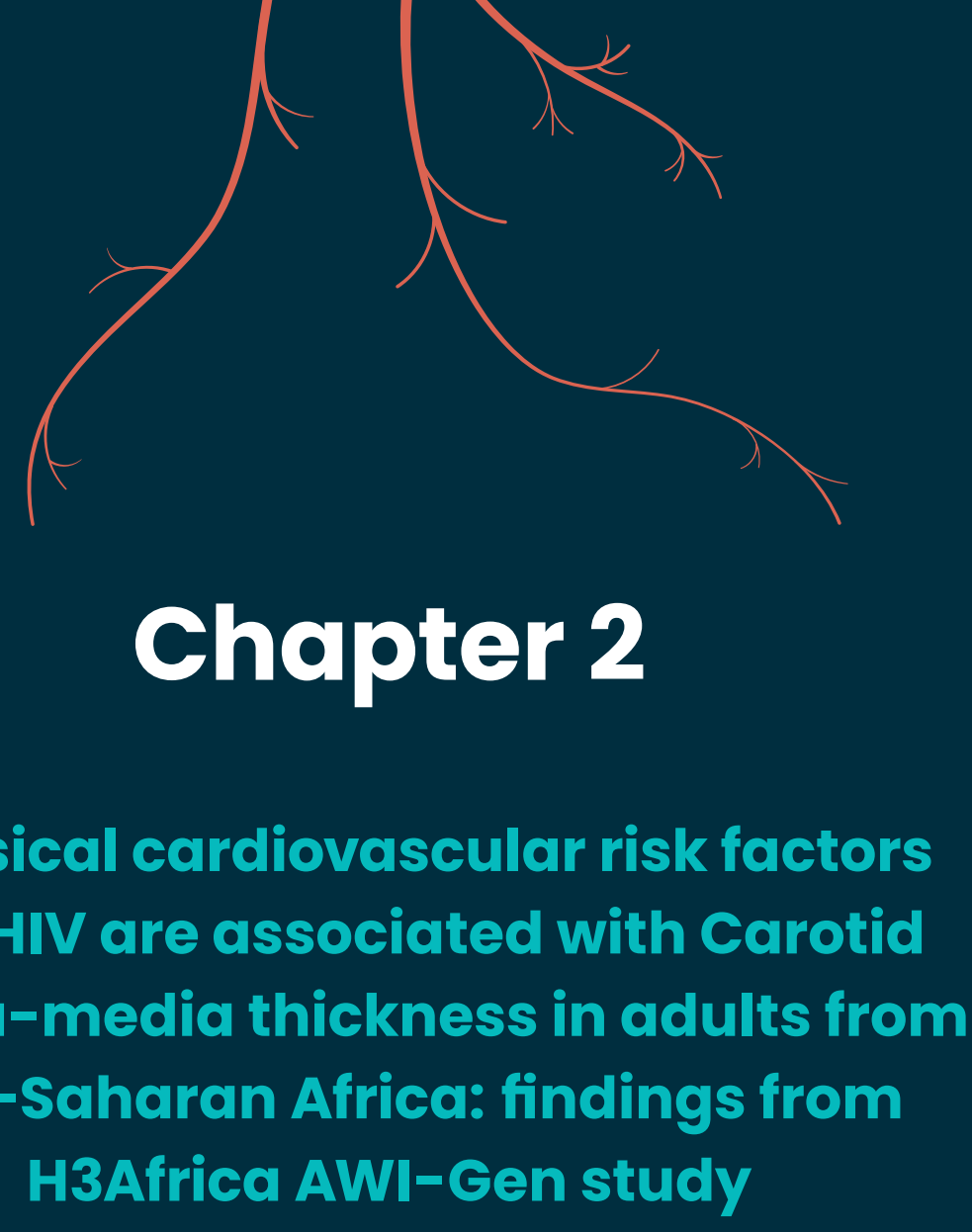

Nonterah EA, Boua PR, Klipstein-Grobusch K, Asiki G, Micklesfield LK, Agongo G, Ali SA, Mashinya F, Sorgho H, Nakanabo-Diallo S, Debpuur C, Kyobutungi C, Alberts M, Norris S, Tollman S, Tinto H, Soo CC, Mukomana F, Hazelhurst S, Wade AN, Kahn K, Oduro AR, Grobbee DE, Sankoh O, Ramsay M, Bots ML, Crowther NJ, as members and collaborators of AWI-Gen and the H3Africa Consortium* 


\section{Abstract}

Background: Studies on the determinants of carotid intima-media thickness (CIMT), a marker of sub-clinical atherosclerosis, mostly come from white, Asian, and diasporan black populations. We present CIMT data from sub-Saharan Africa, which is experiencing a rising burden of cardiovascular diseases and infectious diseases.

Methods and Results: The H3 (Human Hereditary and Health) in Africa's AWIGen (African-Wits-INDEPTH partnership for Genomic) study is a cross-sectional study conducted in adults aged 40 to 60 years from Burkina Faso, Kenya, Ghana, and South Africa. Cardiovascular disease risk and ultrasonography of the CIMT of right and left common carotids were measured. Multivariable linear and mixed-effect multilevel regression modeling was applied to determine factors related to CIMT. Data included 8872 adults ( $50.8 \%$ men), mean age of $50 \pm 6$ years with age- and sex-adjusted mean ( \pm SE) CIMT of $640 \pm 123 \mu \mathrm{m}$. Participants from Ghana and Burkina Faso had higher CIMT compared with other sites. Age $(\beta=6.77,95 \% \mathrm{Cl}[6.34,7.19])$, body mass index $(17.6[12.5,22.8])$, systolic blood pressure $(7.52[6.21,8.83])$, low-density lipoprotein cholesterol $(5.08[2.10,8.06])$ and men $(10.3[4.75,15.9])$ were associated with higher CIMT. Smoking was associated with higher CIMT in men. High-density lipoprotein cholesterol (-12.2 $[-17.9,-6.41])$, alcohol consumption $(-13.5[-19.1,-7.91])$ and HIV $(-8.86[-15.7,-2.03])$ were inversely associated with CIMT.

Conclusions: Given the rising prevalence of cardiovascular diseases risk factors in sub-Saharan Africa, atherosclerotic diseases may become a major pan-African epidemic unless preventive measures are taken particularly for prevention of hypertension, obesity, and smoking. HIV-specific studies are needed to fully understand the association between HIV and CIMT in subSaharan Africa.

Key Words: cardiovascular disease; carotid intima-media thickness; epidemiological transition; prevention and sub-Saharan Africa 


\section{Clinical Perspective}

\section{What Is New?}

- This the first study involving indigenous African populations drawn from 4 countries at different stages of epidemiological transition, to demonstrate that classical cardiovascular risk factors such as age, male sex, systolic blood pressure, serum cholesterol, and obesity are major drivers of increased carotid intima-media thickness.

- HIV infection was not associated with higher carotid intima-media thickness levels.

\section{What Are the Clinical Implications?}

- Interventions focused on classical, modifiable cardiovascular disease risk factors will attenuate atherosclerotic risk in sub-Saharan African populations.

- HIV infection may not be a risk factor for atherosclerotic diseases in subSaharan African.

- Results from recent studies in sub-Saharan African have reported a lower prevalence of cardiovascular disease risk factors in subjects living with HIV and this new paradigm suggests that the HIV care cascade may be an effective resource for the prevention and control of cardiovascular diseases among people living with HIV. 


\section{Introduction}

Global morbidity and mortality attributable to noncommunicable diseases are increasing with cardiovascular diseases (CVD) being a significant contributor (1). A greater proportion of the annual CVD-related deaths occur between 30 to 70 years of age and $85 \%$ of these deaths occur in low- and middle-income countries, and are projected to increase further (2). A major contributor to the burden of cardiovascular morbidity and mortality is the development of atherosclerosis, a continuous process which often starts early in life and progresses with age (3).

Exposure to unfavorable levels of established cardiovascular risk factors such as high systolic blood pressure, smoking, and dyslipidemia leads to the accelerated development of atherosclerosis. The measurement of sub-clinical atherosclerosis by the assessment of carotid intima-media thickness (CIMT) may provide information about the cardiovascular status of a population (4-7). However, epidemiological data on the prevalence and determinants of high CIMT arise largely from studies in whites, Asians, and Africans in the diaspora or blacks, which may not reflect the situation among Africans living in subSaharan Africa (SSA) $(4,8,9)$. Africa is currently engulfed in a wave of complex epidemiological transition that is characterized by extensive urbanization with concomitant lifestyle changes such as consumption of calorie dense diets and a decrease in physical activity $(10,11)$. The surge in CVD risk factors, such as obesity and hypertension, adds to the complex milieu of a high burden of infectious diseases such as HIV, tuberculosis, and malaria (12).

Despite this double burden of non-communicable and infectious diseases in Africa, large cohort studies with harmonized data on CVDs are lacking (10). To formulate appropriate interventions to decrease CIMT as a proxy for subclinical atherosclerosis, it is important to identify the specific risk factors associated with CIMT in SSA populations.

Therefore, the aim of the study was to measure CIMT and identify risk factors associated with CIMT in a large SSA population. This was achieved by measuring CIMT levels within the AWI-Gen (African-Wits-INDEPTH [International Network for the Demographic Evaluation of Populations and their Health in low- and middleincome countries] Partnership for Genomic studies). This is a large pan-African epidemiological and genetic study that has collected sociodemographic, behavioral, anthropometric, metabolic, and genetic data on close to 12000 participants from 4 countries within SSA $(13,14)$. 


\section{Materials and Methods}

All data and materials for the AWI-Gen study that support the findings in this paper will be made available in the European Genome-phenome Archive under the set of projects related to the Human Heredity and Health in Africa (H3Africa) Consortium. Details about access to data can be found in the document titled "H3Africa Data and Biospecimen Access Committee Guidelines," available in the consortium documents section of the H3Africa website (www.h3africa.org).

\section{Study Design and Study Population}

We conducted a population-based cross-sectional study as part of the AWIGen partnership, a National Institutes of Health-funded Collaborative Centre of the Human Heredity and Health in Africa (H3Africa) Consortium. Six study sites in 4 sub-Saharan African (SSA) countries were involved in the AWI-Gen study (Figure 1). Three of the sites were in South Africa of which two are rural and one is urban. The rural sites were the Dikgale health and demographic surveillance site (HDSS) (15) affiliated with the Department of Pathology and Medical Science, University of Limpopo, and the Agincourt Health and Demographic Surveillance System Site (HDSS) (16) managed by the Medical Research Council/ Wits Rural Public Health and Health Transitions Research Unit, University of the Witwatersrand. The urban South African site is the MRC/Wits Developmental Pathways for Health Research Unit, University of the Witwatersrand, in Soweto (17). There was 1 urban site in Nairobi, Kenya: the African Population and Health Research Center HDSS (18). Finally, there were 2 rural sites in West Africa: the Navrongo HDSS hosted by the Navrongo Health Research Center in Ghana (19), and the Nanoro HDSS hosted by the Institut de Recherché en Sciences de la Santé Clinical Research Unit of Nanoro in Burkina Faso (20). 


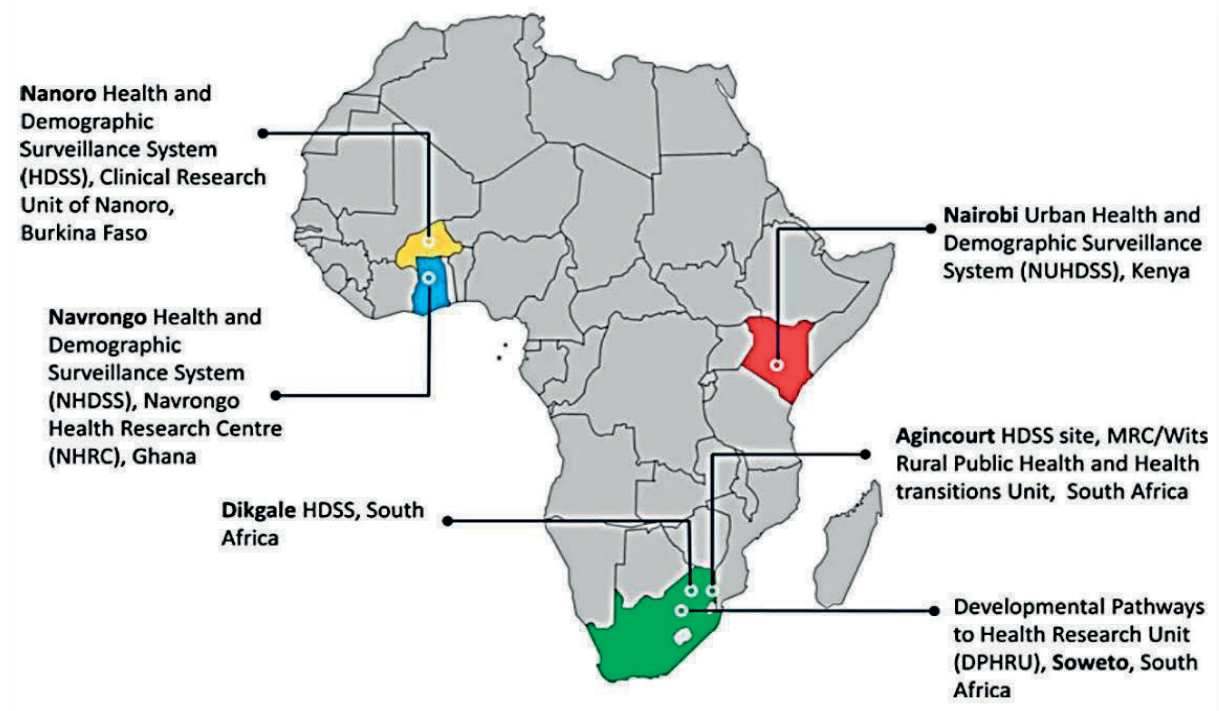

Figure 1. The sites constituting the H3Africa AWI-Gen study. Reprinted from Ramsay et al (13) with permission. Copyright (02016, Cambridge University Press

Included in the study were adults aged 40 to 60 years resident in the various sites. Exclusion criteria were current pregnancy and inability to complete the prescribed study procedures. Similar numbers of women and men were randomly sampled from each of the sites using existing sampling frames for the respective HDSS sites $(13,14)$. Only men from Soweto were included in these analyses as women contributing to the study population in Soweto, were the caregivers of the Birth to $20+$ Cohort $^{17}$ and did not have CIMT measurements. Our study population covers 3 of the 5 sub-continental blocks of Africa and therefore represents a large proportion of the geographical (covering both rural and urban areas) and social variability of the SSA region.

\section{Ethical Considerations}

The AWI-Gen study received overall ethical approval from the Human Research Ethics Committee of the University of the Witwatersrand, Johannesburg, South Africa (approval identification numbers: M121029; M170880), as well as from the appropriate ethics committees covering the Dikgale, Navrongo, Nanoro, and Nairobi sites. Community engagement activities were completed at each site to introduce the study to community leaders before commencement of the field work. Written and signed or thumb-printed informed consent was obtained from each participant before performing prescribed study procedures. In compliance with good clinical practice, participants who were found to have clinically overt CVDs were linked to health care by issuing referral letters in accordance with the healthcare system in the particular study site. 


\section{Data Collection}

Details of data collection methods and procedures have been described elsewhere $(13,14,21)$. Briefly, a paper-based questionnaire was used to collect information from 5 of the sites while a Computer-Assisted Personalized Interview was used to collect information from the Agincourt site in South Africa. Information collected included demography, family ethnicity, education, household attributes, substance use (tobacco, alcohol, and drugs), infectious disease history (HIV, TB, and malaria), history of cardiovascular and metabolic diseases (diabetes mellitus, stroke, hypertension, angina, heart attack, congestive heart failure, obesity, and high cholesterol), thyroid disease, kidney disease, and physical activity. Data were then entered into the REDCap (Research Electronic Data Capture) system hosted at the University of the Witwatersrand, Johannesburg $(22,23)$. Data entry quality control to identify outliers, duplicate information, and missing data were completed on $10 \%$ of the data per site.

\section{Carotid Ultrasonography}

\section{Training}

To maintain uniformity across the sites, carotid ultrasonography procedures were standardized, and technicians trained centrally by a certified sonographer at the MRC/Wits Developmental Pathways for Health Research Unit at the Chris Hani Baragwanath Hospital, Soweto, South Africa. The identified technicians from the study sites were clinicians, nurses, or biomedical scientists. To ensure reproducibility and reduce CIMT measurement variability, masked repeated measurements of 15 volunteers were conducted by each trainee and the lead trainer. The coefficient of variation between and within trainees was calculated and maintained at $<2 \%$. Subsequently, the same settings and calibrations of the ultrasound equipment (linear-array 12L-RS transducer with a B-mode LOGIQ e ultrasound machine, GE Healthcare, CT, USA) were used for data collection at all sites throughout the entire recruitment period.

\section{Image acquisition}

To measure the right carotid, the participant was asked to lie down in a supine position with a pillow underneath the neck for slight extension, head turned towards the left at a $45^{\circ}$ angle and gel applied to the exposed neck area. Using the 2 sternocleidomastoid muscles as landmarks, the exposed area was scanned along the longitudinal plane until the common carotid artery (CCA) was found, and an image frozen. The operator then identified a continuous $1-\mathrm{cm}$ segment $(10 \mathrm{~mm})$ of the CCA far wall. The operator then placed a cursor between 2 points ( $10 \mathrm{~mm}$ apart) on this identified segment of the far wall with the proximal starting point $1 \mathrm{~cm}$ from the bulb of the CCA. The ultrasound machine software then automatically detected the intima-lumen and the 
media adventitia interfaces and calculated the minimum, maximum, and mean common CIMT in millimeters and to 2 decimal places. To measure the left carotid, the participant's head was turned to the opposite side, and the process was repeated. This approach was selected over measuring "multiple carotid segments" because it was easier to measure and more reproducible enabling its widespread use at all study sites. A recent study demonstrates that measuring CCA IMT is a good alternative compared with multiple segments in terms of prediction of risk of CVD events (24). Additional quality control (QC) before analyses included the exclusion of CCA IMT $>1.5 \mathrm{~mm}$ as this is indicative of plaque. Images with $>50 \%$ differences between minimum and maximum CCA IMT were also excluded from the analytical data set. ${ }^{5}$ The far walls of both the left and right common carotid artery were averaged to determine mean CIMT thickness in millimeters as the main outcome variable. This was then converted to micrometers for the regression analyses.

\section{Assessment of Sociodemographic, Behavioral, Anthropometric, Blood Pressure, Biochemical and HIV-Related Variables}

Age at the time of data collection and sex of participants were self-reported. Highest level of education attained was self-reported and categorized as no formal education, completion of primary, secondary or tertiary education. Household socioeconomic status (SES) was assessed using the INDEPTH health equity tool which is an asset index generated by using principal component analysis to combine data on household possessions (http://indepth-network. org/resources/indepth-health-equity-tool-measuring-socio-economicstatus). The asset score generated was categorized into quintiles (Ql=poorest, Q2=poorer, Q3=poor, Q4=less poor and Q5=least poor) and this was computed separately for each of the sites. Smoking status was assessed by asking subjects if they had ever smoked any tobacco products such as cigarettes, cigars or pipes, and if they were current or past users of such products, Smoking was then categorized as "never," "current," and "past." For the purpose of these analyses current and past were combined as "smokers." Self-reported alcohol use was assessed using the 4-item CAGE (cut-annoyed-guilty-eye) questionnaire ${ }^{25}$ and subsequently categorized as current or never/previous use. The Global Physical Activity Questionnaire was used to compute moderate-to-vigorous intensity physical activity (MVPA) in minutes per week. Participants were said to be active if their MVPA was $\geq 150 \mathrm{~min} /$ week or inactive if their MVPA was $<150 \mathrm{~min} /$ week (26).

Standing height to the nearest $0.1 \mathrm{~cm}$ and weight to the nearest $0.1 \mathrm{~kg}$ were measured without shoes and in light clothes using a Harpenden stadiometer (Holtain, Crymych, Wales) fixed to the wall and a digital calibrated weighing scale, respectively. Resting systolic blood pressure (SBP) and diastolic blood pressure were measured using a digital sphygmomanometer (Omron M6, 
Omron, Kyoto, Japan) with the participant seated with their arm at the level of the chest and with an appropriate-sized cuff. Three readings were taken at 2-minute intervals with the first reading discarded and the average of the final 2 readings taken as the current blood pressure reading.

Overnight fasting serum lipids and glucose were measured using an automated chemistry analyzer (Randox RX Daytona+, Crumlin, Northern Ireland). All serum samples were analyzed at the University of the Witwatersrand Developmental Pathways for Health Research Unit laboratory, Chris Hani Baragwanath Academic Hospital, Soweto, South Africa. The low density lipoprotein cholesterol (LDL-C) was calculated using the Friedewald equation (27).

Self-reported HIV status was determined at all sites and in addition HIV testing (using locally-available rapid-test kits) was offered to all participants from Kenya and South Africa. In South Africa all known HIV-positive participants were asked whether they were receiving antiretroviral therapy (ART). In Agincourt, HIV status was determined by use of Vironostika Uniform 11 [Biomeriuex, France] screening assay as part of the HAALSI (Health and Ageing in Africa: a Longitudinal Study of an INDEPTH Community in South Africa) study (28), several months before performing measurements on a subset of these participants for the AWI-Gen study (14). The HIV status could therefore have changed at the time of recruitment into the AWI-Gen study. The prevalence of HIV infection at the Burkina Faso and Ghana sites is known to be between $1 \%$ to $2 \%(29,30)$.

\section{Statistical Analysis}

Continuous data are presented as means \pm SD while categorical data are presented as proportions. Age- and sex-adjusted mean (with standard error of the mean) CIMT was computed and presented for the various sites stratified by sex. One-way analysis of variance was used to determine the differences in mean CIMT levels between the sites whilst the Student t-test was used to determine differences between women and men within the sites. We used various multivariable linear regression models to determine the factors associated with CIMT. In model 1 we adjusted for the classical CVD risk factors. These factors are often used in risk prediction equations (31) to determine the risk of dying from atherosclerotic cardiovascular diseases and include age, sex, current smoking, SBP, fasting glucose, HDL-C, and LDL-C. We replaced total cholesterol with LDL-C because of its key role in the pathogenesis of atherosclerosis and treatment monitoring $(3,32)$. In model 2 , we adjusted for body mass index (BMI), physical activity and alcohol consumption in addition to factors from model 1. In model 3, since household SES and education are a proxy for urbanization and social determinants of health $(33,34)$, we adjusted for these in addition to factors from model 2. Finally, considering the high burden 
of HIV infection in SSA, we adjusted for HIV status in model 4 in addition to the variables included in model 3 . We also adjusted for the effect of site in all 4 of the multiple linear regression models. The final model (model 4) therefore included age, sex, smoking, SBP, fasting glucose, $\mathrm{HDL}-\mathrm{C}$, LDL-C, BMI, alcohol consumption, physical activity, education status, household SES, HIV status and site.

We checked this model for multi-collinearity using the variance inflation factor ensuring that the variance inflation factor for each variable was $<10$. We added multiplicative interaction terms to this model to evaluate whether relations between risk factors and common CIMT differed by sex and between sites. If interaction terms were statistically significant $(P<0.05)$, findings were presented for separate strata (men and women; across sites). In a further sub-analysis involving only the South African sites and Kenya we adjusted for objectively measured HIV status and for the South African sites only we adjusted for the effect of ART. This was because only at these sites was HIV status measured objectively and ART status was assessed only in the South African sites.

To fully account for within site differences, we further conducted mixed-effect multi-level $(\mathrm{ML})$ regression using site as a random effect in the final model (model 4 ) of the multiple linear regression analysis. We also checked for the role of interaction of HIV with age, sex, and household SES in the mixed-effect ML model. A likelihood ratio test was then used to compare the best fitting model between the single multivariable linear regression model, the mixed-effect $M L$ regression model and the mixed-effect $M L$ regression model that included interaction terms. Results are reported as unstandardised $\beta$-coefficients with corresponding $95 \% \mathrm{Cls}$. Statistical significance was set at a 2 -sided $\mathrm{P}<0.05$. For educational status and household SES, which had >2 categories, a post estimation test was used to obtain a single $P$ value for the estimate in the models. All data were analyzed using STATA 14.2 (College Station, TX 77845, USA) software.

\section{Results}

Data were available for 10363 participants drawn from all 6 sites. However, 1491 of these were excluded from the data analysis because they either had no CIMT data or did not meet quality control criteria. The final analytical data set therefore consisted of 8872 participants.

\section{Sociodemographic Characteristics}

The mean ( \pm SD) age of the total study population was $49.9 \pm 5.83$ years and men comprised $50.8 \%$ of study participants (Table 1). Nairobi, Kenya had the youngest ( $48.5 \pm 5.43$ years) while Navrongo, Ghana had the oldest (51.1 \pm 5.75 
years) population. The two West African sites, Nanoro in Burkina Faso (83.0\%) and Navrongo in Ghana (70.5\%), had the highest number of participants with no formal education, while all three South African sites (8.45\% Agincourt, $3.56 \%$ Dikgale and $14.4 \%$ among men in Soweto) and Nairobi (3.69\%) had the highest number of participants with tertiary education. More than two-thirds of participants in all countries were in the 2 highest quintiles (less and least poor) of household socio-economic status (Table 1). 


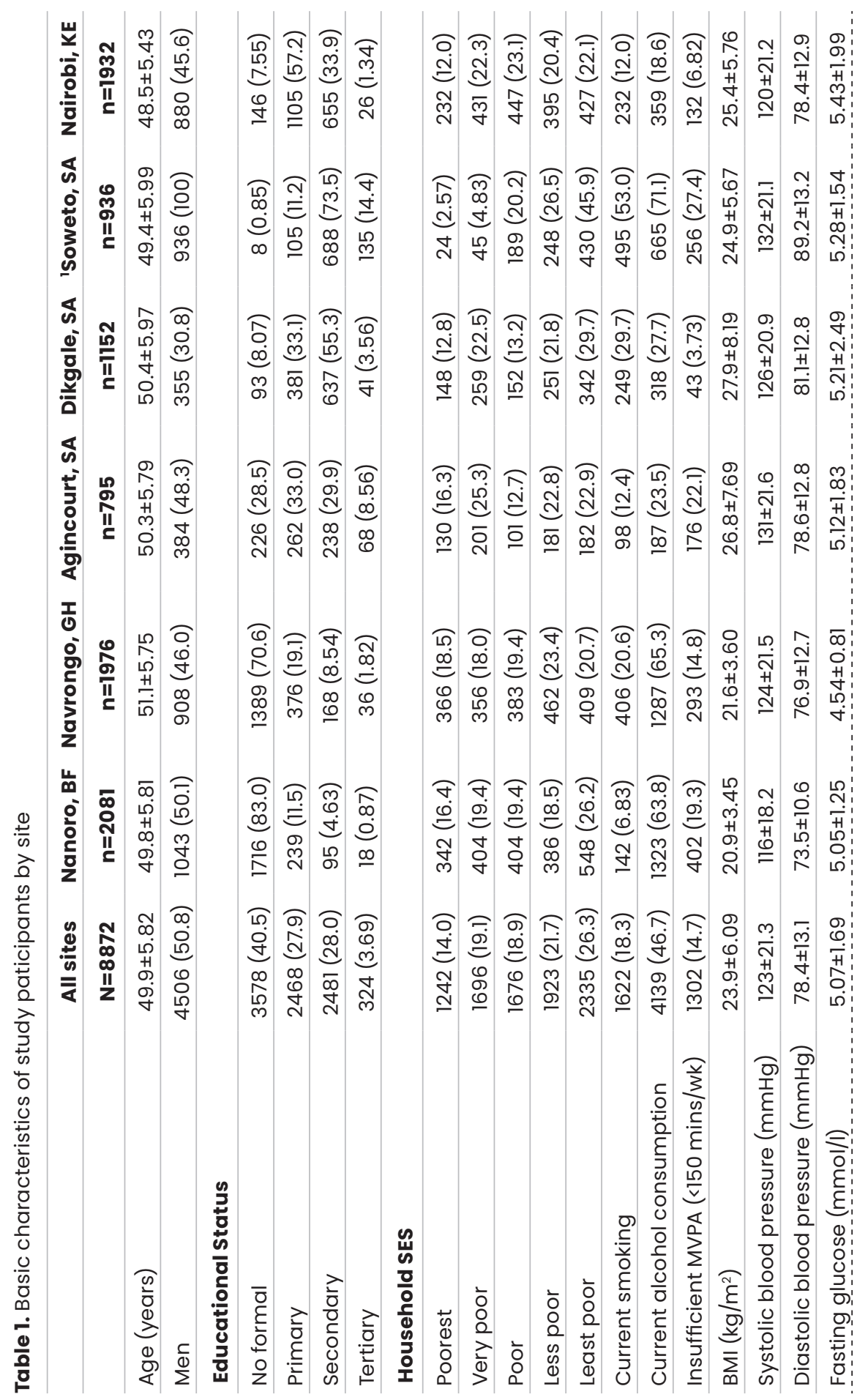




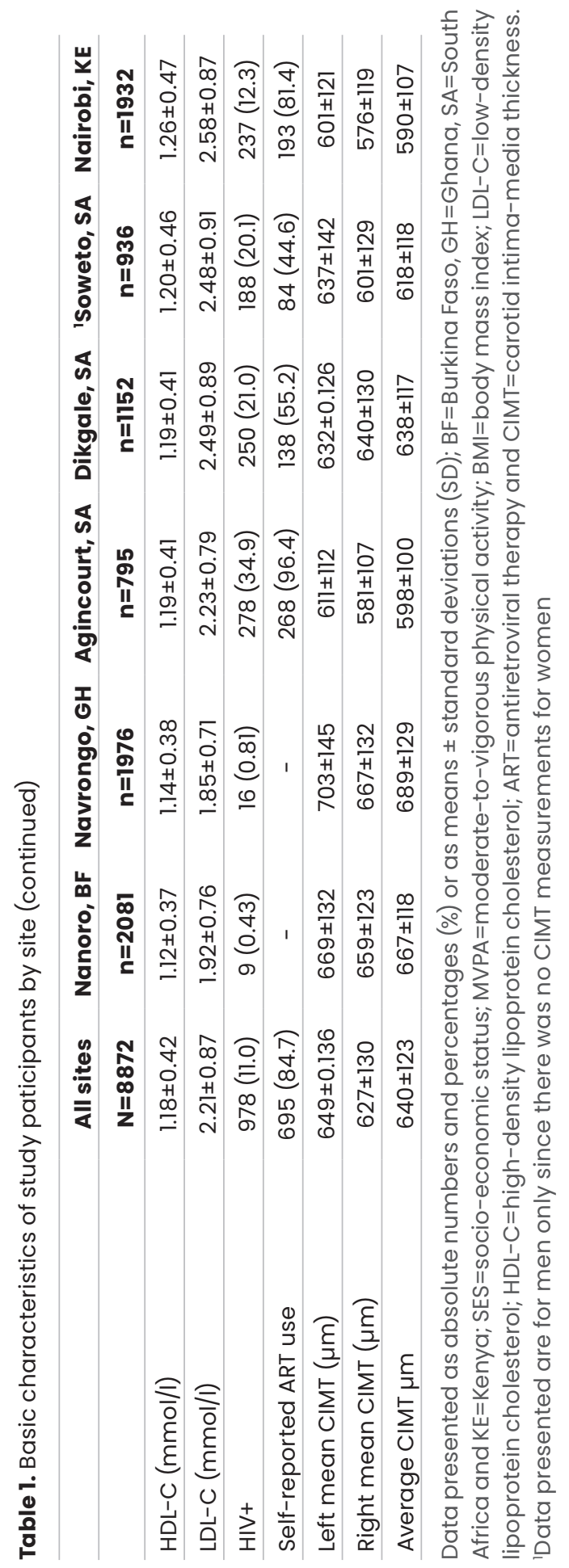




\section{Behavioral Factors}

The prevalence of smoking was higher among men in Soweto (53\%) and Dikgale (29.7\%) in South Africa and Navrongo, Ghana (20.6\%) compared with Agincourt, South Africa (12.4\%), Nairobi, Kenya (12.0\%) and Nanoro, Burkina Faso (6.83\%). Men in Soweto (71.1\%) and the 2 West African sites (65.3\% in Ghana and $63.8 \%$ in Burkina Faso) recorded higher current alcohol consumption than Dikgale (27.7\%), Agincourt (23.5\%), and Nairobi (18.6\%). The total population was fairly active with more than two-thirds of adults across all countries meeting physical recommendations of $\geq 150$ minutes of moderate-to-vigorous intensity physical activity per week (see Table 1).

\section{Anthropometric, Blood Pressure, Biochemical Variables, and HIV Infection}

The average BMI was $23.9 \pm 6.09 \mathrm{~kg} / \mathrm{m} 2$, with the rural sites of Burkina Faso $(20.9 \pm 3.45 \mathrm{~kg} / \mathrm{m} 2)$ and Ghana $(21.6 \pm 3.6 \mathrm{~kg} / \mathrm{m} 2)$ in West Africa recording lower BMls than the urban sites in Kenya $(25.4 \pm 5.76 \mathrm{~kg} / \mathrm{m} 2)$ and the urban $(25.0 \pm 5.67$ $\mathrm{kg} / \mathrm{m} 2$ in Soweto) and rural $(26.8 \pm 7.69 \mathrm{~kg} / \mathrm{m} 2$ in Agincourt and $27.9 \pm 8.19 \mathrm{~kg} / \mathrm{m} 2$ in Dikgale) sites in South Africa (Table 1). The average SBP and diastolic blood pressure were highest in all 3 South African sites and Kenya compared with the West African sites. Fasting blood glucose was highest in Kenya (5.43 1.99 $\mathrm{mmol} / \mathrm{L})$, followed by the South African sites $(5.12 \pm 1.83 \mathrm{mmol} / \mathrm{L}$ in Agincourt, $5.21 \pm 2.49 \mathrm{mmol} / \mathrm{L}$ in Dikgale and $5.28 \pm 1.54 \mathrm{mmol} / \mathrm{L}$ in Soweto), Burkina Faso $(5.05 \pm 1.25 \mathrm{mmol} / \mathrm{L})$ and Navrongo $(4.54 \pm 0.81 \mathrm{mmol} / \mathrm{L})$. A similar pattern was observed for HDL-C and LDL-C. The prevalence of HIV was highest in South Africa (34.9\% in Agincourt, $21 \%$ in Dikgale and $20 \%$ in Soweto) followed by Kenya (12.3\%) and $<1 \%$ in Burkina Faso and Ghana (Table 1). Self-reported use of ART among HIV positive participants was lowest at Soweto (44.6\%) followed by Dikgale with $55.2 \%$ and as high as $81.4 \%$ in Nairobi and $96.4 \%$ in Agincourt.

\section{CIMT Measurements}

Mean CIMT of the left CCA was higher than the right CCA (Table 1) in all 6 sites $(P<0.001$ in all sites). Age and sex adjusted mean ( \pm standard errors) levels of the average CIMT for each sample at the different sites are presented in Figure 1. The average CIMT for the entire population was $640 \pm 113 \mu \mathrm{m}$. Mean CIMT was significantly different between all sites $(P<0.001)$ with Ghana $(689 \pm 129 \mu \mathrm{m})$ and Burkina Faso $(667 \pm 118 \mu \mathrm{m})$ recording higher common CIMT levels than the South African sites (598 $\pm 100 \mu \mathrm{m}$ in Agincourt, 638 $\pm 117 \mathrm{Im}$ in Dikgale and 0.64 \pm 0.002 $\mu \mathrm{m}$ in Soweto) and Kenya $(590 \pm 107 \mu \mathrm{m})$. Women in Kenya had higher CIMT compared with men $(\mathrm{P}=0.022)$ while the reverse was observed in Burkina Faso $(P<0.001$ ) (Figure 2). In Ghana $(P=0.657$ ) and South Africa (Agincourt, $P=0.501$ and Dikgale, $\mathrm{P}=0.935)$, women and men had similar levels of common CIMT. 


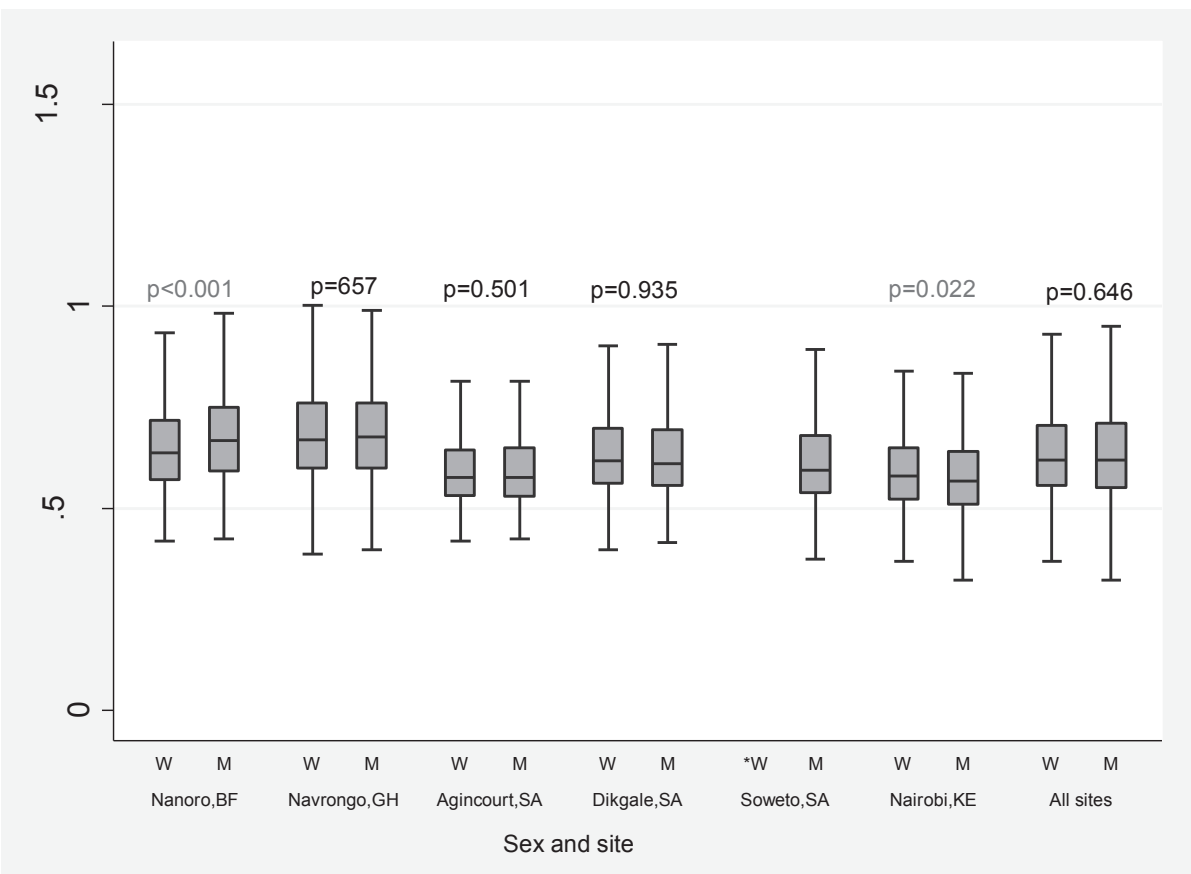

Figure 2: Distribution of mean levels of common CIMT (in $\mathrm{mm}$ ) across 4 SSA countries stratified by sex. Differences between women (W) and men (M) were generated using sample t-test with equal variance; *Data not available for women in Soweto. BF, Burkina Faso; GH, Ghana; KE, Kenya; SA, South Africa.

\section{Factors Associated With Common CIMT}

The factors associated with CIMT in the combined population within univariate analyses are shown in Table Sl. Adjusted regression models were then built sequentially and the factors associated with CIMT in the various models are displayed in Table 2. These analyses show that of the variables included in model 1, age, SBP, HDL-C and LDL-C remained significant through to model 4 , whilst the effect of sex became significant from model 2 onwards. Smoking status was not significantly associated with CIMT in any of the models but its effect did strengthen across the models, whilst the effect of glucose weakened. Among the variables added in model 2, BMl and alcohol use both were significant and remained so, whilst MVPA was not significantly associated with CIMT in any of the models. Educational status and household SES included in model 3 were not significantly associated with CIMT, whilst HIV status, which was added in model 4 , was significant. Model 1 explained $23.4 \%$ of the variance in CIMT whilst model 4 explained $24.4 \%$ of the variance. To further understand the effect of HIV infection on CIMT, we conducted a sensitivity analysis, where we included objectively assessed HIV status from the South African sites and Kenya only. In 
this model we observed an even stronger inverse association with CIMT (-15.3 $[-24.2,-6.31])$. We conducted a further sub-analysis on HIV participants who used ART and observed that ART was associated with lower CIMT levels (-70.1 [-165, -25.6] in Agincourt; -5.05 [-9.52, -1.29] in Dikgale and -48.6 [-82.9, -14.2] in Soweto). This model included only participants from South Africa, which was the only country in which ART use was assessed.

To determine the within site variation, we conducted a mixed-effect $\mathrm{ML}$ regression analyses. Presented in Table 3 is a comparison of multivariable linear (model 4) and mixed-effect ML regression analyses (model 5) and mixed-effect $M L$ regression analyses including interaction terms (model 6). The likelihood ratio test ( $L R \times 2=498.02 ; P<0.001)$ indicated that the mixed-effect $M L$ model (model 5) had a better fit than the multivariable linear regression model from Table 2 (model 4). In model 5, the association between classical cardiovascular risk factors and CIMT were similar to the multivariable linear regression model. However, HIV, although showing a similar direction of effect $(-5.81[-13.82,2.21])$, the observed effect was no longer significant $(P=0.156)$. Further to this, physical activity was associated with lower CIMT levels (-8.17 [-14.81, -1.53]; $P=0.016)$. When interaction terms were introduced into this model (model 6), we noted that HIV did not interact with age $(P=0.306)$, sex $(P=0.252)$ or household SES $(P=0.877)$. The likelihood ratio test ( $\operatorname{LR} \chi 2=47.88 ; P=0.318)$, showed a better fit for model 5 than for model 6. 


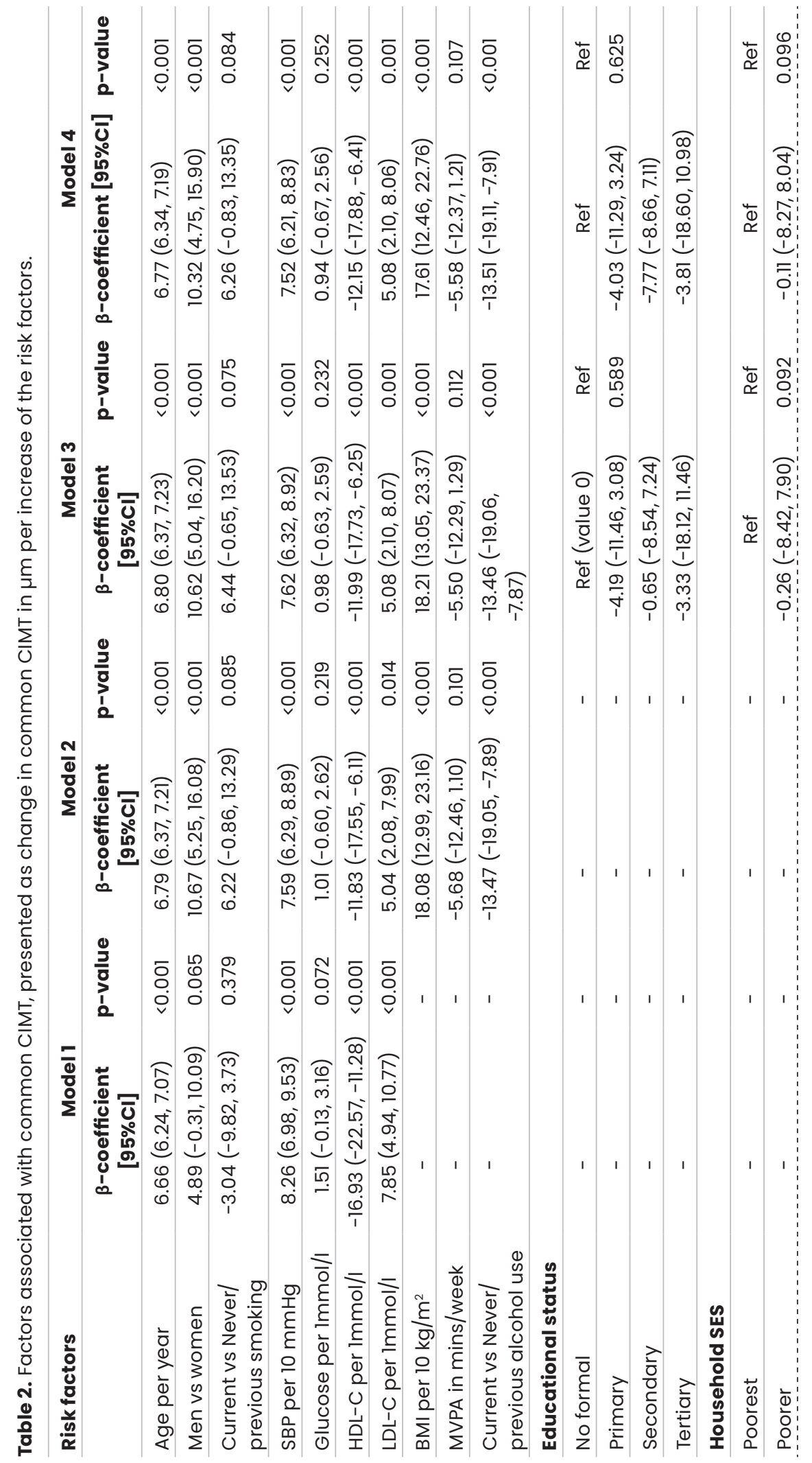




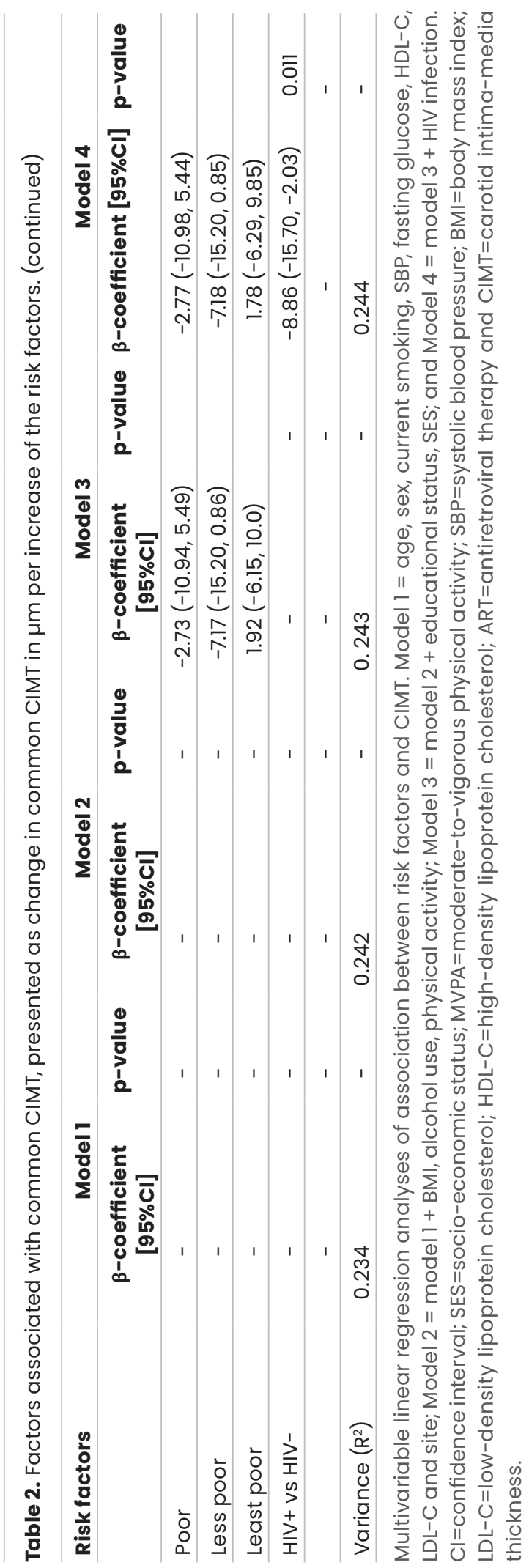




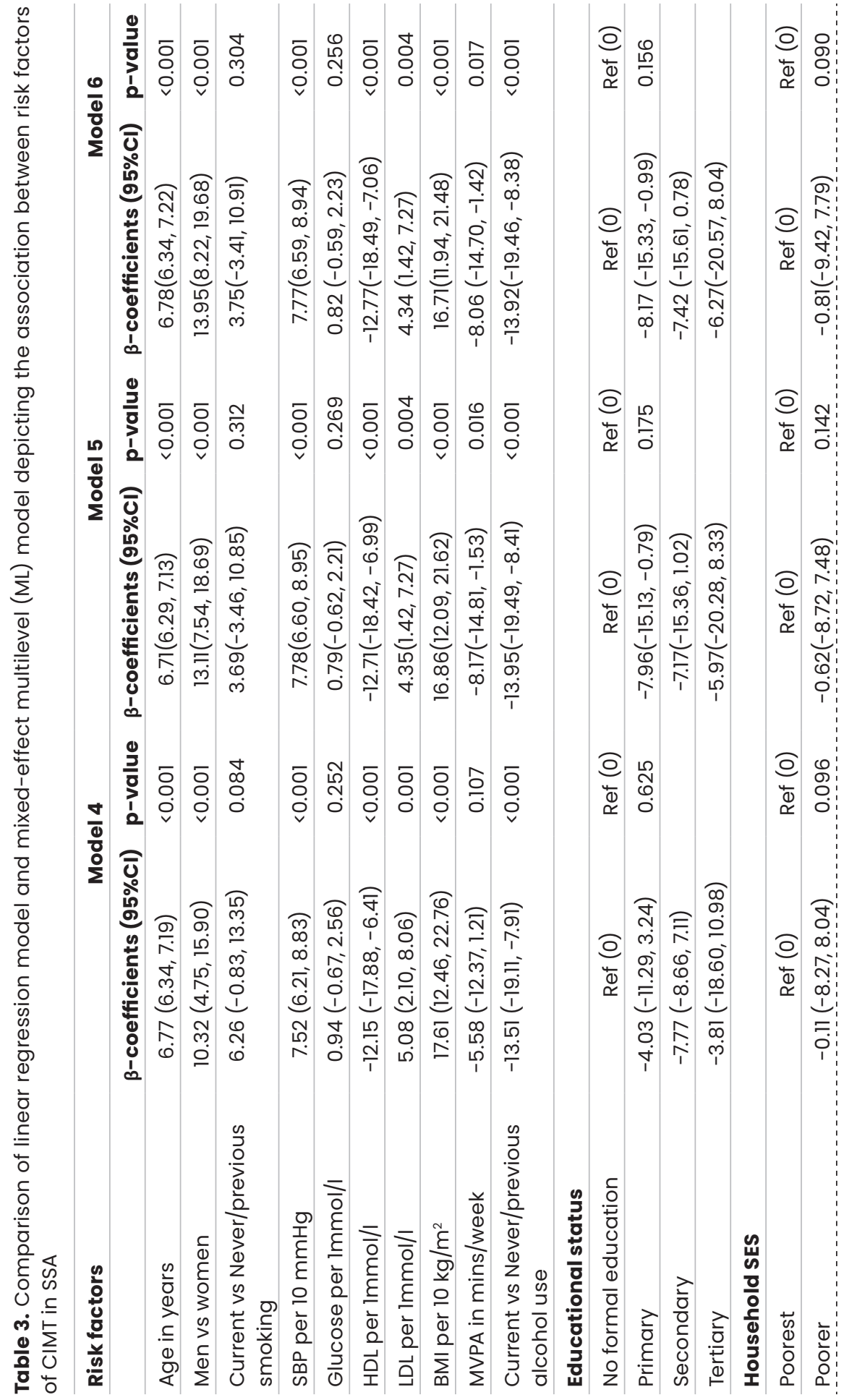


高

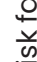

ब

$\sum_{0}^{\infty}$

요

$\frac{}{\frac{1}{}}$

음

of

$\stackrel{\oplus}{\perp}$

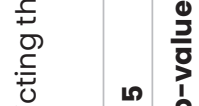

$\frac{0}{0}$

$\frac{1}{0}$

$\frac{1}{0}$

$\varepsilon$

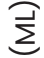

$\bar{\Phi}$

$\frac{\sqrt{\frac{0}{E}}}{\frac{2}{3}}$

ह

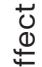

$\frac{1}{0}$

를

등

$\frac{1}{0}$

ํำ

음

$\frac{0}{2}$

ब)

$\bar{\partial}$

$\stackrel{ \pm}{=}$

응

ㄷำ

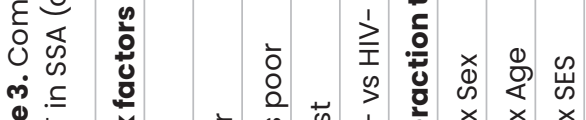

응 $\stackrel{\frac{5}{5}}{\frac{\underline{5}}{\frac{\omega}{\alpha}}}$

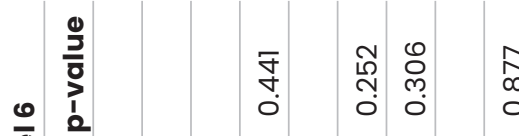

เั่

广 0 จิ

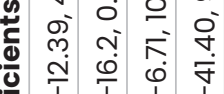

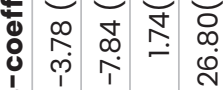

ํㅣㄹ

ปั

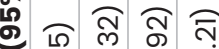

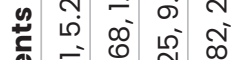

:

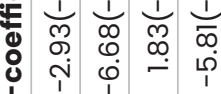

a

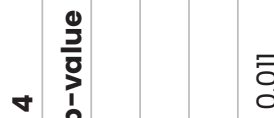

ฮ

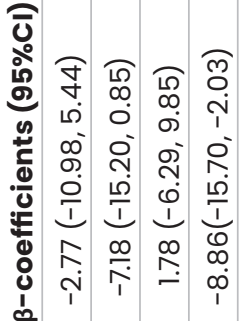

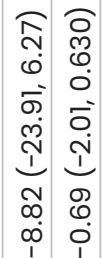

๑

స ल

ลิ่

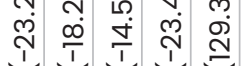

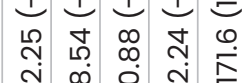

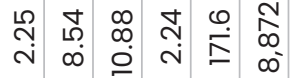

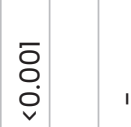

$>$

$\left|\begin{array}{l|l}\infty \\ 0 \\ 0\end{array}\right|$

응

की $\|$ ली

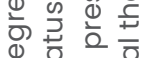

过

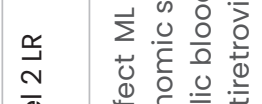

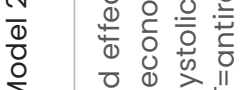

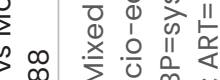

m)

要

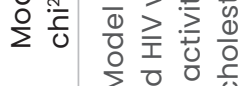

$\sum_{i=0}^{0} \frac{0}{0} \frac{c}{0}$

일 $\quad$ is

क ⿱艹

के

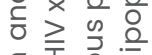

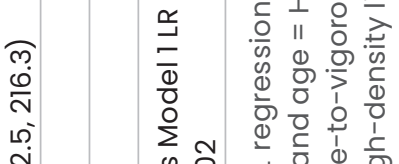

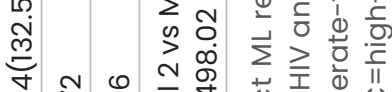

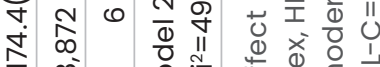

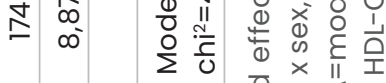

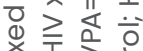

함

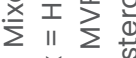

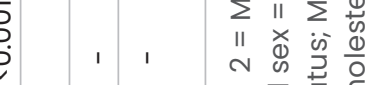

ब)

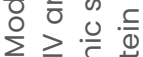

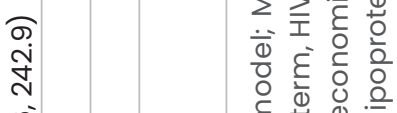

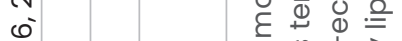

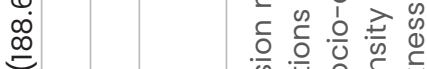

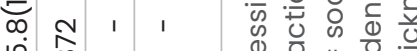

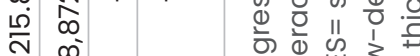

क्ष出出

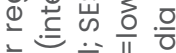

产 क

() ह \

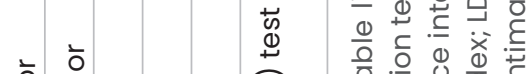

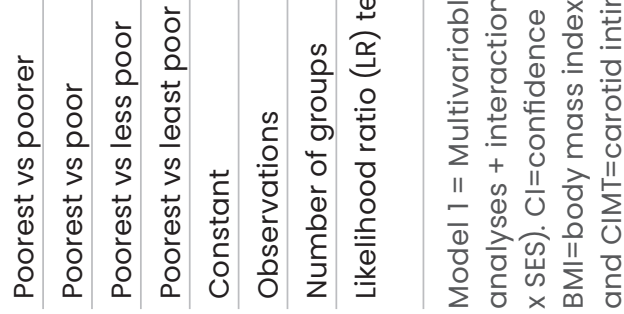


In sex stratified analyses (Tables 4 and 5), the observed association of age, SBP, $\mathrm{HDL}-\mathrm{C}$, current alcohol consumption and HIV infection with CIMT was similar between men and women. In the pooled analysis (Table 2), the association between smoking and CIMT had a trend towards a positive association (6.26 $\mu \mathrm{m}[-0.83,13.35] ; P=0.084)$. However, in men only current smoking was positively associated with higher CIMT $(10.7 \mu \mathrm{m}[2.93,18.53])$. Also in men but not women, a unit elevation in LDL-C (mmol/L) was likely to present with a $6.05 \mu \mathrm{m}$ [1.97, 10.13] higher CIMT, whilst higher household SES among men but not women was associated with a lower CIMT $(\mathrm{P}=0.042)$.

We added multiplicative interaction terms to the final model to evaluate whether relations between risk factors and common CIMT differed by sites (Table S2). We observed some significant interactions between site and certain risk factors. We therefore conducted sub-analyses for each site stratified by sex. In these site stratified analyses (Tables 4 and 5), differential effects of some of the independent variables on CIMT were observed. Significant associations of plasma glucose levels with CIMT were observed in women in Nanoro (-7.90 $[-13.8,-2.04])$ and Dikgale $(3.68[0.75,6.62])$ but the effect was negative in the former and positive in the latter group.

In women, BMI correlated significantly and negatively with CIMT in Navrongo $(-28.7[-52.9,-4.56])$ but positively in Dikgale $(17.8[7.34,28.2])$. Men with primary, secondary, and tertiary education in Soweto were likely to have higher CIMT than men with no formal education. Alcohol consumption was inversely associated with CIMT in men from Nanoro, Burkina Faso. Similarly, high HDL was associated with lower CIMT in women from Dikgale, South Africa and Nairobi, Kenya. The MVPA had similar inverse associations with CIMT in men from Navrongo and Burkina Faso (see Tables 4 and 5). 


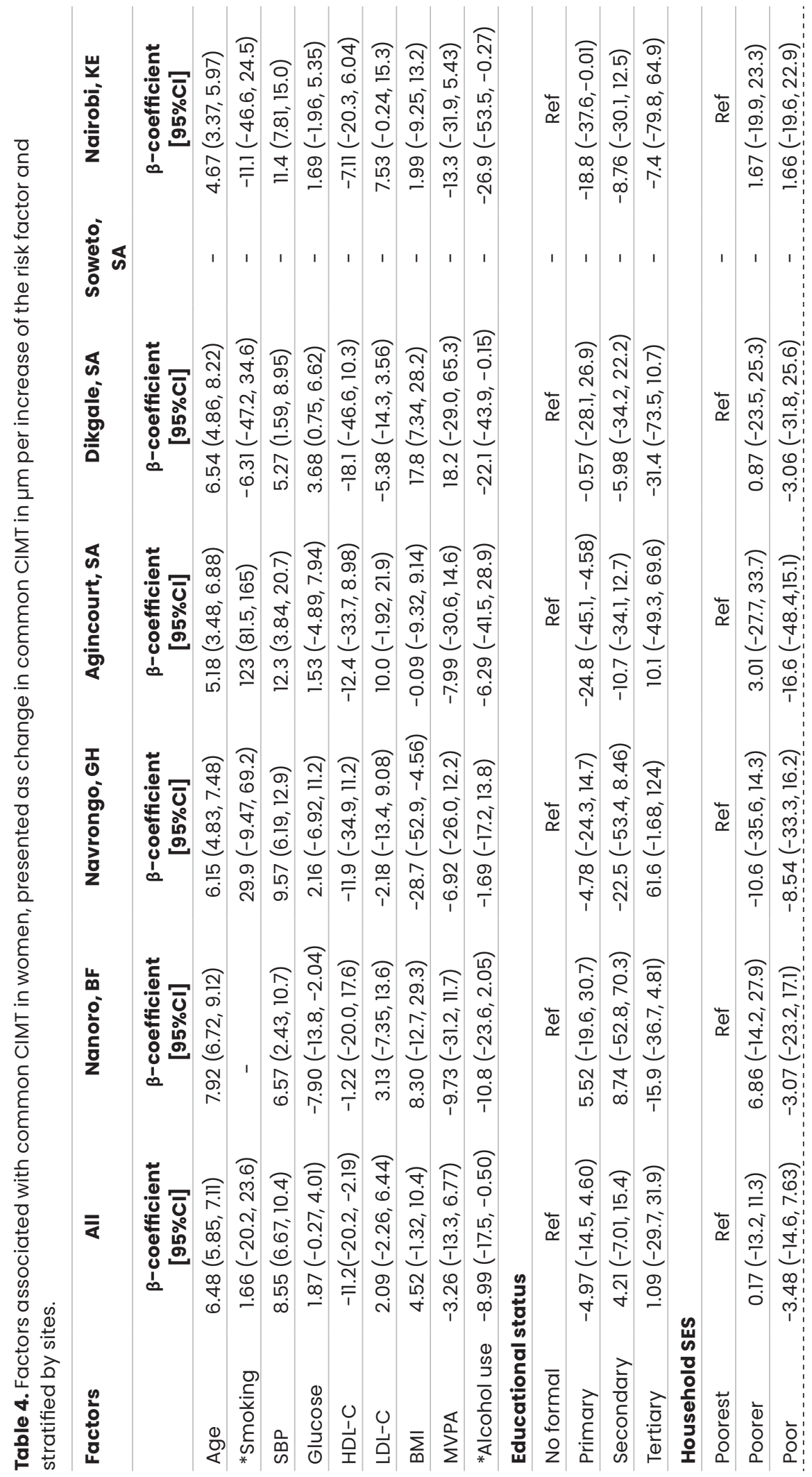




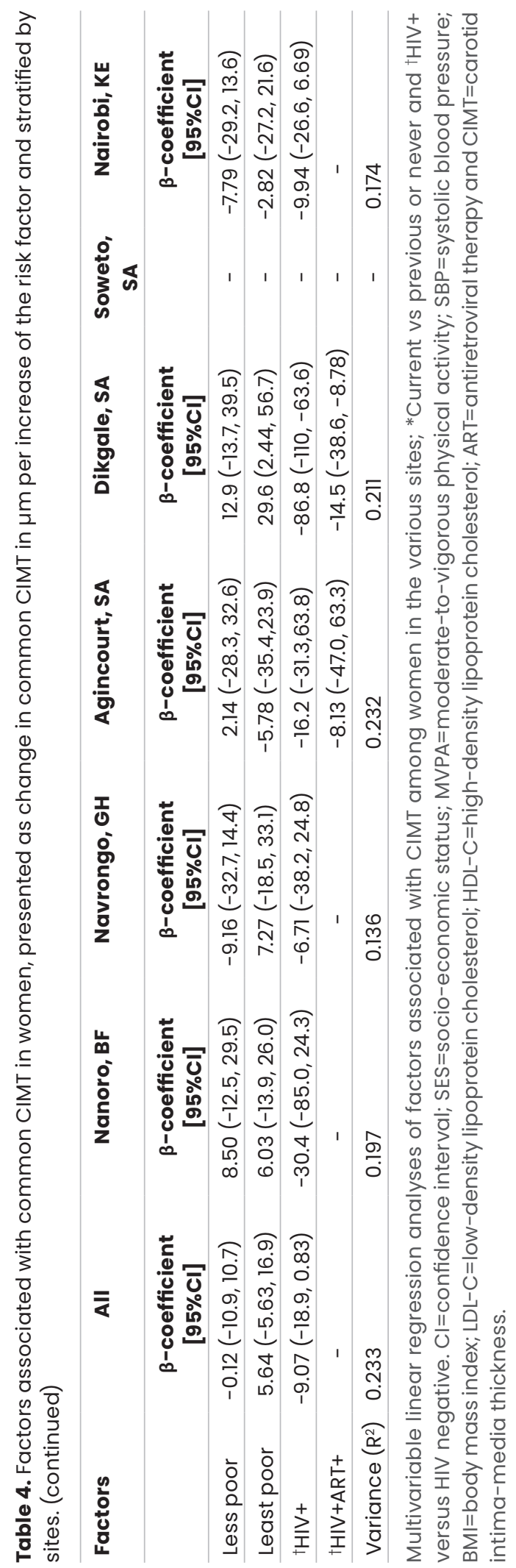


๘ ब) ○

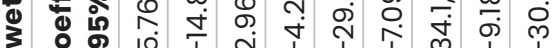
క. 0 0

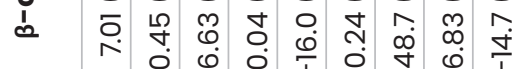

必

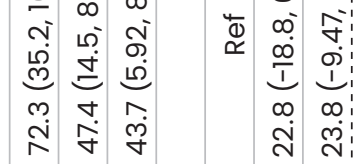

凹

สิ $ิ$

๑

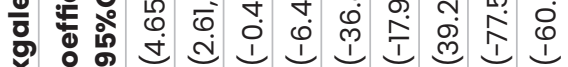

咅

กิ กั่

ก N

㐫

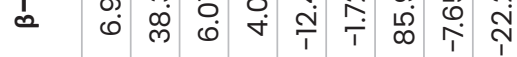

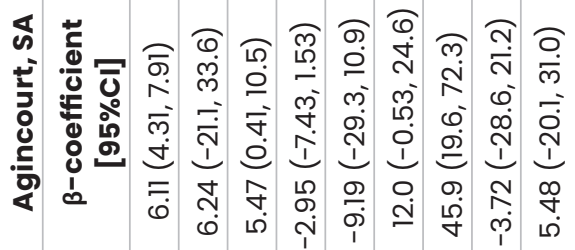

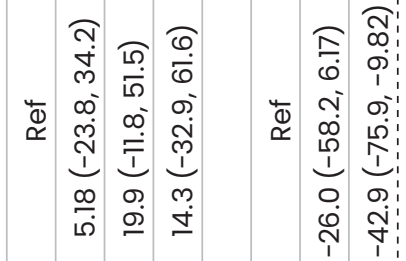

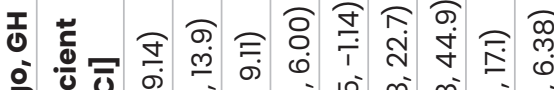

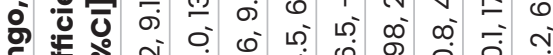
โ

ว 0 。

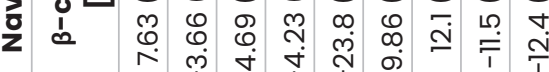

เ) ชอ

ลे

Ф)

จ mं

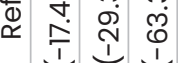

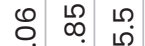

ம்

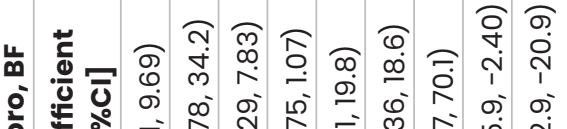

กิ

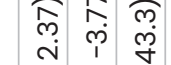

Ф)

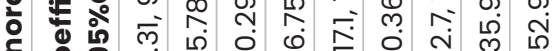

용

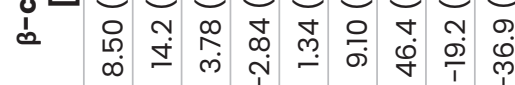

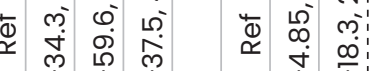

I 1 I

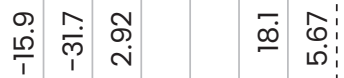

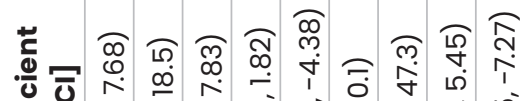

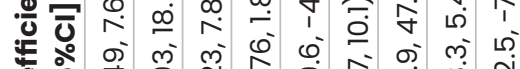

$\overline{\bar{\alpha}}$

ס ڤ

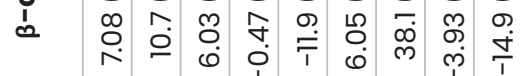

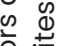




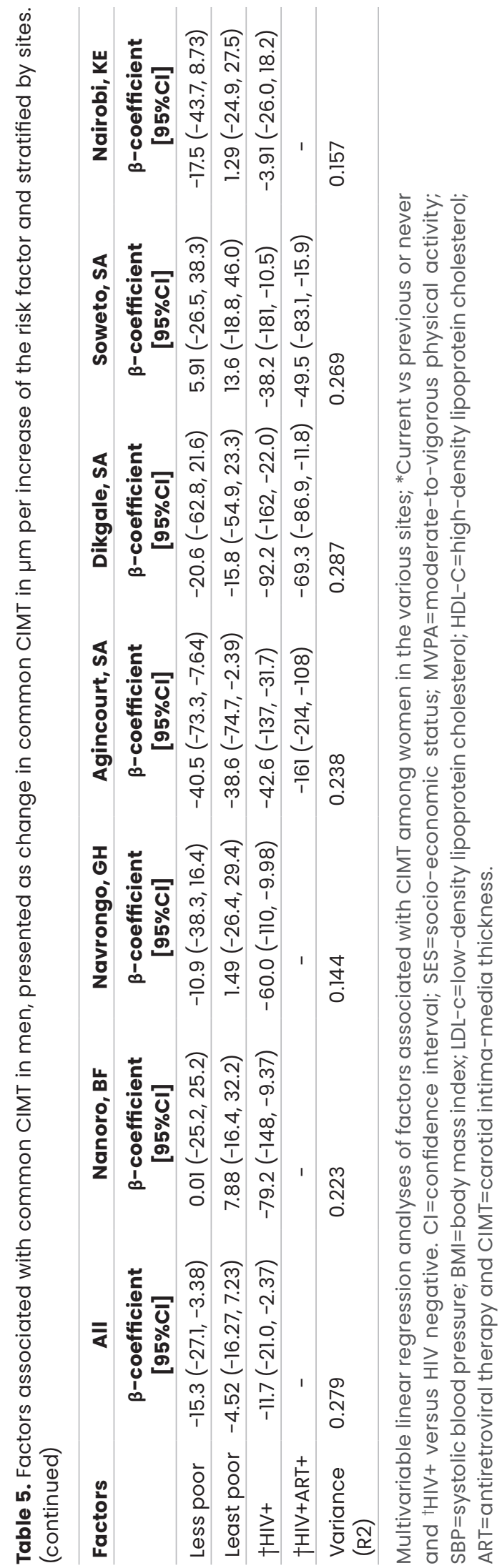




\section{Discussion}

The AWI-Gen study is the first large African study with harmonized data collection in 4 countries across 3 sub-continental African regions to report on the measurement of CIMT and its associated cardiometabolic risk factors. Our study shows both sex and regional differences in CIMT levels and risk factor associations. In pooled analyses, our findings show that the major factors that were consistently associated with higher CIMT were age, men, SBP, BMI, and $\mathrm{LDL}-\mathrm{C}$ while a high $\mathrm{HDL}-\mathrm{C}$, current alcohol consumption, and HIV infection were associated with lower CIMT. In a sex-stratified analysis, smoking was associated with a higher CIMT in men.

The mean CIMT of the left CCA was higher than the right and this has been reported previously in an adult Pakistani population (35). This could be because of the fact that the left CCA arises directly from the aortic arch and is therefore exposed to greater hemodynamic stress and intimal damage from the systolic pressure from the left ventricles. It may therefore be clinically relevant to use right CIMT for screening purposes. The mean CIMT of $0.64 \pm 0.003 \mathrm{~mm}$ in our study population was lower than $0.71 \pm 0.19 \mathrm{~mm}$ reported in adult populations from North America (36) and $0.71 \pm 0.12 \mathrm{~mm}$ in Europe (36). The difference is likely explained by the relatively older ages of the North American and European cohorts (mean ages 60.2 \pm 8.7 and 58.8 \pm 7.6 , respectively) compared with our population that had a mean age of $49.9 \pm 5.8$ years. The observed CIMT in our population was, however, higher than reported in studies from India (38), China (39), Pakistan (35), and South America $(40,41)$ which had a similar age to our population. These observed variations in mean CIMT between white, Asian, and African populations support the notion that CIMT varies by ethnicity4 and may therefore have a substantive genetic contribution, but it must also be noted that differences in sampling methods, sample size, ratio of men to women and CIMT measurement techniques across these studies may also play a part.

In the combined analysis, mean CIMT levels were similar for men and women. In Nanoro, Burkina Faso where women and men had similar ages, men had higher CIMT than women, whereas despite being younger, women had higher CIMT than men in Nairobi, Kenya. In other studies where women were observed to have higher CIMT compared with men, the differences were observed in women over the age of 45 years $(42,43)$. However, when we adjusted for other risk factors including age, men were more likely to present with higher CIMT compared with women. Differences in CIMT levels between men and women have been attributed to differences in exposure to CVD risk factors. ${ }^{44}$ In addition, physiological factors, such as lumen diameter, have been suggested as 
explanations for sex differences, with women generally presenting with smaller blood vessels (45).

The association between classical CVD risk factors and CIMT has been established in various populations, predominantly white and Asian (46-49) and only in a few studies, with small sample sizes, from African populations $(50,51)$. Classical CVD factors that have been observed to be associated with CIMT in the literature were similarly associated in the present study, and this provides some level of external validity to our data. Addition of other potential risk factors into a regression model that included these traditional CVD risk factors had a minimal effect on the variance in CIMT explained by the model. These risk factors included BMI, alcohol intake, and HIV, all of which did contribute significantly to the final model but slightly attenuated the association of the other risk factors with CIMT. Our findings are in agreement with the INTERHEART study that showed that, although the magnitude of the relations differed somewhat, established risk factors were associated with an increased risk of acute myocardial infarction across the world (52). These findings therefore suggest that interventions aimed at reducing the classical risk factors will help prevent the development of atherosclerosis and reduce CVDs and associated mortality in SSA populations.

The association of SBP with high CIMT levels is of particular interest as this presents a worrying phenomenon since a previous AWI-Gen study demonstrated a high prevalence of hypertension across all sites with an associated low awareness and control of high blood pressure (21). While we advocate for preventive measures that should be targeted to the local situation, early detection and management of hypertension may particularly benefit these SSA populations. Increased awareness, screening, early detection and subsequent management of CVD risk factors should be integrated into current public health systems in SSA.

We observed that current alcohol consumption was associated with lower CIMT and this is contrary to findings from the USE Intima-Media Thickness (USE-IMT) 8 cohorts collaboration conducted among North Americans and Europeans, which reported lower CIMT among participants who consumed little or no alcohol compared with those who consumed $>10 \mathrm{~g}$ of alcohol daily (53). A longitudinal study further clarified this by reporting that sustained heavy drinking in midlife was associated with higher CIMT (54). The observed inverse association of alcohol consumption with CIMT has also been documented in a Korean study (55). One possible explanation for this relationship comes from a randomized cross-over feeding trial among men which showed that alcohol improves lipid profiles and reduces atherosclerosis related inflammatory 
markers in plasma. The phenolic content of alcoholic drinks, which is high in sorghum-based beers, which are consumed widely in the study settings, was found to reduce leukocyte adhesion molecules and inflammatory biomarkers $(56,57)$. Furthermore, the ARIC (Atherosclerosis Risk in Communities) study, using candidate gene analyses with a Mendelian Randomization methodology, demonstrated that low-to-moderate alcohol consumption improves serum lipid levels (58). However, it should be noted that in the present study, the inverse association between alcohol intake and CIMT occurred independently of lipid levels. It is possible that the crude assessment of alcohol intake used in the current study may not be sensitive enough to allow more complex investigations of these relationships, and longitudinal studies using more objective measures of alcohol intake may be required.

In our population and according to the mixed-effect ML model, HIV infection showed a non-significant inverse association with lower CIMT after adjusting for age, education status, household SES, and the classical cardiovascular risk factors. This inverse association was shown to be significant in multivariable regression analyses. Both findings are contrary to studies from Brazil (59) and Uganda (60) reporting higher CIMT among HIV-positive compared with HIV-negative individuals. In line with these, a recent meta-analysis based on 17 cohort and case-control studies indicates a 2-fold higher risk ratio for cardiovascular diseases in people living with HIV in comparison with HIVnegative subjects. However, this meta-analysis included only 1 case-control study from Africa including 200 stroke cases and 398 controls. ${ }^{61}$ The nonsignificant association observed in the current study is similar to findings from rural South Africa (62), North America (63), and Brazil $(39,40)$. As HIV was not assessed objectively across all sites, with the exception of the South African and Kenyan sites, we conducted (multivariable linear regression) sub-analyses on data from these sites to assess the effect of HIV on CIMT. These showed a negative association between HIV and CIMT. It is interesting to note that a recent study conducted in rural South Africa among $(50,59)$ participants aged $\geq 40$ years has shown that HIV-positive subjects had a lower prevalence of classical CVD risk factors when compared with HIV-negative subjects, in both men and women (64). This has further been corroborated by more recent studies from South Africa, which demonstrated lower prevalence levels of hypertension and diabetes mellitus among HIV-infected subjects $(65,66)$. Considerably increased access to ART in SSA in the past decade, particularly so in South Africa, which has the largest ART roll-out program in the world (67), and application of treatment guidelines to screen people living with HIV for CVD risk $(32,68)$, may have prompted diagnosis and consequent treatment of identified CVD risk factors, or changes in lifestyle, in these subjects. These findings may well be indicative of an emerging paradigm reflecting an improved HIV care cascade. 
Further to this, studies have reported that the effects of HIV on CIMT are more pronounced in younger than older population because of active viral replication and immune activation and a greater level of high-CVD risk behaviours (69). Thus, potentially long-term use of ART as noted in our older population may result in chronic viral suppression leading to lower inflammation and reduced CIMT among HIV participants. However, it is possible that the negative association between HIV infection and CIMT observed in our study may be explained by other factors and thus, be attributed to residual confounding. Furthermore, misclassification of HIV status, particularly at sites that did not offer formal HIV testing could be possible, however, the low prevalence of HIV infection observed at such sites is supported by data from the literature $(29,30)$. It should also be noted that the level of ART coverage varied across the sites ranging from $44.6 \%$ in Soweto to $96.4 \%$ in Agincourt. Although data on ART regimens were not collected, treatment guidelines in Kenya (68) and South Africa (32) at the time of this study were similar with first line therapies consisting of tenofovir+lamivudine (3TC) + efavirenz/nevirapine and zidovudine (AZT)+lamivudine (3TC) + nevirapine/efavirenz. Despite the heterogeneity across the sites, the negative relationship between HIV infection and CIMT was observed at all study sites in both men and women, suggesting that this is a robust association. The paucity of data on the role of HIV and atherosclerotic CVDs in Africa therefore requires further studies with a wide range of HIVdefining markers, such as viral load, and a larger sample size of subjects with confirmed HIV and ART status.

Several variables demonstrated differential association with CIMT across the sites. Possible reasons for this may include differences across sites in: genetic variance, distribution of the particular effector variables and level of influence of modifying variables. These differential site-specific effects are important to understand but are beyond the scope of the current study and require in depth analysis at individual sites using larger sample sizes and a broader array of input variables.

Limitations to our study include a lack of information on the duration of HIV infection, the ART regimens in use and the duration of therapy, and the absence of measurements of viral load and CD4 counts, all of which may play a role in explaining the association between HIV infection and CIMT. Furthermore HIV status determined in the Agincourt site may have potentially resulted in measurement bias as participants' status may have changed from the time of initial HIV diagnosis to the time of actual recruitment for the AWI-Gen study. The main strength of our study relates to the fact that this is the first large scale study to determine CIMT levels and their association with cardiovascular risk factors in 3 sub-continental blocks in Africa. Measurements of most variables 
and CIMT were harmonized across all sites minimising variability and making pooled analysis and cross-site comparisons feasible. We were also able to cover the geographical and social variability across these regional blocks by recruiting in both rural and urban sites thus providing representation of the different stages of the epidemiological transition within Africa.

\section{Conclusions}

To our knowledge, this is the first large scale study from SSA to report on the levels of, and factors associated with, CIMT. We observed that the main drivers for higher CIMT were the same CVD risk factors associated with CIMT in white and Asian populations. Given the rising prevalence of these CVD risk factors in SSA, atherosclerotic diseases may become a major pan-African epidemic unless preventive measures are taken particularly targeted at prevention of hypertension and reduction in obesity. In addition, differential effects of certain factors on CIMT were observed across the SSA sites. We advocate for HIVspecific studies to fully understand the true association between HIV and CIMT in SSA.

\section{Appendix}

*H3Africa AWI-Gen Consortium Members Conception and design of the original AWI-Gen study: Michele Ramsay (SBIMB, Wits), Osman Sankoh (INDEPTH), Alisha Wade, Stephen Tollman and Kathleen Kahn (Agincourt), Marianne Alberts (Dikgale), Catherine Kyobutungi (Nairobi), Halidou Tinto (Nanoro), Abraham Oduro (Navrongo), Shane Norris (Soweto), and Scott Hazelhurst, Nigel Crowther, Himla Soodyall and Zane Lombard (Wits).

Site specific Investigators: Agincourt, South Africa (Frances Xavier GomezOlive Casas and Ryan Wagner), Dikgale, South Africa (Felistas Mashinya, Ian Cook and Sam Ntuli), Nairobi, Kenya (Christopher Khayeka-Wandabwa, Tilahun Nigatu Haregu, Shukri F. Mohammed and Stella Muthuri), Nanoro, Burkina Faso (Palwendé R. Boua, Herman Sorgho, Seydou Nakanabo-Diallo, Toussaint Rouamba), Navrongo, Ghana (Godfred Agongo, Cornelius Debpuur, Engelbert A. Nonterah, Eric Fato and Immaculate Anati and Lucas Amenga-Etego), Soweto, South Africa (Nomses Baloyi, Juliana Kagura, Richard Munthali and Yusuf Guman) and Wits AWI-Gen Collaborative Centre at SBIMB (Cassandra C. Soo, Freedom Mukomana, Stuart A. Ali, Ananyo Choudhury).

\section{Acknowledgments}

The AWI-Gen study would not have been possible without the generosity of the participants who spent many hours responding to questionnaires, being measured, and having samples taken. We wish to acknowledge the sterling 
contributions of our field workers, phlebotomists, laboratory scientists, administrators, data personnel and other staff who contributed to the data and sample collections, processing, storage, and shipping.

\section{Author Contributions}

Conception of paper (Nonterah, Klipstein-Grobusch, Bots and Crowther), data analysis (Nonterah), writing of original manuscript (Nonterah, PRB, KlipsteinGrobusch, Bots and Crowther), critical review and approval of the manuscript (all authors).

\section{Sources of Funding}

Nonterah is supported by a grant from the Global Health Support Program of the University Medical Center Utrecht (UMCU), University of Utrecht, The Netherlands and the Navrongo Health Research Centre (NHRC), Ghana. The AWIGen Collaborative Centre is funded by the National Human Genome Research Institute (NHGRI), Office of the Director (OD), Eunice Kennedy Shriver National Institute Of Child Health \& Human Development (NICHD), the National Institute of Environmental Health Sciences (NIEHS), the Office of AIDS Research (OAR) and the National Institute of Diabetes and Digestive and Kidney Diseases (NIDDK), of the National Institutes of Health under award number U54HG006938 and its supplements, as part of the H3Africa Consortium. Additional funding was leveraged from the Department of Science and Technology, South Africa, award number DST/CON 0056/2014. This paper describes the views of the authors and does not necessarily represent the official views of the National Institutes of Health (USA). Boua is funded by National Research Foundation/The World Academy of Sciences (NRF/TWAS) through the program: "African Renaissance Doctoral Fellowship."

\section{Disclosures}

None 


\section{References}

1. Roth GA, Johnson C, Abajobir A, Abd-Allah F, Abera SF, Bayou G, et al. Global, regional, and national burden of cardiovascular diseases for 10 causes, 1990 to 2015. J Am Coll Cardiol. 2017; 70:1-25.

2. GBD 2015 Risk Factors Collaborators. Global, regional, and national comparative risk assessment of 79 behavioural, environmental and occupational, and metabolic risks or clusters of risks, 1990-2015: a systematic analysis for the Global Burden of Disease Study 2015. Lancet. 2016; 388:1659-1724.

3. Libby P. Inflammation and atherosclerosis. Circulation. 2002; 105:1135-1143.

4. Bots ML, Evans GW, Tegeler $\mathrm{CH}$, Meijer R. Carotid intima-media thickness measurements: relations with atherosclerosis, risk of cardiovascular disease and application in randomized controlled trials. Chin Med J. 2016; 129:215- 226.

5. Touboul PJ, Hennerici MG, Meairs S, Adams H, Amarenco P, Bornstein N, et al. Mannheim carotid intima-media thickness and plaque consensus (2004-2006-2011). An update on behalf of the advisory board of the 3rd, 4th and 5th watching the risk symposia, at the 13th, 15th and 20th European Stroke Conferences, Mannheim, Germany, 2004, Brussels, Belgium, 2006, and Hamburg, Germany, 2011. Cerebrovasc Dis. 2012; 34:290-296.

6. Stein JH, Korcarz CE, Hurst RT, Lonn E, Kendall CB, Mohler ER, et al; American Society of Echocardiography Carotid Intima-Media Thickness Task Force. Use of carotid ultrasound to identify subclinical vascular disease and evaluate cardiovascular disease risk: a consensus statement from the American Society of Echocardiography Carotid Intima-Media Thickness Task Force. Endorsed by the Society for Vascular Medicine. J Am Soc Echocardiogr. 2008; 21:93-111.

7. Bauera M, Caviezelb S, Teynorb A, Raimund-Erbela R, Mahabadia AA, SchmidtTrucksässb A. Carotid intima-media thickness as a biomarker of subclinical atherosclerosis. Swiss Med Wkly. 2012; 142:1-9.

8. Chen LY, Leening MJ, Norby FL, Roetker NS, Hofman A, Franco OH, et al. Carotid intima-media thickness and arterial stiffness and the risk of atrial fibrillation: the Atherosclerosis Risk in Communities (ARIC) Study, Multi-Ethnic Study of Atherosclerosis (MESA), and the Rotterdam Study. J Am Heart Assoc. 2016; 5:e002907. DOI: 10.1161/JAHA.115.002907.

9. Villines TC, Hsu LL, Blackshear C, Nelson CR, Griswold M. Relation of carotid intimamedia thickness to cardiovascular events in Black Americans (from the Jackson Heart Study). Am J Cardiol. 2017; 120:1528-1532.

10. Ramsay M, Sankoh O; as members of the AWI-Gen study and the H3Africa Consortium. African partnerships through the H3Africa Consortium bring a genomic dimension to longitudinal population studies on the continent. Int J Epidemiol. 2016; 45:305-308.

11. Bawah A, Houle B, Alam N, Razzaque A, Streatfield PK, Debpuur C, et al. The evolving demographic and health transition in four low- and middle-income countries: evidence from four sites in the INDEPTH Network of Longitudinal Health and Demographic Surveillance Systems. PLoS One. 2016; 11:e0157281. 
12. Streatfield PK, Khan WA, Bhuiya A, Hanifi SM, Alam N, Bagagnan CH et al. Adult noncommunicable disease mortality in Africa and Asia: evidence from INDEPTH Health and Demographic Surveillance System sites. Glob Health Action. 2014; 7:25365.

13. Ramsay M, Crowther N, Tambo E, Agongo G, Baloyi V, Dikotope S, et al. H3Africa AWIGen Collaborative Centre: a resource to study the interplay between genomic and environmental risk factors for cardiometabolic diseases in four sub-Saharan African countries. Glob Health Epidemiol Genom. 2016; 1:e20.

14. Ali SA, Soo C, Agongo G, Alberts M, Amenga-Etego L, Boua RP, et al. Genomic and environmental risk factors for cardiometabolic diseases in Africa: methods used for Phase 1 of the AWI-Gen population cross-sectional study. Glob Health Action. 2018; 11:1507133.

15. Alberts M, Dikotope SA, Choma SR, Masemola ML, Modjadji SEP, Mashinya F, et al. Health $\&$ demographic surveillance system profile: the Dikgale health and demographic surveillance system. Int J Epidemiol. 2015; 44:1565-1571.

16. Kahn K, Collinson MA, Gomez-Olive FX, Mokoena O, Twine R, Mee P, et al. Profile: Agincourt health and socio-demographic surveillance system. Int J Epidemiol. 2012; 41:988- 1001.

17. Richter L, Norris S, Pettifor J, Yach D, Cameron N. Cohort profile: Mandela's children: the 1990 Birth to Twenty study in South Africa. Int J Epidemiol. 2007; 36:504-511.

18. Beguy D, Elung'ata P, Mberu B, Oduor C, Wamukoya M, Nganyi B, Ezeh A. Health \& demographic surveillance system profile: the Nairobi urban health and demographic surveillance system (NUHDSS). Int J Epidemiol. 2015; 44:462-471.

19. Oduro AR, Wak G, Azongo D, Debpuur C, Wontuo P, Kondayire F, et al. Profile of the Navrongo health and demographic surveillance system. Int J Epidemiol. 2012; 41:968976.

20. Derra K, Rouamba E, Kazienga A, Ouedraogo S, Tahita MC, Sorgho H, et al. Profile: Nanoro health and demographic surveillance system. Int J Epidemiol. 2012; 41:12931301.

21. Gomez-Olive FX, Ali SA, Made F, Kyobutungi C, Nonterah E, Micklesfield L, et al. Regional and sex differences in the prevalence and awareness of hypertension: an H3Africa AWI-Gen Study across 6 sites in sub-Saharan Africa. Glob Heart. 2017; 12:81-90.

22. Klipin M, Mare I, Hazelhurst S, Kramer B. The process of installing REDCap, a web based database supporting biomedical research: the first year. Appl Clin Inform. 2014; 5:916-929.

23. Harris PA, Taylor R, Thielke R, Payne J, Gonzalez N, Conde JG. Research Electronic Data Capture (REDCap) - a metadata-driven methodology and workflow process for providing translational research informatics support. J Biomed Inform. 2009; 42:377-381.

24. Nambi V, Chambless L, He M, Folsom AR, Mosley T, Boerwinkle E, Ballantyne CM. Common carotid artery intima-media thickness is as good as carotid intima-media thickness of all carotid artery segments in improving prediction of coronary heart disease risk in the Atherosclerosis Risk in Communities (ARIC) study. Eur Heart J. 2012; 33:183-190. 
25. Ewing JA. Detecting alcoholism: the CAGE questionnaire. JAMA. 1984; 252: 1905-1907.

26. Bull FC, Maslin TS, Armstrong T. Global physical activity questionnaire (GPAQ): nine country reliability and validity study. J Phys Act Health. 2009; 6:790-804.

27. Friedewald WT, Levy RI, Fredrickson DS. Estimation of the concentration of low-density lipoprotein cholesterol in plasma, without use of the preparative ultracentrifuge. Clin Chem. 1972; 18:499-502.

28. Gomez-Olive FX, Montana L, Wagner RG, Kabudula CW, Rohr JK, Kahn K, et al. Cohort profile: health and ageing in Africa: a longitudinal study of an INDEPTH community in South Africa (HAALSI). Int J Epidemiol. 2018; 47:689-690j.

29. National AIDS/STI Control Programme. HIV Sentinel Survey Report, 2015 Ghana Health Service. 2016. Available at: http://www.ccmghana.net/index.php/ surveys?download=113:hiv-sentinel-survey-2015. Accessed April 5, 2018.

30. WHO. Country cooperation strategy at a glance. Geneva, Switzerland: World Health Organisation; 2016. Available at: http://apps.who.int/gho/data/node.cco. Accessed April 4, 2018.

31. Preiss D, Kristensen SL. The new pooled cohort equations risk calculator. Can J Cardiol. 2015; 31:613-619.

32. Meintjes G, Maartens G, Boulle A, Conradie F, Goemaere E, Hefer E, et al. Guidelines for antiretroviral therapy in adults by the Southern African HIV Clinicians Society. South Afr J HIV Med. 2012; 13:114.

33. Kwan GF, Mayosi BM, Mocumbi AO, Miranda JJ, Ezzati M, Jain Y, et al. Endemic cardiovascular diseases of the poorest billion. Circulation. 2016; 133:2561-2575.

34. Stringhini S, Bovet P. Socioeconomic status and risk factors for noncommunicable diseases in low-income and lower-middle-income countries. Lancet Glob Health. 2017; 5:e230-e231.

35. Waseem M, Mubashir AA, Arif A, Vaqar B, Mirza KA, Kainat F. Carotid intima media thickness percentiles for Pakistani population. J Coll Physicians Surg Pak. 2017; 27:584-586.

36. Bauer M, Delaney JA, Mohlenkamp S, Jockel KH, Kronmal RA, Lehmann N, et al. Comparison of factors associated with carotid intima-media thickness in the MultiEthnic Study of Atherosclerosis (MESA) and the Heinz Nixdorf Recall Study (HNR). J Am Soc Echocardiogr. 2013; 26:667-673.

37. Grimaud O, Lapostolle A, Berr C, Helmer C, Dufouil C, Kihal W, et al. Gender differences in the association between socioeconomic status and subclinical atherosclerosis. PLoS One. 2013; 8:e80195.

38. Kasliwal RR, Bansal M, Desai N, Kotak B, Raza A, Vasnawala H, et al. A study to derive distribution of carotid intima media thickness and to determine its Correlation with cardiovascular Risk factors in asymptomatic nationwide Indian population (SCOREIndia). Indian Heart J. 2016; 68:821-827.

39. Liu B, Ni J, Shi M, Bai L, Zhan C, Lu H, et al. Carotid intima-media thickness and its association with conventional risk factors in low-income adults: a population-based cross-sectional study in China. Sci Rep. 2017; 7:41500. 
40. Pacheco AG, Grinsztejn B, da Fonseca MDE J, Moreira RI, Veloso VG, Friedman RK, et al. Traditional risk factors are more relevant than HIV-specific ones for carotid intimamedia thickness (cIMT) in a Brazilian cohort of HIV-infected patients. PLoS One. 2015; 10:e0117461.

41. Pacheco AG, Grinsztejn B, Fonseca Mde J, Griep RH, Lotufo P, Bensenor I, et al. HIV infection is not associated with carotid intima-media thickness in Brazil: a crosssectional analysis from the INI/ELSA-Brasil Study. PLoS One. 2016; 11:e0158999.

42. Ouyang P, Vaidya D, Dobs A, Golden SH, Szklo M, Heckbert SR, et al. Sex hormone levels and subclinical atherosclerosis in postmenopausal women: the Multi-Ethnic Study of Atherosclerosis. Atherosclerosis. 2009; 204:255-261.

43. Zhou Y, Wang D, Yang X, Wang A, Gao X, Guo Y, et al. Effect of menopausal status on carotid intima-media thickness and presence of carotid plaque in Chinese women generation population. Sci Rep. 2015; 5:8076.

44. Yusuf S, Reddy S, ôunpuu S, Anand S. Global burden of cardiovascular diseases part I: general considerations, the epidemiologic transition, risk factors, and impact of urbanization. Circulation. 2001; 104:2746-2753.

45. Bots ML, Hoes A, Koudstaal PJ, Hofman A, Grobbee DE. Common carotid intimamedia thickness and risk of stroke and myocardial infarction: the Rotterdam Study. Circulation. 1997; 96:1432-1437.

46. Baroncini LAV, de Castro Sylvestre L, Filho RP. Carotid intima-media thickness and carotid plaque represent different adaptive responses to traditional cardiovascular risk factors. Int J Cardiol Heart Vasc. 2015; 9:48-51.

47. Gao L, Bai L, Shi M, Ni J, Lu H, Wu Y, et al. Association between carotid intima-media thickness and fasting blood glucose level: a population-based cross-sectional study among low-income adults in rural China. J Diabetes Investig. 2017; 8:788-797.

48. Ferreira JP, Girerd N, Bozec E, Machu JL, Boivin JM, London GM, et al. Intima-media thickness is linearly and continuously associated with systolic blood pressure in a population-based cohort (STANISLAS Cohort Study). J Am Heart Assoc. 2016; 5:e003529. DOI: 10.1161/JAHA.116.003529.

49. Loboz-Rudnicka M, Jaroch J, Bociaga Z, Rzyczkowska B, Uchmanowicz I, Polanski J, et al. Impact of cardiovascular risk factors on carotid intima-media thickness: sex differences. Clin Interv Aging. 2016; 11:721-731.

50. Omisore AD, Famurewa OC, Komolafe MA, Asaleye CM, Fawale MB, Afolabi BI. Association of traditional cardiovascular risk factors with carotid atherosclerosis among adults at a teaching hospital in south-western Nigeria. Cardiovasc J Afr. 2018; 29:1-7.

51. Owolabi MO, Agunloye AM, Umeh EO, Akpa OM. Can common carotid intima media thickness serve as an indicator of both cardiovascular phenotype and risk among black Africans? Eur J Prev Cardiol. 2015; 22: 1442-1451.

52. Yusuf S, Hawken S, ôunpuu S, Dans T, Avezum A, Lanas F, et al; INTERHEART Study Investigators. Effect of potentially modifiable risk factors associated with myocardial infarction in 52 countries (the INTERHEART study): case-control study. Lancet. 2004; 364:937-952. 
53. Britton AR, Grobbee DE, den Ruijter HM, Anderson TJ, Desvarieux M, Engström G, et al. Alcohol consumption and common carotid intima-media thickness: the USE-IMT study. Alcohol Alcohol. 2017; 52:483-486.

54. Britton A, Hardy R, Kuh D, Deanfield J, Charakida M, Bell S. Twenty-year trajectories of alcohol consumption during midlife and atherosclerotic thickening in early old age: findings from two British population cohort studies. BMC Med. 2016; 14:111.

55. Lee YH, Shin MH, Kweon SS, Choi SW, Kim HY, Ryu SY, et al. Alcohol consumption and carotid artery structure in Korean adults aged 50 years and older. BMC Public Health. 2009; 9:358.

56. Chiva-Blanch G, Magraner E, Condines X, Valderas-Martínez P, Roth I, Arranz S, et al. Effects of alcohol and polyphenols from beer on atherosclerotic biomarkers in high cardiovascular risk men: a randomized feeding trial. Nutr Metab Cardiovasc Dis. 2015; 25:36-45.

57. WHO. Global status report on alcohol and health. Geneva, Switzerland: World Health Organisation; 2014. Available at: https://www.who.int/substance_ab use/ publications/global_alcohol_report/en/. Accessed March 10, 2018.

58. Vu KN, Ballantyne CM, Hoogeveen RC, Nambi V, Volcik KA, Boerwinkle E, et al. Causal role of alcohol consumption in an improved lipid profile: the Atherosclerosis Risk in Communities (ARIC) study. PLoS One. 2016; 11:1-16.

59. Godoi ET, Brandt CT, Lacerda HR, Godoi JT, Oliveira DC, Costa GF, et al. Intima-media thickness in the carotid and femoral arteries for detection of arteriosclerosis in human immunodeficiency virus-positive individuals. Arq Bras Cardiol. 2017; 108:3-11.

60. Ssinabulya I, Kayima J, Longenecker C, Luwedde M, Semitala F, Kambugu A, et al. Subclinical atherosclerosis among HIV-infected adults attending HIV/AIDS care at two large ambulatory HIV clinics in Uganda. PLoS One. 2014; 9:e89537.

61. Shah ASV, Stelzle D, Lee KK, Beck EJ, Alam S, Clifford S, Longenecker CT, et al. Global burden of atherosclerotic cardiovascular diseases in people living with HIV - systemic review and meta-analyses. Circulation. 2018; 138:1100-1112.

62. Schoffelen AF, de Groot E, Tempelman HA, Visseren FL, Hoepelman Al, Barth RE. Carotid intima media thickness in mainly female HIV-infected subjects in rural South Africa: association with cardiovascular but not HIV-related factors. Clin Infect Dis. 2015; 61:1606-1614.

63. Hanna DB, Guo M, Buzkova P, Miller TL, Post WS, Stein JH, et al. HIV infection and carotid artery intima-media thickness: pooled analyses across 5 cohorts of the NHLBI HIVCVD Collaborative. Clin Infect Dis. 2016; 63:249-256.

64. Gaziano TA, Abrahams-Gessel S, Gomez-Olive FX, Wade A, Crowther NJ, Alam S, et al. Cardiometabolic risk in a population of older adults with multiple co-morbidities in rural South Africa: the HAALSI (Health and Aging in Africa: longitudinal studies of INDEPTH communities) study. BMC Public Health. 2017; 17:206.

65. Manne-Goehler J, Siedner MJ, Montana L, Harling G, Geldsetzer P, Rohr J, et al. Hypertension and diabetes control along the HIV care cascade in rural South Africa. J Int AIDS Soc. 2019; 22:e25213. 
66. Manne-Goehler J, Montana L, Gomez-Olive FX, Rohr J, Harling G, Wagner RG, et al. The ART advantage: health care utilization for diabetes and hypertension in rural South Africa. J Acquir Immune Defic Syndr. 2017; 75:561-567.

67. South African National AIDS Council. 'Global AIDS Response Progress Report'. 2015. Available at: http://sanac.org.za/2016/06/22/global-aids-responseprogress-reportgarpr-2015/. Accessed May 13, 2019.

68. National AIDS/STI Control Program (NASCOP). Guidelines for antiretroviral therapy in Kenya. 4th ed. Nairobi, Kenya: 2011. Available at: http://guidelines.health.go. ke:8000/ media/Final_guidelines_re_print_11-09-2012.pdf. Accessed April 13, 2019.

69. Freiberg MS, So-Armah K. HIV and cardiovascular disease: we need a mechanism, and we need a plan. J Am Heart Assoc. 2016; 4:e003411. DOI: 10. 1161/JAHA.116.003411. 


\section{Supplementary material}

Table S1. Univariable estimates for exposure variables and CIMT in the final combined model

\begin{tabular}{|c|c|c|}
\hline Risk factors & $\beta$-coefficient $[95 \% \mathrm{Cl}]$ & P-value \\
\hline Age (years) & $7.91[7.49,8.33]$ & $<0.001$ \\
\hline Men vs women & $0.99[-4.12,6.11]$ & 0.704 \\
\hline Current vs Never/previous smoking & $-11.93[-18.45,-5.46]$ & $<0.001$ \\
\hline SBP per $10 \mathrm{mmHg}$ & $10.49[9.22,11.75]$ & $<0.001$ \\
\hline Glucose per 1mmol/l & $0.50[-1.17,2.18]$ & 0.556 \\
\hline $\mathrm{HDL}-\mathrm{C}$ per $1 \mathrm{mmol} / \mathrm{I}$ & $-20.24[-26.34,-14.15]$ & $<0.001$ \\
\hline LDL-C per lmmol// & $-4.04[-7.05,-1.04]$ & 0.008 \\
\hline BMI per $10 \mathrm{~kg} / \mathrm{m}^{2}$ & $-2.30[-6.40,1.79]$ & 0.271 \\
\hline MVPA in mins/week & $-17.85[-25.42,-10.270$ & $<0.001$ \\
\hline Current vs Never/previous alcohol use & $15.03[9.91,20.15]$ & $<0.001$ \\
\hline \multicolumn{3}{|l|}{ Educational status } \\
\hline No formal education & Ref & \\
\hline Primary & $-49.29[-55.52,-43.03]$ & $<0.001$ \\
\hline Secondary & $-58.97[-64.99,-52.96]$ & \\
\hline Tertiary & $-49.92[-64.52,-35.33]$ & \\
\hline \multicolumn{3}{|l|}{ Household SES } \\
\hline Poorest & Ref & \\
\hline Poorer & $-8.37[-17.37,0.63]$ & 0.052 \\
\hline Poor & $-8.93[-17.94,0.08]$ & \\
\hline Less poor & $-11.11[-19.93,-2.29]$ & \\
\hline Least poor & $-2.63[-11.26,6.00]$ & \\
\hline HIV+ vs HIV- & $-57.31[-63.95,-50.68]$ & $<0.001$ \\
\hline
\end{tabular}


Table S2. Multiplicative interaction terms between sex and site with the risk factors

\begin{tabular}{lc}
\hline Site and risk factor interaction terms & p-value \\
\hline Site with Age & $<0.001$ \\
\hline Site with Sex & $<0.001$ \\
\hline Site with Current smoking & 0.844 \\
\hline Site with Systolic blood pressure & 0.043 \\
\hline Site with Glucose & 0.079 \\
\hline Site with HDL-C & 0.619 \\
\hline Site with LDL-C & 0.078 \\
\hline Site with BMI & $<0.001$ \\
\hline Site with MVPA & 0.299 \\
\hline Site with Current alcohol & 0.281 \\
\hline Site with Educational status & 0.003 \\
\hline Site with Household SES & 0.108 \\
\hline Site with HIV status & 0.113 \\
\hline
\end{tabular}

SES=socio-economic status; MVPA=moderate-to-vigorous physical activity; BMI=body mass index; $\mathrm{LDL}-\mathrm{C}=\mathrm{low}$-density lipoprotein cholesterol; $\mathrm{HDL}-\mathrm{C}=$ high-density lipoprotein cholesterol 
Subclinical atherosclerosis in African populations 


\section{Abstract}

Background: Obesity and adipose tissue distribution contribute to an increased risk of cardiovascular disease (CVD) by promoting atherosclerosis. This association has been poorly studied in sub-Saharan Africa (SSA) despite the rising prevalence of cardiovascular disease.

Objectives: We determined the association between various adiposity phenotypes and carotid intima-media thickness (CIMT), a proxy of subclinical atherosclerosis, in a large SSA population.

Methods: A population-based cross-sectional study was performed from 20132016 in Burkina Faso, Ghana, Kenya and South Africa. Body mass index (BMI), waist (WC), hip circumferences (HC), visceral (VAT) and subcutaneous adipose tissue (SCAT) using B-mode ultrasound were measured. Ultrasonography of left and right far wall CIMT of the common carotid artery was used as an indicator of subclinical atherosclerosis. Individual participant data meta-analyses were used to determine the associations between adiposity phenotypes and CIMT in the pooled sample while adjusted multivariable linear regression analyses were used for site specific analyses.

Results: Data were obtained from 9,010 adults $(50.3 \%$ women and a mean age of $50 \pm 6$ years). Men had higher levels of visceral fat than women while women had higher BMI, waist and hip circumference and subcutaneous fat than men at all sites except Burkina Faso. In the pooled analyses, BMI ( $\beta$-value [ $95 \% \mathrm{Cls}]$ : $19.5[16.8,22.3] \mu \mathrm{m})$ showed the strongest relationship with CIMT followed by VAT $(5.86[4.65,7.07] \mu \mathrm{m}), \operatorname{SCAT}(5.00[2.85,7.15] \mu \mathrm{m}), \mathrm{WC}(1.27[1.09,1.44] \mu \mathrm{m})$ and $\mathrm{HC}$ $(1.23[1.04,1.42] \mu \mathrm{m})$. Stronger associations were observed in men than in women.

Conclusion: Obesity within SSA will likely result in higher levels of atherosclerosis and promote the occurrence of cardio- and cerebrovascular events, especially in males, unless addressed through primary prevention of obesity in both rural and urban communities across Africa. The inverse association of VAT with CIMT in Burkina Faso and Ghana requires further investigation.

Keywords: Carotid intima-media thickness; subclinical atherosclerosis; obesity; adiposity; cardiovascular disease; sub-Saharan Africa 


\section{Highlights}

- All adiposity phenotypes were positively associated with common carotid intima-media thickness (CIMT) in the entire cohort (pooled analyses).

- BMI had the strongest association with CIMT compared to other phenotypes.

- The magnitude of association between adiposity phenotypes and CIMT was higher in men than in women.

- Subcutaneous adipose tissue was inversely associated with CIMT only in women.

- An unexpected finding was the inverse association of visceral adipose tissue with CIMT in Burkina Faso and Ghana.

\section{Introduction}

Obesity is a major epidemic that occurs not only in the western world. Recent data from sub-Saharan Africa (SSA) demonstrate an equally high burden $(1,2)$, with south and north Africa having the highest prevalence levels (3). Projections from the World Health Organization (WHO) suggest that in 2025, $75 \%$ of the world's obese population will be in low- and middle-income countries such as those in SSA $(4,5)$. The recent Non-Communicable Disease (NCD) Risk Factor Collaboration study of 112 million adults further observed that the rising prevalence of obesity in rural communities contributes significantly to the global obesity epidemic (6). This is a major health concern, because obesity promotes the development of diabetes mellitus, is related to unfavorable levels of established cardiovascular risk factors such as dyslipidemia and hypertension, promotes the development of atherosclerosis and contributes to an increase in risk of symptomatic cardiovascular events $(7,8)$.

Several measures of adiposity are available to assess various overall and central body fat phenotypes. Thus, for general obesity, body mass index (BMI) is the most common measure while total body fat can be measured using dualenergy X-ray absorptiometry (DXA). Central obesity is often assessed using waist circumference (WC), with specific abdominal fat depots i.e., visceral (VAT) and subcutaneous (SCAT) adipose tissue assessed using imaging techniques such as magnetic resonance imaging (MRI), computerized tomography (CT) and B-mode ultrasonography $(9,10)$. Peripheral or lower body obesity, particularly fat tissue in the gluteofemoral region, is commonly measured using hip circumference ( $\mathrm{HC}$ ) whilst gluteofemoral fat can be specifically measured using the imaging methodologies described above. These adiposity phenotypes have been suggested to exert different effects on the development 
of CVD risk factors and atherosclerosis (11). Body fat distribution has been shown to be different in black Africans compared to their white counterparts $(12,13)$.

The relationship between obesity and atherosclerosis can be investigated by the use of carotid intima-media thickness (CIMT) of the common carotid artery (CCA). Extensive research has shown that common CIMT, assessed using B-mode ultrasonography, is a marker of the presence of atherosclerosis locally and elsewhere in the arterial system, and, as such, is an intermediate stage for the development of CVDs (14-16). However, most of the research examining the relationship of adiposity with atherosclerosis, assessed using common CIMT has been conducted in Whites (17), Asians (18-20) and African-Americans (21) from high income countries, but little information is available for black African populations from SSA.

In the present study, we used data from the Africa-Wits-INDEPTH [International Network for the Demographic Evaluation of Populations and Their Health] Partnership for Genomic Studies (AWI-Gen) project (22) to determine the relationship of various adiposity phenotypes with CIMT in four SSA countries Kenya, Ghana, Burkina Faso and South Africa.

\section{Methods}

\section{Study design, setting and participants}

The AWI-Gen study is embedded within the $\mathrm{NIH}$-funded Human Heredity and Health in Africa (H3Africa) Consortium. AWI-Gen is a population-based longitudinal study conducted in six sites in four SSA countries with baseline data collection occurring between 2013 and 2016 as described previously $(23,24)$. The study was conducted at five Health and Socio-Demographic Surveillance Sites (HDSS) under the INDEPTH Network and the MRC/Wits Developmental Pathways to Health Research Unit (DPHRU) in Soweto, South Africa (25). The HDSS sites in South Africa were the cohort from Bushbuck Ridge in Mpumalanga (referred to as Agincourt HDSS in this publication) (26) and Dikgale HDSS (27). The other sites were in East Africa: African Population and Health Research Center HDSS, Nairobi, Kenya (28) and two rural sites in West Africa: Nanoro HDSS, Burkina Faso (29) and the Navrongo HDSS, Ghana (30). These countries are located in three subregional African blocks and may be representative of the social, geographical and genetic diversity of SSA. Study participants were adult women and men aged 40-60 years living in the various sites. Details of the sampling methods and recruitment strategies used by the various sites have been described in a previous publication (24). 


\section{Ethical considerations}

The AWI-Gen study received ethics approval from the Human Research Ethics Committee (HREC) of the University of the Witwatersrand, Johannesburg, South Africa (Ethics approval identification number: M121029, renewed in 2017 with number: M170880). Additional ethics approvals were obtained from the national and institutional ethics boards/committees of the University of Limpopo, Nanoro HDSS, Burkina Faso; Nairobi HDSS, Kenya and Navrongo HDSS, Ghana. Written informed consent was obtained from all participants prior to recruitment.

\section{Data availability}

The datasets generated and/or analyzed during the current study will be made publicly available in the European Genome-phenome Archive under the set of projects related to the Human Heredity and Health in Africa (H3Africa) Consortium. Details concerning access to data and DNA can be found in the document titled H3Africa Data and Biospecimen Access Committee Guidelines, available in the consortium documents section of the H3Africa website (www. h3africa.com).

\section{Data collection}

The data collected included socio-demographic determinants of health, behavioral risk factors, and metabolic risk factors of CVDs, and the variables relevant to this paper are described briefly below.

\section{Outcome variable: Common CIMT}

The outcome variable was the mean CIMT thickness in micrometers $(\mu \mathrm{m})$ of the far walls of both the left and right CCA. Images were taken with a linear-array 12L-RS transducer using GE Healthcare B-mode LOGIQ e ultrasound machine (GE, Healthcare, CT, USA). To measure the right CCA, the participant was asked to lie down in a supine position with a pillow underneath the neck for slight extension, head turned towards the left at a 45-degree angle and gel applied to the exposed neck area. Using the two sternocleidomastoid muscles as landmarks, the exposed area was scanned along the longitudinal plane until the CCA was found. The operator then identified a continuous one-centimeter segment $(10 \mathrm{~mm})$ of the CCA far wall after which the image was frozen. The operator then placed a cursor between two points ( $10 \mathrm{~mm}$ apart) on this identified segment of the far wall with the proximal starting point one centimeter from the bulb of the CCA. The inbuilt software in the ultrasound equipment then automatically detected the intima-lumen and the media-adventitia interfaced and calculated the minimum, maximum and mean common CIMT in $\mathrm{mm}$ to two decimal places. To measure the left carotid, the participant's head was turned to the opposite side, and the process was repeated (24). We measured one site or angle of the CCA instead of multiple carotid sites or angles, because 
it was easier to measure and was equally reliable at enabling its widespread use at all study sites and aligned to real life setting measurements (31-33). To ensure reproducibility and reduce CIMT measurement variability, masked repeated measurements of the 15 volunteers were conducted by each trainee and the lead trainer. The coefficient of variation between and within trainees was calculated and maintained at $<2 \%$. Subsequently, the same settings and calibrations of the ultrasound equipment were used at each site for data collection.

\section{Exposure variables: Adiposity phenotypes}

The different adiposity phenotypes included in this analysis were body mass index, hip circumference, waist circumference, visceral adipose tissue and subcutaneous adipose tissue. BMI: Standing height to the nearest $0.1 \mathrm{~mm}$ and weight to the nearest $0.1 \mathrm{~kg}$ of each participant were measured without shoes and in light clothes using a Harpenden stadiometer (Holtain, Crymych, Wales) fixed to the wall and a digital calibrated weighing scale (SECA, Hamburg, Germany) respectively. The BMI was subsequently calculated as weight in $\mathrm{kg} /$ height in $\mathrm{m}^{2}$.

\section{Waist circumference (WC) and hip circumference (HC)}

WC was measured using a stretch-resistant tape measure (SECA, Hamburg, Germany). Participants were asked to wear only light or tight-fitting clothing, with the outer clothing removed to enable the tape to be positioned correctly. The participant breathed normally and stood straight with arms slightly abducted when the tape was placed horizontally around the narrowest part of the torso, about halfway between the iliac crest and the lowest rib. Measurements of the WC were taken at the end of a normal expiration without the compression of the tape. The WC was recorded to the nearest $0.1 \mathrm{~cm}$. The HC was measured by placing the tape around the most protruding part of the buttocks, ensuring that the zero mark was to the participant's side. The HC was measured to the nearest $0.1 \mathrm{~cm}$.

\section{Visceral (VAT) and subcutaneous (SCAT) adipose tissues}

These were measured using a B-mode. LOGIQ e ultrasound machine (GE, Healthcare, CT, USA) with a $2.5 \mathrm{MHz} 3 \mathrm{C}-\mathrm{RS}$ curved array transducer. A depth of $15 \mathrm{~cm}$ and $9 \mathrm{~cm}$ were used for VAT (medial) and SCAT measurements, respectively. For both measurements, participants were in the supine position, gel applied to the lower abdomen and the probe positioned with minimal compression on the midline at a level midway between the lower costal margin and the iliac crest with appropriate adjustments in the gain settings. The xiphisternum and umbilicus were used as a guide for accurate positioning. For VAT imaging, the transducer was held horizontal and the spine visualised in the 
horizontal position with the vertebra in the centre of the image. The participant was then asked to breathe quietly, and the measurement was taken at the end of the exhalation. To calculate the amount of VAT, the paused ultrasound image was brought up onto the screen. The first cursor was placed anterior to the spine (on the fat pad if visible), and the second cursor on the thin peritoneal layer beneath the anterior rectus abdominal muscles. Care was taken to ensure that the measurement was perpendicular to the surface of the lumbar vertebra and taken between the peritoneum and the spine where there is a clear space between the vertebra and the aorta. The measurement was repeated by producing a second image, and the results recorded in $\mathrm{cm}$ to two decimal places. An immediate quality check was done to ensure that the spine, abdominal aorta and rectus abdominal muscle were visualized on the image $(34,35)$.

For the measurement of SCAT (transverse), the ultrasound probe was rotated through 90 degrees and the depth setting adjusted to $9 \mathrm{~cm}$. The rectus abdominus muscles were visualized, with care taken to ensure that both muscles were symmetrical in the image, and that the linea alba was centrally located, the gain adjusted accordingly, and the image captured. To calculate the SCAT measurement, the distance between the skin and the outer edge of the linea alba was measured on the screen, as described above. The measurement was then taken from a second image, and the results recorded similarly, in $\mathrm{cm}$ to two decimal places. The reliability of these ultrasound measurements in estimating adiposity has previously been validated in a black South African population (35).

\section{Covariates}

Selected covariates were those that contribute to the development of CVDs and can confound the association between adiposity phenotypes and CIMT. These included age, level of education (highest level of education obtained), household socio-economic status (estimated by calculations based on household assets, according to the practice implemented by the Demographic and Health Surveys (DHS) Program) (24), alcohol consumption, smoking and physical inactivity assessed by measuring moderate-to-vigorous physical activity (MVPA). Physical inactivity was defined as MVPA $<150$ mins/week. Other covariates included systolic blood pressure, glucose, total cholesterol, HDL-C, LDL-C and HIV infection and ART use. Post-menopausal status in women was defined as having no period within the past 12 months. Details of how these variables were measured have been reported previously (24). 


\section{Data analysis}

All data analyses were conducted with STATA version 14.1 (College Station, Texas, USA). We computed an average of the mean right and left far wall common CIMT thickness in micrometers $(\mu \mathrm{m})$ as the main outcome variable. Continuous data were normally distributed and were summarized as means with standard deviations $( \pm S D)$ and categorical data presented as absolute counts with corresponding percentages (\%). Differences in mean ( \pm SD) levels of the various adiposity phenotypes between women and men within the sites were determined using Student t-tests. We determined the association between each adiposity phenotype and CIMT using an inverse-variance weighted fixedeffect individual participant data meta-analysis (IPD-MA) using the "ipdmetan" package in STATA. This approach offered standardisation of analyses across study sites while taking into account potential clustering and heterogeneity of the different study populations (36). We were thus able to compare the magnitude of the effect of each adiposity phenotype on CIMT across the various study sites. In these analyses, we obtained a test of the overall effect of each adiposity measure on CIMT in the total sample. We also obtained betweenstudy variance as a percentage of the total variance between study populations $\left(1^{2}\right)$, giving us an idea of the extent of heterogeneity. Forest plots are plotted to display the effect measure and the percentage weight of the various study populations.

Where heterogeneity is significant as demonstrated by a higher $\% l^{2}$, subgroup analyses were conducted for each site. In these sub-group analyses, the association of adiposity phenotypes with CIMT in each separate site was assessed using adjusted multiple linear regression analyses. These analyses were done in multiple sequential models. In the first step, we initially determined the independent association between each adiposity phenotype and CIMT. We then adjusted for covariates in a sequential manner. In Model 1, we adjusted for age, level of education, and household SES; Model 2 included variables from Model 1 plus additional adjustments for smoking, alcohol consumption, and MVPA. Model 3 was based on Model 2 plus each of the other body adiposity phenotypes. Adiposity phenotypes that had a variance inflation factor (VIF) >5 were dropped from Model 3. Thus, BMI, WC and $\mathrm{HC}$ were strongly correlated and were therefore not included in Model 3, whereas VAT and SCAT were included. Model 4 included Model 3 plus systolic blood pressure, glucose, HDL-C, LDL-C, total cholesterol and HIV infection. In women, menopausal status was also adjusted for in Model 4.

Measures of associations are reported as standardised beta $(\beta)$ coefficients denoting differences in mean CIMT in $\mu \mathrm{m}$ caused by a unit increase in the adiposity phenotypes. Statistical significance was set at two- sided, $\mathrm{p}<0.05$. 


\section{Results}

Data were available for 10,863 participants, but 1,341 had no CIMT data which included all women from Soweto. In addition, 512 participants had missing data from the other variables. Therefore, we conducted complete case analyses of 9,010 participants from six sites in four countries.

\section{Descriptive data}

The basic characteristics of the AWI-Gen study participants stratified by study site and sex are presented in Table 1. For the combined data from all sites, women had a mean age of $50.1 \pm 6$ years compared to $49.8 \pm 9$ years for men. In all sites, men had a higher mean household SES compared to women and similar observations were made for educational attainment (Table 1). Men showed a higher prevalence of both current smoking and alcohol consumption than women at all sites. Physical activity levels varied across sites, but women were more likely to be physically active. The prevalence of obesity was higher in women than men at all sites except Nanoro, Burkina Faso. Both women and men from Navrongo had the highest mean common CIMT followed by Nanoro, Dikgale, Agincourt and Soweto (men only) with the lowest levels in both sexes being observed in Nairobi (Table 1).

The mean levels of the various adiposity phenotypes by site and gender are summarized in Table 2. Gender differences ( $p<0.001$ for all) were noted for all adiposity measures at all sites, with the exception of VAT and SCAT at Nanoro. 


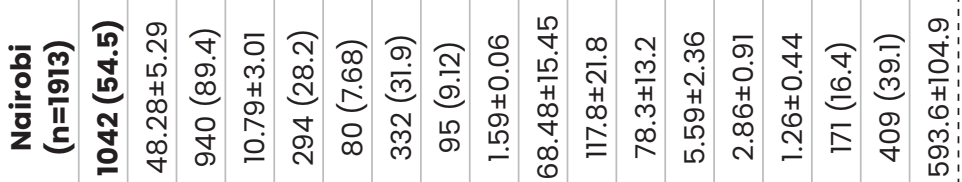

象宽

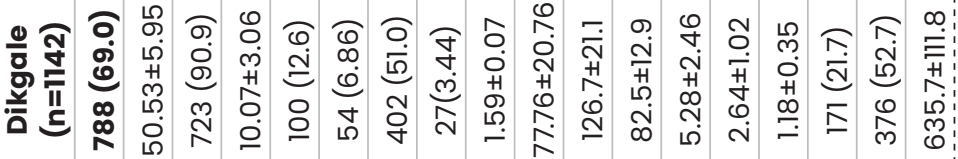

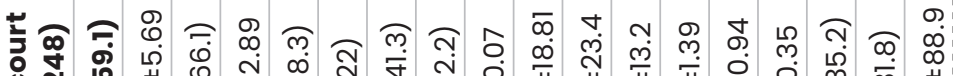

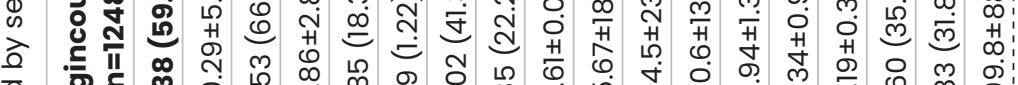

司

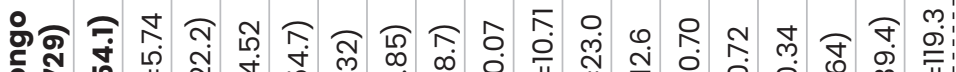

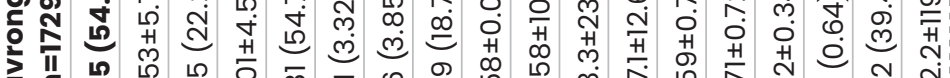

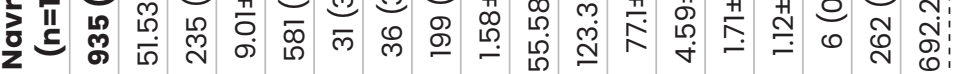

ह

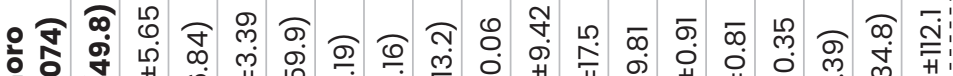

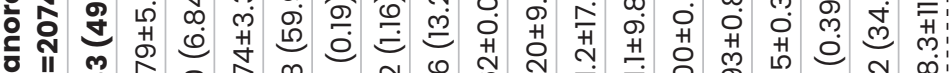

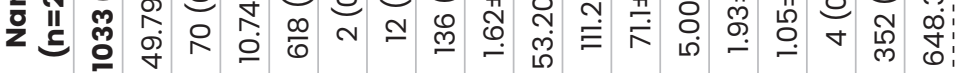

$\frac{. \overline{0}}{\overline{\frac{1}{0}}}$

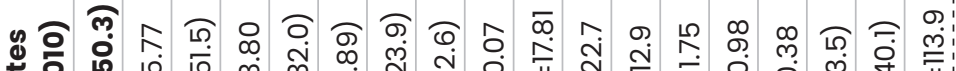

总家

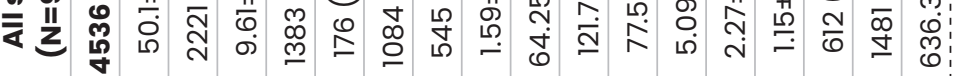

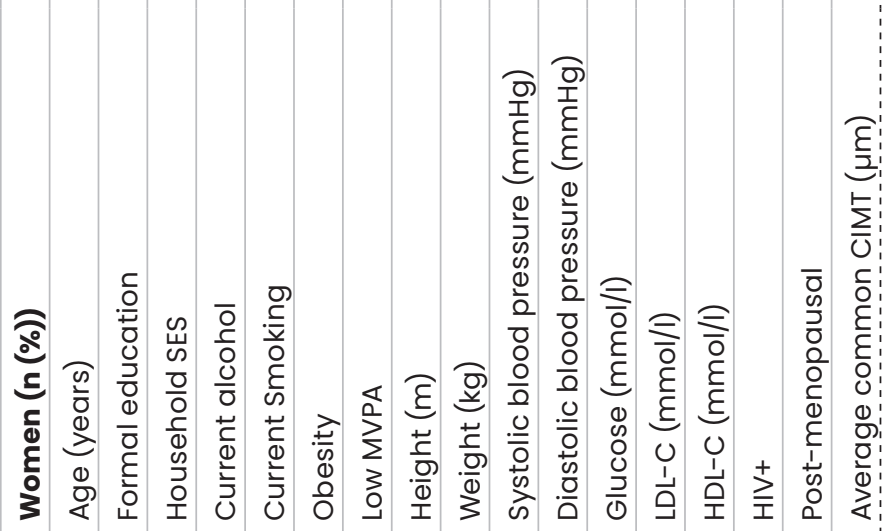


产 $\frac{1}{0}$ ठ̀

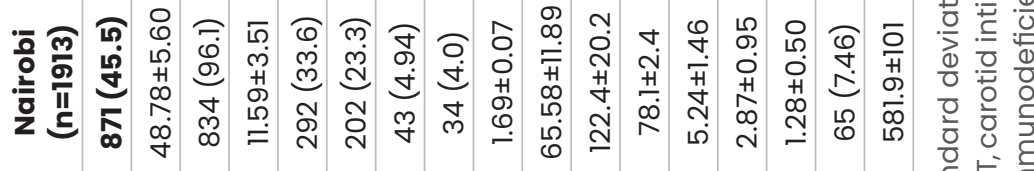

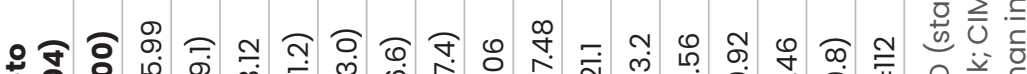

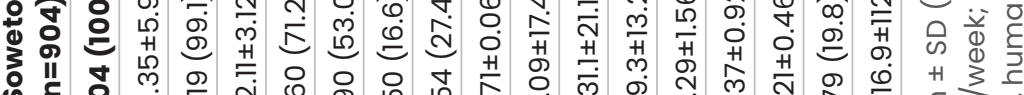

仓ீ

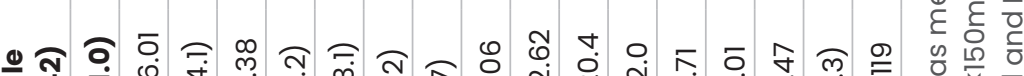

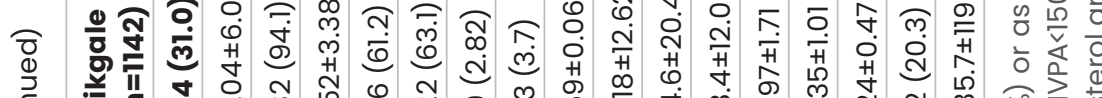

든

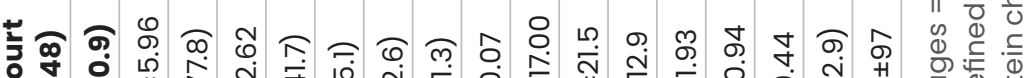

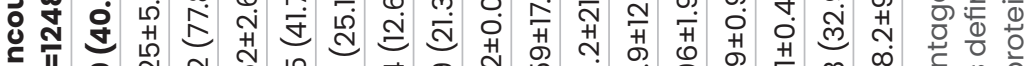

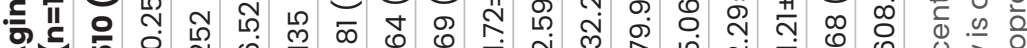

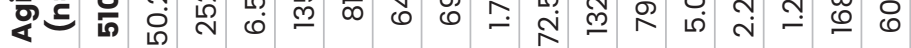

니을

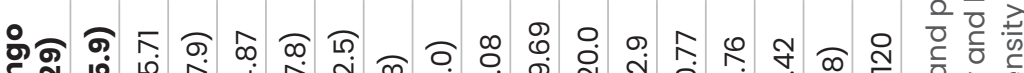

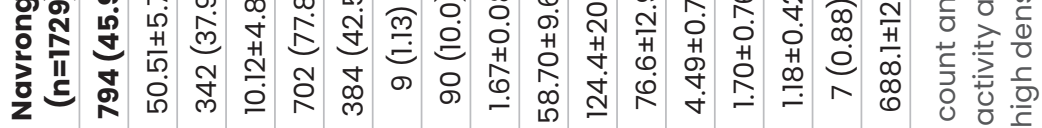

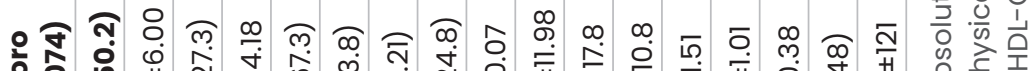

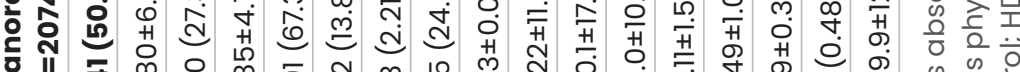

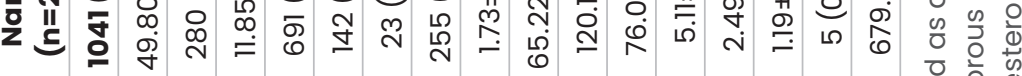

ต ก

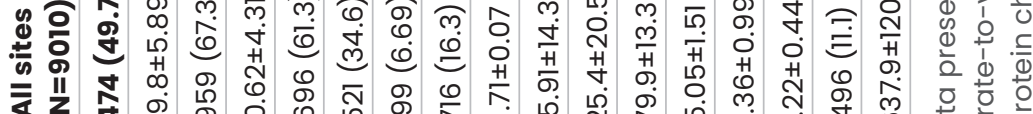

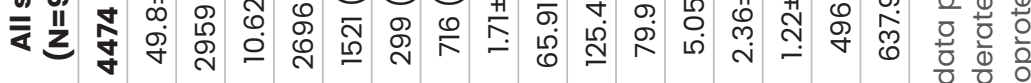

$$
\frac{1}{(1)}
$$

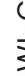

$\sum_{\substack{1 \\ \hline}}^{1}$

4

$\frac{0}{i=0}$

$\frac{1}{0}$
$\frac{0}{0}$
$\frac{0}{0}$
$\frac{0}{0}$
$\frac{1}{0}$

$\frac{0}{9}$

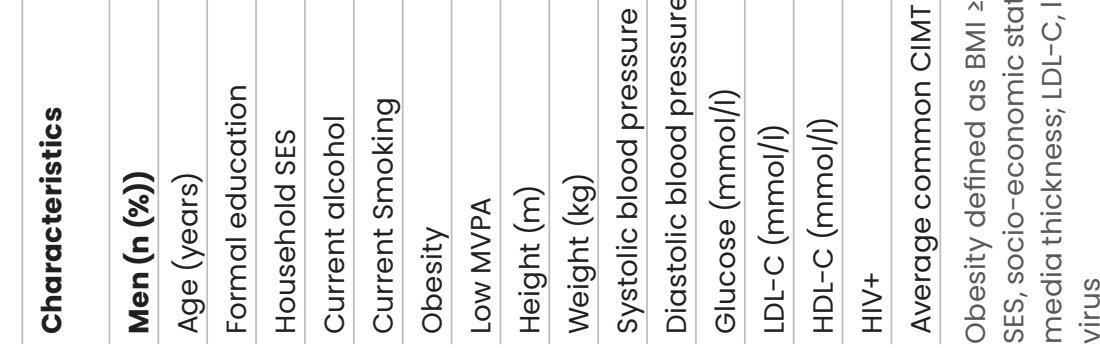




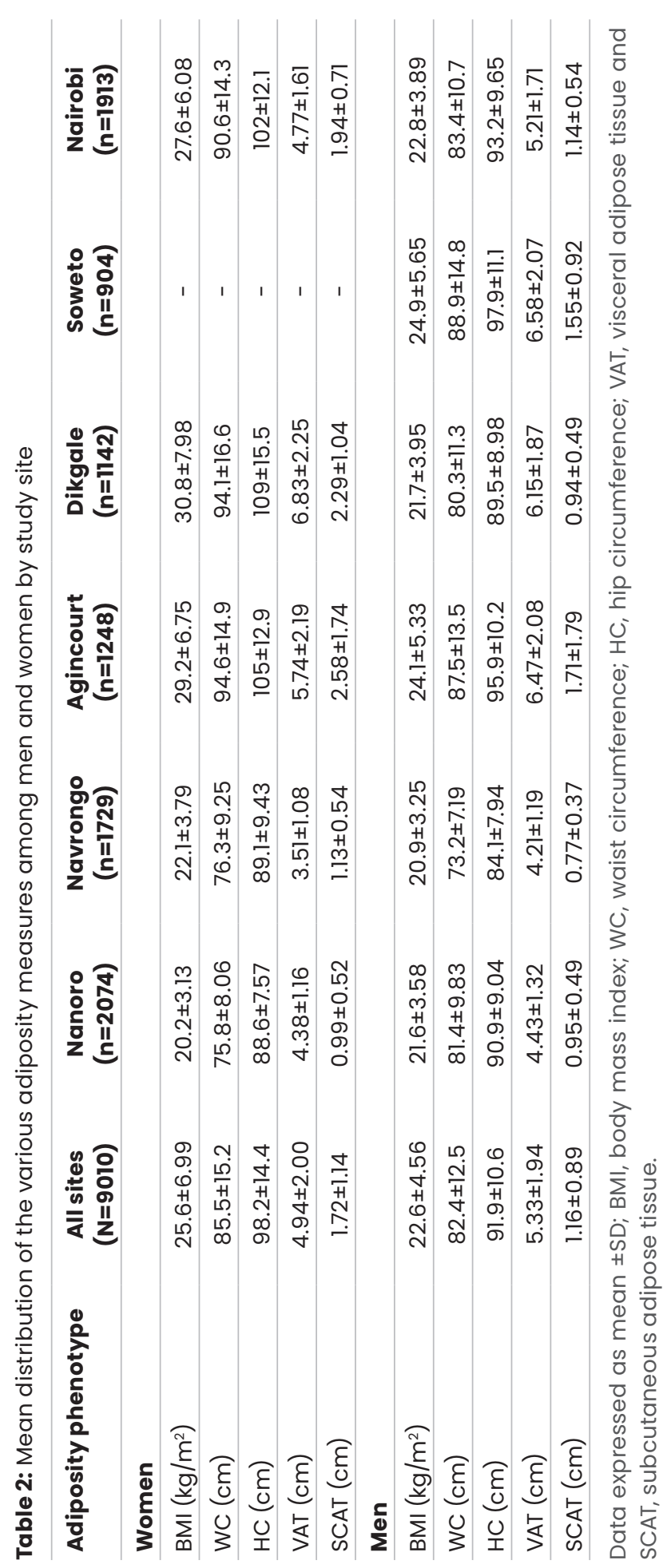




\section{Relationship between adiposity phenotypes and CIMT}

Figures 1-5 present the forest plots showing the site-specific and pooled IPD-MA of the association of each adiposity phenotype with CIMT. There was an overall positive effect of BMI on CIMT in the pooled analyses and in all sites, but the effect in Navrongo was not statistically significant (Figure 1). The IPD-MA for WC (Figure 2), HC (Figure 3) and SCAT (Figure 5) also gave significant positive pooled effects. In Nanoro, VAT had an inverse association with CIMT while the pooled effect was positive (Figure 4). The pooled IPD-MA demonstrated that BMI had the highest effect measure followed by SCAT, VAT, WC and HC. These analyses demonstrated between-site heterogeneity which shows that the fixed-effect assumption (that the effect is the same at each site) is incorrect.

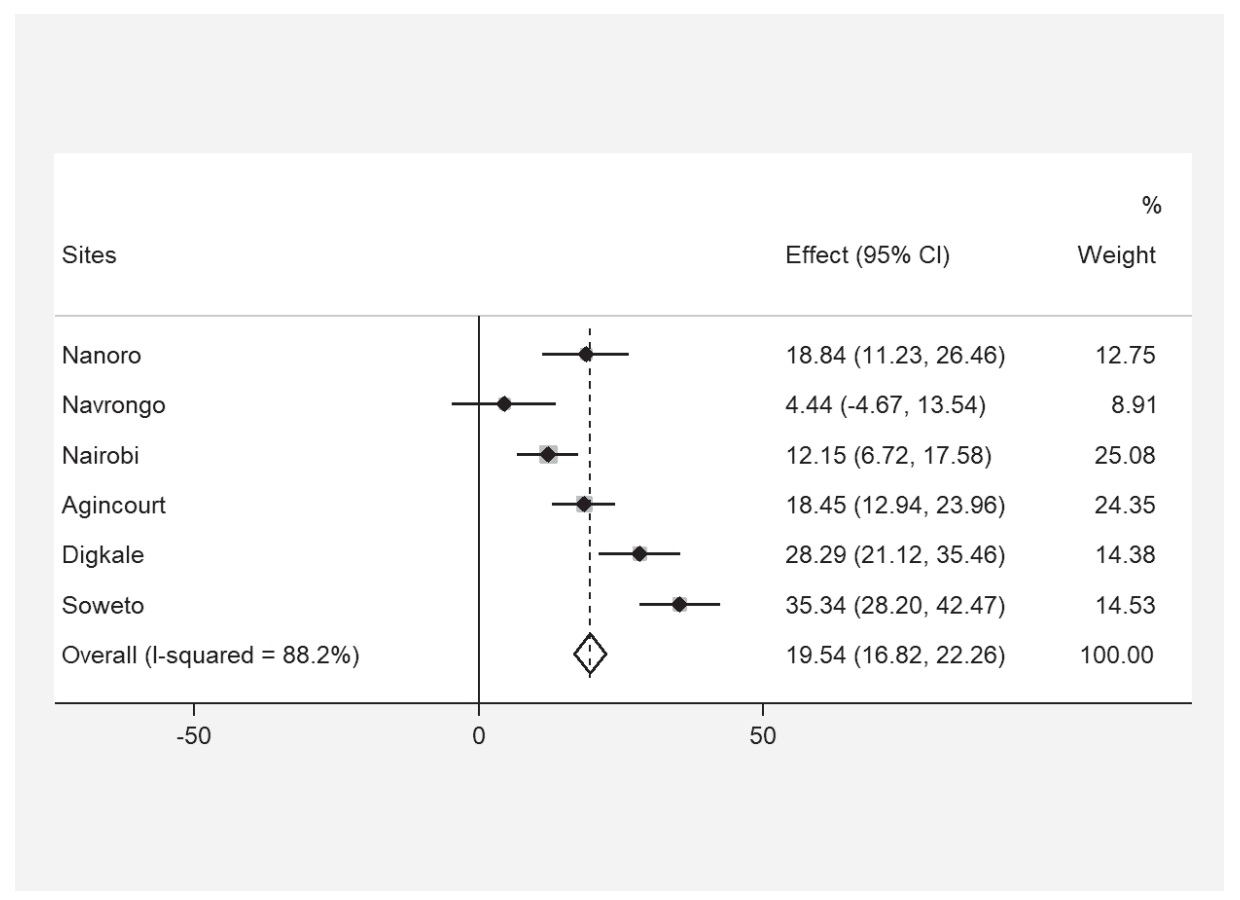

Figure 1: Forest plot displaying an inverse-variance weighted fixed-effect individual participant data meta-analysis of the effect of $\mathrm{BMI}\left(\mathrm{kg} / \mathrm{m}^{2}\right)$ on common carotid intima-media thickness $(\mu \mathrm{m})$; the effect size (beta, $\beta$ ) and $95 \%$ Cls are presented by the symbol and the bars respectively; the big diamond represents the overall effect of BMI in the poled data and the grey squares represent the $\%$ weight of each study site. 


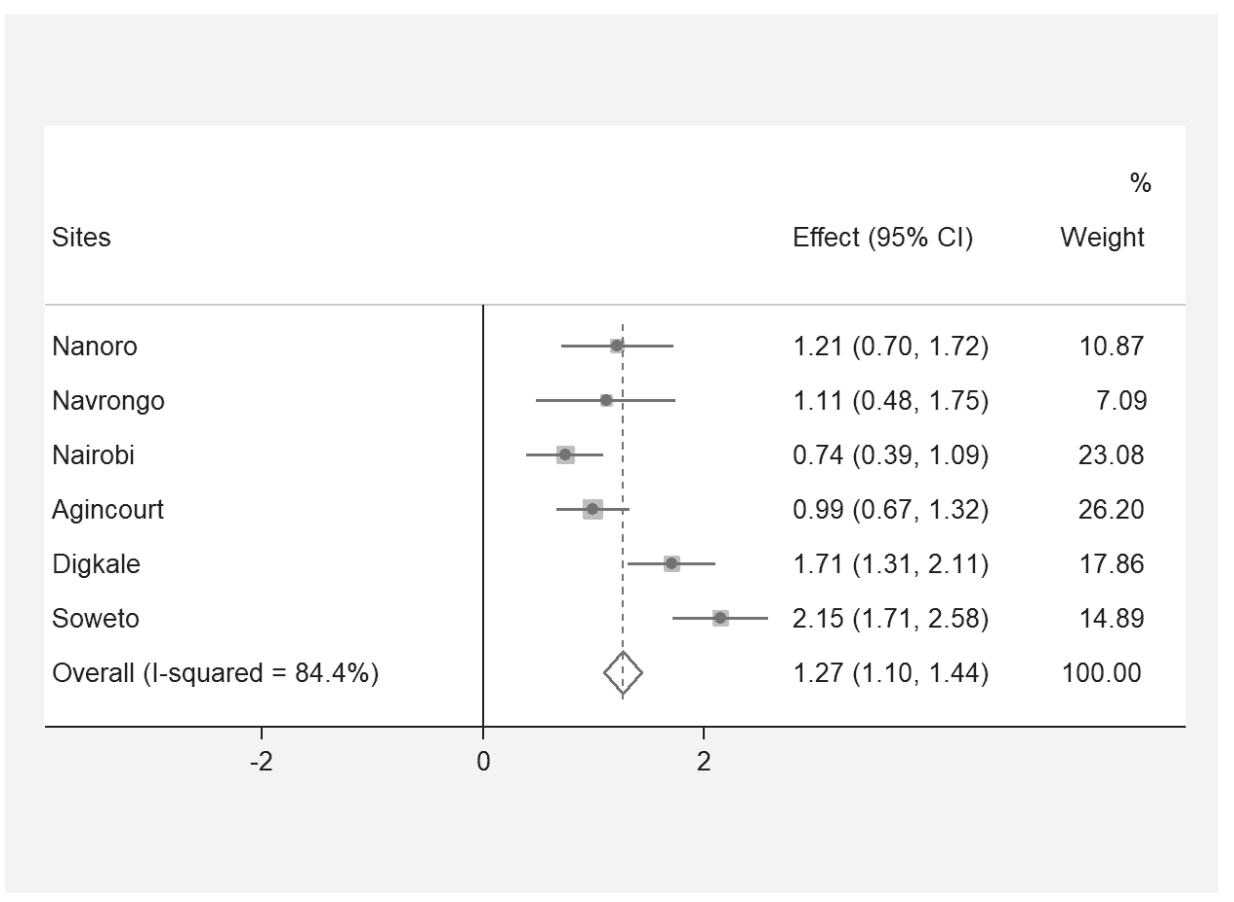

Figure 2: Forest plot displaying an inverse-variance weighted fixed-effect individual participant data meta-analysis of the effect waist circumference $(\mathrm{cm})$ on common carotid intima-media thickness in $\mu \mathrm{m}$; the effect size (beta, $\beta$ ) and $95 \% \mathrm{Cls}$ are presented by the symbol and the bars respectively; the big diamond represents the overall effect of waist circumference in the poled data and the grey squares represent the \% weight of each study site. 


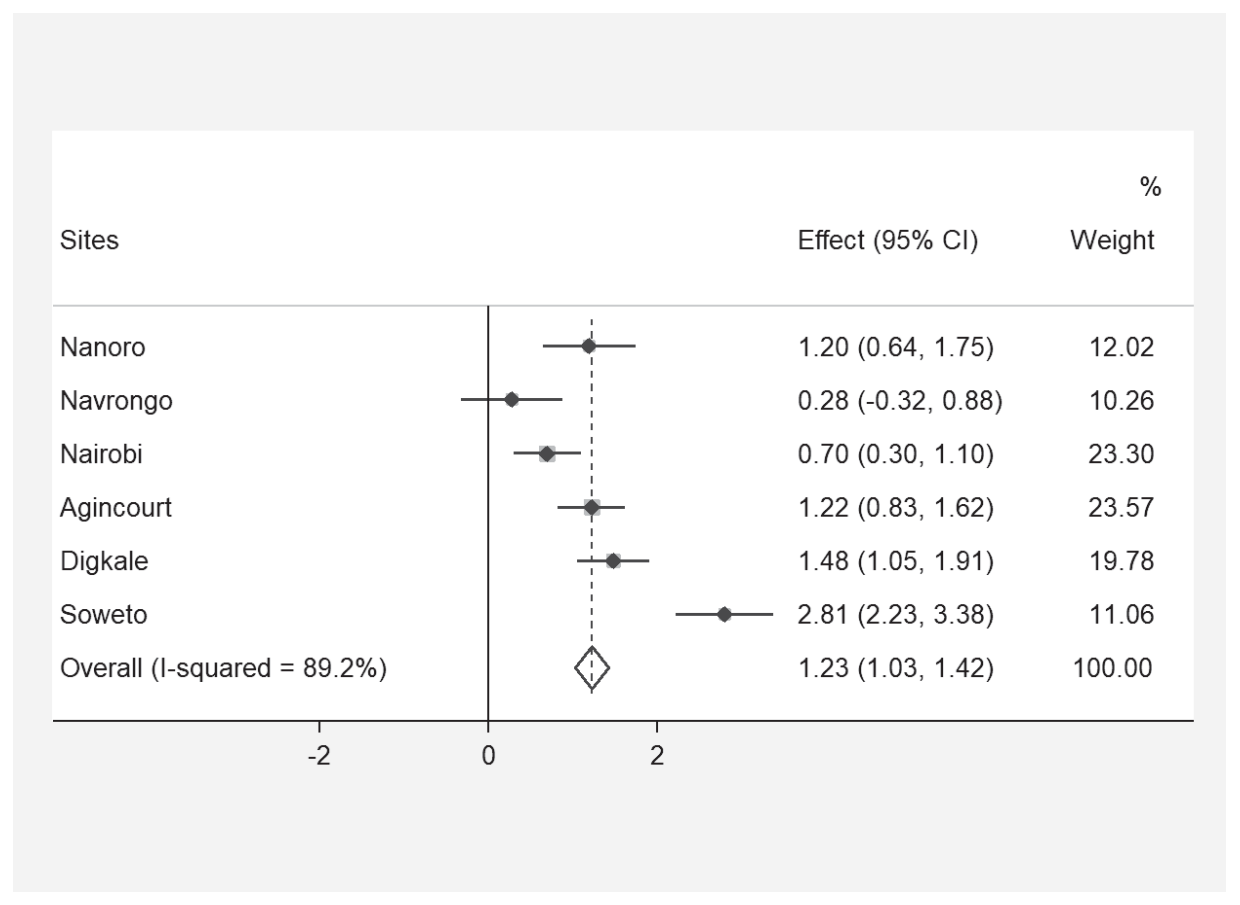

Figure 3: Forest plot displaying an inverse-variance weighted fixed-effect individual participant data meta-analysis of the effect hip circumference $(\mathrm{cm})$ on common carotid intima-media thickness in $\mu$ m; the effect size (beta, $\beta$ ) and $95 \%$ Cls are presented by the symbol and the bars respectively; the big diamond represents the overall effect of hip circumference in the poled data and the grey squares represent the \% weight of each study site. 


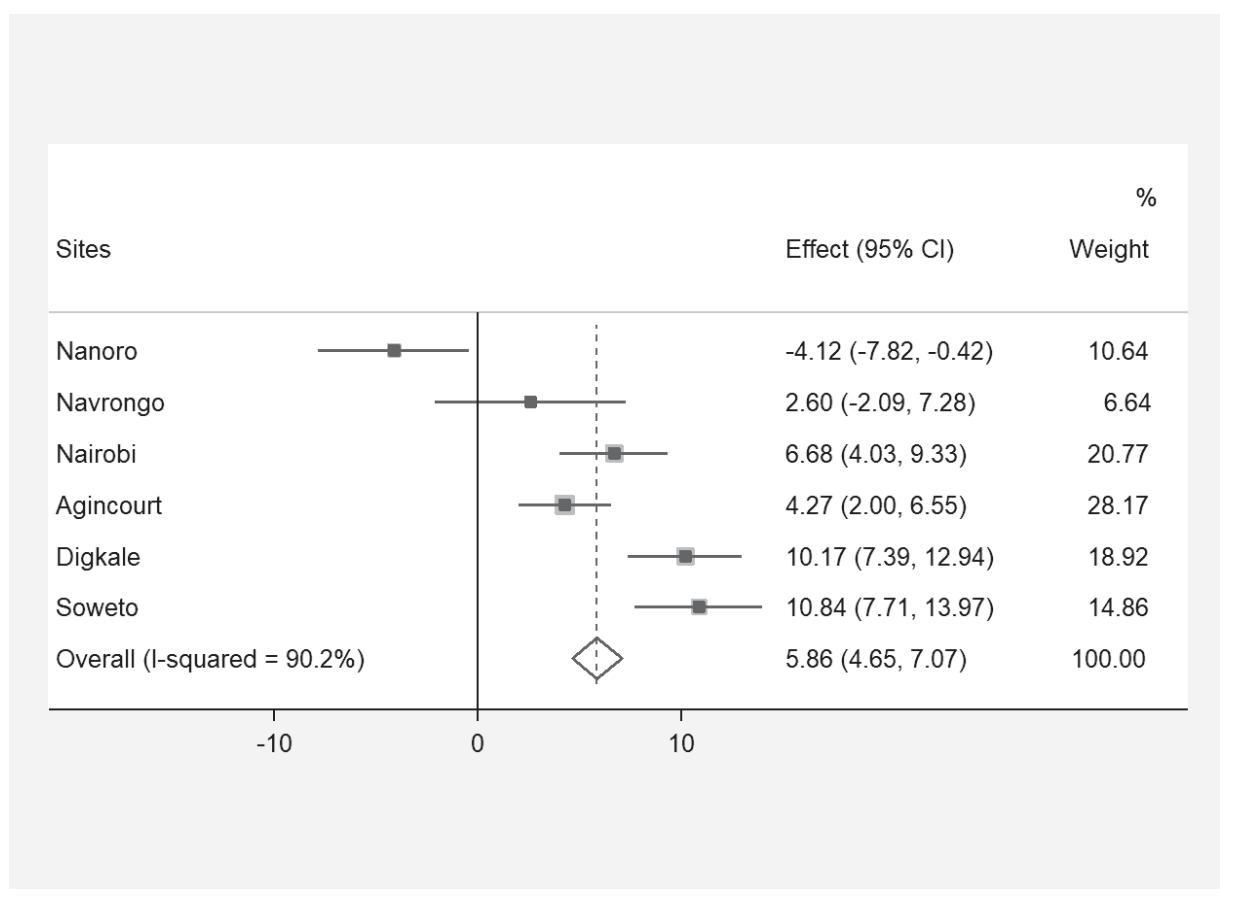

Figure 4: Forest plot displaying an inverse-variance weighted fixed-effect individual participant data meta-analysis of the effect visceral adipose tissue $(\mathrm{cm})$ on common carotid intima-media thickness in $\mu \mathrm{m}$; the effect size (beta, $\beta$ ) and $95 \% \mathrm{Cls}$ are presented by the symbol and the bars respectively; the big diamond represents the overall effect of visceral adipose tissue in the pooled data and the grey squares represent the $\%$ weight of each study site. 


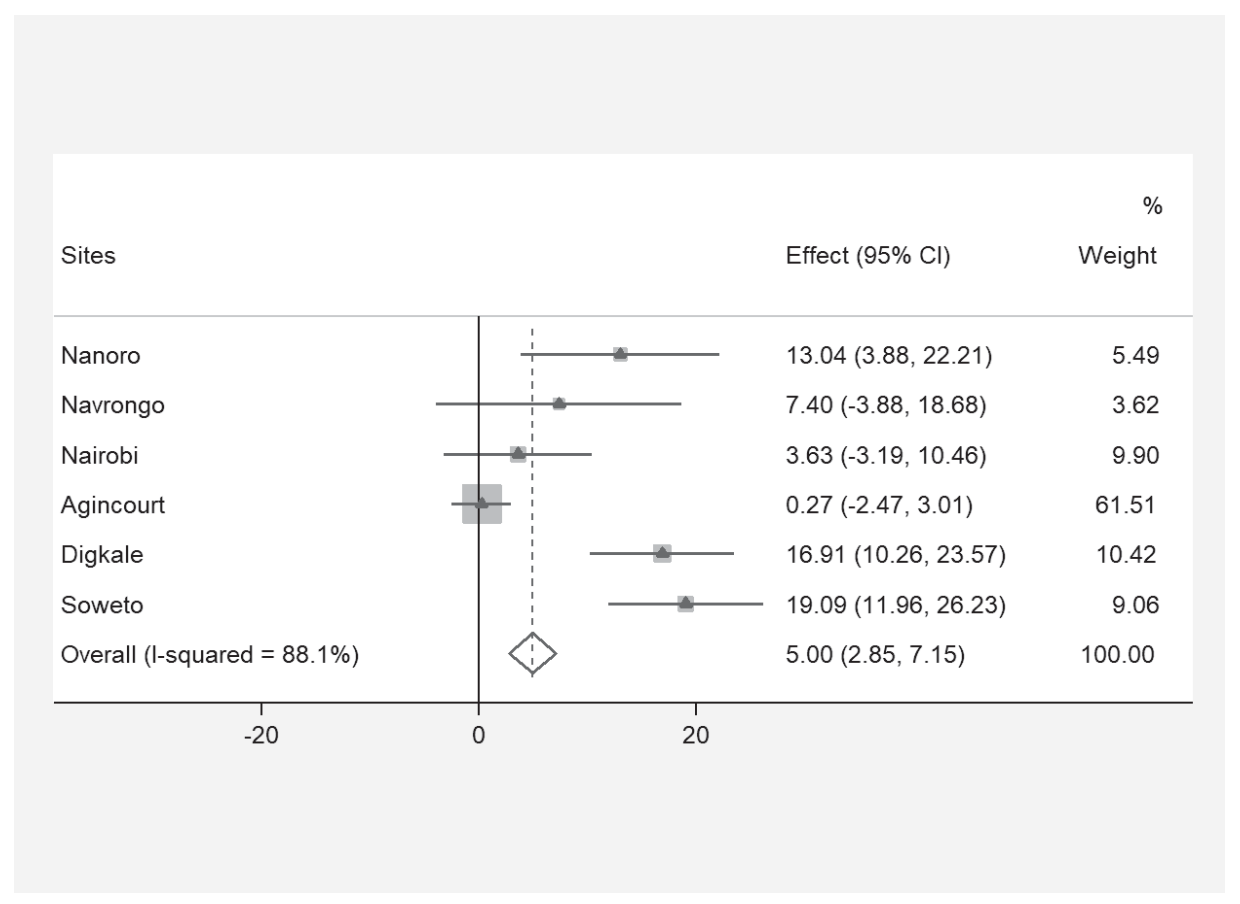

Figure 5: Forest plot displaying an inverse-variance weighted fixed-effect individual participant data meta-analysis of the effect subcutaneous adipose tissue $(\mathrm{cm})$ on common carotid intima-media thickness in $\mu \mathrm{m}$; the effect size (beta, $\beta$ ) and $95 \% \mathrm{Cls}$ are presented by the symbol and the bars respectively; the big diamond represents the overall effect of subcutaneous adipose tissue in the pooled data and the grey squares represent the \% weight of each study site.

The variability in the effect-size estimates is therefore due to between-site differences rather than sampling variation. We therefore conducted separate analyses for women and men in each site and reported these results in supplementary Tables S1 and S2. The summary measures of heterogeneity and the test of overall effect are presented in supplementary Table S3.

The strength of the associations between each adiposity measures and CIMT stratified by sex varied across the sites, however the direction of the significant associations were the same for all the adiposity phenotypes except VAT and SCAT. VAT was negatively associated with CIMT at Nanoro in both women and men and negatively associated in men at the Navrongo site while SCAT was inversely associated with CIMT among women at all sites. Adjusting for covariates in the models that included all women or all men did tend to affect the strength of the associations, but it did not change the direction of the relationship or render the association significant or non-significant when compared to the initial unadjusted model. The strength of association of each 
anthropometry measure with CIMT was higher in men than in women at all the study sites (supplementary Tables S1 and S2).

\section{Discussion}

In this study, we examined the association between various adiposity phenotypes and common CIMT in African populations from Burkina Faso, Ghana, Kenya and South Africa. The selected age group of 40-60 years has been identified by World Health Organization to be at risk of "premature death" from CVDs (5), indicating significant public health implications of the current findings. We standardized our estimates to enable us to compare the magnitude of the effect of the various adiposity phenotypes on CIMT by sex and the various study sites. We observed that all adiposity phenotypes were positively associated with an increase in CIMT in the pooled analyses. The associations were stronger in men than in women except for SCAT where the association was stronger in women than in men. We further observed that, after adjusting for possible confounding variables, BMI had the strongest association with CIMT followed by VAT, SCAT, WC and HC.

Our finding that various adiposity phenotypes were associated with greater CIMT in both women and men expands the evidence on this matter since it has been previously reported in European, American, African- American and Asian populations (17-19, 37, 38). In our study, BMI showed a stronger association with CIMT compared to the other measures of adiposity. This is contrary to many studies which have shown that central adiposity, particularly VAT, is a stronger determinant of CVD risk than BMI $(11,39)$. However, none of these studies included data collected from African populations resident in sub-Saharan Africa. A study from Malawi previously reported a stronger association of general obesity with hyperglycemia and elevated blood pressure over that of central obesity (40). In support of the results produced in our investigation, a study conducted on HIV-positive participants from Uganda demonstrated that BMI, but not waist circumference, correlated positively with CIMT (41). The attenuated effect of VAT and markers of central adiposity on CIMT in sub-Saharan African populations may be related to the lower level of VAT observed in these populations when compared to other ethnic groups $(12,13,42,43)$.

We observed that there was a positive effect of BMI on CIMT in the two West African sites which had the lowest levels of BMI but the highest CIMT levels. Biologically, obesity is known to be associated with CVD risk and our finding supports this observation. However, we noticed the magnitude of the effect of $\mathrm{BMI}$ on atherosclerosis was smaller in these two sites compared to the other sites that had higher obesity levels. The high CIMT in West Africa must therefore 
be due to other variables that were not captured in this study as many factors are known to influence CIMT other than BMI.

The association between various measures of body adiposity and CIMT may be mediated by other CVD risk factors such as high serum cholesterol and blood pressure levels. However, after adjusting for these risk factors in multivariable regression models, the association between adiposity phenotype and CIMT was attenuated, but still remained significant. Thus, other factors must be mediating the effect of adiposity on CIMT and previous studies suggest that these may include inflammation, insulin resistance and endothelial dysfunction (44).

In our study, SCAT was positively associated with CIMT in men but inversely associated with CIMT in women, and these gender-specific associations were consistently observed at each study site. The inverse association of SCAT with CIMT observed in women is supported by other studies that have observed that SCAT may be protective against several diseases including atherosclerosis. Narumi et al (2009), who assessed atherosclerosis using the calcium score of the whole aorta, demonstrated an inverse association between SCAT and atherosclerosis in an apparently healthy Japanese population (45). Bouchi et al (2015) further observed a protective effect of SCAT on CIMT among Japanese type 2 diabetes patients (46). A study by Glesby et al (2018) in a group of HIVpositive American females showed that higher SCAT levels were associated with a lower risk of carotid artery plaques (47). The protective effect of SCAT may be due to the expression of a less pro-inflammatory adipokine profile compared to visceral fat $(39,48)$ and/or the ability of SCAT to act as a buffer for lipid flux thus reducing triglyceride deposition in visceral or ectopic fat depots (49). Women had higher mean SCAT levels compared to men and this may contribute to the greater protective effect observed in this gender.

In addition, abdominal SCAT is composed of two different compartments termed superficial and deep SCAT, with women having lower levels of deep but higher levels of superficial SCAT than men (50). Furthermore, a study has shown that deep SCAT is positively associated with the Framingham risk score in multivariable regression models in men but not women (51). It is uncertain whether these sex differences in abdominal SCAT compartments and their differential association with cardiovascular risk may explain the opposing effects of SCAT on CIMT observed in men and women. Levels of deep and superficial SCAT were not measured in this study and further analysis of these SCAT compartments and their association with CIMT is required in African populations. Also, the higher 95\% Cls for the coefficients for SCAT and the high standard deviations for the SCAT mean values may imply some level of measurement imprecision, further affecting the outcome. 
We observed that the association of each adiposity phenotype (with the exception of SCAT) with CIMT was stronger in men than women. This is supported by a study from Taiwan which showed that BMI was more strongly associated with CIMT in men than women (52). Studies have shown that sex differences exist in adipocyte function and secretory profiles (53), but whether such differences explain the differential effects of obesity on CIMT in men and women is not known. It is also possible that this sex difference may be due to differences in the component causes for increased CIMT between men and women.

At most of the study sites, VAT was positively associated with CIMT, but in men in Navrongo and both women and men in Nanoro, West Africa, these associations were in the opposite direction. The reason for this is not known. However, VAT levels at these sites were the lowest of all the sites, whilst CIMT was the highest. In addition, the majority of the participants from West Africa were subsistence farmers, whilst this was not the case in the other regions. It is therefore possible that biological, behavioural and socio-demographic differences across the sites that have not been captured in the current study may explain these variations in the association of VAT with CIMT, and require further investigation. However, it must also be noted that some of the site differences may be due to random error or differences in the population selection at the various sites.

\section{Strengths and limitations}

This study contains data on CIMT and adiposity phenotypes from a large population of black African subjects from three sub-continental regions, that is, East, West and South Africa and, to the best of our knowledge, is the largest study conducted on populations residing in SSA. Measurements of exposure and outcome variables and covariates were harmonized across the six study sites. This minimized variability and enabled us to make valid comparisons by sex and site across the study populations. Most large population-based studies use crude markers of adiposity, however, in this study we used ultrasound to assess VAT and SCAT levels. Due to the broad range of adiposity measures used in our analyses, we were able to investigate the contribution of general, central and peripheral adiposity to atherosclerosis in the study population. Furthermore, we adjusted for a wide range of confounding variables including socio-economic status, lifestyle factors and other cardiovascular risk factors, to minimize the effect of residual confounding.

There are some limitations to our study. Due to the cross-sectional study design, it is not possible to infer causality. Even though we included a wide range of possible confounding variables, our observed associations may still be affected by persistent residual confounding due to unmeasured variables that may better explain reported associations. Since most of the risk factors were 
self-reported, there is the risk of recall bias and selective response leading to some biased estimates. The gold-standard methods for measurement of VAT and SCAT include CT and MRI imaging, whereas we used B-mode ultrasound measures. However, this method was the only practical way of measuring VAT, SCAT and CIMT in a large population-based study across multiple sites. Despite significant efforts instituted to reduce inter-sonographer measurement variation, we fully acknowledge that there may be some level of error obtained in the measurements in the field.

\section{Conclusions}

The results from this study suggest that the increasing prevalence of obesity within SSA will likely result in a surge in atherosclerotic cardio- and cerebrovascular events, especially in males, unless addressed through primary prevention of obesity in both rural and urban communities across Africa. The inverse association between VAT and CIMT in West Africa (Burkina Faso and Ghana) requires further investigation.

\section{Acknowledgements}

The AWI-Gen study would not have been possible without the generosity of the participants who spent many hours responding to questionnaires, being measured and having samples taken. We wish to acknowledge the sterling contributions of our field workers, phlebotomists, laboratory scientists, administrators, data personnel and other staff who contributed to the data and sample collections, processing, storage, and shipping. Investigators responsible for the conception and design of the original AWI-Gen study include Michèle Ramsay (PI, Wits), Osman Sankoh (co-PI and former director of INDEPTH network, Accra, Ghana), Alisha Wade, Stephen Tollman and Kathleen Kahn (Agincourt), Marianne Alberts (Dikgale), Catherine Kyobutungi (Nairobi), Halidou Tinto (Nanoro), Abraham Oduro (Navrongo), Shane Norris (Soweto), and Scott Hazelhurst, Nigel Crowther, Himla Soodyall and Zane Lombard (Wits).

We would like to acknowledge each of the following investigators from the various sites for the significant contributions made to this research: Agincourt, South Africa - Frances Xavier Gómez-Olivé Casas, Ryan Wagner and Kathleen Kahn; Dikgale, South Africa - Ian Cook and Sam Ntuli; Nairobi, Kenya - Christopher Khayeka-Wandabwa, Tilahun N. Haregu, Gershim Asiki and Stella Muthuri; Nanoro, Burkina Faso - Toussaint Rouamba. Navrongo, Ghana - Eric Fato, Immaculate Anati, Cornelius Debpuur and Lucas Amenga-Etego; Soweto, South Africa - Nomses Baloyi, Juliana Kagura, Richard Munthali and Yusuf Guman. We also appreciate the work of Scott Hazelhurst and Freedom 
Mokumana at the Wits AWI-Gen Collaborative Centre in Johannesburg for all the quality control checks to ensure that we obtained good quality data.

\section{Funding Information}

The AWI-Gen Collaborative Centre is funded by the National Human Genome Research Institute (NHGRI), Office of the Director (OD), Eunice Kennedy Shriver National Institute Of Child Health \& Human Development (NICHD), the National Institute of Environmental Health Sciences (NIEHS), the Office of AIDS research (OAR) and the National Institute of Diabetes and Digestive and Kidney Diseases (NIDDK), of the National Institutes of Health (NIH) under award number U54HG006938 and its supplements, as part of the H3Africa Consortium. Additional funding was leveraged from the Department of Science and Technology, South Africa (SA DST), award number DST/CON 0056/2014. EAN is supported by a grant from the Global Health PhD Support Program of the University Medical Center Utrecht (UMCU), Utrecht University, The Netherlands and the Navrongo Health Research Centre (NHRC), Ghana. This paper describes the views of the authors and does not necessarily represent the official views of the National Institutes of Health (USA) or the SA DST.

\section{Competing Interests}

The authors have no competing interest to declare.

\section{Author Contribution}

EAN, MLB, KKG and NJC conceived the paper. EAN, ARO, GA, CCS, LKM, FM, PRB, SFM, ANW, ARO, CK, SAN, SMT, HT, MR and NJC contributed to data collection and data quality control in the $\mathrm{H} 3 \mathrm{Africa}$ AWI-Gen study. EAN conducted the analyses and wrote the first draft with contributions from NJC, MLB and KKG. All authors critically reviewed and approved the final manuscript prior to submission. 


\section{References}

1. Roth GA, Johnson C, Abajobir A, Abd-Allah F, Abera SF, Abyu G, et al. Global, regional, and national burden of cardiovascular diseases for 10 causes, 1990-2015. J Am Coll Cardiol. 2017; 70(1): 1-25. DOI: https://doi.org/10.1016/j.jacc.2017.04.052

2. NCD Risk Factor Collaboration (NCD-RisC) - Africa Working Group. Trends in obesity and diabetes across Africa from 1980-2014: An analysis of pooled population-based studies. Int J Epidemiol. 2017; 46(5): 1421-32.

3. Ramsay M, Crowther NJ, Agongo G, Ali SA, Asiki G, Boua RP, et al. Regional and sexspecific variation in BMl distribution in four sub-Saharan African countries: The H3Africa AWI-Gen study. Glob Health Action. 2018; 11(sup2): 1556561. DOI: https://doi. org/10.1080/16549716.2018.1556561

4. Yusuf S, Reddy S, Ôunpuu S, Anand S. Global burden of cardiovascular diseases part I: General considerations, the epidemiologic transition, risk factors, and impact of urbanization. Circulation. 2001; 104: 2746-53. DOI: https://doi.org/10.1161/hc4601.099487

5. GBD 2015 Risk Factors Collaborators. Global, regional, and national comparative risk assessment of 79 behavioural, environmental and occupational, and metabolic risks or clusters of risks, 1990-2015: A systematic analysis for the Global Burden of Disease Study 2015. Lancet. 2016; 388(10053): 1659-724. DOI: https://doi.org/10.1016/ S0140-6736(16)31679-8

6. NCD Risk Factor Collaboration (NCD-RisC). Rising rural body-mass index is the main driver of the global obesity epidemic in adults. Nature. 2019; 569(7755): 260-4. DOI: https://doi.org/10.1038/ s41586-019-1171-x

7. Libby P. Inflammation and atherosclerosis. Circulation. 2002; 105(9): 1135-43. DOI: https://doi. org/10.1161/hc0902.104353

8. Kinlen D, Cody D, O'Shea D. Complications of obesity. QJM. 2018; 111(7): 437-43. DOI: https://doi. org/10.1093/qjmed/hcx152

9. Wang $H$, Chen $Y E$, Eitzman DT. Imaging body fat: Techniques and cardiometabolic implications. Arterioscler Thromb Vasc Biol. 2014; 34(10): 2217-23. DOI: https://doi. org/10.1161/ATVBAHA.114.303036

10. Cornier MA, Despres JP, Davis N, Grossniklaus DA, Klein S, Lamarche B, et al. Assessing adiposity: A scientific statement from the American Heart Association. Circulation. 2011; 124(18): 1996-2019. DOI: https://doi.org/10.1161/CIR.0b013e318233bc6a

11. Lee MJ, Wu Y, Fried SK. Adipose tissue heterogeneity: implication of depot differences in adipose tissue for obesity complications. Mol Aspects Med. 2013; 34(1): 1-11. DOI: https://doi.org/10.1016/j. mam.2012.10.001

12. Goedecke JH, Levitt NS, Lambert EV, Utzschneider KM, Faulenbach MV, Dave JA, et al. Differential effects of abdominal adipose tissue distribution on insulin sensitivity in black and white South African women. Obesity. 2009; 17(8): 1506-12. DOI: https://doi. org/10.1038/ oby.2009.73 
13. Sumner AE, Micklesfield LK, Ricks M, Tambay AV, Avila NA, Thomas F, et al. Waist circumference, BMI, and visceral adipose tissue in white women and women of African descent. Obesity. 2011; 19(3): 671-4. DOI: https://doi.org/10.1038/oby.2010.201

14. Mancia G, Fagard R, Narkiewicz K, Redon J, Zanchetti A, Bohm M, et al. 2013 ESH/ ESC Guidelines for the management of arterial hypertension: The Task Force for the management of arterial hypertension of the European Society of Hypertension (ESH) and of the European Society of Cardiology (ESC). J Hypertens. 2013; 31(7): 1281-357. DOI: https://doi.org/10.1097/01.hjh.0000431740.32696.cc

15. O'Leary DH, Bots ML. Imaging of atherosclerosis: Carotid intima-media thickness. Eur Heart J. 2010; 31(14): 1682-9. DOI: https://doi.org/10.1093/eurheartj/ehq185

16. Ruijter HMD, Peters SAE, Anderson TJ, Britton AR, Dekker JM, Eijkemans MJ, et al. Common carotid intima-media thickness measurements in cardiovascular risk prediction: A meta-analysis. JAMA. 2012; 308(8): 796-803. DOI: https://doi.org/10.1001/ jama.2012.9630

17. Chen LY, Leening MJ, Norby FL, Roetker NS, Hofman A, Franco OH, et al. Carotid intima-media thickness and arterial stiffness and the risk of atrial fibrillation: The Atherosclerosis Risk in Communities (ARIC) study, Multi-Ethnic Study of Atherosclerosis (MESA), and the Rotterdam Study. J Am Heart Assoc. 2016; 5(5). DOI: https://doi.org/10.1161/JAHA.115.002907

18. Jin-Kee $\mathrm{P}$, Hyuntae $\mathrm{P}$, Kwi-Baek K. The relationship between distribution of body fat mass and carotid artery intima-media thickness in Korean older adults. J Phys Ther Sci. 2015; 27(10): 3141-6. DOI: https://doi.org/10.1589/jpts.10.3141

19. Kawamoto R, Ohtsuka N, Ninomiya D, Nakamura S. Association of obesity and visceral fat distribution with intima-media thickness of carotid arteries in middle-aged and older persons. Internal Medicine. 2008; 47(3): 143-9. DOI: https://doi.org/10.2169/ internalmedicine.47.0478

20. Oike $M$, Yokokawa H, Fukuda H, Haniu T, Oka F, Hisaoka T, et al. Association between abdominal fat distribution and atherosclerotic changes in the carotid artery. Obes Res Clin Pract. 2014; 8(5): e448-58. DOI: https://doi.org/10.1016/j.orcp.2013.09.002

21. Lin A, Lacy ME, Eaton C, Correa A, Wu W-C. Inflammatory obesity phenotypes, gender effects and subclinical atherosclerosis in African Americans: The Jackson Heart Study. Arterioscler Thromb Vasc Biol. 2016; 36(12): 2431-8. DOI: https://doi.org/10.1161/ ATVBAHA. 116.307728

22. Ramsay M, Sankoh $O$, as members of the AWI-Gen study and the H3Africa Consortium. African partnerships through the H3Africa Consortium bring a genomic dimension to longitudinal population studies on the continent. Int J Epidemiol. 2016; 45(2): 305-8. DOI: https://doi.org/10.1093/ije/ dyv187

23. Ramsay M, Crowther NJ, Tambo E, Agongo G, Baloyi V, Dikotope S, et al. H3Africa AWIGen collaborative centre: a resource to study the interplay between genomic and environmental risk factors for cardiometabolic diseases in four sub-Saharan African countries. Glob Health Epidemiol Genom. 2016; 1(e20): 1-13. DOI: https://doi.org/10.1017/ gheg.2016.17 
24. Ali SA, Soo C, Agongo G, Alberts M, Amenga-Etego L, Boua RP, et al. Genomic and environmental risk factors for cardiometabolic diseases in Africa: methods used for Phase 1 of the AWI-Gen population cross-sectional study. Glob Health Action. 2018; 11(sup2): 1507133. DOI: https://doi.org/10.1080 /16549716.2018.1507133

25. Richter L, Norris S, Pettifor J, Yach D, Cameron N. Cohort profile: Mandela's children: The 1990 Birth to twenty study in South Africa. Int J Epidemiol. 2007; 36(3): 504-11. DOI: https://doi.org/10.1093/ije/dym016

26. Gomez-Olive FX, Montana L, Wagner RG, Kabudula CW, Rohr JK, Kahn K, et al. Cohort Profile: Health and ageing in Africa: A longitudinal study of an INDEPTH community in South Africa (HAALSI). Int J Epidemiol. 2018 (1464-3685 (Electronic)). DOI: https:// doi.org/10.1093/ije/dyx247

27. Alberts M, Dikotope SA, Choma SR, Masemola ML, Modjadji SEP, Mashinya F, et al. Health \& demographic surveillance system profile: The Dikgale health and demographic surveillance system. Int J Epidemiol. 2015; 44(5): 1565-71. DOI: https://doi.org/10.1093/ ije/dyv157

28. Beguy D, Elung'ata P, Mberu B, Oduor C, Wamukoya M, Nganyi B, et al. Health \& demographic surveillance system profile: The Nairobi Urban Health and Demographic Surveillance System (NUHDSS). Int J Epidemiol. 2015; 44(2): 462-71. DOI: https://doi. org/10.1093/ije/dyu251

29. Derra K, Rouamba E, Kazienga A, Ouedraogo S, Tahita MC, Sorgho H, et al. Profile: Nanoro health and demographic surveillance system. Int J Epidemiol. 2012; 41(5): 1293-301. DOI: https://doi. org/10.1093/ije/dys159

30. Oduro AR, Wak G, Azongo D, Debpuur C, Wontuo P, Kondayire F, et al. Profile of the Navrongo health and demographic surveillance system. Int J Epidemiol. 2012; 41(4): 968-76. DOI: https://doi. org/10.1093/ije/dys111

31. Dogan S, Plantinga Y, Crouse JR, Evans GW, Raichlen JS, O'Leary DH, et al. Algorithms to measure carotid intima-media thickness in trials: A comparison of reproducibility, rate of progression and treatment effect. J Hypertens. 2011; 29(11): 2181-93. DOI: https:// doi.org/10.1097/ HJH.0b013e32834b0eba

32. Touboul PJ, Hennerici MG, Meairs S, Adams H, Amarenco P, Bornstein N, et al. Mannheim carotid intima-media thickness and plaque consensus (2004-2006-2011). An update on behalf of the advisory board of the 3rd, 4th and 5th watching the risk symposia, at the 13th, 15th and 20th European Stroke Conferences, Mannheim, Germany, 2004, Brussels, Belgium, 2006, and Hamburg, Germany, 2011. Cerebrovasc Dis. 2012; 34(4): 290-6. DOI: https://doi.org/10.1159/000343145

33. Nambi V, Chambless L, He M, Folsom AR, Mosley T, Boerwinkle E, et al. Common carotid artery intima-media thickness is as good as carotid intima-media thickness of all carotid artery segments in improving prediction of coronary heart disease risk in the Atherosclerosis Risk in Communities (ARIC) study. Eur Heart J. 2012; 33(2): 183-90. DOI: https://doi.org/10.1093/eurheartj/ehr192

34. Stolk RP, Meijer R, Mali WP, Grobbee DE, van der Graaf Y. Ultrasound measurements of intra-abdominal fat estimate the metabolic syndrome better than do measurements of waist circumference. Am J Clin Nutr. 2003; 77(4): 857-60. DOI: https://doi.org/10.1093/ ajcn/77.4.857 
35. De Lucia Rolfe E, Norris SA, Sleigh A, Brage S, Dunger DB, Stolk RP, et al. Validation of ultrasound estimates of visceral fat in black South African adolescents. Obesity. 2011; 19(9): 1892-7. DOI: https:// doi.org/10.1038/oby.2011.213

36. Riley RD, Lambert PC, Abo-Zaid G. Meta-analysis of individual participant data: Rationale, conduct, and reporting. BMJ. 2010; 340: c221. DOI: https://doi.org/10.1136/ bmj.c221

37. Gast KB, den Heijer M, Smit JW, Widya RL, Lamb HJ, de Roos A, et al. Individual contributions of visceral fat and total body fat to subclinical atherosclerosis: The NEO study. Atherosclerosis. 2015; 241(2): 547-54. DOI: https://doi.org/10.1016/j. atherosclerosis.2015.05.026

38. Wildman RP, Janssen I, Khan UI, Thurston R, Barinas-Mitchell E, El Khoudary SR, et al. Subcutaneous adipose tissue in relation to subclinical atherosclerosis and cardiometabolic risk factors in midlife women. Am J Clin Nutr. 2011; 93(4): 719-26. DOI: https://doi.org/10.3945/ajcn.110.007153

39. Fantuzzi G, Mazzone T. Adipose tissue and atherosclerosis: exploring the connection. Arterioscler Thromb Vasc Biol. 2007; 27(5): 996-1003. DOI: https://doi.org/10.1161/ ATVBAHA.106.131755

40. Mudie K, Lawlor DA, Pearce N, Crampin A, Tomlinson L, Tafatatha T, et al. How does the association of general and central adiposity with glycaemia and blood pressure differ by gender and area of residence in a Malawian population: A cross-sectional study. Int J Epidemiol. 2018; 47(3): 887-98. DOI: https://doi.org/10.1093/ije/dyy047

41. Sinabulya I, Kayima J, Longenecker C, Luwedde M, Semitala F, Kambugu A, et al. Subclinical atherosclerosis among HIV-infected adults attending HIV/AIDS care at two large ambulatory HIV clinics in Uganda. PLoS One. 2014; 9(2): e89537. DOI: https:// doi.org/10.1371/journal.pone.0089537

42. van der Merwe MT, Crowther NJ, Schlaphoff GP, Gray IP, Joffe BI, Lonnroth PN. Evidence for insulin resistance in black women from South Africa. Int J Obes Relat Metab Disord. 2000; 24(10): 1340-6. DOI: https://doi.org/10.1038/sj.ijo.0801416

43. Waisberg R, Paiker JE, Crowther NJ. Adipokine serum concentrations, anthropometric measurements and socio-economic status in two ethnic groups with different prevalence levels for cardiovascular diseases and type 2 diabetes. Horm Metab Res. 2011; 43(9): 660-6. DOI: https://doi. org/10.1055/s-0031-1283139

44. Lovren $F$, Teoh $H$, Verma S. Obesity and atherosclerosis: Mechanistic insights. Can $J$ Cardiol. 2015; 3 1(2): 177-83. DOI: https://doi.org/10.1016/j.cjca.2014.11.031

45. Narumi H, Yoshida K, Hashimoto N, Umehara I, Funabashi N, Yoshida S, et al. Increased subcutaneous fat accumulation has a protective role against subclinical atherosclerosis in asymptomatic subjects undergoing general health screening. International Journal of Cardiology. 2009; 135(2): 150-5. DOI: https://doi.org/10.1016/j. ijcard.2008.03.044

46. Bouchi R, Takeuchi T, Akihisa M, Ohara N, Nakano Y, Nishitani R, et al. High visceral fat with low subcutaneous fat accumulation as a determinant of atherosclerosis in patients with type 2 diabetes. Cardiovasc Diabetol. 2015; 14: 136. DOI: https://doi. org/10.1186/s12933-015-0302-4 
47. Chen $P$, Hou X, Hu G, Wei L, Jiao L, Wang H, et al. Abdominal subcutaneous adipose tissue: a favorable adipose depot for diabetes? Cardiovasc Diabetol. 2018; 17(1): 93. DOI: https://doi.org/10.1186/ sl2933-018-0734-8

48. Porter SA, Massaro JM, Hoffmann U, Vasan RS, O'Donnell CJ, Fox CS. Abdominal subcutaneous adipose tissue: a protective fat depot? Diabetes Care. 2009; 32(6): 1068-75. DOI: https://doi. org/10.2337/dc08-2280

49. Frayn KN. Adipose tissue as a buffer for daily lipid flux. Diabetologia. 2002; 45(9): 1201-10. DOI: https://doi.org/10.1007/s00125-002-0873-y

50. Kohli S, Sniderman AD, Tchernof A, Lear SA. Ethnic-specific differences in abdominal subcutaneous adipose tissue compartments. Obesity. 2010; 18(11): 2177-83. DOI: https://doi. org/10.1038/oby.2010.94

51. Marinou K, Hodson L, Vasan SK, Fielding BA, Banerjee R, Brismar K, et al. Structural and functional properties of deep abdominal subcutaneous adipose tissue explain its association with insulin resistance and cardiovascular risk in men. Diabetes Care. 2014; 37(3): 821-9. DOI: https://doi. org/10.2337/dcl3-1353

52. Wu TW, Hung CL, Liu CC, WU YJ, Wang LY, Yeh HI. Associations of cardiovascular risk factors with carotid intima-media thickness in middle-age adults and elders. J Atheroscler Thromb. 2017; 24(7): 677-86. DOI: https://doi.org/10.5551/jat.37895

53. Chang $E$, Varghese $M$, Singer K. Gender and sex differences in adipose tissue. Curr Diab Rep. 2018; 18(9): 69. DOI: https://doi.org/10.1007/s11892-018-1031-3 


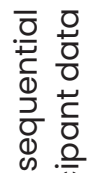

号

क

क

ำ 음

ठำ

드.

क ल

ธั ह

이은

흐

$\stackrel{1}{\subseteq}$

可

을 일

ह

O

过 \&

ญ

क क क

$\stackrel{\oplus}{\Perp} \stackrel{\oplus}{\infty}$

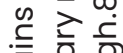

i के के

วิ

은 흥ํㅇ

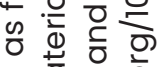

등ำ वे

ริำ के

¿훙하

过的是

ठํㅇ웡

응 응하음

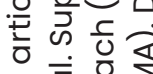

을 흥

돈 은 응

흔 흔 응응

幽 $\overline{0}$

음 它苍紊

들 을 응

등 응 흥

은 응 윽응 눙

흥 ठ

운

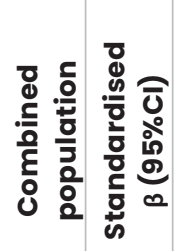

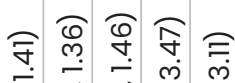

ने ले के के

○ 0 巳 $\stackrel{\infty}{=}$

б

$\begin{array}{lllll}0 & \infty & 0 & 0 & 0 \\ & 0 & 0 & \text { N } & \text { N }\end{array}$

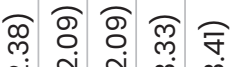

i v $v$ ले

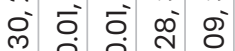

e 11 i 00

๗ே
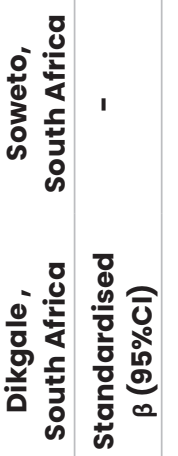

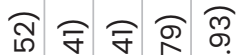
ल ल ल ले

官

$\stackrel{\oplus}{=} \doteq 0$

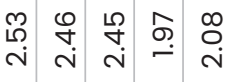

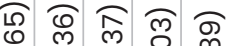

i $\sim$ v

ळ กั

e $\dot{0}$ e 0

ㄷ. 뭄

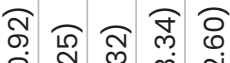

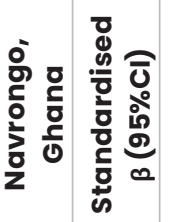

० N m

กั ฯ

गे

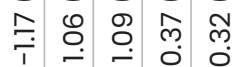

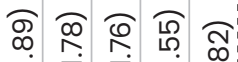

$\begin{array}{lllll}\infty & \infty & 0 & \infty & \cdots \\ \infty & \infty & \infty & 0 & \cdots\end{array}$

- 0 e 0

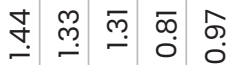

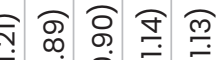

品

¿

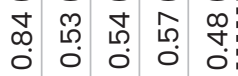

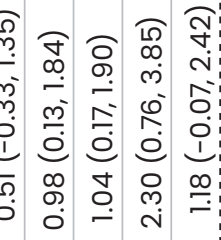

กิ ণิ ণิ ช

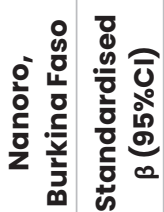

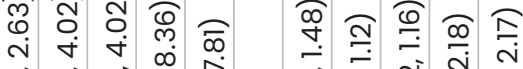

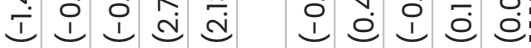

ส

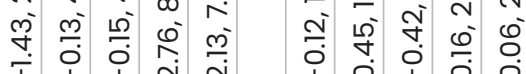

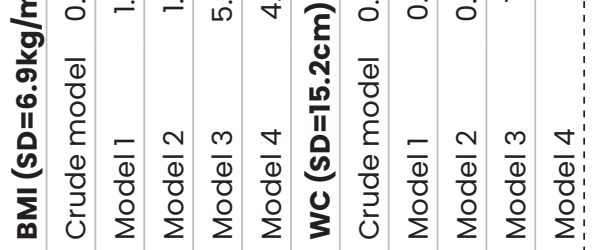




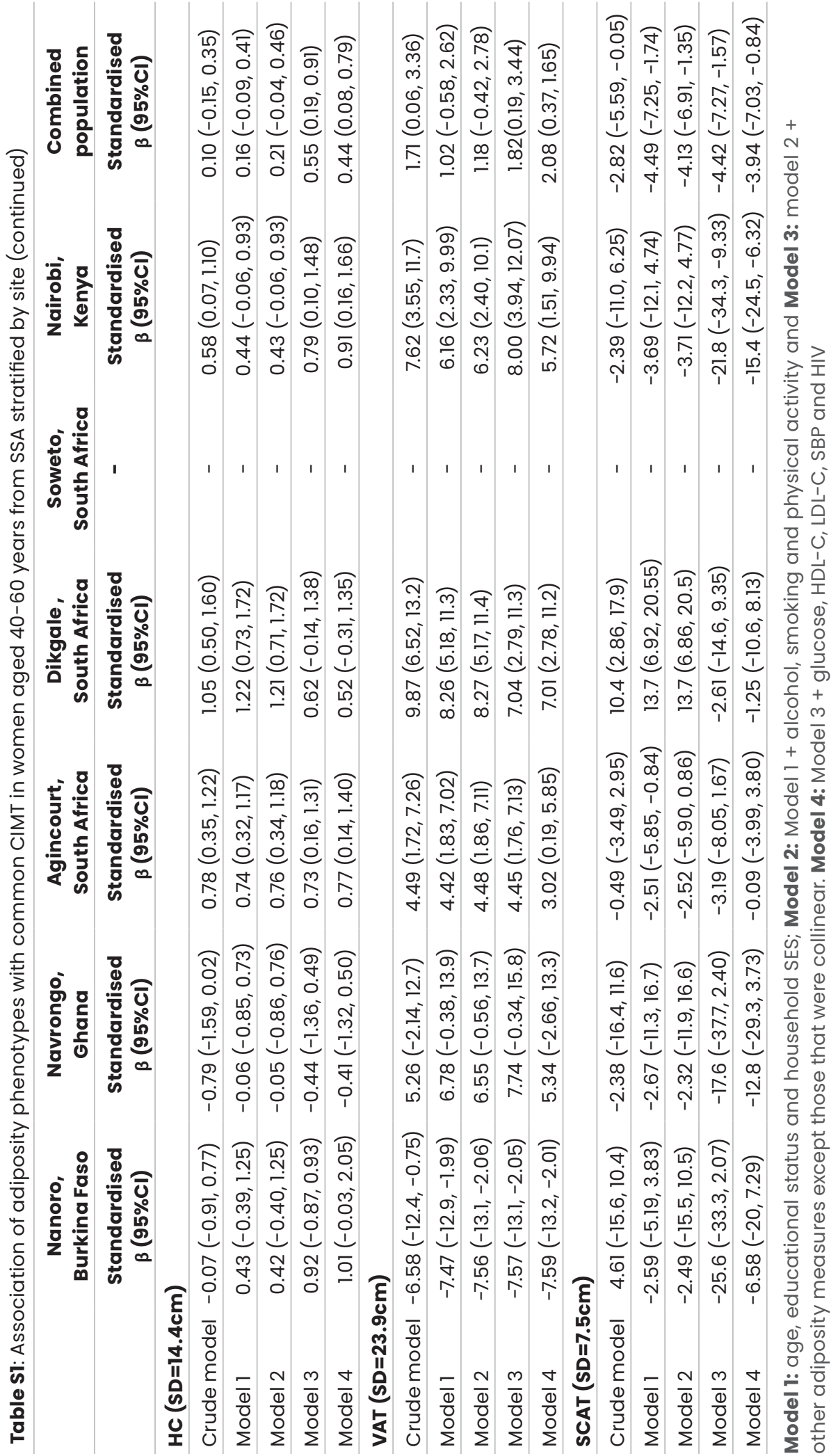




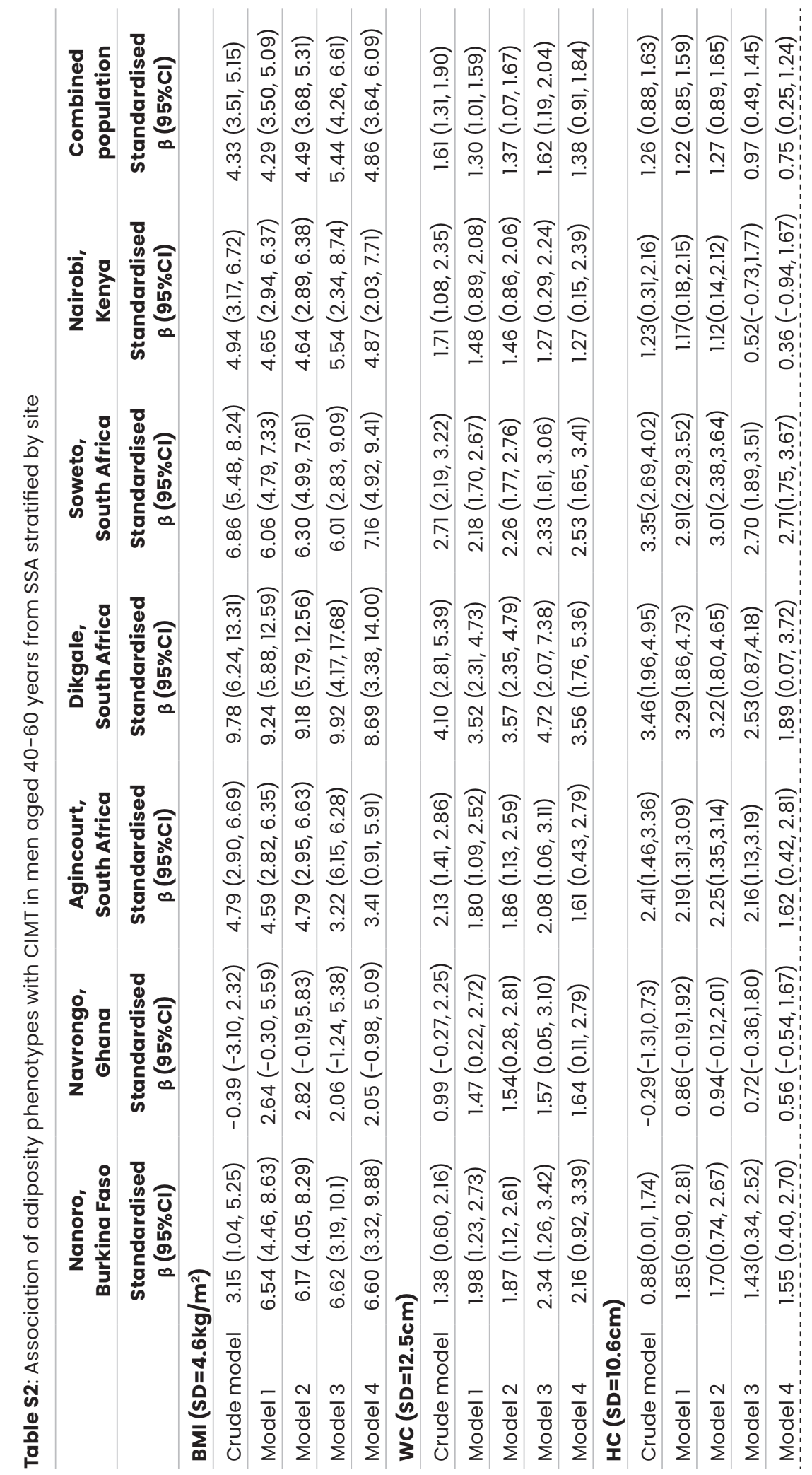




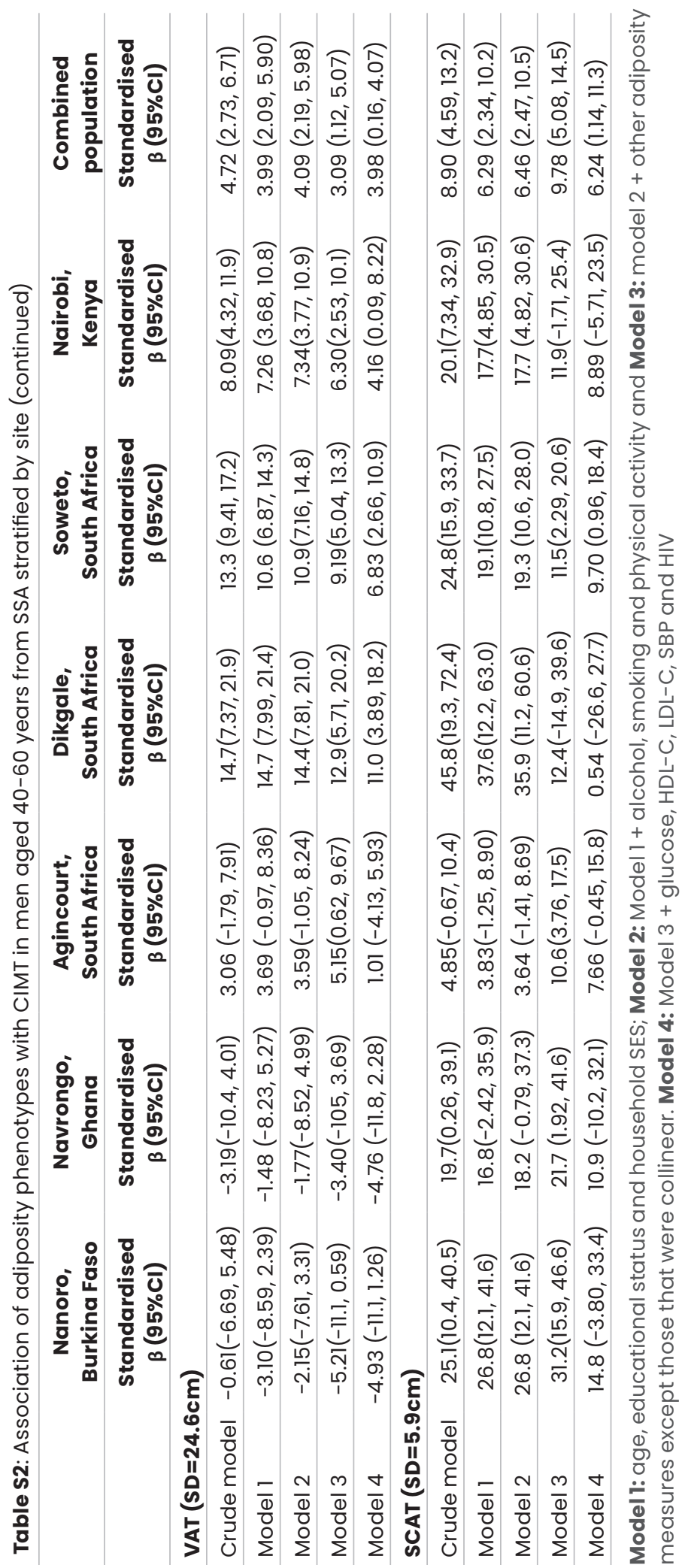




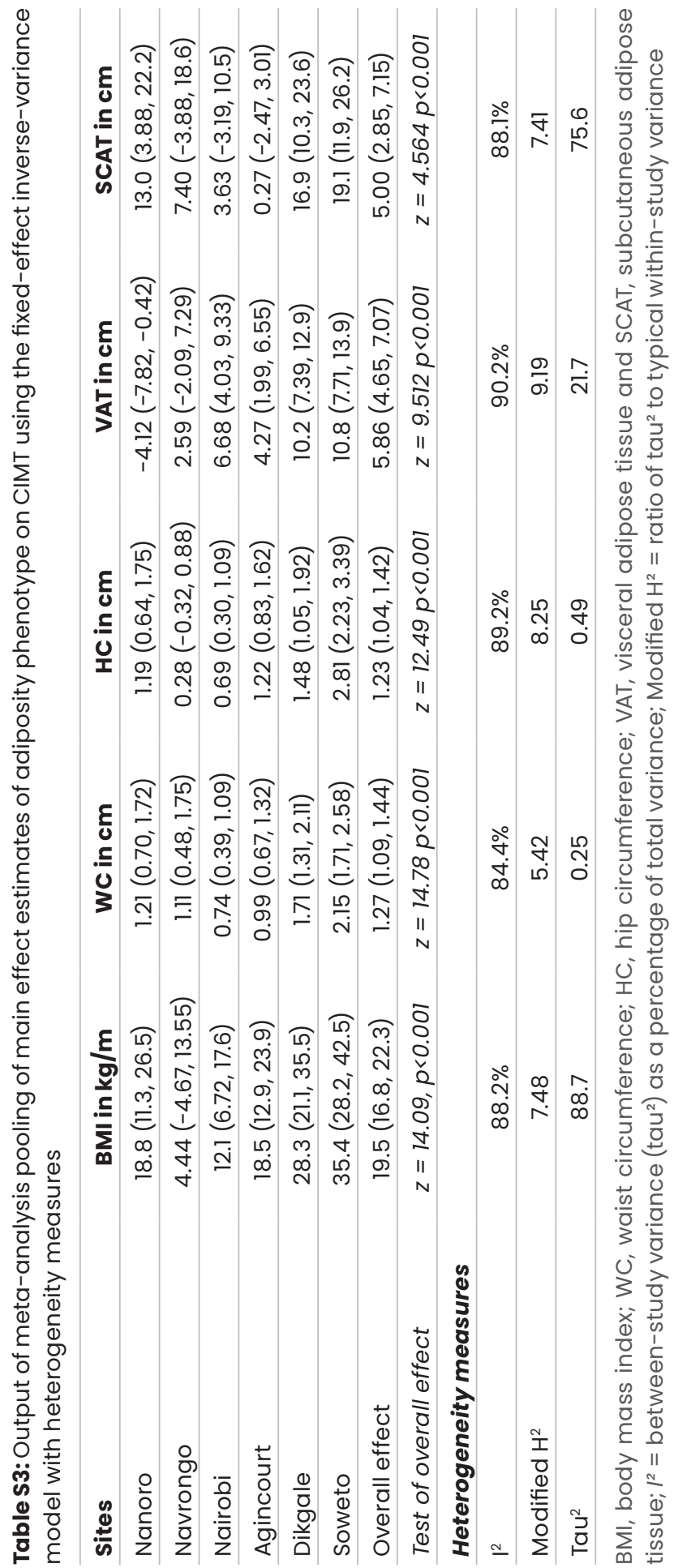



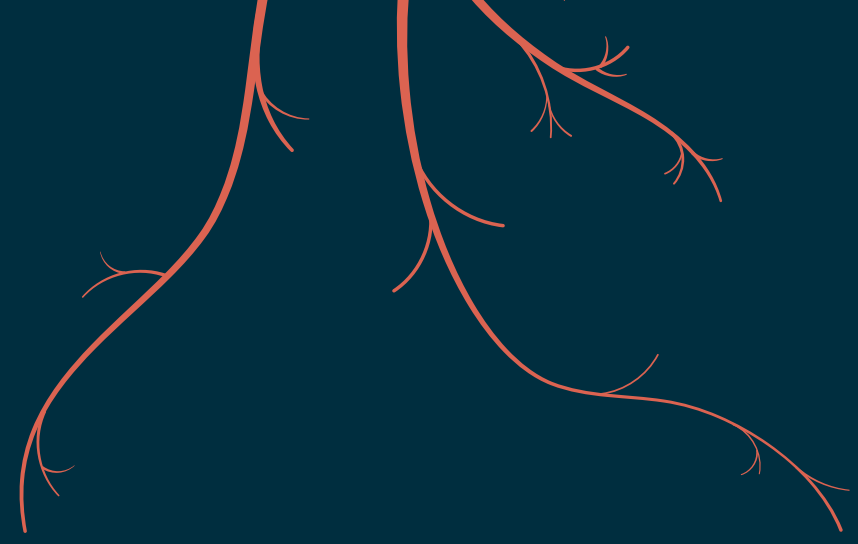

\section{Chapter 4}

\section{Race-ethnic differences in the} association between classical cardiovascular risk factors and common carotid intima-media thickness: an individual participant data meta-analysis

Nonterah EA, Crowther NJ, Klipstein-Grobusch K, Oduro AR, Kavousi M, Agongo G, Anderson TJ, Asiki G, Boua PR, Choma SSR, Couper DJ, Engström G, de Graaf J, Kauhanen J, Lonn EM, Mathiessen EB, Micklesfield LK, Okazaki S, Polak JF, Rundek T, Salonen JT, Tollman SM, Tuomainen T-P, Grobbee DE, Ramsay M, and Bots ML, for the H3Africa AWI-Gen and USE-IMT collaborative study group. 


\section{Abstract}

Background: The major risk factors for atherosclerotic cardiovascular disease (CVD) differ by race-ethnicity, but have largely been defined using populations of European ancestry. Despite the rising prevalence of CVD in Africa there is little related data from African populations. Therefore, we compared the association of established cardiovascular risk factors with carotid-intima media thickness (CIMT), a subclinical marker of atherosclerosis, between African, African American, Asian, European and Hispanic populations.

Methods: Cross sectional analyses of 34,025 men and women drawn from 15 cohorts in Africa, Asia, Europe, and North America was undertaken. Classical cardiovascular risk factors were assessed and CIMT measured using B-mode ultrasound. Ethnic differences in the association of established cardiovascular risk factors with CIMT were determined using a two-stage individual participant data meta-analysis (IPD-MA) with beta coefficients expressed as a percentage using the White population as the reference group.

Results: CIMT adjusted for risk factors was the greatest among African Americans followed by Asian, European, and Hispanic populations with Africans having the lowest mean CIMT. In all race-ethnic groups, men had higher CIMT levels compared to women. Age, sex, body mass index (BMI) and systolic blood pressure (SBP) had a significant positive association with CIMT in all ethnicities at varying magnitudes. When compared to European populations, the association of age, sex and SBP with CIMT was weaker in all ethnicities. Smoking (beta coefficient [95\% Cl]: $0.39[0.09,0.70])$, BMI $(0.05[0.01,0.08])$ and glucose $(0.13[0.06,0.19])$ had the strongest positive association with CIMT in the Asian population when compared to all other ethnic groups. High density lipoprotein-cholesterol (HDL-C) had significant protective effects in African American $(-0.31[-0.42,-0.21])$ and African $(-0.26[-0.31,-0.19])$ populations only.

Conclusion: The strength of association between established cardiovascular risk factors and CIMT differed across the race-ethnic groups and may be due to lifestyle risk factors and genetics. These differences have implications for race-ethnic specific primary prevention strategies and also give insights into the differential contribution of risk factors to the aetiology of CVD. The greatest burden of subclinical atherosclerosis in African Americans warrants further investigations.

Key words: Race; ethnicity; carotid intima-media thickness; atherosclerosis; cardiovascular disease risk; individual participant data meta-analysis 


\section{Introduction}

Cardiovascular disease (CVD) is the primary cause of deaths globally $(1,2)$. Differences in the prevalence of the classical risk factors between ethnic groups have been reported to account for the differences in CVD burden (3). Previous studies examining ethnic differences in CVD events have been largely conducted outside of the African region mostly in migrant populations residing in high income countries (4-6).

The INTERHEART and PURE studies $(7,8)$ are the only studies that have included Africans residing in Africa. These studies demonstrated that the risk of myocardial infarction due to smoking, BMl, blood pressure and lipids did not differ by geographical location or ethnicity. The INTERHEART study included 578 Africans drawn from Mozambique, Nigeria, South Africa, Sudan, and Uganda while the PURE study included study participants from Zimbabwe and South Africa. The paucity of data from African countries is problematic as the prevalence of CVDs has been reported to be increasing across the continent $(2,8)$. In terms of primary prevention it is important to identify risk factors and the strength of their association with cardiovascular events or measures of atherosclerosis, and whether differences exist between ethnic groups for these associations.

Carotid intima-media thickness (CIMT) is a proxy for subclinical atherosclerosis, and is related to CVD risk (9). With regard to African-ancestry participants, most studies on CIMT have been conducted in African American populations $(4,6)$ with one in Africans residing in Africa (10). The recent USE-IMT analyses of ethnic differences in the association between cardiovascular risk factors and CIMT used African Americans as a proxy for endemic African populations (6).

The current study uses individual participant data meta-analyses (IPD-MA) utilizing data from the USE-IMT project (II) combined with the large pan-African Africa-Wits-INDEPTH Genomic (AWI-Gen) study (12), a Human Hereditary and Health in Africa (H3Africa) Collaborative Center, to investigate the association of classical cardiovascular risk factors with CIMT with the inclusion of a large panAfrican cohort. In this study we determined the differences in the association of classical cardiovascular risk factors with CIMT among Africans residing in Africa and African American, Asian, Hispanic and European populations. 


\section{Methods}

\section{Study population}

Africans participants were recruited for H3Africa AWI-Gen study, a populationbased cross-sectional study investigating genomic and environmental risks factors for cardiometabolic diseases across six African sites including in Burkina Faso and Ghana (West Africa); Kenya (East Africa) and South Africa (12). The six cohorts were drawn from five Health and Socio-Demographic Surveillance Sites (HDSS) of the International Network for Demographic Evaluation of Populations and their Health (INDEPTH) and adults of the same age from Soweto, South Africa. We included all 6 cohorts from the AWI-Gen study composed of adults aged 40-60 years old. The USE-IMT project is an individual participant data meta-analysis (IPD-MA) as described in detail elsewhere (11). The USE-IMT comprised of adult men and women recruited for 18 cohorts from various countries in Europe, Asia and North America.

In order to adequately compare the USE-IMT cohorts with the AWI-Gen cohorts, we applied an age restriction. We excluded 9 cohorts from the 18 cohorts contributing to USE-IMT based on participant's age less than 40 years or greater than 60 years. Further exclusions were based on missing data for biomarkers: glucose, low density lipoprotein cholesterol (LDL-C) and high density lipoprotein cholesterol (HDL-C). The final dataset included in this IPD-MA consisted of 34,025 adults aged 40-60 years drawn from 15 cohorts in 11 countries.

The following cohorts contributed the recruited participants of European ancestry: FATE, Firefighters and Their Endothelium Study in Canada (13); ARIC, Atherosclerosis Risk in Communities study (14); MESA, Multi-Ethnic Study of Atherosclerosis (15) and NOMAS, Northern Manhattan (16) Studies all from the USA; KIHD, Kuopio Ischemic Heart Disease Risk Factor Study in Finland (17); Malmö, Malmö Diet and Cancer Study in Sweden (18); Tromsø Study in Norway (19) and the NBS, Nijmegen Biomedical Study in the Netherlands (20). African Americans and Hispanics were recruited from the ARIC, MESA and NOMAS studies in USA while participants from the Asian ethnic group were recruited from the OSACA2, Osaka Follow-up Study for Carotid Atherosclerosis study in Japan (21) and migrant Asians (Chinese) from the NBS, ARIC and MESA studies from the USA (supplementary Table SI). Analyses were limited to participants with data on carotid intima-media thickness (CIMT) and the following established cardiovascular risk factors: age, sex, smoking, alcohol, physical activity, body mass index, blood pressure, fasting glucose, LDL-C and HDL-C.

Race and ethnicity were self-identified by the participants of the various cohorts. Most cohorts adopted separate questions about ethnicity (Hispanic/ 
Latino ethnicity) and race (white/black) since these are distinct concepts (22). In addition, some studies collected information on country of origin among all races and language spoken at home to help classify multi-racial individuals who may only identify with a single group (23). We used the sociocultural context of race and ethnicity variables to assign sufficient attribution to explanatory biological factors (and based on the literature), and reported on the relationships among pre-specified race-ethnic groups according to recent recommendations for use of race and ethnicity in research (24). White raceethnic group refers to participant of European ancestry while black race-ethnic group are participants of African ancestry classified as Africans residing in Africa and African Americans referring to Africans in the diaspora.

\section{Data harmonization}

Several steps were taken to harmonize all extracted data for the current analyses. Data included were cross-sectional baseline data from the various cohorts thus offsetting differences in study designs. We obtained the crude individual level data (for each cohort) on all variables giving us the opportunity to standardize units of measurements of the variables. Weight was measured in kilogram in all cohorts while height was measured in $\mathrm{cm}$ in the USE-IMT and $\mathrm{mm}$ in the AWI-Gen study and was converted to meters. Subsequent body mass index (BMI) was calculated as weight divided by height in meters squared. We converted all serum biochemical marker data (glucose, LDL-C and HDL-C) into $\mathrm{mmol} / \mathrm{L}$. Blood pressure was measured as systolic and diastolic pressures in $\mathrm{mmHg}$. Alcohol intake in the USE-IMT was reported as the number of beverages consumed per week while in the AWI-Gen study it was reported as never consumed; current non-problematic consumer; current problematic consumer; former consumer (12). We thus recoded the merged variable into two mutually exclusive variables as 0 "never consumed" and 1 "ever consumed" (i.e. reporting consumption of at least one beverage per week or current non-problematic consumer; current problematic consumer or former consumer). Smoking was coded as 0 , never smoked and 1, current or former smoker. Physical activity was measured differently within the various study cohorts. Some studies used questionnaires to assess leisure activity and moderate-to-vigorous physical activity (MVPA) in some detail, whilst others asked whether subjects engaged in any form of physical activity, the type and duration per day or week. Those who reported not engaging in any form of physical activity or lower than 150mins of MVPA per week were coded as 0 (no form of physical activity) and those who reported $\geq 150$ mins of MVPA per week or engaging in activities that require physical exertion (e.g. walking, cycling) or some form of physical activity for at least 4 hours per week or several times per week were categorized as 1 (some form of physical activity). 


\section{Common carotid artery intima-media thickness}

Images of CIMT obtained varied across cohorts and from two to six. Some cohorts measured one angle and others multiple angles. The outcome variable, CIMT was thus computed as an average of all available angles (from the number of angles; from the far wall; and from one or both sides of the neck) of the common CIMT measurements from each included cohort. All common CIMT values were used in the analysis, including values larger than $1.5 \mathrm{~mm}$, which are suggestive of plaque. The same protocol was used for the six cohorts in the H3Africa AWI-Gen study limiting variability in measurements while different protocols were used by the various USE-IMT cohorts. As absolute differences in common CIMT levels between AWI-Gen and the USE-IMT cohorts could arise from differences in methodology employed in obtaining measurements, we harmonized the mean common CIMT by computing cohort-specific standard $z$-scores using the formula, $z=(x-\mu) / \sigma$, where $x$ is the crude participant CIMT, $\mu$ is the populations mean CIMT, and $\sigma$ is the population standard deviation.

\section{Statistical analyses}

Participants were stratified by race-ethnic background and by cohorts and their characteristics were summarized using counts and frequencies for categorical data and means with standard deviation ( $\pm S D$ ) for normally distributed continuous data. We used mixed effect multilevel (ML) linear regression analyses to compute and compare mean (with 95\% confidence intervals) CIMT between the different race-ethnicities while adjusting for the classical CVD risk factors. We further compared mean differences between African Americans, West, East and South Africans. We also conducted similar sub-group analyses for populations of Asian and European ancestry. Adjustments were done in order to determine whether any variations in ethnic differences were due to differential patterning of CVD risk factors by ethnicity. Due to significant sexethnicity interactions in the combined sample, we presented mean differences in CIMT for the combined sample and for women and men separately.

To determine the association between the established cardiovascular risk factors and common CIMT z-score, we conducted a two-staged IPD-MA. In the first stage, we conducted a mixed-effect multilevel regression with a randomeffects approach for each original cohort. We adjusted for all the established CVD risk factors separately for each ethnic group. For the AWI-Gen cohorts, we also adjusted for HIV as its prevalence was high in East and South African cohorts and it is established to contribute to the risk of CVD (25). In the second stage, a fixed-effect inverse-variance pooled IPD-MA approach was used to determine ethnic differences in CVD risk factors associated with common CIMT $z$-score. The meta-analysis pooling of the main effects on mean CIMT of each risk factor enabled us to obtain the associations of each risk factor with CIMT 
across the ethnic groups. The IPD was conducted for each established classical CVD risk factor and in each analysis adjustments were made for the other established classical CVD risk factors. We further conducted pooled analyses for men and women separately due to sex*ethnicity interactions. For Africans in the AWI-Gen cohorts we tested for the significance of an HIV*sex interaction. This is because the epidemiology of HIV in SSA differs between women and men. There was no sex*HIV interaction, but sex-ethnicity interaction terms were significant. Due to similarities in the effect sizes of the risk factors with raw mean CIMT and CIMT z-scores, we reported the associated differences in CIMT in $\mathrm{mm}$.

To further quantify the differences in the association of each risk factor with common CIMT according to ethnicity, we conducted a mixed-effect ML regression with a random-effects approach for each original cohort. We then extended this model by adding the interaction terms for ethnicity with each CVD risk factor. Beta coefficients (with 95\% confidence intervals) for each risk factor in each ethnic group were obtained with populations of European ancestry as the reference group in all analyses. From the obtained effect measure (beta value) for each race-ethnic group we converted the beta value to a percentage, again using the European race-ethnic group as a reference and for whom the beta coefficient was set at $100 \%$. This enabled us to observe the difference in the magnitude of the association of the risk factors with CIMT for each ethnic group compared to the European race-ethnic group. The established classical cardiovascular risk factors that were adjusted for in all the inferential analyses were: age, sex, smoking, alcohol intake, body mass index (BMI), SBP, glucose, LDL-C and HDL-C. We used LDL-C (more atherogenic lipoprotein) in preference to total cholesterol due the universal use of LDL-C in treatment guidelines for CVD prevention (26-28). We further analysed whether $\mathrm{LDL}-\mathrm{C} / \mathrm{HDL}-\mathrm{C}$ ratio was more important than LDL-C and HDL-C in predicting CIMT. We first included all three markers in the same model and checked for significant collinearity using the variance inflation factor. We then compared the variance (adjusted $\mathrm{R}^{2}$ ) of the model that included both HDL-C and LDL-C with that of the model that included the ratio only. A two-sided $p<0.05$ is considered statistically significant in all inferential analyses. All statistical analyses were conducted in STATA v14.2 (StataCorp LP, Texas USA).

\section{Ethical approval}

The AWI-Gen study received ethics approval from the Human Research Ethics Committee of the University of the Witwatersrand, Johannesburg, South Africa (initial approval number: M121029 and renewed approval number: M170880) and cohort specific ethical approvals were also obtained. The USE-IMT study received ethics approval from the institutional review committee of the University Medical Centre Utrecht, Utrecht, the Netherlands. The individual U.S. studies received 
ethical approval from their respective ethics committees or institutional review boards. All participants within each individual study provided informed consent prior to recruitment. Approval for use of the data included in these analyses was provided by the principal investigators of the H3Africa AWI-Gen and USE-IMT through a signed data transfer agreement.

\section{Data availability and access}

Data for these analyses are made up of baseline data from the USE-IMT initiative and the H3Africa AWI-Gen studies. AWI-Gen data can be accessed from the European Genome-phenome Archive (www.ega459archive.org/datasets/ EGAD00001006425). The phenotype dataset accession IDs is EGAD00010001996. The procedure to obtain access to the USE-IMT data goes through contacting the principal investigator (M.L.B.). The PI will then contact the PIs of the individual cohorts to ask for access through cohort specific existing procedures.

\section{Results}

\section{Basic characteristics of cohorts by race-ethnic groups}

The study consisted of 15 cohorts drawn from Africa, Asia, Europe and North America. Baseline characteristics of the included cohorts are presented in supplementary Table S1 while baseline characteristics of participants by ethnicity are presented in Table 1 . A total of 34,025 individuals with a mean \pm SD age of $52 \pm 5$ years and crude CIMT of $0.69 \pm 0.14 \mathrm{~mm}$ were studied. Hispanics were older ( $54 \pm 5$ years) than other ethnic groups with Africans having the lowest mean age ( $50 \pm 6$ years). An approximate equal number of women and men were included in this meta-analysis. More men were smokers and current alcohol consumers than women across all ethnic groups. Africans (30\%) and African Americans (29\%) had the highest smoking rates with Asians (15\%) having the lowest rates. African Americans and Hispanics had the highest mean BMI while Africans and Asians reported the lowest levels. African women had higher mean BMI than their male counterparts while the opposite was observed in all other race-ethnic groups. Africans generally had lower levels of fasting glucose, $\mathrm{HDL}-\mathrm{C}$ and LDL-C compared to all other ethnicities. African Americans had the highest glucose and HDL levels while Europeans had the highest LDL-C levels (Table 1). 


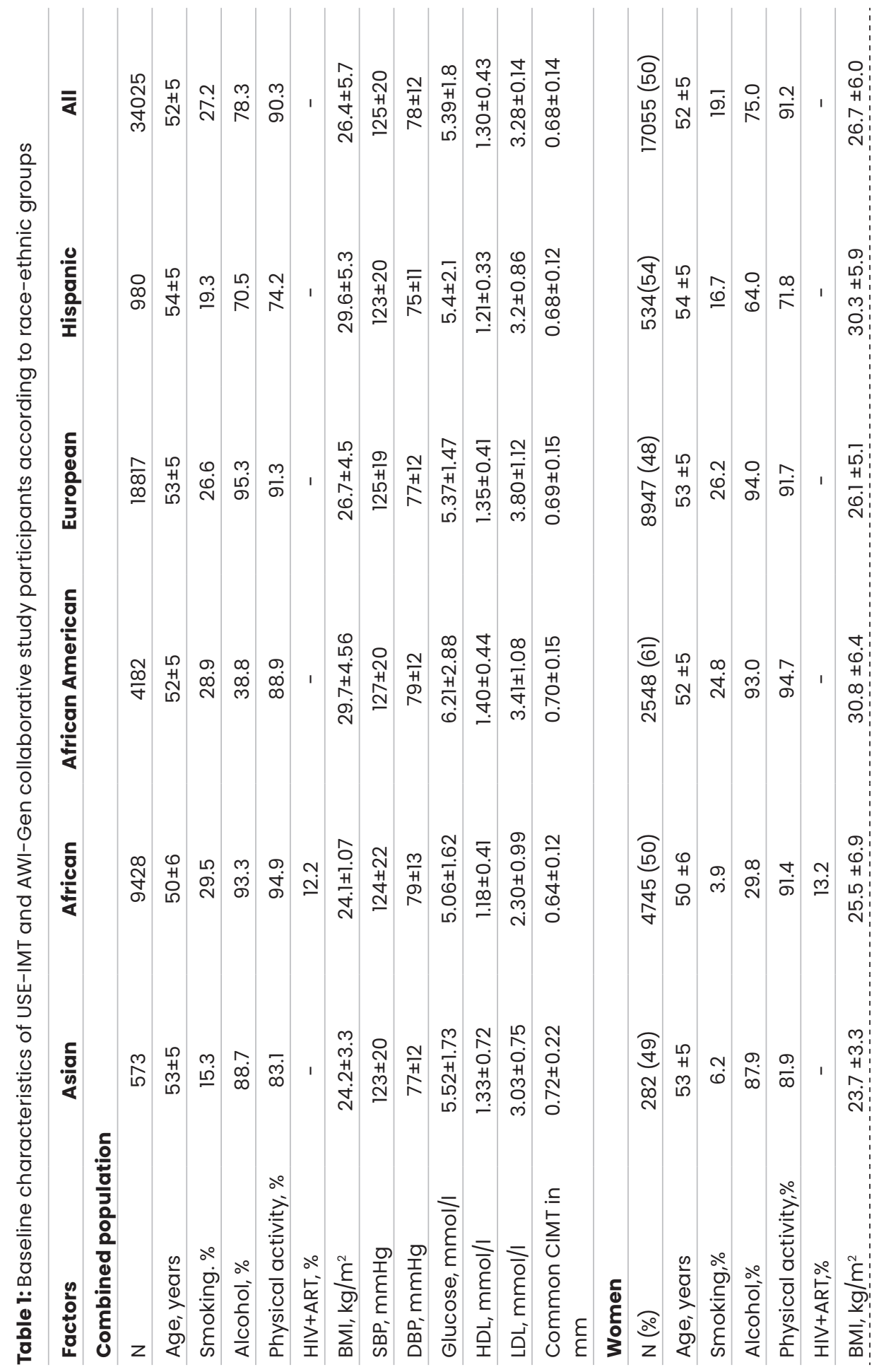




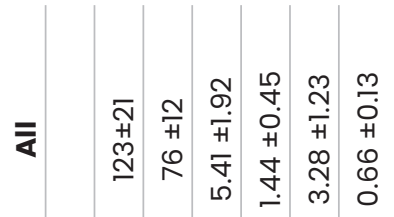

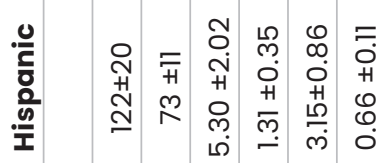

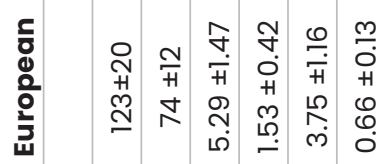

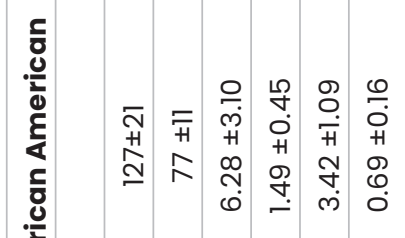

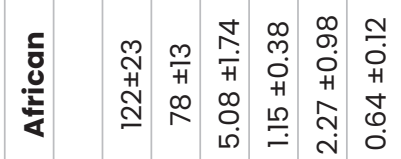

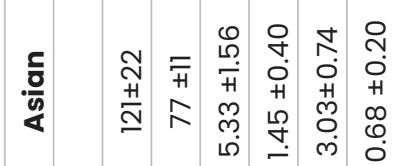

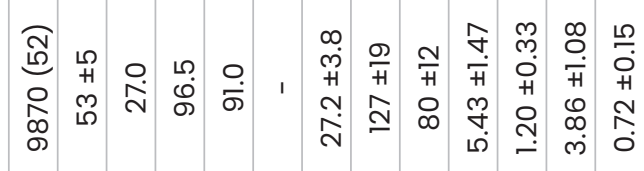

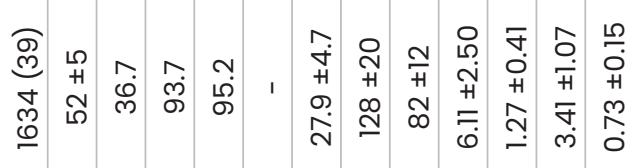

흠 뭉

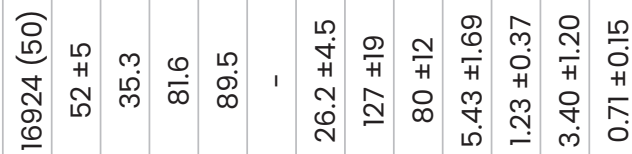

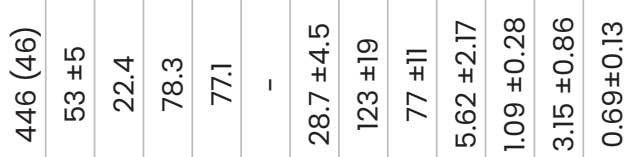

응

应

U一

希

is 1

ठ ำ

के है

응

$\sum_{\infty} \sum^{\varepsilon}$

竞引

흥

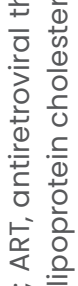

商

든 $\frac{1}{0}$

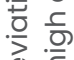

d

음 모

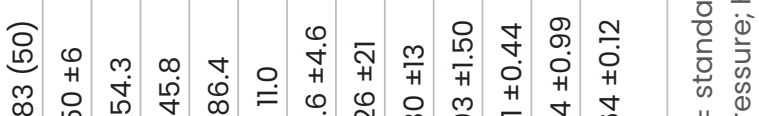

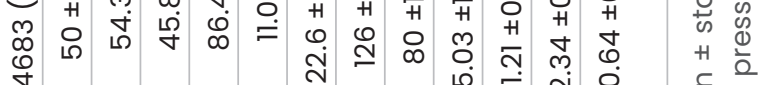

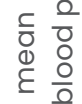

흐음

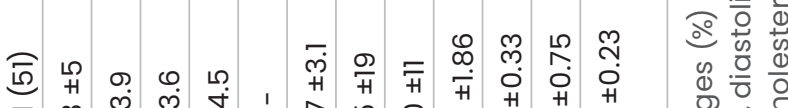

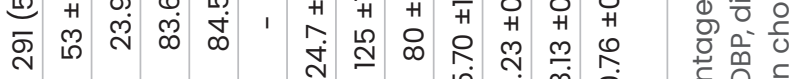

瓶

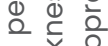

o

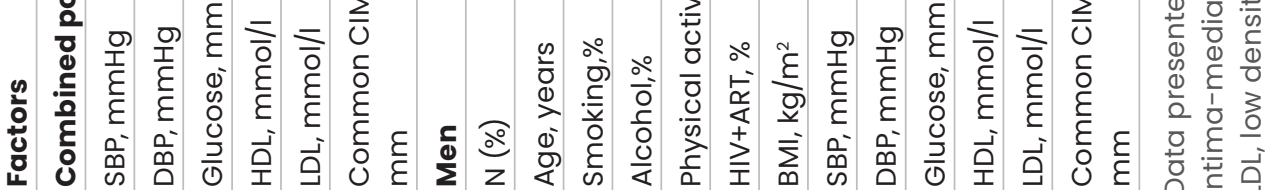




\section{Mean CIMT levels across race-ethnic groups}

The adjusted CIMT levels for each race-ethnic group as total, men and women separately are presented in Figure 1. In the combined populations, age, sex, smoking, alcohol, physical activity, glucose, HDL-C and LDL-C were adjusted sequentially (model 1, unadjusted; model 2, adjusted for age, sex and sex*ethnicity interaction; and model 3, model 2 plus the classical cardiovascular risk factors) to see the differential effect of behavioral and metabolic risk factors on mean common CIMT levels across the ethnicities. The results of these sequential models are presented in supplementary Table S2. The adjusted mean CIMT was highest among African Americans followed by Asian, White, and Hispanic populations with Africans having the lowest mean CIMT. There was a significant sex and race-ethnicity interaction, $p=0.001$ (supplementary Table s3) hence we conducted separate analyses for women and men.

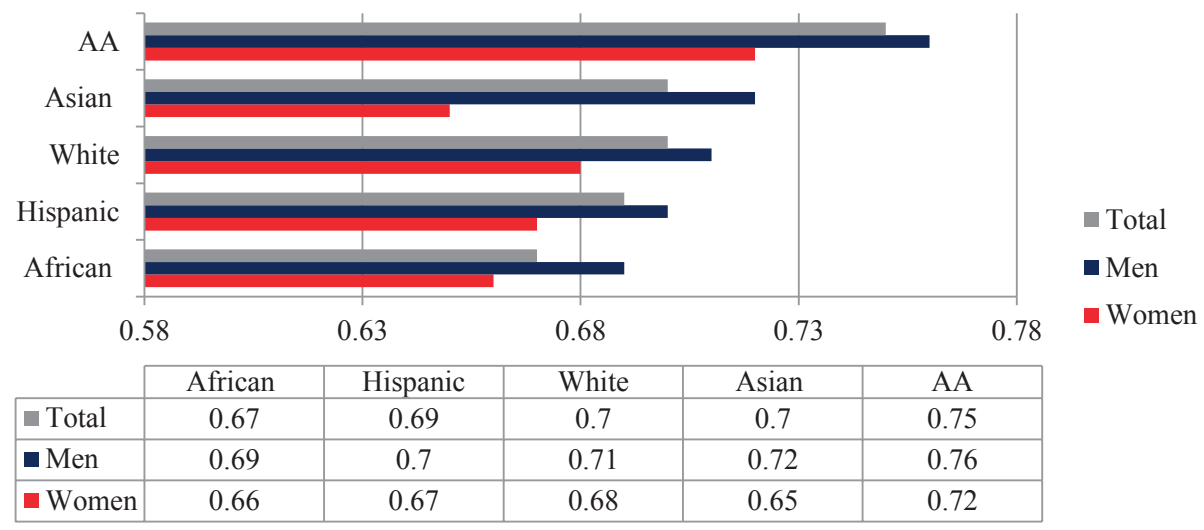

Figure 1: Adjusted means of common CIMT in mm (with $95 \% \mathrm{Cls}$ ) across the race-ethnic groups in the USE-IMT \& AWI-Gen collaborative study's combined sample. Values adjusted for age, sex (in the total sample), smoking, alcohol consumption, physical activity, systolic blood pressure, body mass index, high density lipoprotein cholesterol and low density lipoprotein cholesterol; AA, African American; white, European race-ethnic group

Men from all the ethnic groups had higher adjusted CIMT compared to women. African American women had the highest adjusted mean CIMT levels followed by African, European and Hispanic women with Asian women recording the lowest adjusted CIMT levels. A similar trend was observed in men except that Asian men had the second highest CIMT level. We further performed a subgroup analysis among African-ancestry participants by comparing mean CIMT levels between African Americans and East (Kenya), West (Ghana and Burkina Faso) and South Africans. In these analyses we observed that African Americans had the highest mean CIMT followed by West, South and East Africans (see Table 2). 
Table 2: Adjusted mean levels of carotid intima-media thickness (with $95 \% \mathrm{Cls}$ ) in participants with African ancestry including African Americans and East, West, and South Africans

\begin{tabular}{lccccc}
\hline & $\begin{array}{c}\text { African } \\
\text { American } \\
(\mathbf{n = 4 1 8 2 )}\end{array}$ & $\begin{array}{c}\text { West African } \\
(\mathbf{N}=\mathbf{4 0 9 0})\end{array}$ & $\begin{array}{c}\text { East African } \\
(\mathbf{N}=1940)\end{array}$ & $\begin{array}{c}\text { South African } \\
(\mathbf{N}=3398)\end{array}$ & $\begin{array}{c}\boldsymbol{P} \\
\text { interaction }\end{array}$ \\
\hline Combined sample & & & & \\
\hline Model 1 & $0.78(0.74,0.82)$ & $0.68(0.66,0.69)$ & $0.59(0.57,0.61)$ & $0.62(0.61,0.64)$ & - \\
\hline Model 2 & $0.78(0.74,0.81)$ & $0.68(0.66,0.69)$ & $0.60(0.58,0.62)$ & $0.62(0.61,0.63)$ & $<0.001$ \\
\hline Model 3 & $0.75(0.72,0.77)$ & $0.69(0.67,0.70)$ & $0.60(0.57,0.62)$ & $0.61(0.59,0.62)$ & $<.001$ \\
\hline Women & & & & & \\
\hline Model 1 & $0.76(0.72,0.81)$ & $0.67(0.65,0.69)$ & $0.60(0.56,0.63)$ & $0.62(0.60,0.65)$ & - \\
\hline Model 2 & $0.75(0.71,0.79)$ & $0.67(0.65,0.69)$ & $0.61(0.58,0.64)$ & $0.62(0.60,0.64)$ & - \\
\hline Model 3 & $0.72(0.68,0.75)$ & $0.68(0.66,0.70)$ & $0.60(0.58,0.63)$ & $0.61(0.59,0.62)$ & - \\
\hline Men & & & & & - \\
\hline Model 1 & $0.79(0.75,0.83)$ & $0.69(0.67,0.70)$ & $0.58(0.57,0.60)$ & $0.62(0.61,0.63)$ & - \\
\hline Model 2 & $0.78(0.75,0.82)$ & $0.68(0.67,0.69)$ & $0.59(0.57,0.61)$ & $0.62(0.61,0.63)$ & - \\
\hline Model 3 & $0.76(0.73,0.78)$ & $0.69(0.67,0.72)$ & $0.59(0.56,0.62)$ & $0.61(0.59,0.63)$ & - \\
\hline
\end{tabular}

Model 1 is crude unadjusted; model 2 is adjusted for age, sex and sex*ethnicity interaction (sex stratified analyses were adjusted for age only) and model 3 is model 2 with adjustment for smoking, alcohol, physical activity, SBP, BMI, glucose, HDL and LDL. $P_{\text {interaction' }} \mathrm{P}$-value for ethnicity*sex multiplicative interaction term

We also conducted sub-group analyses comparing adjusted mean CIMT levels of Chinese American with Japanese subjects (these two populations constitute the 'Asian' race-ethnic group in the present study) as well as European Americans with Europeans resident in Europe (these two populations constitute the 'European' race-ethnic group in the present study). In these analyses we observed that the CIMT level of Japanese subjects $(0.72(0.57,0.83) \mathrm{mm})$ was not significantly different to that of Chinese Americans $(0.73(0.67,0.79) \mathrm{mm})$. The CIMT for European subjects resident in Europe $(0.74(0.74,0.76) \mathrm{mm})$ was not significantly different from that of European Americans $(0.73(070,0.78) \mathrm{mm})$.

\section{Race-ethnic differences in association of CVD risk factors with common CIMT}

Results of the IPD-MAs to determine whether there were ethnic differences in the association of classical CVD risk factors with CIMT are presented as forest plots for the combined sample (Figure 2), women (Figure 3) and men (Figure 4). The data is also presented for the combined sample in Table 3 in which the beta coefficients for the associations with CIMT are expressed as percentages using the European race-ethnic group as a reference with a set value of $100 \%$. The measure of heterogeneity obtained from the IPD-MA is 
presented in supplementary Table S4. In the pooled IPD-MA for each risk factor with adjustment for other factors, we noticed that age, sex, BMI and SBP were significantly associated with CIMT in all ethnicities and at varying magnitudes (Table 3). The association between age and CIMT was significantly smaller in Africans (88\%), Hispanics (63\%) and Asians (50\%) but the same for African Americans when compared to European race-ethnic group. 


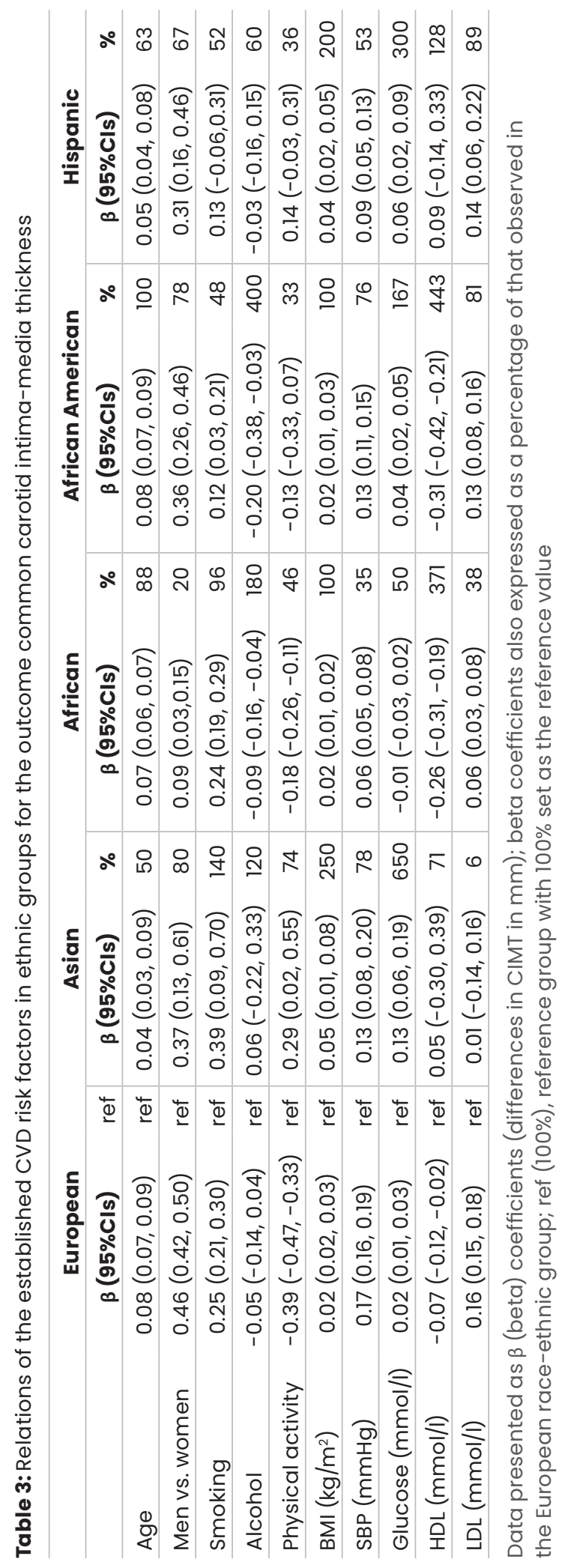


For all race-ethnic groups, SBP and men had lower beta coefficients compared to European race-ethnic group. Smoking was significantly associated with CIMT in all groups except for the Hispanic population, being strongest in the Asian group (140\%). Alcohol intake was inversely associated with common CIMT in all groups except Asians but was significant only in Africans (180\%) and African Americans (400\%). Physical activity was inversely associated with CIMT in Africans and European but positively associated with CIMT in Asians. Body mass index was significantly associated with CIMT in all population, being strongest in the Asian group (250\%).

The beta coefficients for the association between glucose and CIMT were highest in Asians (650\%), and were non-significant only in the African population. The association of HDL-C with CIMT was negative and significant in European, African and African-American populations whilst LDL-C was positively associated with common CIMT in all groups except Asians, being strongest in the European population. When analyzing whether the LDL-C/HDL-C ratio better predicted CIMT when compared to LDL and HDL, we noticed significant collinearity when LDL-C, HDL-C and LDL-C/HDL-C were included in the same model. We therefore split these models into two with $\mathrm{HDL}-\mathrm{C}$ and $\mathrm{LDL}-\mathrm{C}$ in one model and $\mathrm{LDL}-\mathrm{C} / \mathrm{HDL}-\mathrm{C}$ in the second. A comparison of the adjusted $\mathrm{R}^{2}$ indicated that $L D L-C / H D L-C$ ratio was no more predictive of CIMT than HDL-C and LDL-C (supplementary Table S5). In sex stratified analyses, similar associations of age, BMI and SBP with CIMT were observed for women and men except for SBP where Asian women (Figure 3) and Hispanic men displayed a marginal association (Figure 4). 


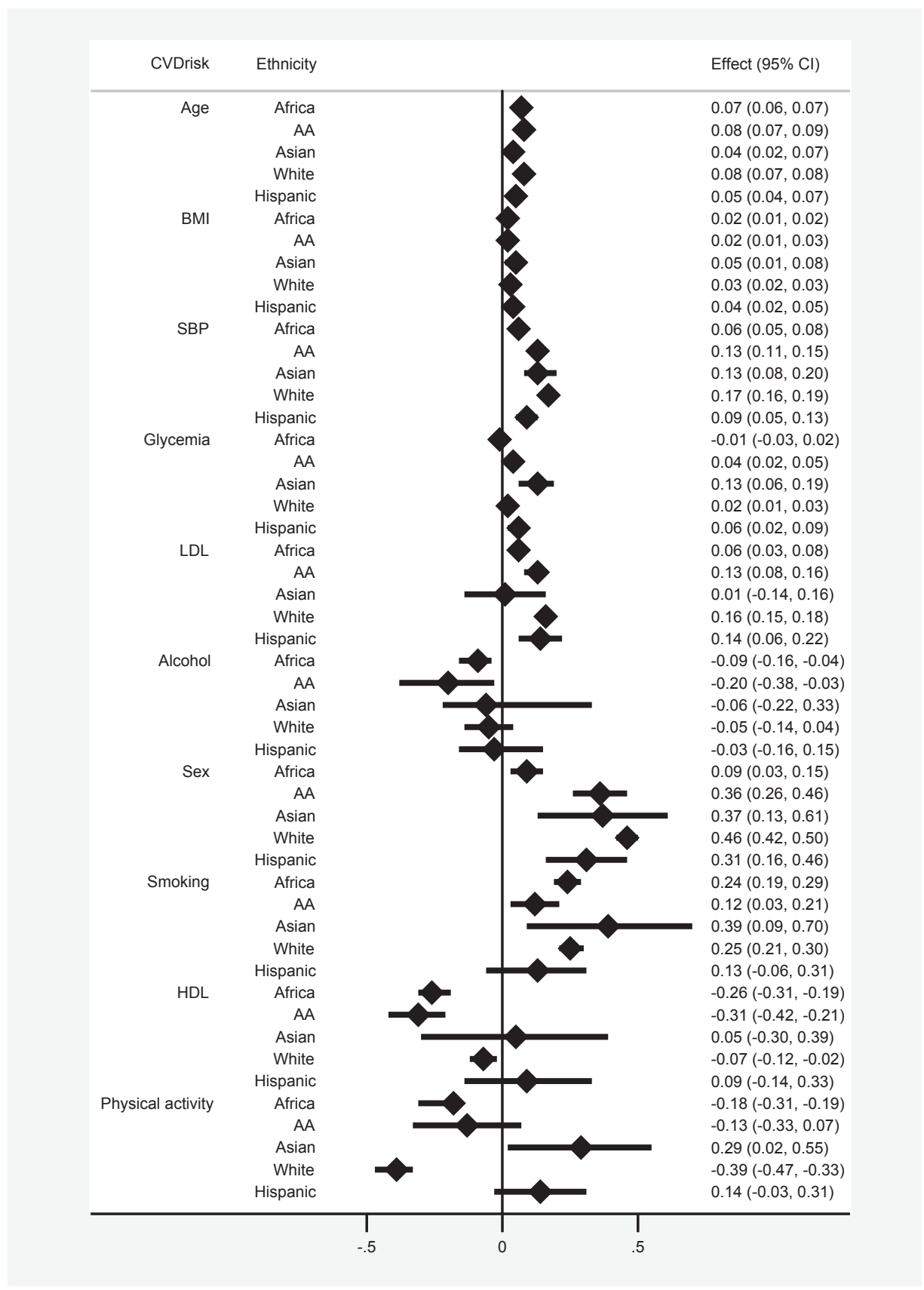

Figure 2: A forest plot of individual participant data meta-analysis of ethnic differences in the adjusted association between classical cardiovascular risk factors and common carotid intima-media thickness for the combined population; AA, African American; white, European race-ethnic group; sex, men vs. women; plots illustrate the beta coefficients with $95 \%$ confidence intervals. 


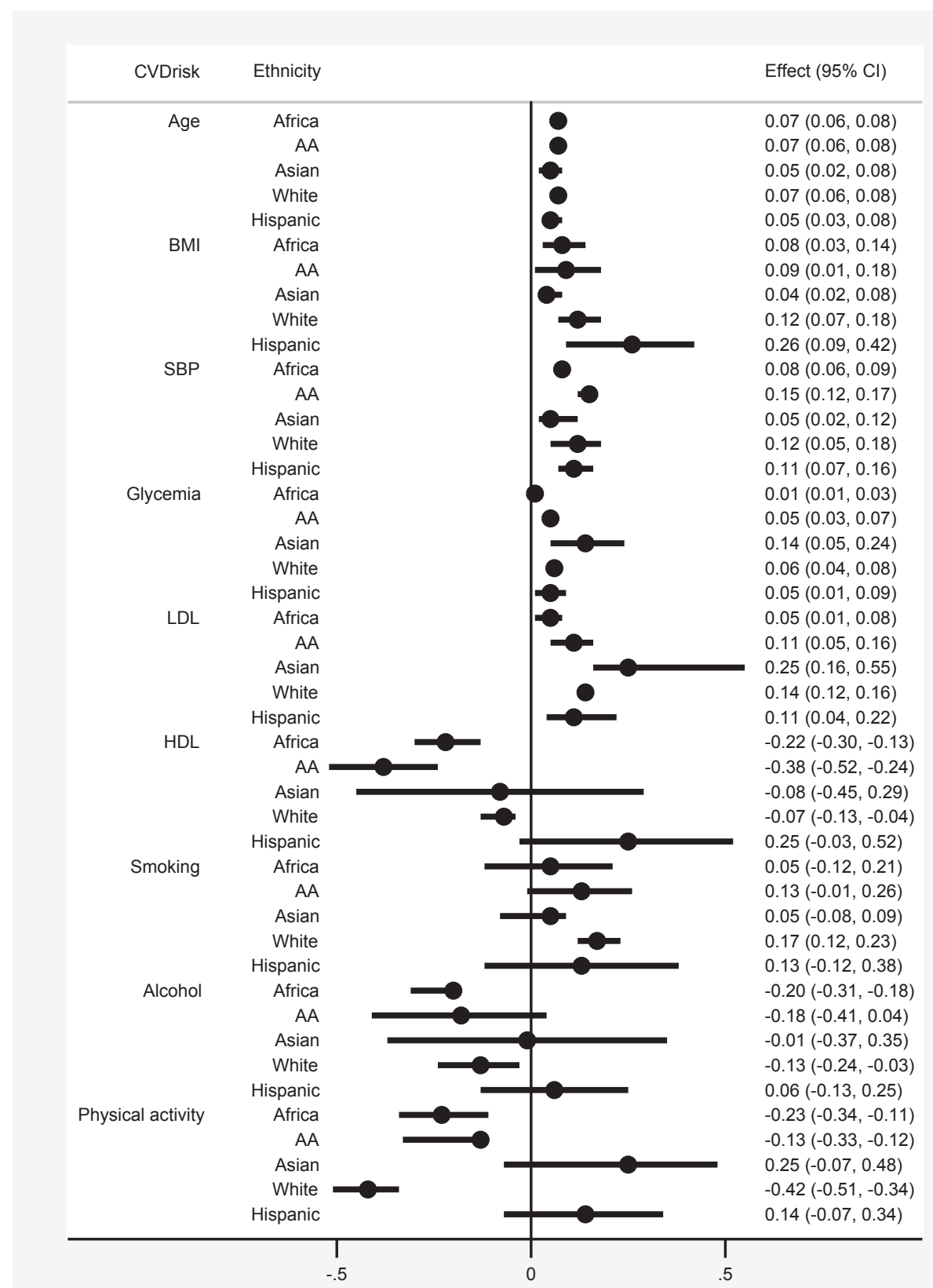

Figure 3: A forest plot of individual participant data meta-analysis of ethnic differences in the adjusted association between classical cardiovascular risk factors and common carotid intima-media thickness in women; AA, African American; white, European race-ethnic group; plots illustrate the beta coefficients with $95 \%$ confidence intervals. 


\begin{tabular}{|c|c|c|}
\hline CVDrisk & Ethnicity & Effect $(95 \% \mathrm{Cl})$ \\
\hline \multirow[t]{5}{*}{ Age } & Africa & $0.08(0.06,0.09)$ \\
\hline & $\mathrm{AA}$ & $0.09(0.08,0.11)$ \\
\hline & Asian & $0.04(0.07,0.08)$ \\
\hline & White & $0.09(0.08,0.11)$ \\
\hline & Hispanic & $0.05(0.02,0.08)$ \\
\hline \multirow[t]{5}{*}{ BMI } & Africa & $0.03(0.02,0.04)$ \\
\hline & $\mathrm{AA}$ & $0.04(0.03,0.06)$ \\
\hline & Asian & $0.04(0.02,0.11)$ \\
\hline & White & $0.04(0.02,0.04)$ \\
\hline & Hispanic & $0.06(0.04,0.09)$ \\
\hline \multirow[t]{5}{*}{ SBP } & Africa & $0.06(0.05,0.08)$ \\
\hline & AA & $0.11(0.08,0.14)$ \\
\hline & Asian & $0.30(0.17,0.42)$ \\
\hline & White & $0.19(0.17,0.20)$ \\
\hline & Hispanic & $0.05(0.01,0.12)$ \\
\hline \multirow[t]{5}{*}{ LDL } & Africa & $0.06(0.02,0.08)$ \\
\hline & AA & $0.15(0.08,0.21)$ \\
\hline & Asian & $0.06(-0.18,0.13)$ \\
\hline & White & $0.20(0.17,0.23)$ \\
\hline & Hispanic & $0.18(0.05,0.21)$ \\
\hline \multirow[t]{5}{*}{ Glycemia } & Africa & $-0.09(-0.32,0.14)$ \\
\hline & AA & $0.03(0.01,0.06)$ \\
\hline & Asian & $0.12(0.03,0.20)$ \\
\hline & White & $0.67(0.45,0.89)$ \\
\hline & Hispanic & $0.09(0.06,0.13)$ \\
\hline \multirow[t]{5}{*}{ HDL } & Africa & $-0.26(-0.33,-0.18)$ \\
\hline & AA & $-0.15(-0.32,0.03)$ \\
\hline & Asian & $0.15(-0.50,0.80)$ \\
\hline & White & $-0.12(-0.21,-0.03)$ \\
\hline & Hispanic & $-0.27(-0.71,0.17)$ \\
\hline \multirow[t]{5}{*}{ Smoking } & Africa & $0.08(0.01,0.14)$ \\
\hline & AA & $0.12(0.02,0.26)$ \\
\hline & Asian & $0.85(0.32,1.39)$ \\
\hline & White & $0.34(0.27,0.40)$ \\
\hline & Hispanic & $0.12(-0.15,0.39)$ \\
\hline \multirow[t]{5}{*}{ Alcohol } & Africa & $-0.14(-0.22,-0.06)$ \\
\hline & AA & $-0.24(-0.52,-0.03)$ \\
\hline & Asian & $0.09(-0.31,0.50)$ \\
\hline & White & $0.06(-0.09,0.21)$ \\
\hline & Hispanic & $-0.07(-0.34,0.19)$ \\
\hline \multirow[t]{6}{*}{ Physical activity } & Africa & $-0.12(-0.22,-0.02)$ \\
\hline & $A A$ & $-0.08(-0.39,0.26)$ \\
\hline & Asian & $0.29(-0.12,0.71)$ \\
\hline & White & $-0.38(-0.48,-0.28)$ \\
\hline & Hispanic & $0.11(-0.17,0.39)$ \\
\hline & & \\
\hline
\end{tabular}

Figure 4: A forest plot of individual participant data meta-analysis of ethnic differences in the adjusted association between classical cardiovascular risk factors and common carotid intima-media thickness for men; AA, African American; white, European race-ethnic group; plots illustrate the beta coefficients with $95 \%$ confidence intervals. 


\section{Discussion}

Our study involving a large cohort of adults from different race-ethnic groups shows that Africans, African Americans, Asians, Europeans and Hispanics differ markedly in the levels of CIMT and associated CVD risk factors. African Americans had significantly higher mean CIMT levels compared to all the other ethnic groups whilst among Africans, West Africans had higher levels of CIMT than East and South Africans. In the pooled individual participant data meta-analyses and adjusted multilevel analyses, age, sex, BMI, and SBP were significantly associated with CIMT in all race-ethnic groups but with the strength of the associations varying considerably. Compared to European populations, populations of African ancestry (African and African Americans) had higher strength of association between alcohol, HDL-C and CIMT while all other raceethnicities had a lower strength of association between LDL-C and CIMT.

It has been observed that race-ethnic differences in carotid artery thickness, diameter and stiffness are due to genetic factors as well as anatomical differences in the carotid artery (29) which may explain the higher CIMT in African American race-ethnic group in our study. African Americans also have disproportionately narrower internal and larger external carotid arteries compared to Asians, Europeans and Hispanics (29). This increases turbulent blood flow around the bifurcation resulting in high shear stress and exposure to risk factors enhances the thickening of the adjoining common carotid artery (29). African Americans are an admixed group with considerably variations in the relative proportions of African, European and other race-ethnic group ancestry between individuals. However, majority of African Americans are known to have largely originated from Yoruba, Bantu and Mandeka speaking individuals of West African origin, as well as from Central Africa, who were forcibly moved to North America as part of the slave trade $(30,31)$. The thicker CIMT in African Americans may therefore be due to their ancestry with West Africans who had thicker CIMT compared to the other African populations in the AWI-Gen study. In addition, the higher burden of established CVD risk factors among the African American compared to the African populations may be a possible contributor to the greater CIMT observed among the former group.

The independent association of age with increased atherosclerosis in all ethnic groups is consistent with published literature $(6,10)$. The common carotid artery is highly susceptible to the effects of aging with resultant thickening of the intima-media and increased vascular stiffness (32). Increasing life expectancy is therefore likely to result in higher CVD related morbidity and mortalities. The consistent finding of age effects on CIMT across all ethnic groups suggests that screening and management of risk factors should be targeted at older 
individuals in all populations. The positive association of common CIMT levels with smoking in all ethnicities has been previously reported $(7,8,10)$ as has the association of systolic blood pressure with high CIMT (6). High blood pressure is reported to cause detrimental effects on the vascular tree by inducing higher pressure overload causing arterial hypertrophy or hyperplasia (33).

The inverse association of alcohol intake with CIMT in Africans and African Americans could be attributed to genetic factors and the types of alcohol consumed. Allele frequencies of polymorphic loci in the gene that encodes one of the alcohol dehydrogenase isoforms (ADHIB) differ between populations of African and European ancestry and confer different levels of cardio-protection in both race-ethnicities. Thus, the alcohol-metabolizing ADH1B*3 functional allele, found almost exclusively in populations with African ancestry is associated with a higher conversion rate of ethanol to acetaldehyde $(34,35)$. This should not be regarded as a public health advocacy for the consumption of alcohol as a means of reducing lipid levels. A randomized cross-over feeding trial among men of African ancestry showed that alcohol improves lipid profiles and reduces atherosclerosis-related inflammatory markers in plasma (36). Furthermore, sorghum-based beers are widely consumed in African populations and the high phenolic content of these drinks has been found to reduce the levels of leukocyte adhesion molecules and inflammatory biomarkers (10). Contrary to our findings the ARIC study observed that African Americans who are current drinkers were more likely to develop coronary heart disease than Europeans (37). These inconsistent findings further highlight the complex association of alcohol with CVDs and calls for more in-depth longitudinal, gene-alcohol interaction and genome wide association studies (GWAS) to provide further insight into the relationship of alcohol intake with atherosclerotic diseases in African populations.

Africans showed the weakest positive association between LDL-C, and one of the strongest negative associations between $\mathrm{HDL}-\mathrm{C}$ and CIMT compared to the other race-ethnic groups. Previous studies had demonstrated that African populations have lower lipid levels (especially LDL-C and isolated HDL-C) and this may have resulted in a weaker association with CVD morbidity and mortality compared to other race-ethnic groups $(38,39)$. Low LDL-C and weak association of LDL with CIMT may be a driver of the lower level of atherosclerosis in African populations but the effect of $\mathrm{HDL}-\mathrm{C}$ on CVD risk is uncertain. Lifestyle differences between various race-ethnic groups may explain some of the observed differences in both the risk factors for CVD and the CIMT measurements.

The raw CIMT levels were higher in men compared to women for all cohorts except those from Africa, where CIMT was similar across genders. Gender 
differences in CIMT may be related to the relative exposure to CVD risk factors (40) and when CIMT values were adjusted for these risk factors their levels were found to be higher in men than women within the African race-ethnic group and in all the other groups.

Our study has demonstrated that race-ethnic variations exist in the association of classical cardiovascular risk factors with CIMT. These observed differences may result from a complex gene-environment and other environmental factors and highlight the importance of further research to elucidate the mechanisms involved in the differential association of CVD risk factors with sub-clinical atherosclerosis across different population groups.

Strengths and limitations: In these IPD-MAs, we pooled data from 34,025 adults between the ages of 40 and 60 years from 15 cohorts drawn from Africa, Asia, Europe and North America. This is the first study to compare the association of CVD risk factors with CIMT in a large population of Africans residing in Eastern, Western and Southern Africa with that in other large cohorts drawn from different ethnic groups. The addition of the African population to the meta-analysis considerably expands our understanding of race-ethnicity and atherosclerotic cardiovascular disease. Using an IPD approach makes this study robust since we have been able to make direct comparison across the various ethnic groups. Harmonization of the outcome measure and the variables used in these analyses limited bias. The use of adjusted multilevel mixed-effect analyses to determine the mean differences in CIMT makes our results reliable and comparable across race-ethnic groups. We also acknowledge that due to the relatively small number of Asian and Hispanic subjects included in this study, our results may not be generalizable to these ethnicities as a whole. Furthermore, the Hispanic population and a large majority of the Asian population were resident in the USA and therefore any data should not be extrapolated to such race-ethnic groups resident outside of the USA.

Adjustment of the mean CIMT levels for the effect of established CVD risk factor enabled us to determine whether observed variations in mean CIMT across race-ethnic groups were due to differential patterning of CVD risk factors. We were also able to account for across and within ethnic group differences conferred by the different cohorts included in the IPD while preserving the original design and composition of the cohort. The H3Africa AWI-Gen study was conducted recently (2013 to 2015) while studies in the USE-IMT cohorts were mainly conducted between 1990 and 2000. This however should not affect the observed relationships since evidence from the Framingham study demonstrates significant relations that were observed in the baseline cohort 73 years ago still exist (41). We do acknowledge that residual confounding from 
some unmeasured factors may contribute to some of the observed ethnic differences.

A limitation of any meta-analysis is that analyses can only be performed on variables that are common to all the studies. In the present meta-analysis, dietary intake data was not available across all the studies and this is a limitation since previous investigations have shown associations between nutrient intake and CIMT. Thus, a recent meta-analysis of clinical trials focusing on the reduction of CIMT demonstrated that dietary interventions significantly slowed CIMT progression (42). In addition, we did not have medication use for all the studies.

A recent review by Mitchell et al (2021) reported that over time changes in technology have improved image resolutions of CIMT measurements (43). This may therefore affect cross cohort comparisons due to differences in the ultrasound technologies used. We believe however that the use of z-scores may offer a measure of standardization to overcome this problem and that the observed associations of CIMT with CVD risk factors will be minimally affected.

\section{Conclusion}

The differences in the magnitude of the associations of CVD risk factors with CIMT has implications for ethnic specific primary prevention strategies and also offer insights into ethnic-specific mechanisms involved in the aetiology of CVD. The current study does suggest that high CIMT levels in African Americans could be the result of genetics, with alleles predisposing to higher CIMT levels possibly originating in West Africa. However, it cannot be excluded that lifestyle factors, such as diet, may also contribute to these differences in CIMT levels and that gene-environment interactions may also be involved.

\section{Author contribution}

E.A.N., M.L.B., K.K.G., and N.J.C. conceived the paper and designed the data analyses plan. E.A.N. analyzed the data and wrote the first draft with contributions from K.K.G., N.J.C., and M.L.B. E.A.N., G. Agongo, G. Asiki, P.R.B., S.S.R.C., N.J.C., L.K.M., A.R.O., S.M.T., and M.R. are responsible for AWI-Gen study data collection and quality. T.J.A., D.J.C., G.E., J. de Graaf., M.K., J.K., E.M.L., E.B.M., S.O., J.F.P., T.R., J.T.S., T.P.T., D.E.G., and M.L.B. are responsible for data of the USE-IMT cohorts. All authors critically reviewed and approved the final draft for submission

\section{Acknowledgements}

We acknowledge the generosity of participants in the various cohorts who volunteered to participant in the studies. We also acknowledge the contributions 
of staff of the various cohorts who worked tirelessly to get credible data. Investigators responsible for the conception and design of the original AWIGen study include: Michèle Ramsay (PI), Osman Sankoh), Alisha Wade, Stephen Tollman, Kathleen Kahn, Marianne Alberts, Catherine Kyobutungi, Halidou Tinto, Abraham Oduro, Shane Norris, Scott Hazelhurst, Nigel Crowther, Himla Soodyall and Zane Lombard. Investigators responsible for the cohort from the USE-IMT consortium include: Michiel L. Bots (PI), David Couper, Gunnar Engström, Gregory W. Evans, Jacqueline de Graaf, Diederick E. Grobbee (co-PI), Maryam Kavousi (co-PI), Ellisiv B. Mathiessen, Tatjana Rundek, Tomi-Pekka Tuomainen, Todd J. Anderson, Joseph F. Polak, Jussi Kauhanen, Shuhei Okazaki, Akihiko Kitamura, Jukka T. Salonen and Eva M. Lonn.

\section{Sources of Funding}

EAN is supported by a grant from the Global Health Support Program of the University Medical Center Utrecht (UMCU), Utrecht University, The Netherlands and the Navrongo Health Research Centre (NHRC), Ghana. The AWI-Gen Collaborative Centre is funded by the National Human Genome Research Institute (NHGRI), Office of the Director (OD), Eunice Kennedy Shriver National Institute Of Child Health \& Human Development (NICHD), the National Institute of Environmental Health Sciences (NIEHS), the Office of AIDS research (OAR) and the National Institute of Diabetes and Digestive and Kidney Diseases (NIDDK), of the National Institutes of Health (NIH) under award number U54HG006938 and its supplements, as part of the H3Africa Consortium. Additional funding was leveraged from the Department of Science and Technology, South Africa, award number DST/CON 0056/2014. The USE-IMT project is supported by a grant from the Netherlands Organisation for Health Research and Development (ZonMw 200320003). The KIHD cohort was funded by grants from the Academy of Finland to Professor Jukka T. Salonen and from the NIH to Professor George A. Kaplan. The ARIC study is funded in whole or in part with federal funds from the National Heart, Lung, and Blood Institute, National Institutes of Health, Department of Health and Human Services under contract numbers HHSN2682017000011, HHSN268201700002I, HHSN268201700003I, HHSN268201700005I and HHSN268201700004I. The MESA study is sponsored by the National Heart, Lung and Blood Institute of the National Institute of Health. The authors thank the staff and participants of the ARIC and MESA studies for their important contributions. The views expressed in this manuscript are those of the authors and do not necessarily represent the views of the National Heart, Lung, and Blood Institute; the National Institutes of Health or the U.S. Department of Health and Human Services or the views of the other funders. The funders had no role in the design and conduct of the study; in the data collection, analysis, and interpretation of the data; or in the preparation, review, or approval of the manuscript. 


\section{Disclosures}

None 


\section{References}

1. GBD 2015 Risk Factors Collaborators. Global, regional, and national comparative risk assessment of 79 behavioural, environmental and occupational, and metabolic risks or clusters of risks, 1990-2015: a systematic analysis for the Global Burden of Disease Study 2015. Lancet. 2016;388(10053):1659-724.

2. Yuyun MF, Sliwa K, Kengne AP, Mocumbi AO, Bukhman G. Cardiovascular Diseases in Sub-Saharan Africa Compared to High-Income Countries: An Epidemiological Perspective. Global Heart. 2020;15(1).

3. Winham SJ, de Andrade M, Miller VM. Genetics of cardiovascular disease: Importance of sex and ethnicity. Atherosclerosis. 2015;241(1):219-28.

4. Sacco RL, Roberts JK, Boden-Albala B, Gu Q, Lin IF, Kargman DE, et al. Race-ethnicity and determinants of carotid atherosclerosis in a multiethnic population. The Northern Manhattan Stroke Study. Stroke. 1997;28(5):929-35.

5. Budoff MJ, Yang TP, Shavelle RM, Lamont DH, Brundage BH. Ethnic differences in coronary atherosclerosis. Journal of the American College of Cardiology. 2002;39(3):408-12.

6. Gijsberts CM, Groenewegen KA, Hoefer IE, Eijkemans MJ, Asselbergs FW, Anderson $\mathrm{TJ}$, et al. Race/Ethnic Differences in the Associations of the Framingham Risk Factors with Carotid IMT and Cardiovascular Events. PLoS One. 2015;10(7):e0132321.

7. Yusuf S, Hawken S, Ôunpuu S, Dans T, Avezum A, Lanas F, et al. Effect of potentially modifiable risk factors associated with myocardial infarction in 52 countries (the INTERHEART study): case-control study. The Lancet. 2004;364(9438):937-52.

8. Yusuf $S$, Joseph P, Rangarajan S, Islam S, Mente A, Hystad P, et al. Modifiable risk factors, cardiovascular disease, and mortality in 155722 individuals from 21 highincome, middle-income, and low-income countries (PURE): a prospective cohort study. The Lancet. 2020;395(10226):795-808.

9. Bots ML, Evans GW, Tegeler CH, Meijer R. Carotid Intima-media Thickness Measurements: Relations with Atherosclerosis, Risk of Cardiovascular Disease and Application in Randomized Controlled Trials. Chin Med J 2016;129(2):215-26.

10. Nonterah EA, Boua PR, Klipstein-Grobusch K, Asiki G, Micklesfield LK, Agongo G, et al. Classical cardiovascular risk factors and HIV are associated with carotid intimamedia thickness in adults from sub-Saharan Africa: Findings from H3Africa AWI-Gen Study. J Am Heart Assoc. 2019;8(14).

11. Den Ruijter HM, Peters SAE, Anderson TJ, Britton AR, Dekker JM, Eijkemans MJ, et al. Common Carotid Intima-Media Thickness Measurements in Cardiovascular Risk Prediction: A Meta-analysis. JAMA. 2012;308(8):796-803.

12. Ali SA, Soo C, Agongo G, Alberts M, Amenga-Etego L, Boua RP, et al. Genomic and environmental risk factors for cardiometabolic diseases in Africa: methods used for Phase 1 of the AWI-Gen population cross-sectional study. Glob Health Action. 2018;11(sup2):1507133. 
13. Anderson TJ, Charbonneau F, Title LM, Buithieu J, Rose MS, Conradson $\mathrm{H}$, et al. Microvascular function predicts cardiovascular events in primary prevention: longterm results from the Firefighters and Their Endothelium (FATE) study. Circulation. 2011;123(2):163-9.

14. Li R, Duncan BB, Metcalf PA, Crouse III JR, A R Sharrett, H A Tyroler, et al. B-modedetected carotid artery plaque in a general population. Atherosclerosis Risk in Communities (ARIC) Study Investigators. Stroke. 1994;25(12):2377-83.

15. Mora S, Szklo M, Otvos JD, Greenland P, Psaty BM, Goff DC, Jr., et al. LDL particle subclasses, LDL particle size, and carotid atherosclerosis in the Multi-Ethnic Study of Atherosclerosis (MESA). Atherosclerosis. 2007;192(1):211-7.

16. Prabhakaran S, Singh $R$, Zhou $X$, Ramas $R$, Sacco RL, Rundek T. Presence of calcified carotid plaque predicts vascular events: the Northern Manhattan Study. Atherosclerosis. 2007;195(1):e197-201.

17. Salonen R, Salonen JT. Determinants of carotid intima-media thickness: a populationbased ultrasonography study in eastern Finnish men. J Intern Med. 1991;229(3):225-31.

18. Rosvall M, Östergren PO, Hedblad B, Isacsson SO, Janzon L, Berglund G. Occupational status, educational level, and the prevalence of carotid atherosclerosis in a general population sample of middle-aged Swedish men and women. Results from the Malmö Diet and Cancer Study. Am J Epidemiol. 2000;152.

19. Stensland-Bugge E, Bønaa KH, Joakimsen O, Njølstad I. Sex Differences in the Relationship of Risk Factors to Subclinical Carotid Atherosclerosis Measured 15 Years Later The Tromsø Study. Stroke. 2000;31(3):574-81.

20. Holewijn S, den Heijer M, Swinkels DW, Stalenhoef AF, de Graaf J. The metabolic syndrome and its traits as risk factors for subclinical atherosclerosis. J Clin Endocrinol Metab. 2009;94(8):2893-9.

21. Kitagawa K, Hougaku H, Yamagami H, Hashimoto H, Itoh T, Shimizu Y, et al. Carotid intima-media thickness and risk of cardiovascular events in high-risk patients. Results of the Osaka Follow-Up Study for Carotid Atherosclerosis 2 (OSACA2 Study). Cerebrovasc Dis. 2007;24(1):35-42.

22. Kaplan JB, Bennett T. Use of Race and Ethnicity in Biomedical Publication. JAMA. 2003;289(20):2709-16.

23. Phinney JS, Ong AD. Conceptualization and measurement of ethnic identity: Current status and future directions. Journal of Counseling Psychology. 2007;54(3):271-81.

24. Ross PT, Hart-Johnson T, Santen SA, Zaidi NLB. Considerations for using race and ethnicity as quantitative variables in medical education research. Perspect Med Educ. 2020;9(5):318-23.

25. So-Armah K, Benjamin LA, Bloomfield GS, Feinstein MJ, Hsue P, Njuguna B, et al. HIV and cardiovascular disease. The Lancet HIV. 2020;7(4):e279-e93.

26. Mach F, Baigent C, Catapano AL, Koskinas KC, Casula M, Badimon L, et al. 2019 ESC/ EAS Guidelines for the management of dyslipidaemias: lipid modification to reduce cardiovascular risk. Eur Heart J. 2020;41(1):111-88. 
27. Grundy SM, Stone NJ, Bailey AL, Beam C, Birtcher KK, Blumenthal RS, et al. 2018 AHA/ ACC/AACVPR/AAPA/ABC/ACPM/ADA/AGS/APhA/ASPC/NLA/PCNA Guideline on the Management of Blood Cholesterol: A Report of the American College of Cardiology/ American Heart Association Task Force on Clinical Practice Guidelines. Circulation. 2019;139(25):e1082-e143.

28. Amarenco P, Kim JS, Labreuche J, Charles H, Abtan J, Bejot $Y$, et al. A Comparison of Two LDL Cholesterol Targets after Ischemic Stroke. N Engl J Med. 2020;382(1):9.

29. Koch S, Nelson D, Rundek T, Mandrekar J, Rabinstein A. Race-ethnic variation in carotid bifurcation geometry. J Stroke Cerebrovasc Dis. 2009;18(5):349-53.

30. Zakharia F, Basu A, Absher D, Assimes TL, Go AS, Hlatky MA, et al. Characterizing the admixed African ancestry of African Americans. Genome Biol. 2009;10(12):R141.

31. Micheletti SJ, Bryc K, Ancona Esselmann SG, Freyman WA, Moreno ME, Poznik GD, et al. Genetic Consequences of the Transatlantic Slave Trade in the Americas. Am J Hum Genet. 2020;107(2):265-77.

32. Markert MS, Della-Morte D, Cabral D, Roberts EL, Jr., Gardener H, Dong C, et al. Ethnic differences in carotid artery diameter and stiffness: the Northern Manhattan Study. Atherosclerosis. 2011;219(2):827-32.

33. Ferreira JP, Girerd N, Bozec E, Machu JL, Boivin JM, London GM, et al. Intima-Media Thickness Is Linearly and Continuously Associated With Systolic Blood Pressure in a Population-Based Cohort (STANISLAS Cohort Study). J Am Heart Assoc. 2016;5(6).

34. Bierut LJ, Goate AM, Breslau N, Johnson EO, Bertelsen S, Fox L, et al. ADHIB is associated with alcohol dependence and alcohol consumption in populations of European and African ancestry. Mol Psychiatry. 2012;17(4):445-50.

35. Crabb DW, Matsumoto M, Chang D, You M. Overview of the role of alcohol dehydrogenase and aldehyde dehydrogenase and their variants in the genesis of alcohol-related pathology. Proc Nutr Soc. 2004;63(1):49-63.

36. Chiva-Blanch G, Magraner E, Condines X, Valderas-Martinez P, Roth I, Arranz S, et al. Effects of alcohol and polyphenols from beer on atherosclerotic biomarkers in high cardiovascular risk men: a randomized feeding trial. Nutr Metab Cardiovasc Dis. 2015;25(1):36-45.

37. Fuchs FD, Chambless LE, Folsom AR, Eigenbrodt ML, Duncan BB, Gilbert A, et al. Association between alcoholic beverage consumption and incidence of coronary heart disease in whites and blacks: the Atherosclerosis Risk in Communities Study. Am J Epidemiol. 2004;160(5):466-74.

38. Bentley AR, Rotimi CN. Interethnic Differences in Serum Lipids and Implications for Cardiometabolic Disease Risk in African Ancestry Populations. Glob Heart. 2017;12(2):141-50.

39. Bartlett J, Predazzi IM, Williams SM, Bush WS, Kim Y, Havas S, et al. Is Isolated Low HDL-C a CVD Risk Factor?: New Insights from the Framingham Offspring Study. Circulation Cardiovascular quality and outcomes. 2016;9(3):206-12

40. Yusuf S, Reddy S, Ôunpuu S, Anand S. Global Burden of Cardiovascular Diseases. Part I: General Considerations, the Epidemiologic Transition, Risk Factors, and Impact of Urbanization. Circulation. 2001;104(22):2746-53. 
41. Hajar R. Framingham Contribution to Cardiovascular Disease. Heart Views. 2016;17(2):78-81.

42. Willeit P, Tschiderer L, Allara E, Reuber K, Seekircher L, Gao L, et al. Carotid Intima-Media Thickness Progression as Surrogate Marker for Cardiovascular Risk: Meta-Analysis of 119 Clinical Trials Involving 100667 Patients. Circulation. 2020;142(7):621-42.

43. Mitchell C, Korcarz CE, Zagzebski JA, Stein JH. Effects of ultrasound technology advances on measurement of carotid intima-media thickness: A review. Vasc Med. 2021;26(1):81-5. 


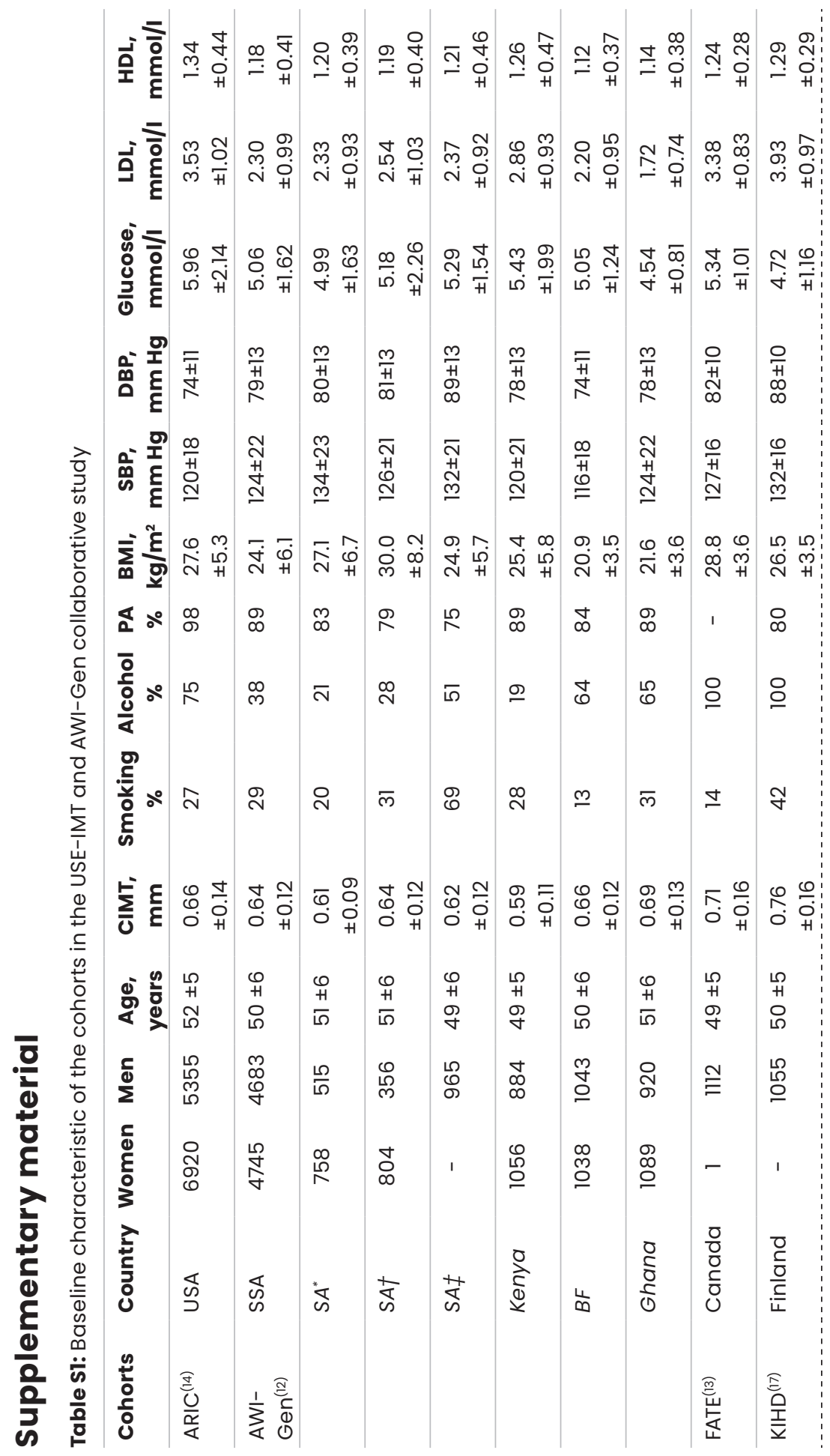




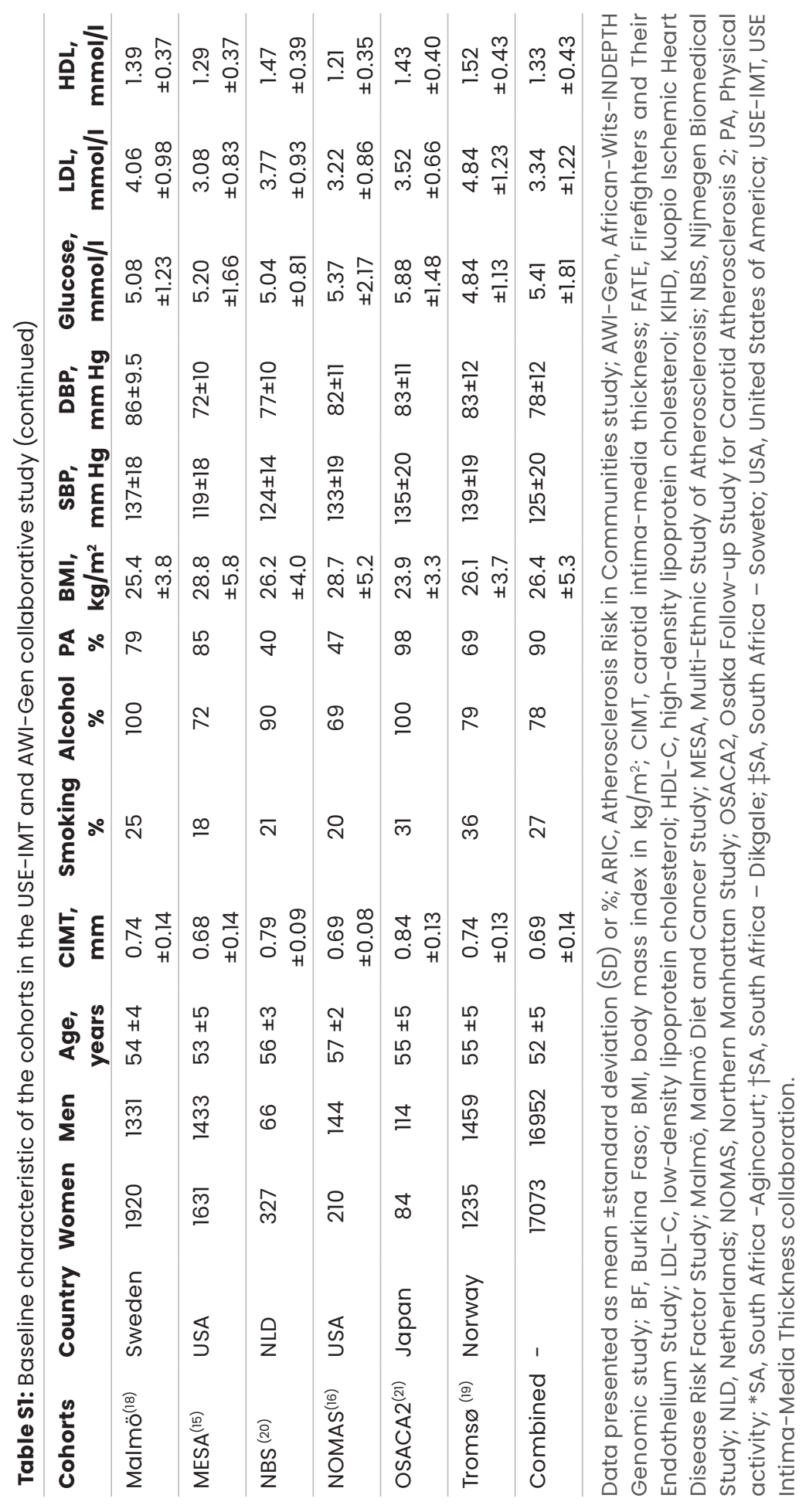




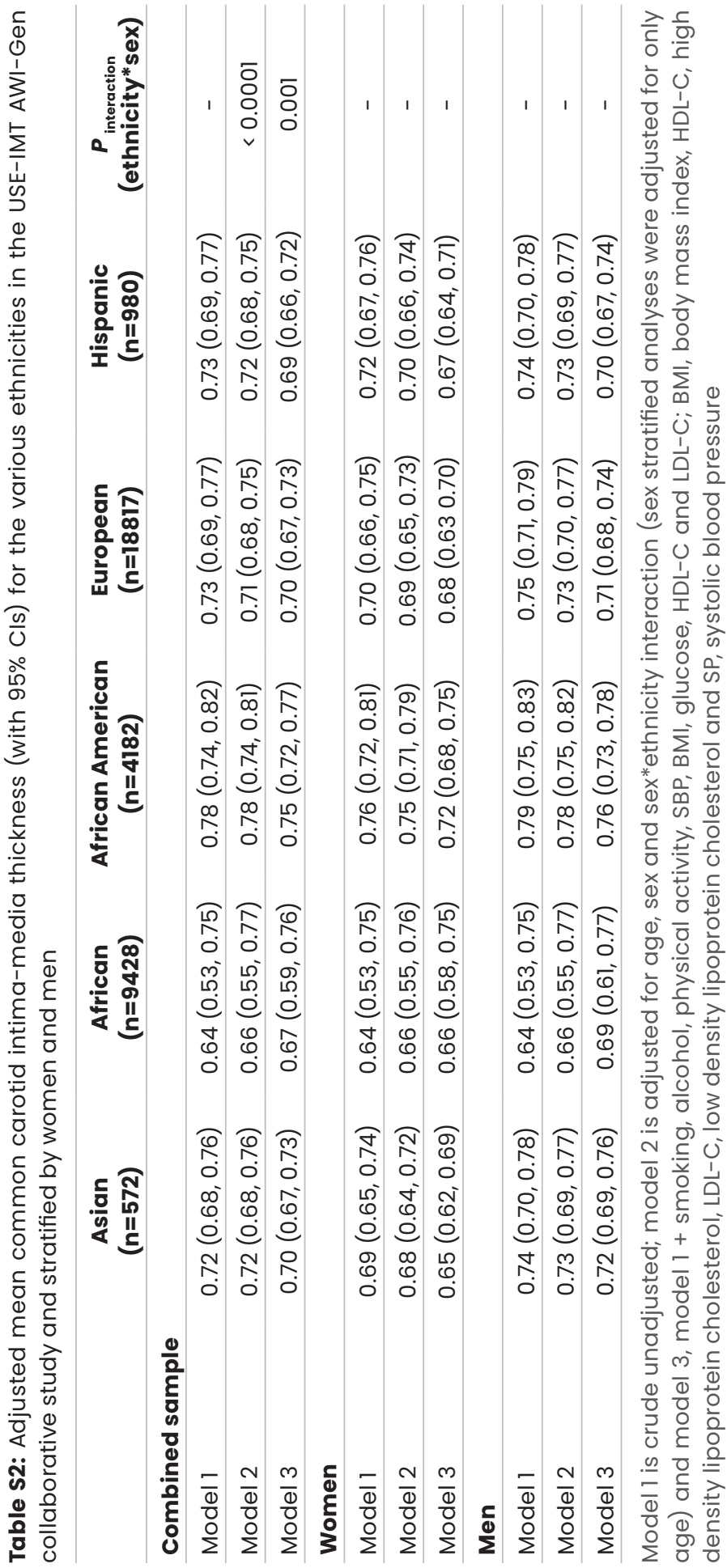



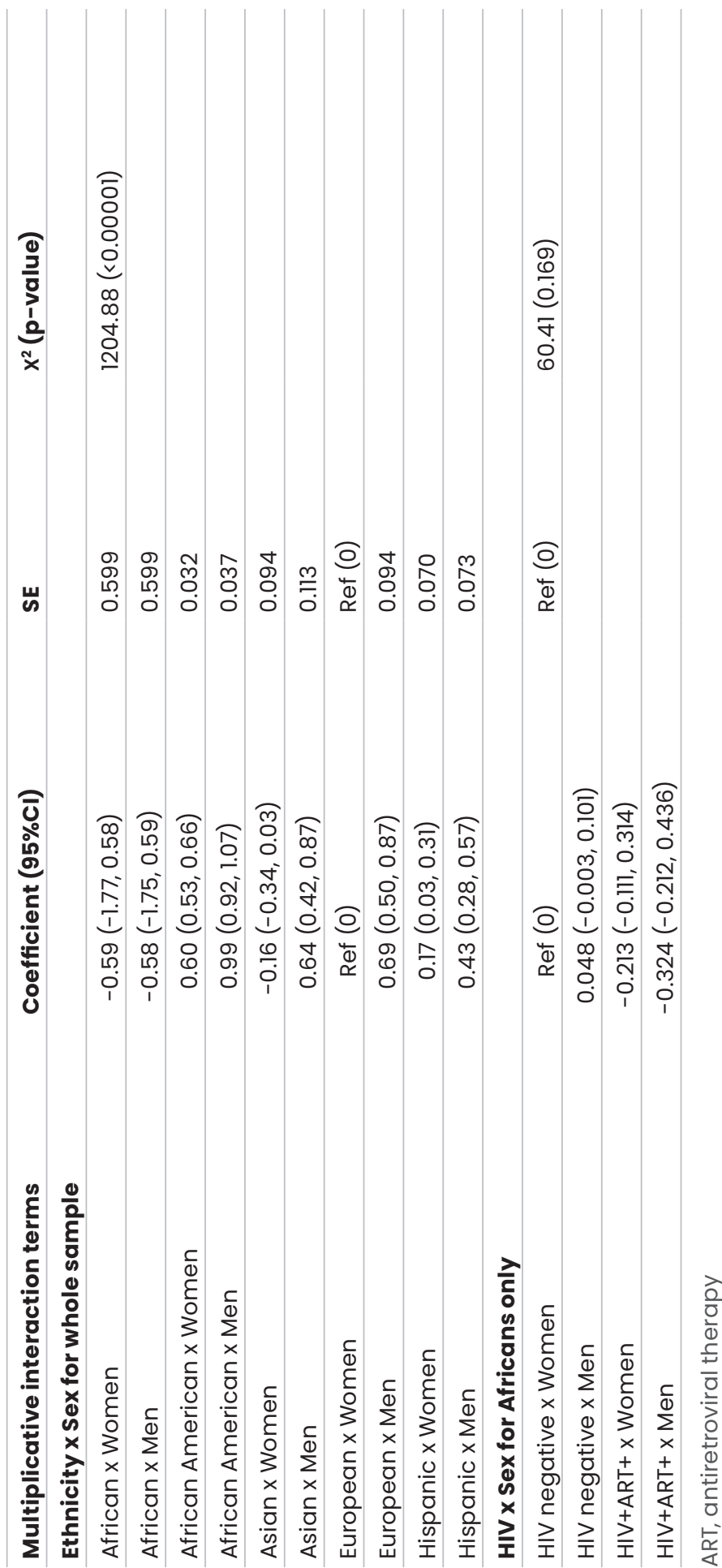


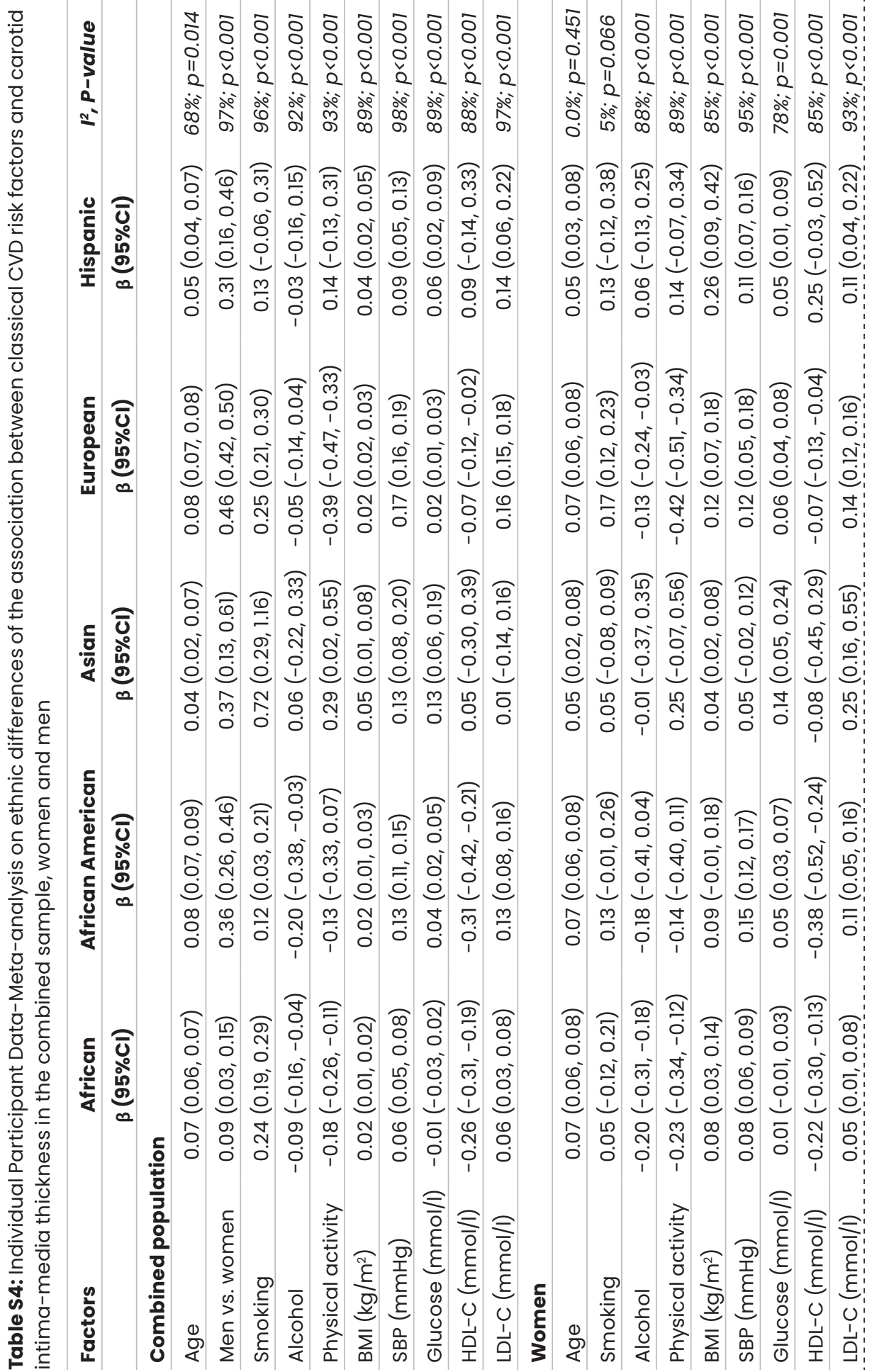




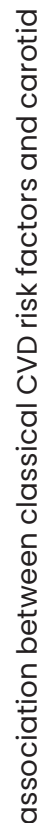

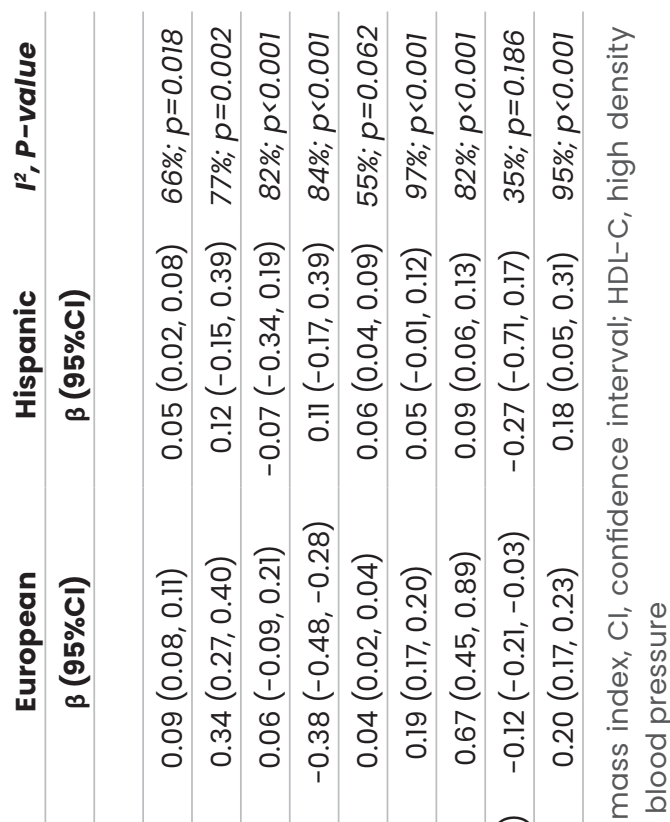

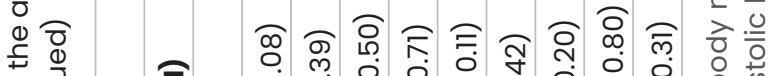

诃

क)

○

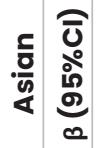

O.

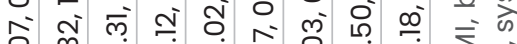

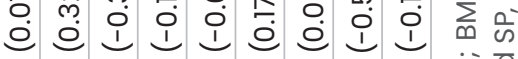

$\frac{1}{(1)} \subset$

$\infty$

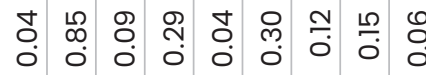

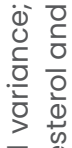

음

芒

ㅇ

. 3

要

증

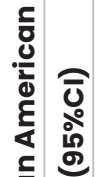

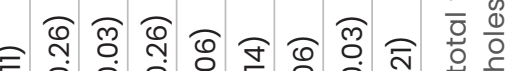

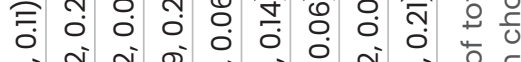

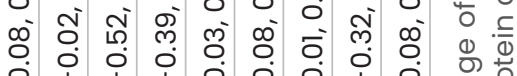

过 1 i i

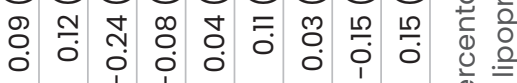

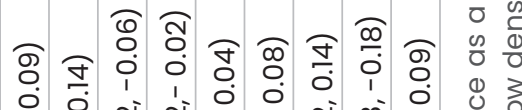

일

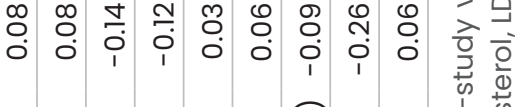


Table S5: Comparing the variance explained by modeling the association of LDL, HDL and LDL/HDL ratio with common carotid intima-media thickness

\begin{tabular}{lllll}
\hline & $\begin{array}{l}\text { Variance } \\
\text { inflation } \\
\text { factor for } \\
\text { model } 1\end{array}$ & $\mathbf{R}^{2}$ for model 2 & $\mathbf{R}^{2}$ for model 3 & Comment \\
\hline African ancestry & 7.32 & 0.18 & 0.18 & No change in $\mathrm{R}^{2}$ \\
\hline African American & 8.29 & 0.17 & 0.17 & No change in $\mathrm{R}^{2}$ \\
\hline Asians & 12.6 & 0.26 & 0.26 & No change in $\mathrm{R}^{2}$ \\
\hline European ancestry & 8.41 & 0.16 & 0.16 & No change in $\mathrm{R}^{2}$ \\
\hline Hispanic & 10.04 & 0.24 & 0.24 & No change in $\mathrm{R}^{2}$ \\
\hline
\end{tabular}

Model 1 included the following independent variables: age, sex, smoking, physical activity, alcohol, BMI, SBP, Glucose, HDL-C, LDL-C and LDL-C/HDL-C ratio; Model 2 included age, sex, smoking, physical activity, alcohol, BMI, SBP, Glucose, LDL-C and HDL-C and Model 3 included age, sex, smoking, physical activity, alcohol, BMI, SBP, Glucose, LDL-C/ $\mathrm{HDL}-\mathrm{C}$ ratio 


\section{Part 2:}

\section{CIMT and cardiovascular disease risk}




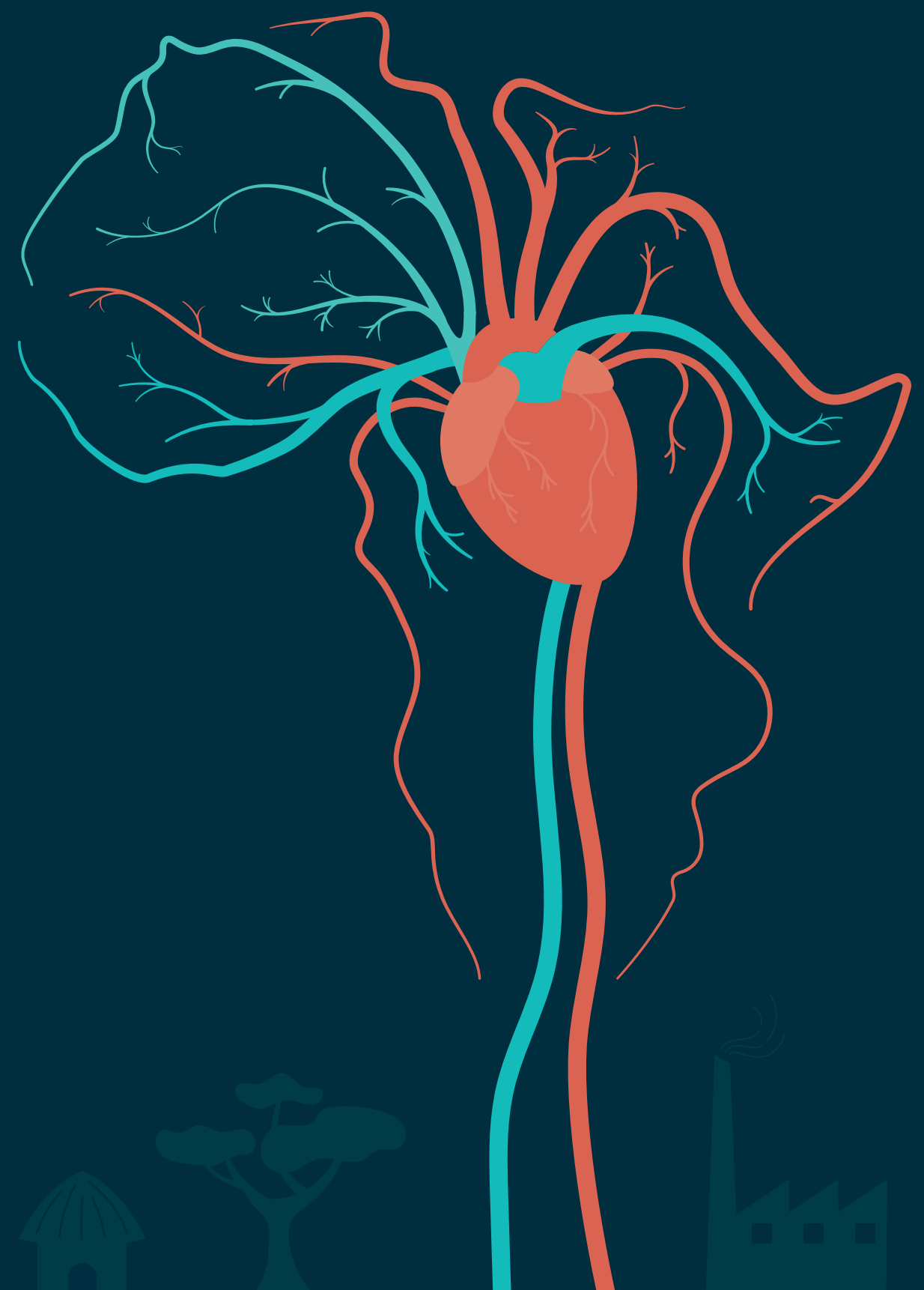




\section{Abstract}

Background: The cardiovascular health index (CVHI) introduced by the American Heart Association is a valid, accessible, simple, and translatable metric for monitoring cardiovascular health in a population. Components of the $\mathrm{CVHI}$ include the following seven cardiovascular risk factors (often captured as life's simple 7): smoking, dietary intake, physical activity, body mass index, blood pressure, glucose, and total cholesterol. We sought to expand the evidence for its utility to under-studied populations in sub-Saharan Africa, by determining its association with common carotid intima-media thickness (CIMT).

Methods: We conducted a cross-sectional study involving 9011 participants drawn from Burkina Faso, Ghana, Kenya, and South Africa. We assessed established classical cardiovascular risk factors and measured carotid intimamedia thickness of the left and right common carotid arteries using B-mode ultrasonography. Adjusted multilevel mixed-effect linear regression was used to determine the association of CVHI with common CIMT. In the combined population, an individual participant data meta-analyses random-effect was used to conduct pooled comparative sub-group analyses for differences between countries, sex, and socio-economic status.

Results: The mean age of the study population was $51 \pm 7$ years and $51 \%$ were women, with a mean common CIMT of $637 \pm 117 \mu \mathrm{m}$ and CVHI score of $10.3 \pm 2.0$. Inverse associations were found between CVHI and common CIMT ( $\beta$-coefficients [95\% confidence interval]: Burkina Faso, - $6.51[-9.83,-3.20] \mu \mathrm{m}$; Ghana, - $5.42[-8.90,-1.95]$; Kenya, - 6.58 [- 9.05, - 4.10]; and South Africa, - 7.85 $[-9.65,-6.05])$. Inverse relations were observed for women $(-4.44[-6.23,-$ $2.65])$ and men $(-6.27[-7.91,-4.64])$ in the pooled sample. Smoking $(p<0.001)$, physical activity $(p<0.001)$, and hyperglycemia $(p<0.001)$ were related to CIMT in women only, while blood pressure and obesity were related to CIMT in both women and men $(p<0.001)$.

Conclusion: This large pan-African population study demonstrates that CVHI is a strong marker of subclinical atherosclerosis, measured by common CIMT and importantly demonstrates that primary prevention of atherosclerotic cardiovascular disease in this understudied population should target physical activity, smoking, obesity, hypertension, and hyperglycemia.

Keywords: Cardiovascular health index, Carotid intima-media thickness, Subclinical atherosclerosis, Sub-Saharan Africa, Primary prevention, Cardiovascular diseases, Screening, Understudied populations 


\section{Background}

Cardiovascular disease (CVD) is a leading cause of global deaths $(1,2)$ raising huge public health concerns. Sub-Saharan Africa (SSA) is experiencing a rising burden of non-communicable diseases including CVD (3), alongside a reduction in the prevalence of infectious diseases (4). Increasing urbanization, changing lifestyles and population ageing is probably accounting for the surge in the prevalence of CVD in SSA $(3,4)$. This presents a challenge to the health systems of African countries and requires a pragmatic approach to the prevention of CVD. Where risk equations such as Framingham (5) and Pooled Cohort Equations (PCE) (6) have served as valuable tools in assessing the risk of CVD globally, these have inherent limitations especially when applied to an African population (7). The American Heart Association and the American Stroke Association (AHA/ASA) developed the Cardiovascular Health Index (CVHI) as a new public health metric for assessing and monitoring cardiovascular health (8-11). The components of the CVHI often referred to as life's simple seven include smoking, dietary intake, physical activity, body mass index (BMI), blood pressure, glucose and total cholesterol.

Due to the lack of data on incident CVD events, carotid-intima media thickness (CIMT) is often used as an alternate marker of CVD risk. Increased CIMT has been shown to be related to several risk factors for CVD and CVD risk globally $(12,13)$ including SSA populations (14).

The CVHI metric is proven to be related to CIMT, a marker of subclinical atherosclerosis (15), and coronary artery calcification (16) in both Caucasian and Asian populations (17). In Africa, the association of CVHI with subclinical atherosclerosis has only been reported in a relatively small study of HIV-positive $(n=105)$ and non-infected $(n=100)$ individuals (18) in Uganda and recently in a rural setting in Limpopo, South Africa (19). Expanding the evidence to a larger pan-African population would help to clarify whether the CVHI can be used as a simple screening tool for cardiovascular health within the existing health systems of resource-constrained SSA countries. Therefore, we set out to assess the association between cardiovascular health, using $\mathrm{CVHI}$, and subclinical atherosclerosis in a large understudied African population living in SSA. 


\section{Methods}

\section{Study design and population}

The H3Africa Africa-Wits-INDEPTH (www.indepthnetwork.org) partnership for genomic studies (AWI-Gen) project is a population-based cross-sectional study. Participants included older adult women and men aged 40-60 years drawn from six sites in four SSA countries $(20,21)$.

Five of the sites were INDEPTH-Network (International Network for the Demographic Evaluation of Populations and Their Health) Health and Demographic Surveillance Study (HDSS) sites namely Navrongo HDSS in Ghana, Nanoro HDSS in Burkina Faso, Nairobi urban HDSS in Kenya and Agincourt and DIMAMO HDSS's in South Africa. The sixth site was the South African MRC/ University of the Witwatersrand Developmental Pathways to Health Research Unit (DPHRU) in Soweto, South Africa. Participants were randomly sampled from existing sampling frames in the various recruitment sites. Those who reported history of cardiovascular diseases (myocardial infarction, stroke) and chronic kidney disease were excluded from the analyses. Pregnant women, closely related individuals (first degree relatives) and those residents in the communities for less than 10 years were excluded.

\section{Independent variable: Cardiovascular health index (CVHI)}

The components of the CVHI as defined by AHA/ASA include blood pressure, fasting glucose, total cholesterol, body mass index, physical activity, diet, and smoking (8). Each CVHI component was given a point score of 0,1 , or 2 to represent poor, intermediate and ideal health respectively. Details of how the categories were defined are presented in Additional file 1: Table S1. The AHA recommended cutoffs are consistent with recommendations from the World Health Organization $(22,23)$ and other organizations (24). These cutoffs have recently been used in a study in rural South Africa (19). An overall CVHI score was computed as the sum of all $7 \mathrm{CVHI}$ components, ranging from 0 to 14 . We stratified participants into 3 mutually exclusive categories based on overall CVHI score: Ideal cardiovascular health (iCVH) defined as 12-14 points, intermediate cardiovascular health (8-11 points), and poor cardiovascular health (0-7 points) (25). Details of how the various components of $\mathrm{CVHI}$ were measured and defined in the AWI-Gen study have been published elsewhere (21).

Briefly, the history of smoking tobacco products was self-reported and defined as never smoked, previous smoker and current smoker. Physical activity was measured as the level of moderate-to-vigorous physical activity (MVPA) from self-reported data on occupation, travel-related and leisure time physical activity. Diet was measured as the self-reported daily number of servings 
of fruits and vegetables. Weight was measured to the nearest $0.1 \mathrm{~kg}$ using a digital $200 \mathrm{~kg}$ capacity scale (Kendon Medical, South Africa). Standing height to the nearest millimeter was measured using a Harpenden digital stadiometer (Holtain, Wales). The BMI was then calculated as weight divided by height squared in $\mathrm{kg} / \mathrm{m}^{2}$. Blood pressure was measured from the left arm of the participant, seated with the arm at the level of the heart and having rested 5-10 minutes. Three readings were taken with the first value discarded and an average of the second and third computed as blood pressure. Venous blood was taken with the participant having fasted overnight and this sample was used to measure glucose and total cholesterol using colorimetric assays in a Randox Plus clinical chemistry analyser (Crumlin, UK). The coefficient of variation of the laboratory measurements for glucose was $2.3 \%$ and $1.5 \%$ for total cholesterol. All analyses were performed in a centralized laboratory at the DPHRU in Soweto according to good laboratory practice, and with external monitoring for quality control.

\section{Dependent variable: Common carotid atherosclerosis}

Carotid intima-media thickness was used as a marker of sub-clinical atherosclerosis. A carotid B-mode ultrasound scan (GE Healthcare, USA) with a 12L-RS straight probe was used to measure the CIMT of the right and left common carotid artery (CCA). Details of the measurement procedure used in this population are described elsewhere $(4,21)$. Briefly the right and left intima-media thickness of the common carotid arteries (CCA) were measured at a single angle of 45 degree tilt with the participant lying supine and neck supported with a pillow. A mean of the right and left common CIMT (in micrometers) was computed as the outcome variable.

\section{Potential confounders and effect modifiers}

Age was self-reported or calculated from reported date of birth while sex was reported at the time of recruitment. Household socio-economic status (SES) was determined based on household assets and quintiles calculated according to the practice implemented by the Demographic and Health Surveys (DHS) Program (http://www.dhsprogram.com/topics/wealth-index/Wealth-IndexConstruction.cfm). A principal component analysis of the listed household assets is conducted and predicting factor scores generated from these analyses. The predicted factor scores are then categorized into quintiles for each of the participating sites separately (21). Educational status was by asking the number years spent in school. Educational status was ascertained by asking the highest level of education (no formal education, primary, secondary or tertiary education). This was subsequently categorized as no formal education and yes formal education (primary, secondary and tertiary education). This took into consideration the number of years in each country at the different levels of 
school. HIV infection was self-reported in Burkina Faso and Ghana and testing was not offered due to the very low prevalence. Kenya and South Africa used locally available, government-approved rapid-test to conduct voluntary HIV rapid test. Antiretroviral therapy (ART) use was self-reported across all sites.

\section{Statistical analyses}

All data analyses were conducted using STATA version 14.2 (Statacorp, College Station, TX, USA). We used descriptive and inferential statistics to summarize our data. Using Epanechnikov kernel function in STATA, density curves were plotted to determine the distribution of the CVHI score and common CIMT for the various countries represented in the AWI-Gen study (Additional file 2: Figs S1 and S2). Continuous variables are reported as mean and standard deviation $( \pm S D)$ due to approximate normal distribution and categorical variables as sample number with respective percentages. The differences in prevalence of overall cardiovascular health between women and men were examined using Pearson's Chi squared test.

As the participants were resident in four different countries, we used multilevel mixed-effects generalized linear models to determine the associations of $\mathrm{CVHI}$ score with common CIMT. A variance co-variance method was used to obtain robust standard errors. Four progressively adjusted models were conducted as follows: model 1 was adjusted for age and sex; model 2 was adjusted for age, sex, level of education and household socio-economic status; in model 3, we adjusted for all the model 2 variables in addition to HIV and ART use. The fixed effects portion of the model was considered to vary according to country and hence country was specified as the random effect part of the mixed effect model. Separate models were fitted for the combined populations, women and men and countries.

The country specific models were fitted sequentially following the pattern explained above using adjusted linear regression models. Multilevel mixedeffects generalized linear models were conducted for South Africa which contained three different sites (site as the random effect). In the combined population, an individual participant data (IPD) meta-analyses was conducted. Pooled comparative sub-group analyses included comparison by country, sex and household SES. Our sub-group analyses included household SES since it has previously been linked to poor health outcomes (26) and atherosclerosis (27). Forest plots were subsequently plotted and weighted by sample size to avoid biased estimates (and for ease of comparison) and by default sample size instead of study weights appear on the plots. In addition line plots of the predicted linear association between $\mathrm{CVHI}$ score and CIMT were drawn and presented in supplementary material (Additional file 2: Figs S3 and S4). 
To determine the discriminatory power of each CVHI component metric to predict common CIMT, we generated dummy variables for the 3 levels of each CVHI metric using 'poor' as the reference. We then conducted adjusted multilevel mixed effects linear regression analyses of the dummy variables, with adjustment for age, sex, educational status and household SES in the pooled sample and for women and men separately. A two-tailed $p<0.05$ was considered statistically significant in all inferential analyses.

\section{Results}

After excluding participants (7.5\%) with missing data, 9011 adults from Burkina Faso, Ghana, Kenya and South Africa were included in these analyses. Basic characteristics are presented in Table 1. Fifty one percent of the study population was women and the average age was $51 \pm 7$ years. Physical activity levels were high (higher mean moderate-to-vigorous physical activity - minutes/week) in the populations from the two rural sites of West Africa. The average fruit and vegetable intake in the combined $\mathrm{AWI}-$ Gen population was $3 \pm 2$ servings per day. Mean BMI, systolic and diastolic blood pressure were highest in South Africa and Kenya and lowest in Ghana and Burkina Faso (Table 1). 

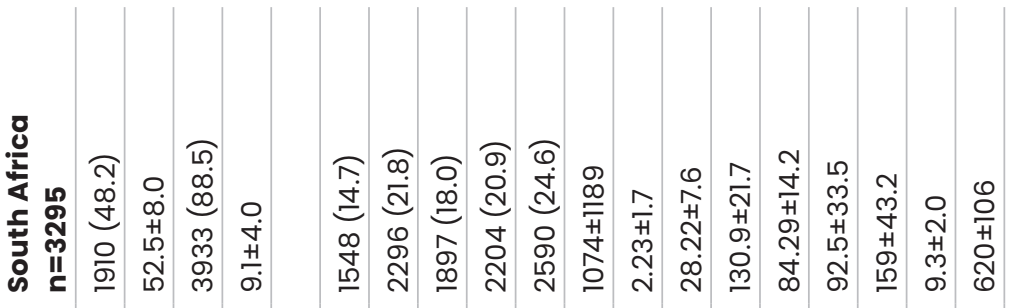

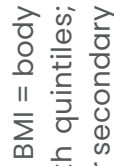

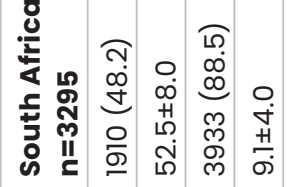

¿

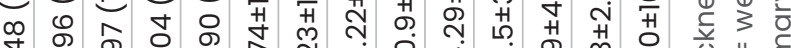

เ上 สำ

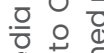

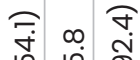

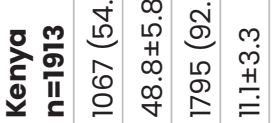

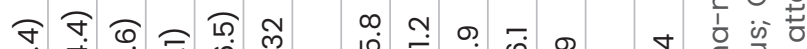

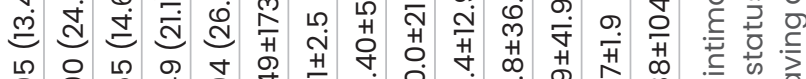

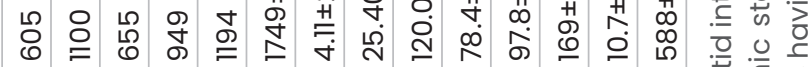

흘 ह

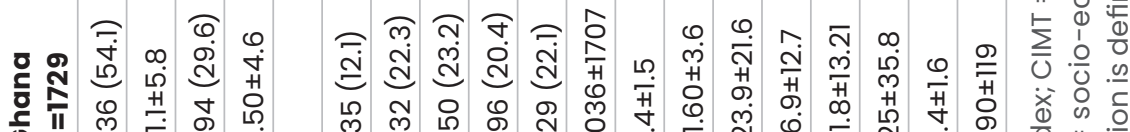

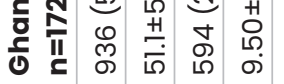

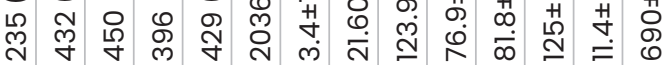

x क o

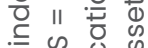

出

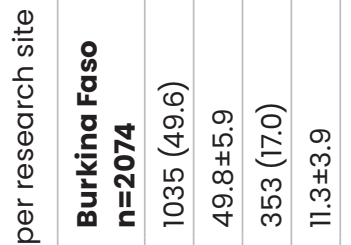

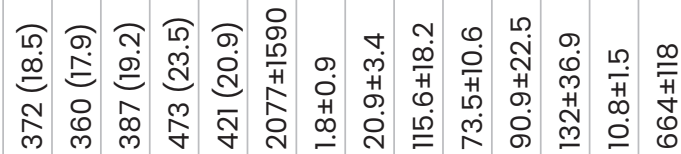

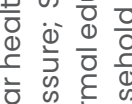

흘 ल

흔 운

ठ둥

.0ํำ

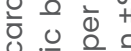

II

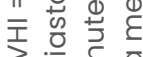

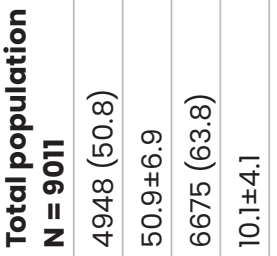

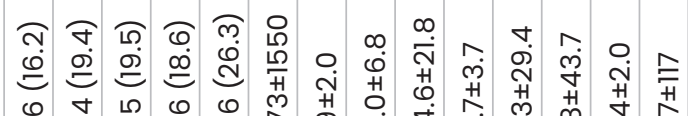

U

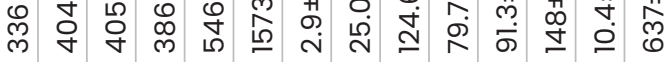

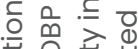

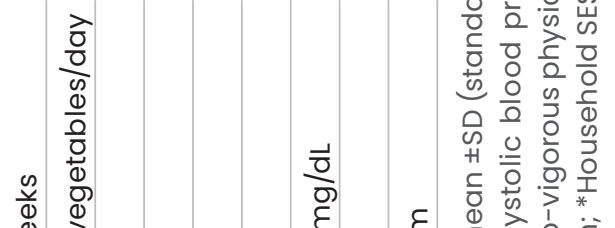

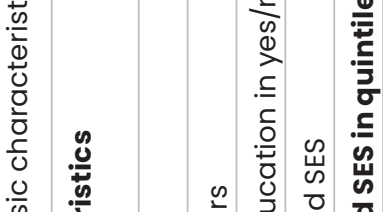

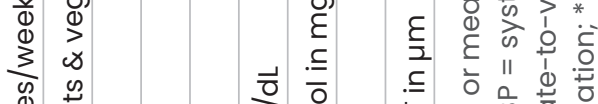

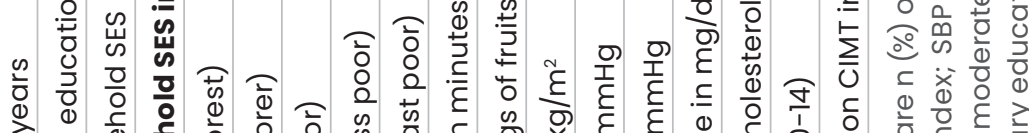

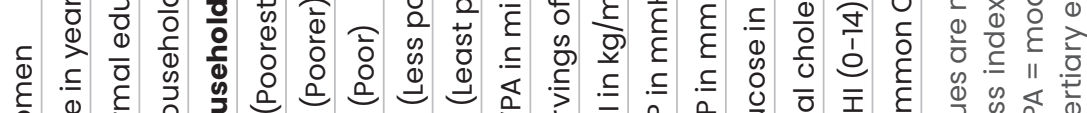

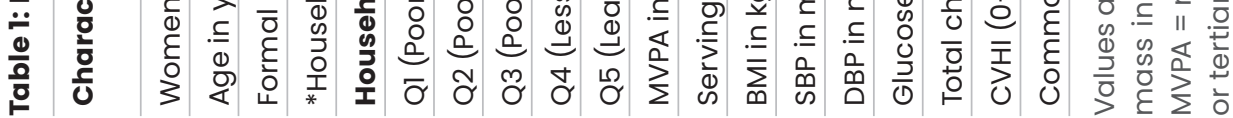


The prevalence of each of the 3 levels of cardiovascular health (poor, intermediate and ideal) for the total sample, the total sample stratified by sex, and the various countries is presented in Figure 1. In the combined sample iCVH was $31.9 \%$ with women (38.2\%) having a higher prevalence than men $(25.6 \%)$. Rural sites in Ghana (50.6\%) and Burkina Faso (44.3\%) had the highest prevalence of iCVH whilst the more urban sites in Kenya (37.4\%) and South Africa (11.3\%) had the lowest. The prevalence of the poor, intermediate and ideal levels of each of the $7 \mathrm{CVHI}$ components, according to sex and country, are presented in Table 2. The majority of participants were in the ideal category for total cholesterol, fasting glucose, physical activity, BMI and blood pressure with unhealthy diet and smoking placing the majority of participants in the poor category. Burkina Faso and Ghana had more participants in the ideal category for most CVHI components than South Africa and Kenya, except diet and physical activity where Burkina Faso had the least ideal levels compared to other countries.

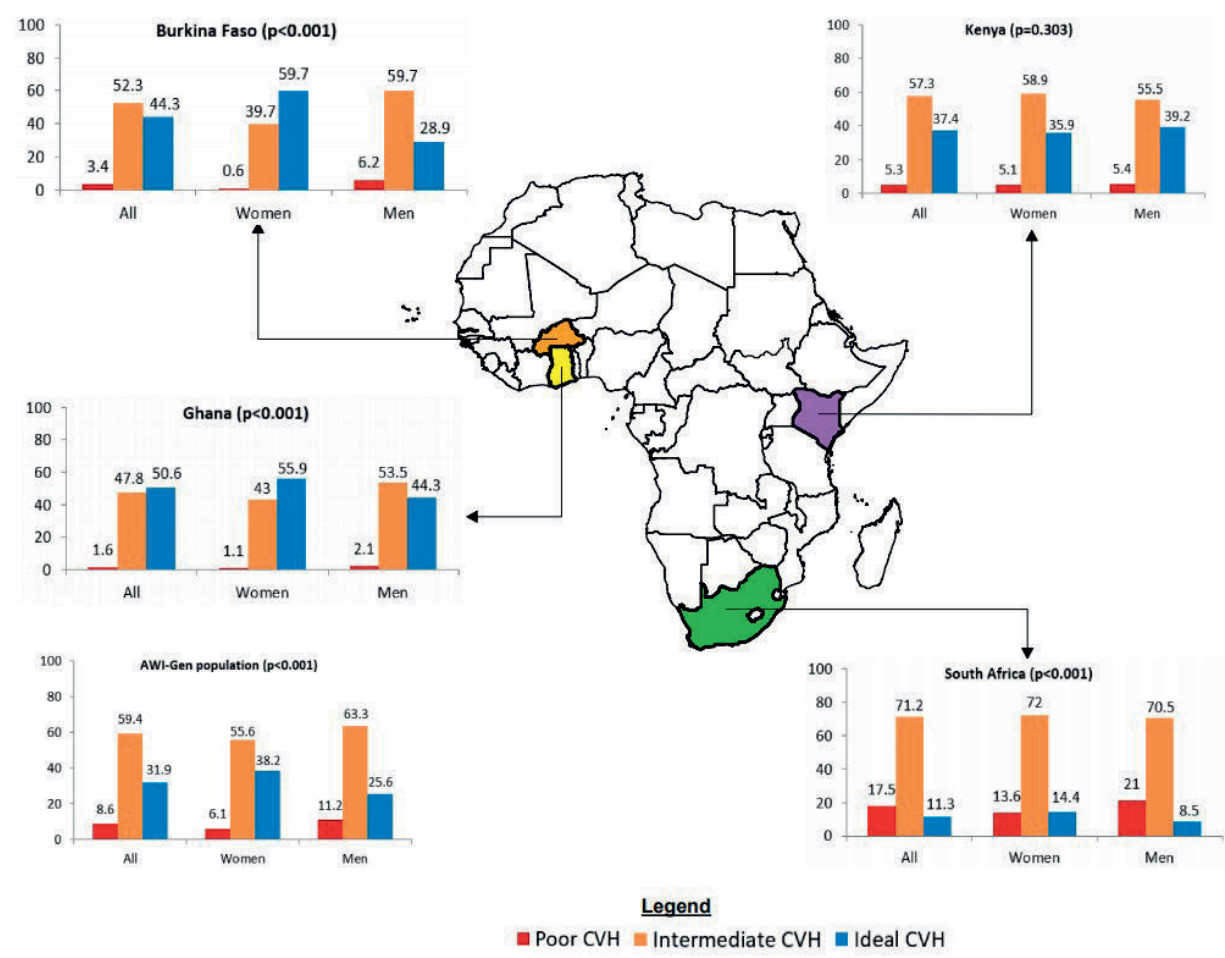

Figure 1: Map of Africa showing the prevalence of levels of cardiovascular health (CVH) for the various countries of the AWI-Gen study and according to sex, AWI-Gen population is the combined participant analysis. $P$-values denote differences between women and men. 


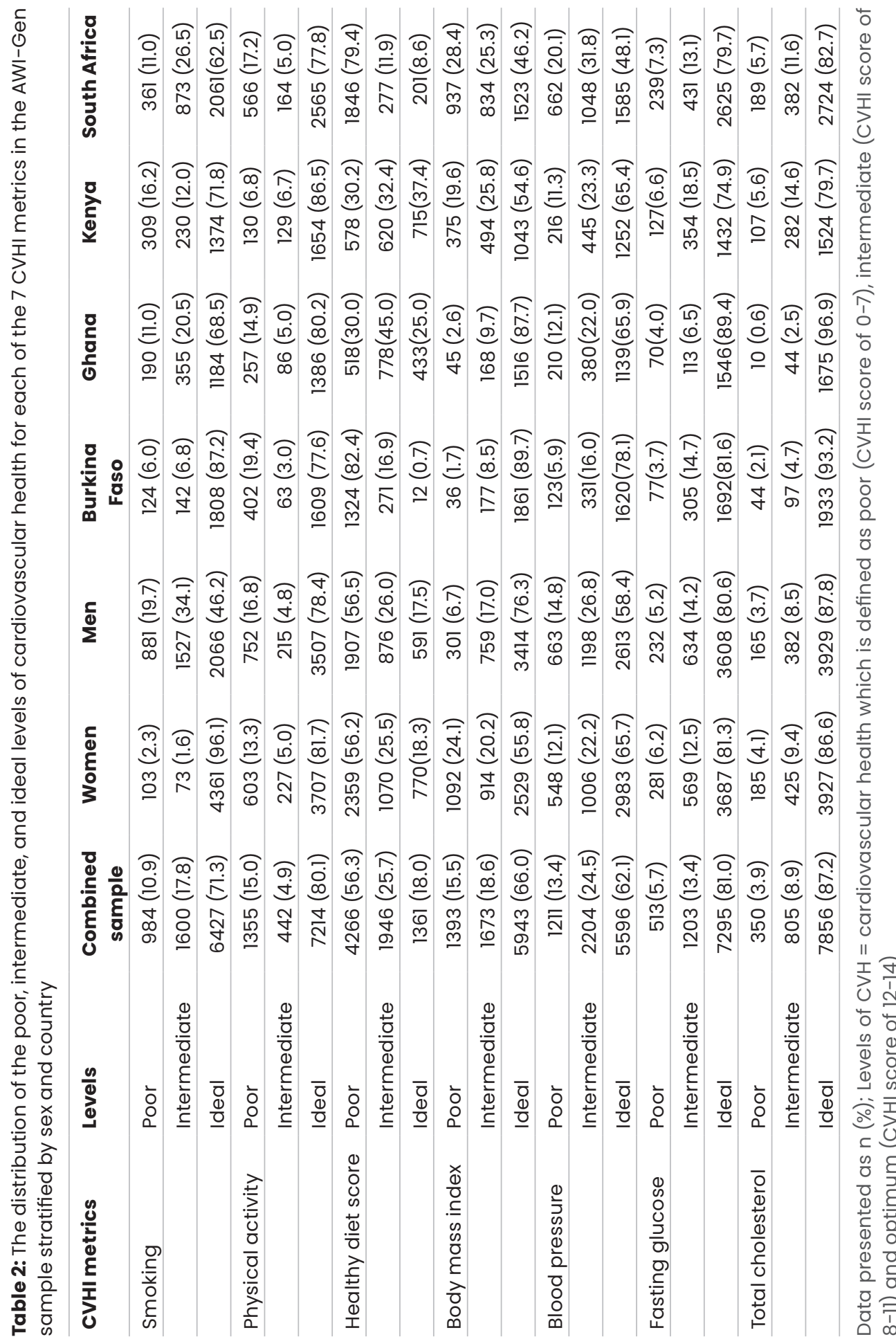


There were inverse linear associations between CIMT and CVHI in our combined population ( $r=-0.064 ; p<0.001)$ and for Burkina Faso $(r=-0.126 ; p<0.001)$, Ghana $(r=-0.085 ; p<0.001)$, Kenya $(r=-0.178 ; p<0.001)$ and South Africa $(r=-0.199$; $p<0.001$ ) and these are presented as line plots in Additional file 2: Figs S3 and S4. In the adjusted multilevel mixed-effects generalized linear regression model for the combined population a unit increase in the CVHI was associated with -5.54 $[-6.75,-4.32] \mu m$ decrease in common CIMT (Figure 2). The risk of atherosclerosis (high common CIMT) was progressively lower with increasing $\mathrm{CVHI}$ in each country, sex and household SES category in both the combined sample (Figure 2) and the sex stratified analyses (Figures 3 and 4). A similar inverse association was observed in women $(-4.44[-6.23,-2.65])$ and in men $(-6.27[-7.98,-4.64])$ (Figures 3 and 4, respectively).

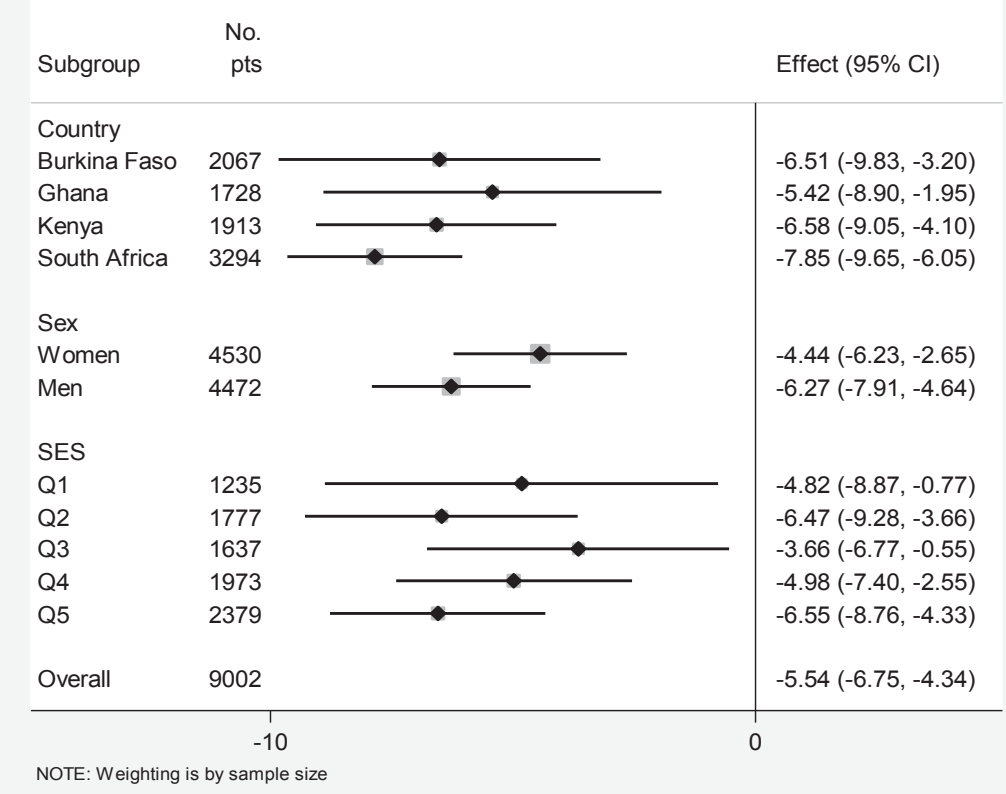

Figure 2: Adjusted* association of cardiovascular health index (CVHI) score with common carotid intima-media thickness (CIMT in $\mu \mathrm{m}$ ) in the combined AWI-Gen population stratified by country, sex and socio-economic status (SES) subgroups. Effect is represented by $\beta$-coefficients and is a change in mean CIMT (in $\mu \mathrm{m}$ ) with each unit increase in the CVHI score (0-14). Wealth quintile defined as Q1 = poorest, Q2 = poorer, Q3 = poor, Q4 less poor and Q5 = least poor. *Adjusted for age, educational status, household SES, HIV+ART, self-reported history of stroke, heart attack, congestive heart failure and chronic kidney disease. 


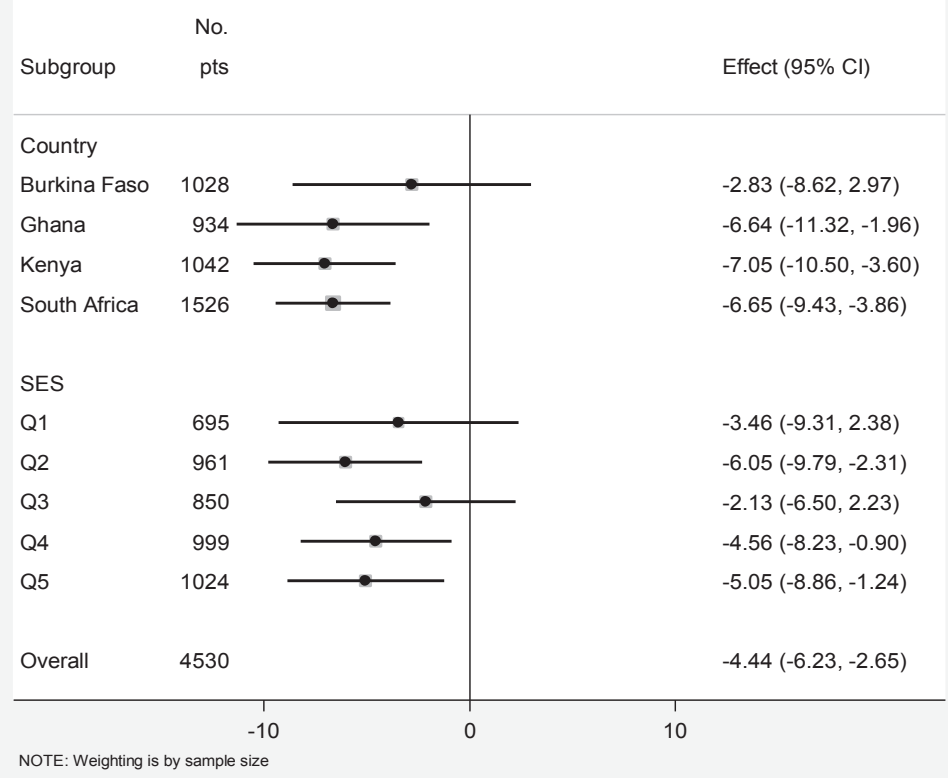

Figure 3: Adjusted* association of cardiovascular health index (CVHI) score with common carotid intima-media thickness (CIMT in $\mu \mathrm{m}$ ) in women stratified by country and socio-economic status (SES). Effect is represented by $\beta$-coefficients and is a change in mean CIMT (in $\mu \mathrm{m}$ ) with each unit increase in the CVHI score (0-14). Wealth quintile defined as Q1 = poorest, Q2 = poorer, Q3 = poor, Q4 less poor and Q5 = least poor. *Adjusted for age, educational status, household SES, HIV+ART, self-reported history of stroke, heart attack, congestive heart failure and chronic kidney disease. 


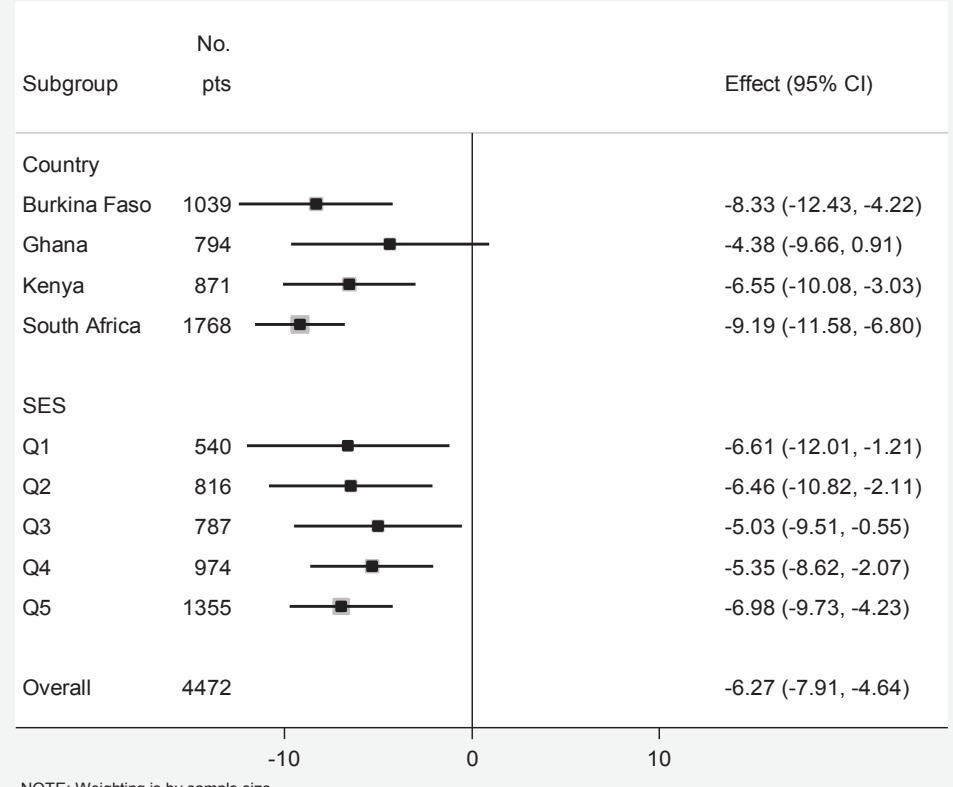

Figure 4: Adjusted* association of cardiovascular health index (CVHI) score with common carotid intima-media thickness (CIMT in $\mu \mathrm{m}$ ) in men stratified by country and socio-economic status (SES). Effect is represented by $\beta$-coefficients and is a change in mean CIMT (in $\mu \mathrm{m}$ ) with each unit increase in the CVHI score (0-14). Wealth quintile defined as Q1 = poorest, Q2 = poorer, Q3 = poor, Q4 less poor and Q5 = least poor. *Adjusted for age, educational status, household SES, HIV+ART, self-reported history of stroke, heart attack, congestive heart failure and chronic kidney disease.

Sequential adjustments of the regression models are presented in Table 3 and demonstrate minimal effects of adjustment on the $\beta$-coefficients. All models demonstrated a significant inverse association between the $\mathrm{CVHI}$ and common CIMT, except in women from Burkina Faso and men from Ghana, where the associations were again negative but with the upper $95 \% \mathrm{Cl}$ just overlapping zero. In adjusted analyses looking at the contribution of the individual components of $\mathrm{CVHI}$ to common CIMT, we observed that blood pressure, BMI and hyperglycemia were associated with common CIMT in the combined population, whilst smoking and physical activity were associated with common CIMT in women only (see Table 4). 
Table 3: Association of CVHI score with common CIMT $(\mu \mathrm{m})$ in women and men in the combined AWI-Gen sample and by countries

\begin{tabular}{|c|c|c|c|c|c|}
\hline & All countries & Burkina Faso & Ghana & Kenya & South Africa \\
\hline $\begin{array}{l}\text { Combined } \\
\text { sample }\end{array}$ & $\beta[95 \% \mathrm{Cl}]$ & $\beta[95 \% \mathrm{Cl}]$ & $\beta[95 \% \mathrm{Cl}]$ & $\beta[95 \% \mathrm{Cl}]$ & $\beta[95 \% \mathrm{Cl}]$ \\
\hline Model 1 & $\begin{array}{c}-7.39 \\
{[-8.49,-6.27]}\end{array}$ & {$[-9.80,-3.16]$} & $\begin{array}{c}-5.42 \\
{[-8.89,-1.94]}\end{array}$ & $\begin{array}{c}-6.79 \\
{[-9.24,-4.35]}\end{array}$ & $\begin{array}{c}-8.31 \\
{[-10.1,-6.52]}\end{array}$ \\
\hline Model 2 & $\begin{array}{c}-7.15 \\
{[-7.89,-6.41]}\end{array}$ & $\begin{array}{c}-6.43 \\
{[-9.76,-3.09]}\end{array}$ & $\begin{array}{c}-5.46 \\
{[-8.94,-1.97]}\end{array}$ & $\begin{array}{c}-6.66 \\
{[-9.12,-4.19]}\end{array}$ & $\begin{array}{c}-7.66 \\
{[-9.47,-5.85]}\end{array}$ \\
\hline Mod & $\begin{array}{c}-7.22 \\
{[-8.04,-6.39]}\end{array}$ & $\begin{array}{c}-6.51 \\
{[-9.83,-3.19]}\end{array}$ & $\begin{array}{c}-5.42 \\
{[-8.91,-1.94]}\end{array}$ & $\begin{array}{c}-6.57 \\
{[-9.05,-4.10]}\end{array}$ & $\begin{array}{c}-7.85 \\
{[-9.65,-6.05]}\end{array}$ \\
\hline Model 4 & $\begin{array}{c}-7.11 \\
{[-7.74,-6.48]}\end{array}$ & $\begin{array}{c}-6.42 \\
{[-9.75,-3.09]}\end{array}$ & $\begin{array}{c}-5.51 \\
{[-8.99,-2.02]}\end{array}$ & $\begin{array}{c}-6.63 \\
{[-9.10,-4.16]}\end{array}$ & $\begin{array}{c}-7.49 \\
{[-9.31,-5.68]}\end{array}$ \\
\hline \multicolumn{6}{|l|}{ Women } \\
\hline Model 1 & $\begin{array}{c}-5.98 \\
{[-7.44,-4.52]}\end{array}$ & $\begin{array}{r}-2.0 \\
{[-7.73,3}\end{array}$ & $\begin{array}{c}-6.04 \\
{[-10.60,-1.46]}\end{array}$ & $\begin{array}{c}-6.81 \\
{[-10.2,-3.43]}\end{array}$ & $\begin{array}{c}-6.89 \\
{[-9.69,-4.10]}\end{array}$ \\
\hline Mod & $\begin{array}{c}-5.85 \\
{[-7.00,-4.71]}\end{array}$ & $\begin{array}{c}-2.18 \\
{[-7.98,3.62]}\end{array}$ & $\begin{array}{c}-6.59 \\
{[-11.20,-1.96]}\end{array}$ & $\begin{array}{c}-7.05 \\
{[-10.50,-3.64]}\end{array}$ & $\begin{array}{c}-6.62 \\
{[-9.39,-3.86]}\end{array}$ \\
\hline Model 3 & $\begin{array}{c}-4.87 \\
{[-5.69,-4.05]}\end{array}$ & $\begin{array}{c}-2.83 \\
{[-8.63,2.97]}\end{array}$ & $\begin{array}{c}-6.64 \\
{[-11.30,-1.96]}\end{array}$ & $\begin{array}{c}-7.05 \\
{[-10.50,-3.59]}\end{array}$ & $\begin{array}{c}-6.65 \\
{[-9.44,-3.86]}\end{array}$ \\
\hline Model 4 & $\begin{array}{c}-5.93 \\
{[-7.02,-4.85]}\end{array}$ & $\begin{array}{c}-2.29 \\
{[-8.10,3.53]}\end{array}$ & $\begin{array}{c}-6.79 \\
{[-11.40,-2.15]}\end{array}$ & $\begin{array}{c}-7.16 \\
{[-10.60,-3.71]}\end{array}$ & $\begin{array}{c}-6.52 \\
{[-9.34,-3.69]}\end{array}$ \\
\hline \multicolumn{6}{|l|}{ Men } \\
\hline Model 1 & $\begin{array}{c}-7.83 \\
{[-9.79,-5.86]}\end{array}$ & $\begin{array}{c}-8.62 \\
{[-12.7,-4.52]}\end{array}$ & $\begin{array}{c}-4.69 \\
{[-10.1,0.66]}\end{array}$ & $\begin{array}{c}-6.66 \\
{[-10.20,-3.15]}\end{array}$ & $\begin{array}{c}-9.36 \\
{[-11.70,-7.01]}\end{array}$ \\
\hline Moc & $\begin{array}{c}-7.42 \\
{[-9.21,-5.63]}\end{array}$ & $\begin{array}{c}-8.25 \\
{[-12.4,-4.14]}\end{array}$ & $\begin{array}{c}-4.56 \\
{[-9.91,0.79]}\end{array}$ & $\begin{array}{c}-6.51 \\
{[-10.00,-2.99]}\end{array}$ & $\begin{array}{c}-8.84 \\
{[-11.20,-6.44]}\end{array}$ \\
\hline Model 3 & $\begin{array}{c}-5.68 \\
{[-6.57,-4.80]}\end{array}$ & $\begin{array}{c}-8.33 \\
{[-12.4,-4.22]}\end{array}$ & $\begin{array}{c}-4.38 \\
{[-9.67,0.92]}\end{array}$ & $\begin{array}{c}-6.55 \\
{[-10.10,-3.02]}\end{array}$ & $\begin{array}{c}-9.19 \\
{[-11.60,-6.80]}\end{array}$ \\
\hline Model 4 & $\begin{array}{c}-7.38 \\
{[-9.11,-5.66]}\end{array}$ & $\begin{array}{c}-8.18 \\
{[-12.3,-4.07]}\end{array}$ & $\begin{array}{c}-4.47 \\
{[-9.81,0.87]}\end{array}$ & $\begin{array}{c}-6.42 \\
{[-9.93,-2.91]}\end{array}$ & $\begin{array}{c}-8.71 \\
{[-11.10,-6.32]}\end{array}$ \\
\hline
\end{tabular}

Effect measured as $\beta$-coefficient (change in mean CIMT in $\mu \mathrm{m}$ per unit change in CVHI score); Model 1 adjusted for age and sex; Model 2 adjusted for age, sex, education status, household SES and site; Model 3 adjusted for self-reported history of stroke, heart attack, congestive heart failure and chronic kidney disease in addition to model 2; Model 4 was adjusted for model 2 plus chronic kidney disease and HIV plus antiretroviral therapy (ART) use; Model 1 for women and men were adjusted for only age. $\mathrm{CVHI}=$ cardiovascular health index; CIMT = carotid intima-media thickness. 


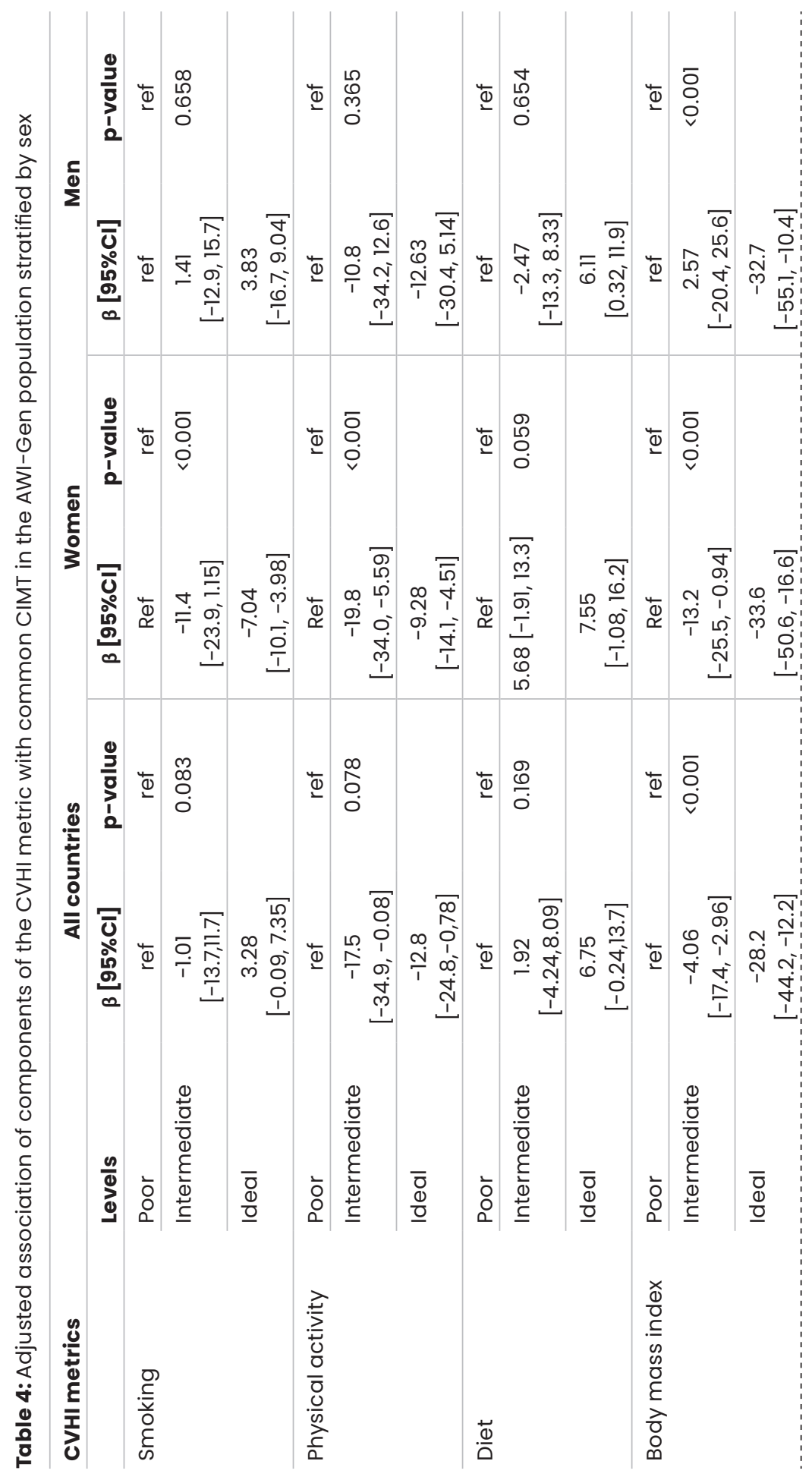




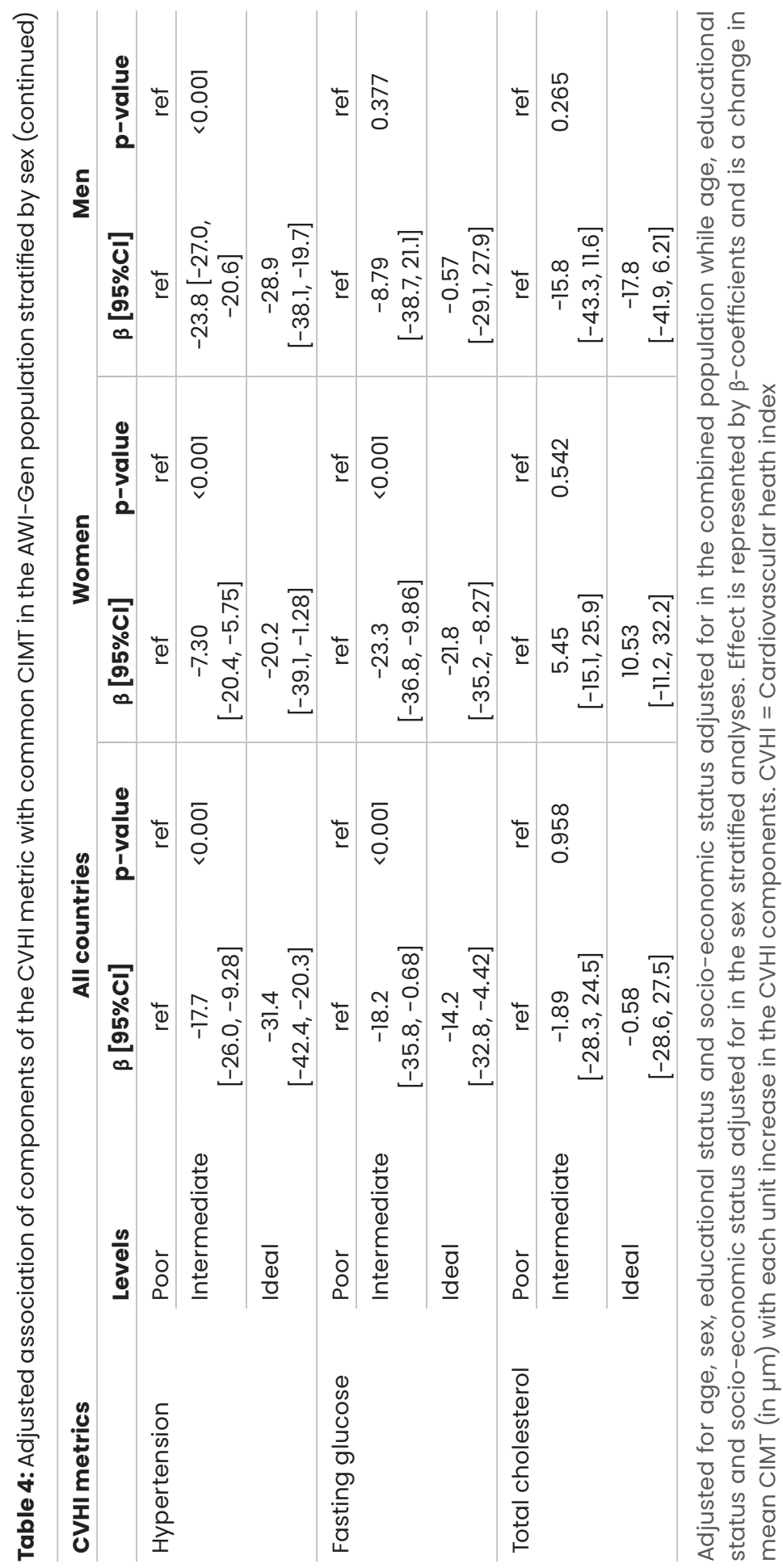




\section{Discussion}

Results from this large population-based study of middle-aged men and women from four countries in sub-Saharan Africa highlight relatively high levels of ideal cardiovascular health. We have also shown a significant inverse linear association between increasing $\mathrm{CVH}$ score and common CIMT independent of age, educational status, household socio-economic status, HIV infection and ART use and prior history of cardiovascular or chronic kidney diseases. To our knowledge this study is the largest to demonstrate an association between the CVHI metric and subclinical atherosclerosis in an apparently healthy adult population from multiple countries in SSA.

The prevalence of ideal cardiovascular health (iCVH) was 31.9\% in the combined AWI-Gen population and this was higher than the $6.8 \%$ reported in the multiethnic study of atherosclerosis (MESA) conducted among Caucasians, Chinese, African Americans and Hispanics in the United States (25). Similarly, data from the Behavioral Risk Factor Surveillance System study, which used information obtained telephonically from nearly 1.4 million subjects in the United States reported a much lower prevalence of iCVH (5.1\%) in 2009 than reported in the current study (28). The iCVH levels among our participants from Ghana was $50.9 \%$ and higher than reported by the Research on Obesity and Diabetes among African Migrants (RODAM) study conducted among Ghanaians residing in rural $(25.7 \%)$ and urban (7.5\%) areas and their counterparts in the diaspora $(<5 \%)(29)$. Further to this a systematic review by Peng et al, 2018 showed that participants from Africa were likely to have higher proportions of ideal CVH compared to North and South Americans, Asians, Europeans and the Oceania's (30). The RODAM study, however, used the comprehensive five dietary components (as described in the original CVHI) to define healthy diet whereas only two components were available for use in the AWI-Gen study. This may therefore result in over classifying AWI-Gen participants into the ideal diet category. This notwithstanding, the broad range of iCVH levels observed in the various settings may be a reflection of the level of ongoing epidemiological transition from communicable to non-communicable diseases. Thus, the iCVH levels were much higher in rural communities in Ghana and Burkina Faso and lower in the more urban settings of Kenya and South Africa and lowest in Europe and the USA.

We observed an inverse association of the iCVH with common CIMT levels independent of age, educational status, socio-economic status, HIV infection and ART use. Similar to our findings, several other studies conducted in Caucasian, African-American and Asian populations have observed increasing $\mathrm{CVHI}$ score is associated with lower risk of subclinical atherosclerosis $(15,17,31-$ 
35). It has been reported that a proportion of residual phenotypic variance is due to the additive effects of genes (36). Although, we did not adjust for the effect of genes in this paper, the Emory Twin Study did report that shared genetic and other familial factors do not confound the association of CVHI with CIMT (15). Our results did not show any significant disparities in the association between CVHI and CIMT across different levels of socioeconomic wealth quintiles as has been previously reported in a multi-ethnic population, including African Americans, in the United States (25). This may be due to the fact that participants in our population did not show significant difference in wealth distribution.

The rising burden of CVD morbidity and mortality in SSA has been reported to occur in younger age groups $(1,37)$. This, coupled with high levels of unawareness of risk factors and challenges with secondary prevention, calls for the evaluation of simple tools that can be used in routine screening for subjects with poor $\mathrm{CVH}$ in primary prevention efforts. The significant independent association of CIMT with the CVHI demonstrated in this study makes a strong case for the utility of the CVHI metric in the routine screening of CVD risk in the broader SSA population irrespective of SES status.

We observed in the combined sample that $\mathrm{BMI}$, blood pressure and hyperglycaemia were the CVHI metrics most strongly associated with common CIMT. The former 2 components were also associated with common CIMT in both women and men whilst physical activity and smoking related with common CIMT in women but not men. This could be due to the fact that women were less likely to smoke compared to men and equally had healthier physical activity levels than men. These findings have implications for prioritizing particular $\mathrm{CVHI}$ components for the primary prevention of atherosclerotic cardiovascular diseases in SSA. Although total cholesterol did not correlate with $\mathrm{CVHI}$, cholesterol levels in Africans remain lower than in the Western World. However with urbanisation cholesterol levels are increasing and thus may well contribute in the near future, to atherosclerotic cardiovascular disease in SSA (38).

Our study does have some limitations. Reliance on self-reported smoking, physical activity, HIV infection status and ART use may result in recall and social desirability biases. Diet was ascertained as self-reported servings of fruits and vegetables per day instead of derived from five components (fruits and vegetables per day; portions of fish a week; servings of fibre-rich whole grains per day; sodium per day and sugar-sweetened beverages per week) as recommended by AHA, which could have resulted in misclassification of certain participants in terms of their dietary intake. In addition, the cut points used for classifying each of the components of the $\mathrm{CVHI}$ metric were largely based 
on those used in populations of European ancestry. This brings into question whether these cut points are appropriate for use in African populations. However, the cut points used in the $\mathrm{CVHI}$ for defining cholesterol, blood pressure, glucose and BMI levels are used globally for the categorisation of these disease markers, and are recognised cut points in the clinical guidelines of many African nations; their use within these countries has not been widely questioned. Other cut points have been challenged for their use within African populations and include those for HDL and triglycerides (39), HbAlc (40) and waist circumference (41), but none of these factors are used within the $\mathrm{CVHI}$ metric.

Another possible shortcoming of the use of the CVHI in African populations is the high prevalence of underweight which is classified in the ideal category for BMI but is associated with increased cardiovascular risk. However, a large metaanalysis has shown that low $\mathrm{BMI}$ is more strongly associated with respiratory disease than CVD mortality, but it must be noted that this review included few studies from Africa (42). Future studies confirming the applicability of the CVHI criteria to African populations, especially those for BMI, would be important. In the current study it was essential to use the existing CVHI criteria so that we were able to compare our data to those from studies conducted in both African and non-African populations. Despite these limitations, the greatest strength of this work is that it used a large cohort of African individuals from 4 different SSA countries and a standardized data collection protocol, with laboratory assays performed at a single laboratory. In addition, a large number of possible confounding or modulatory variables were measured for use in the multiple regression models. Furthermore, this is the largest African study to use the CVHI to assess cardiovascular health and its association with common CIMT and provides important information on the utility of this metric for screening for individuals at high risk for CVD in resource-poor environments.

\section{Conclusion}

The CVHI score has a significant inverse, independent association with common CIMT in older adults across four sub-Saharan African countries. This makes a case for its use as a simple tool to monitor cardiovascular health at a population and clinical level in SSA. The current study demonstrates that primary prevention of atherosclerotic CVDs in SSA should focus on reducing smoking and obesity, improved control of hypertension and hyperglycemia and higher levels of physical activity. 


\title{
Supplementary Information
}

The online version contains supplementary material available at https://doi. org/10.1186/s12916-021-01909-6.

Additional file 1: Table s1. Scoring and definition of components of Cardiovascular Health Index

Additional file 2: Figure S1. Kernel density of mean distribution number of ideal cardiovascular health metrics (0-14) by AWI-Gen study countries. Figure $\mathbf{S 2}$. Kernel density of mean CIMT distribution in $\mu \mathrm{m}$ by AWI-Gen study countries. Figure S3. The linear association between CVH score and CIMT in the combined AWI-Gen population. Figure S4. The linear association between CVH score and CIMT in the combined four participating countries of the AWI-Gen study.

\begin{abstract}
Abbreviations
AHA/ASA: American Heart Association and the American Stroke Association; AWI-Gen: African Wits-INDEPTH Genomic studies; BMI: Body mass index; CVD: Cardiovascular disease; CIMT: Carotid-Intima media thickness; CVHI: Cardiovascular Health Index; HDSS: Health and Socio-demographic Surveillance System; SSA: Sub-Saharan Africa
\end{abstract}

\section{Acknowledgements}

We are grateful to the participants for their generosity and sacrifice for spending many hours responding to questionnaires, being measured, and having samples taken. We wish to acknowledge the sterling contributions of our field workers, phlebotomists, laboratory scientists, administrators, data personnel, and other staff who contributed to the data and sample collections, processing, storage, and shipping.

\section{Authors' contributions}

EAN, NJC, MLB, and KKG conceived the study and designed the analysis plan. EAN conducted the literature search, analyzed the data, and wrote the first draft. MLB, NJC, and KKG contributed to revising the text and writing the paper. EAN, NJC, ARO, GA, LKM, SFM, SSRC, PRB, HS, SMT, SAN, DEG, FJR, MLB, MR, and KKG critically reviewed the paper. $M R$ is overall principal investigator; $A R O, S M T$, and SAN are site principal investigators of the AWI-Gen study and together with sites investigators EAN, NJC, ARO, GA, LKM, SFM, PRB, HS, SMT, and SAN acquired data and had data oversight. All authors reviewed and approved the manuscript before submitting for publication. 


\section{Funding}

The AWI-Gen Collaborative Centre is funded by the National Human Genome Research Institute (NHGRI), Office of the Director (OD), Eunice Kennedy Shriver National Institute Of Child Health \& Human Development (NICHD), the National Institute of Environmental Health Sciences (NIEHS), the Office of AIDS Research (OAR), and the National Institute of Diabetes and Digestive and Kidney Diseases (NIDDK), of the National Institutes of Health under award number U54HG006938 and its supplements, as part of the H3Africa Consortium. Additional funding was leveraged from the Department of Science and Technology, South Africa, award number DST/CON 0056/2014. EAN receives support from the Global Health Support Program of the University Medical Center Utrecht (UMCU), Utrecht University, the Netherlands, and the Navrongo Health Research Centre (NHRC), Ghana.

\section{Availability of data and materials}

The datasets generated and/or analyzed during the current study will be made publicly available in the European Genome-phenome Archive under the set of projects related to the Human Heredity and Health in Africa (H3Africa) Consortium. Details concerning access to data and DNA can be found in the document titled H3Africa Data and Biospecimen Access Committee Guidelines, available in the consortium documents section of the H3Africa website (www. h3africa.com).

\section{Ethics approval and consent to participate}

This study received ethical approval from the Human Research Ethics Committee (Medical) of the University of the Witwatersrand (Wits) (protocol number M121029) and was renewed in 2017 (protocol number M170880). Additional approvals were obtained from the local ethics review boards in research site prior to commencing any participant-related activities.

\section{Consent for publication}

Not applicable

\section{Competing interests}

The authors declare that they have no competing interests. 


\section{References}

1. GBD 2015 Risk Factors Collaborators. Global, regional, and national comparative risk assessment of 79 behavioural, environmental and occupational, and metabolic risks or clusters of risks, 1990-2015: a systematic analysis for the Global Burden of Disease Study 2015. Lancet. 2016;388(10053):1659-724.

2. Roth GA, Johnson C, Abajobir A, Abd-Allah F, Abera SF, Abyu G, et al. Global, Regional, and National Burden of Cardiovascular Diseases for 10 Causes, 1990 to 2015. J Am Coll Cardiol. 2017;70(1):1-25.

3. Santosa A, Byass P. Diverse Empirical Evidence on Epidemiological Transition in Lowand Middle-Income Countries: Population-Based Findings from INDEPTH Network Data. PLoS One. 2016;11(5):e0155753.

4. Bawah A, Houle B, Alam N, Razzaque A, Streatfield PK, Debpuur C, et al. The Evolving Demographic and Health Transition in Four Low- and Middle-Income Countries: Evidence from Four Sites in the INDEPTH Network of Longitudinal Health and Demographic Surveillance Systems. PLoS One. 2016;11(6):e0157281.

5. D'Agostino RB, Sr., Vasan RS, Pencina MJ, Wolf PA, Cobain M, Massaro JM, et al. General cardiovascular risk profile for use in primary care: the Framingham Heart Study. Circulation. 2008;117(6):743-53.

6. Goff DC Jr, Lloyd-Jones DM, Bennett G, Coady S, D'Agostino RB Sr, Gibbons R, et al. 2013 ACC/AHA guideline on the assessment of cardiovascular risk: a report of the American College of Cardiology/American Heart Association Task Force on Practice Guidelines. J Am Coll Cardiol. 2014;1(63 (25 Pt B)):2935-59.

7. Boateng D, Agyemang C, Beune E, Meeks K, Smeeth L, Schulze MB, et al. Cardiovascular disease risk prediction in sub-Saharan African populations - Comparative analysis of risk algorithms in the RODAM study. Int J Cardiol. 2018;254:310-5.

8. Lloyd-Jones DM, Hong Y, Labarthe D, Mozaffarian D, Appel LJ, Van Horn L, et al. Defining and setting national goals for cardiovascular health promotion and disease reduction: the American Heart Association's strategic Impact Goal through 2020 and beyond. Circulation. 2010;121(4):586-613.

9. Shay CM, Gooding HS, Murillo R, Foraker R. Understanding and Improving Cardiovascular Health: An Update on the American Heart Association's Concept of Cardiovascular Health. Prog Cardiovasc Dis. 2015;58(1):41-9.

10. Shay CM, Ning H, Daniels SR, Rooks CR, Gidding SS, Lloyd-Jones DM. Status of cardiovascular health in US adolescents: prevalence estimates from the National Health and Nutrition Examination Surveys (NHANES) 2005-2010. Circulation. 2013;127(13):1369-76.

11. Huffman MD, Capewell S, Ning H, Shay CM, Ford ES, Lloyd-Jones DM. Cardiovascular health behavior and health factor changes (1988-2008) and projections to 2020: results from the National Health and Nutrition Examination Surveys. Circulation. 2012;125(21):2595-602. 
12. Touboul PJ, Grobbee DE, den Ruijter H. Assessment of subclinical atherosclerosis by carotid intima media thickness: technical issues. Eur J Prev Cardiol. 2012;19(2 Suppl):18-24.

13. Bots ML, Evans GW, Tegeler CH, Meijer R. Carotid Intima-media Thickness Measurements: Relations with Atherosclerosis, Risk of Cardiovascular Disease and Application in Randomized Controlled Trials. Chin Med J 2016;129(2):215-26.

14. Nonterah EA, Boua PR, Klipstein-Grobusch K, Asiki G, Micklesfield LK, Agongo G, et al. Classical Cardiovascular Risk Factors and HIV are Associated With Carotid IntimaMedia Thickness in Adults From Sub-Saharan Africa: Findings From H3Africa AWI-Gen Study. J Am Heart Assoc. 2019;8(14).

15. Kulshreshtha A, Goyal A, Veledar E, McClellan W, Judd S, Eufinger SC, et al. Association between ideal cardiovascular health and carotid intima-media thickness: a twin study. J Am Heart Assoc. 2014;3(1):e000282.

16. Saleem Y, DeFina LF, Radford NB, Willis BL, Barlow CE, Gibbons LW, et al. Association of a favorable cardiovascular health profile with the presence of coronary artery calcification. Circ Cardiovasc Imaging. 2015;8(1).

17. Younus A, Aneni EC, Spatz ES, Osondu CU, Roberson L, Ogunmoroti O, et al. A Systematic Review of the Prevalence and Outcomes of Ideal Cardiovascular Health in US and Non-US Populations. Mayo Clin Proc. 2016;91(5):649-70.

18. Feinstein MJ, Kim JH, Bibangambah P, Sentongo R, Martin JN, Tsai AC, et al. Ideal Cardiovascular Health and Carotid Atherosclerosis in a Mixed Cohort of HIV-Infected and Uninfected Ugandans. AIDS Res Hum Retroviruses. 2017;33(1):49-56.

19. Ketelaar EJ, Vos AG, Godijk NG, Scheuermaier K, Devillé W, Tempelman H, et al. Ideal Cardiovascular Health Index and Its Determinants in a Rural South African Population. Global Heart. 2020;15(1).

20. Ramsay M, Crowther NJ, Tambo E, Agongo G, Baloyi V, Dikotope S, et al. H3Africa AWIGen Collaborative Centre: a resource to study the interplay between genomic and environmental risk factors for cardiometabolic diseases in four sub-Saharan African countries. Global Health, Epidemiology and Genomics. 2016;1(e20):1-13.

21. Ali SA, Soo C, Agongo G, Alberts M, Amenga-Etego L, Boua RP, et al. Genomic and environmental risk factors for cardiometabolic diseases in Africa: methods used for Phase 1 of the AWI-Gen population cross-sectional study. Glob Health Action. 2018;11(sup2):1507133.

22. WHO. Global Recommendations on Physical Activity for Health. Geneva, Switzerland: World Health Organisation; 2010.

23. Ferro-Luzzi A, Garza C, Haas J, Habicht JP, Himes J, Pradilla A, et al. Physical Status: The use and interpretation of anthropometry. Geneva, Switzerland: World Health Organisation; 1995.

24. Klug E, Raal FJ, Marais AD, Taskinen M-R, Dalby AJ, Schamroth $C$, et al. South African dyslipidaemia guideline consensus statement. S Afr Med J. 2012;102(3 Pt 2):178-87.

25. Polonsky TS, Ning H, Daviglus ML, Liu K, Burke GL, Cushman M, et al. Association of Cardiovascular Health With Subclinical Disease and Incident Events: The Multi-Ethnic Study of Atherosclerosis. J Am Heart Assoc. 2017;6(3). 
26. Shea S, Lima J, Diez-Roux A, Jorgensen NW, McClelland RL. Socioeconomic Status and Poor Health Outcome at 10 Years of Follow-Up in the Multi-Ethnic Study of Atherosclerosis. PLoS One. 2016;11(11):e0165651.

27. Rundek T, Brown DL. Socioeconomic status and subclinical atherosclerosis: are we closing disparity gaps? Stroke. 2014;45(4):948-9.

28. Pilkerton CS, Singh SS, Bias TK, Frisbee SJ. Changes in Cardiovascular Health in the United States, 2003-2011. J Am Heart Assoc. 2015;4(9):e001650.

29. van Nieuwenhuizen B, Zafarmand MH, Beune E, Meeks K, Aikins AD, Addo J, et al. Ideal cardiovascular health among Ghanaian populations in three European countries and rural and urban Ghana: the RODAM study. Intern Emerg Med. 2018;13(6):845-56.

30. Peng Y, Cao S, Yao Z, Wang Z. Prevalence of the cardiovascular health status in adults: A systematic review and meta-analysis. Nutr Metab Cardiovasc Dis. 2018;28(12):1197207.

31. Xanthakis V, Enserro DM, Murabito JM, Polak JF, Wollert KC, Januzzi JL, et al. Ideal cardiovascular health: associations with biomarkers and subclinical disease and impact on incidence of cardiovascular disease in the Framingham Offspring Study. Circulation. 2014;130(19):1676-83.

32. Shpilsky D, Bambs C, Kip K, Patel S, Aiyer A, Olafiranye O, et al. Association between ideal cardiovascular health and markers of subclinical cardiovascular disease. Clin Cardiol. 2018;41(12):1593-9.

33. Singh SS, Pilkerton CS, Shrader CD, Jr., Frisbee SJ. Subclinical atherosclerosis, cardiovascular health, and disease risk: is there a case for the Cardiovascular Health Index in the primary prevention population? BMC Public Health. 2018;18(1):429.

34. Santos IS, Goulart AC, Pereira AC, Lotufo PA, Bensenor IM. Association between Cardiovascular Health Score and Carotid Intima-Media Thickness: Cross-Sectional Analysis of the Brazilian Longitudinal Study of Adult Health (ELSA-Brasil) Baseline Assessment. J Am Soc Echocardiogr. 2016;29(12):1207-16 e4.

35. Gao J, Bao M, Liu Y, Shi J, Huang Z, Xing A, et al. Changes in cardiovascular health score and atherosclerosis progression in middle-aged and older persons in China: a cohort study. BMJ Open. 2015;5(8):e007547.

36. Kathiresan S, Srivastava D. Genetics of human cardiovascular disease. Cell. 2012;148(6):1242-57.

37. Yuyun MF, Sliwa K, Kengne AP, Mocumbi AO, Bukhman G. Cardiovascular Diseases in Sub-Saharan Africa Compared to High-Income Countries: An Epidemiological Perspective. Global Heart. 2020;15(1).

38. Noubiap JJ, Bigna JJ, Nansseu JR, Nyaga UF, Balti EV, Echouffo-Tcheugui JB, et al. Prevalence of dyslipidaemia among adults in Africa: a systematic review and metaanalysis. The Lancet Global Health. 2018;6(9):e998-e1007.

39. Bentley AR, Rotimi CN. Interethnic Differences in Serum Lipids and Implications for Cardiometabolic Disease Risk in African Ancestry Populations. Glob Heart. 2017;12(2):141-50. 
40. Zemlin AE, Matsha TE, Hassan MS, Erasmus RT. HbAlc of $6.5 \%$ to diagnose diabetes mellitus -- does it work for us? -- the Bellville South Africa study. PLoS One. 2011;6(8):e22558.

41. Ekoru K, Murphy GAV, Young EH, Delisle H, Jerome CS, Assah F, et al. Deriving an optimal threshold of waist circumference for detecting cardiometabolic risk in sub-Saharan Africa. Int J Obes 2017:1-8.

42. Di Angelantonio E, Bhupathiraju SN, Wormser D, Gao P, Kaptoge S, de Gonzalez AB, et al. Body-mass index and all-cause mortality: individual-participant-data metaanalysis of 239 prospective studies in four continents. The Lancet. 2016;388(10046):77686. 


\section{Additional file 1}

Table S1: Scoring and definition of components of Cardiovascular Health Index

\begin{tabular}{|c|c|c|c|}
\hline CVHI metric & Levels & Score & Definition \\
\hline \multirow[t]{3}{*}{ Smoking* } & Poor & 0 & Current \\
\hline & Intermediate & 1 & Former \\
\hline & Ideal & 2 & Never \\
\hline \multirow{3}{*}{$\begin{array}{l}\text { Physical } \\
\text { activity }\end{array}$} & Poor & 0 & No physical activity \\
\hline & Intermediate & 1 & 1-149mins/week of MVPA ${ }^{\dagger}$ \\
\hline & Ideal & 2 & 2150mins/week of MVPA \\
\hline \multirow{3}{*}{$\begin{array}{l}\text { Healthy diet } \neq \\
\text { score }\end{array}$} & Poor & 0 & < 2 servings per day \\
\hline & Intermediate & 1 & 2 to 4 servings per day \\
\hline & Ideal & 2 & $\geq 5$ servings per day \\
\hline \multirow{3}{*}{$\begin{array}{l}\text { Body mass } \\
\text { index }\end{array}$} & Poor & 0 & $\geq 30 \mathrm{~kg} / \mathrm{m}^{2}$ \\
\hline & Intermediate & 1 & 25 to $29.99 \mathrm{~kg} / \mathrm{m}^{2}$ \\
\hline & Ideal & 2 & $<25 \mathrm{~kg} / \mathrm{m}^{2}$ \\
\hline \multirow{3}{*}{$\begin{array}{l}\text { Blood } \\
\text { pressure }\end{array}$} & Poor & 0 & $\mathrm{SBP} \geq 140$ or $\mathrm{DBP} \geq 90 \mathrm{~mm} \mathrm{Hg}$ \\
\hline & Intermediate & 1 & $\begin{array}{l}\text { SBP } 120-139 \text { or DBP } 80-89 \mathrm{~mm} \text { Hg or treated } \\
\text { with antihypertensive to normal level }\end{array}$ \\
\hline & Ideal & 2 & $\begin{array}{l}\mathrm{SBP}<120 \text { or } \mathrm{DBP}<80 \mathrm{~mm} \mathrm{Hg} \text {, without } \\
\text { antihypertensive medication }\end{array}$ \\
\hline \multirow{3}{*}{$\begin{array}{l}\text { Fasting } \\
\text { glucose }\end{array}$} & Poor & 0 & $\geq 126 \mathrm{mg} / \mathrm{dL}$ \\
\hline & Intermediate & 1 & $\begin{array}{l}100 \text { to } 125 \mathrm{mg} / \mathrm{dL} \text { or treated with anti-diabetes } \\
\text { to normal level }\end{array}$ \\
\hline & Ideal & 2 & $<100 \mathrm{mg} / \mathrm{dL}$, without anti-diabetes medication \\
\hline \multirow{3}{*}{$\begin{array}{l}\text { Total } \\
\text { cholesterol }\end{array}$} & Poor & 0 & $\geq 240 \mathrm{mg} / \mathrm{dL}$ \\
\hline & Intermediate & 1 & 200 to $239 \mathrm{mg} / \mathrm{dL}$ or treated to $<200 \mathrm{mg} / \mathrm{dL}$ \\
\hline & Ideal & 2 & $<200 \mathrm{mg} / \mathrm{dL}$, without lipid lowering medication \\
\hline
\end{tabular}

*Self-reported smoking status was reported as never smoked, previous smoker and current smoker; there was no data on duration of smoking; †MVPA indicates moderateto-vigorous physical activity; †Healthy diet score modified based on available data in the AWI-Gen study on the number of servings of fruits and vegetable per day. 


\section{Additional file 2}

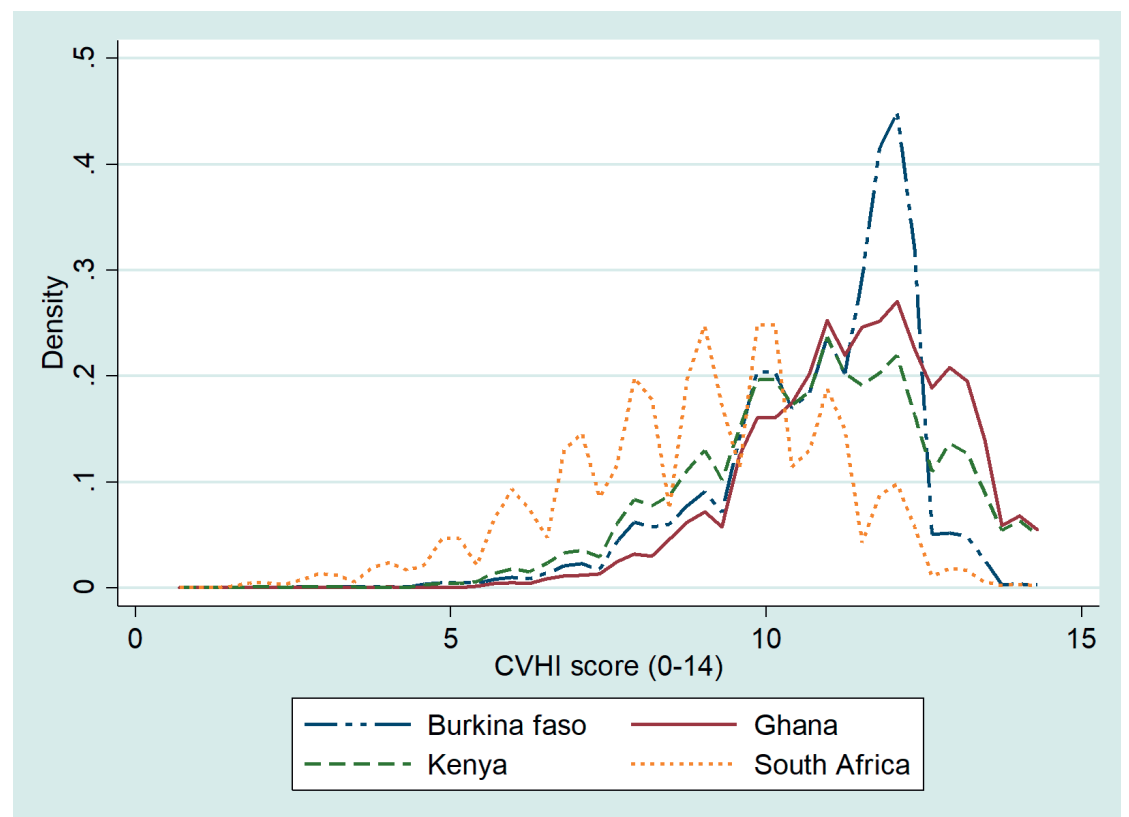

Figure S1: Kernel density of mean distribution number of ideal cardiovascular health metrics (0-14) by AWI-Gen study countries

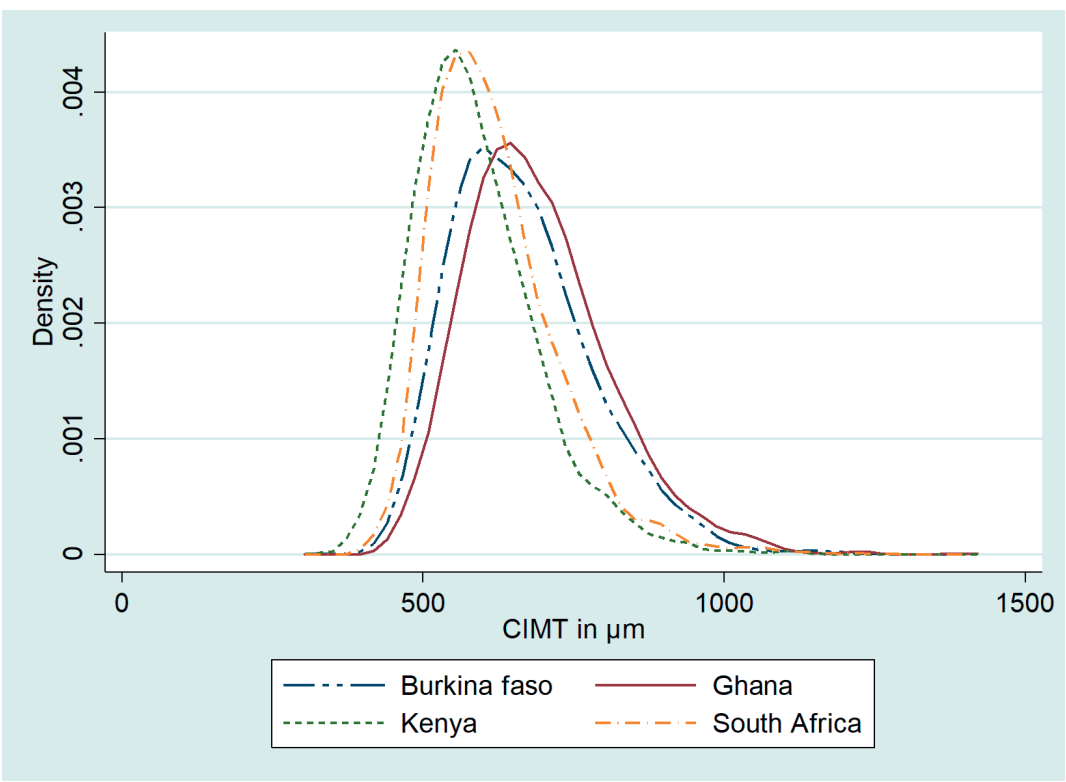

Figure S2: Kernel density of mean CIMT distribution in $\mu \mathrm{m}$ by AWI-Gen study countries 


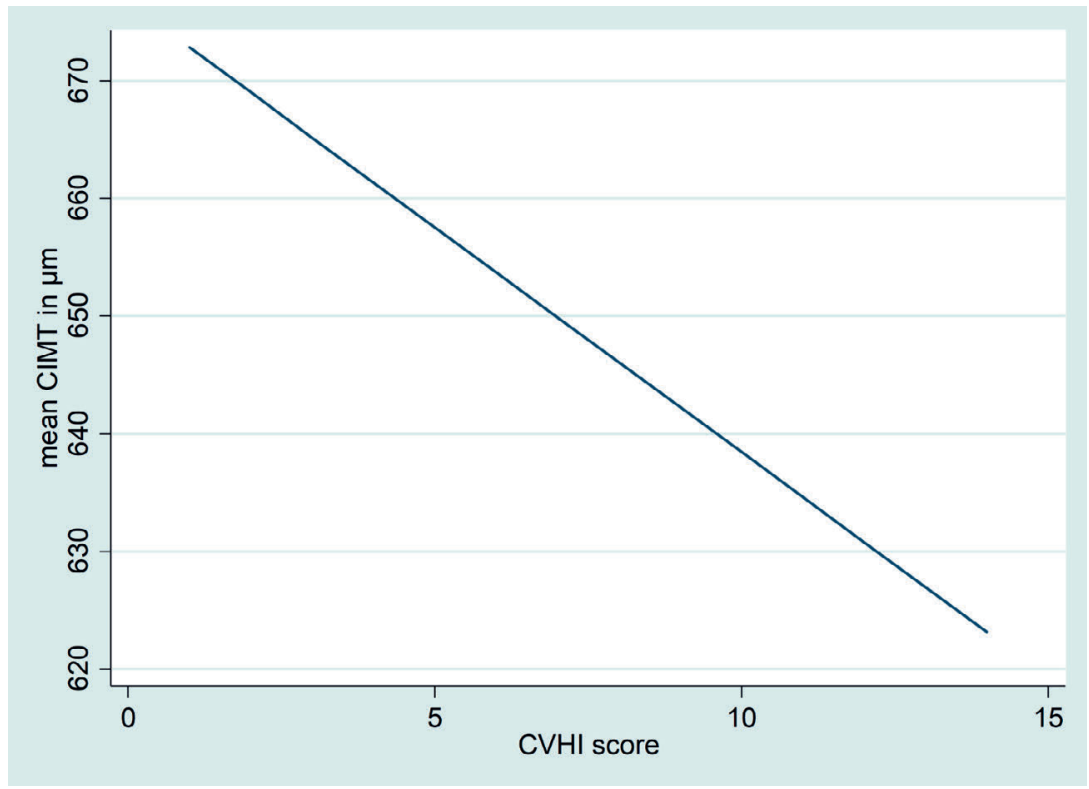

Figure S3: The linear association between CVH score and CIMT in the combined AWIGen population
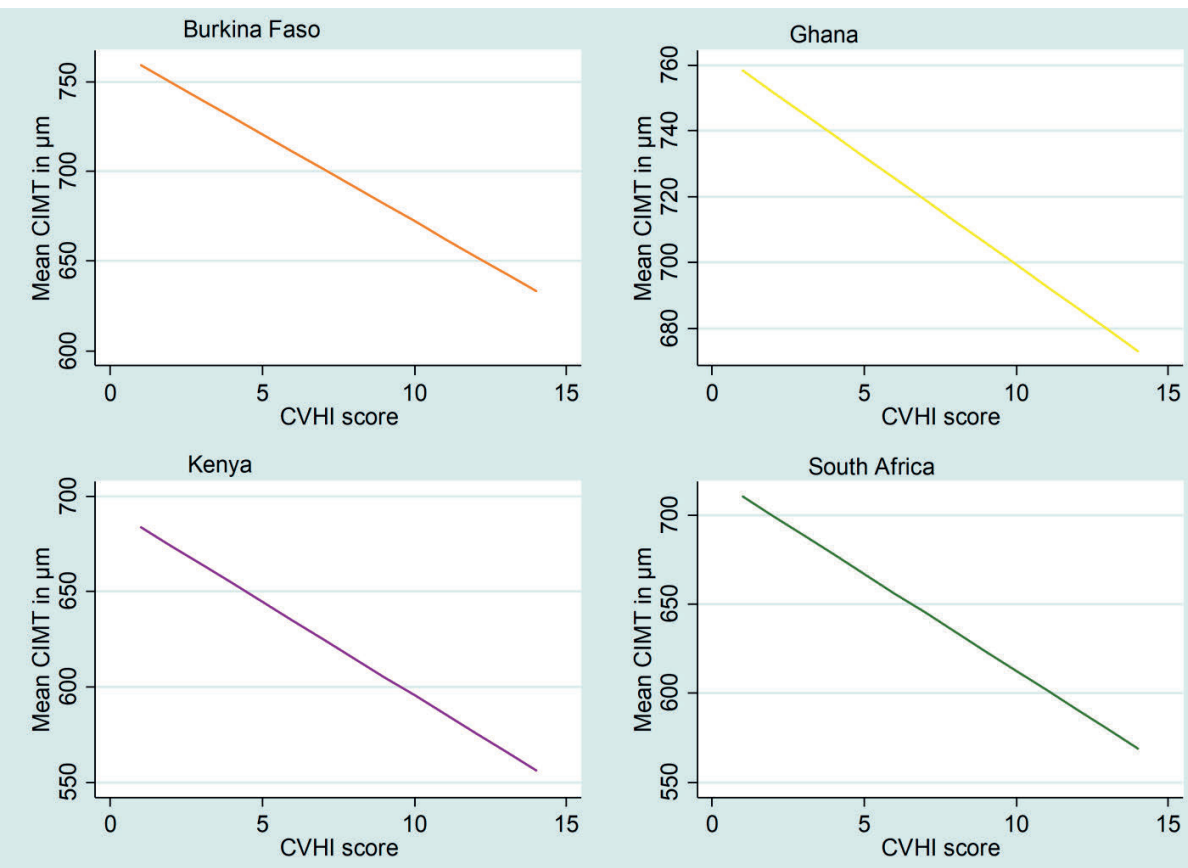

Figure S4: The linear association between CVH score and CIMT in the combined four participating countries of the AWI-Gen study 

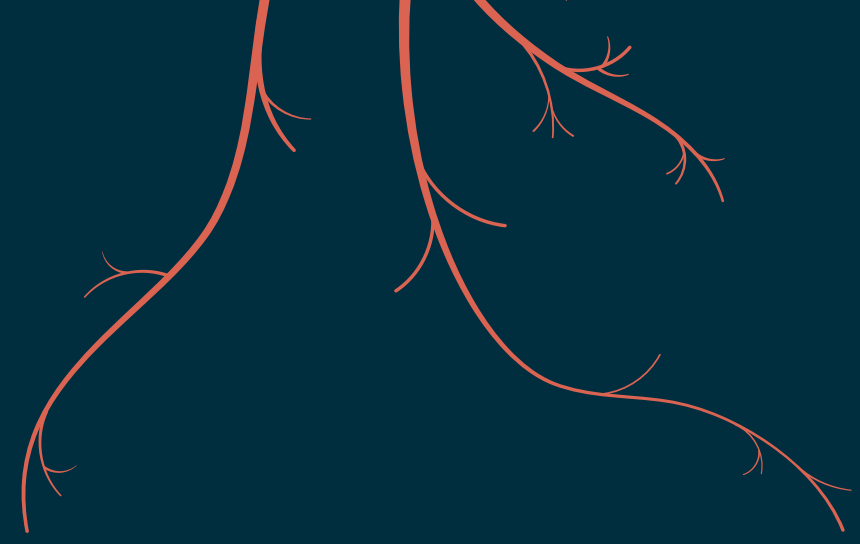

\title{
Chapter 6
}

\author{
Carotid atherosclerosis, \\ microalbuminuria and predicted \\ 10-year atherosclerotic cardiovascular \\ disease risk in sub-Saharan Africa: \\ an H3Africa AWI-Gen study
}

Nonterah EA, Boateng D, Crowther NJ, Klipstein-Grobusch K, Oduro AR, Agongo G, Mohamed SF, Boua PR, Choma SSR, Norris SA, Tollman SM, Bots ML, Ramsay M and Grobbee DE, for the H3Africa AWI-Gen study. 


\section{Abstract}

Importance: Carotid atherosclerosis and microalbuminuria predict atherosclerotic cardiovascular disease (ASCVD) but are understudied in subSaharan African (SSA).

Objective: We determined the association of these factors with 10-year ASCVD risk in middle-aged Africans.

Design, settings and participants: A cross sectional analyses of baseline data from the African-Wits-INDEPTH genomic study (AWI-Gen). Apparently healthy women and men aged 40-60 years drawn from surveillance sites from Burkina Faso, Ghana, Kenya and South Africa. Additional recruitment was from urban Soweto in Johannesburg, South Africa.

Main outcome and measures: Hypotheses for the analyses formulated after data collection. The main measures were classical cardiovascular risk factors, carotid atherosclerosis assessed using carotid intima-media thickness (CIMT) using B-mode ultrasound and microalbuminuria measured using spot urine albumin (SUA) and urine albumin-creatinine-ratio (UACR). The main outcome being high 10-year ASCVD risk defined as the 2018 Pooled Cohort Equations score 27.5. Associations were determined using multivariable logistic regression analysis.

Findings: We studied 9011 participants with an average age of $50 \pm 6$ years. High CIMT, SUA and UACR were each associated with older age, and high prevalence of both diabetes and hypertension (all associations $p<0.01$ ). Smokers were likely to have higher SUA and UACR. Carotid atherosclerosis was common in Burkina Faso and Ghana while microalbuminuria was common in Kenya and South African. The SUA was associated with higher odds of carotid atherosclerosis compared to UACR. Common CIMT, SUA and UACR were strongly associated with 10-year ASCVD risk. CIMT had a higher odds ratio (95\% confidence interval) for 10-year ASCVD risk in both women $(1.95[1.78,2.14])$ and men $(1.73[1.55,1.93])$ compared to SUA $(1.27[1.12,1.43]$ and $1.40[1.26,1.55]$, respectively) and UACR $(1.30$ $[1.10,1.54]$ and $1.30[1.15,1.46]$, respectively).

Conclusion and relevance: The presence of microalbuminuria measured by spot urine albumin may indicate risk of subclinical carotid atherosclerosis and high 10-year CVD risk in middle-aged individuals from SSA. Due to limited regional variation in our findings, SUA may be used to assess CVD risk in both sexes across many African populations. These data must be confirmed in longitudinal studies of cardiovascular events.

Keywords: Carotid atherosclerosis; Microalbuminuria; Pooled Cohort Equation; Atherosclerotic cardiovascular disease risk; sub-Saharan Africa 


\section{Introduction}

Cardiovascular disease (CVD) is a leading cause of death and disability worldwide (1) with low- and middle-income countries, including the subSaharan Africa (SSA) region, currently experiencing $80 \%$ of these CVD-related global deaths (2). Most of the CVD-related deaths in SSA occur in younger age groups when compared to the rest of the world (3) posing both health and economic concerns as these individuals represent the more economically productive sectors of society.

Established classical cardiovascular risk factors are major contributors to CVD in many populations. However, these factors alone do not explain the total risk for CVD and several novel factors such as markers of inflammation, atherosclerosis and microalbuminuria have been reported to contribute to CVD risk $(4,5)$. Common carotid intima-media thickness (CIMT) assessed by B-mode ultrasound is a marker of carotid atherosclerosis which often occurs early in life as a low grade sustained inflammatory process $(6,7)$ and progresses with age and is augmented by the presence of CVD risk factors. It is an established major precursor to the development of CVD later in life and has the ability to predict future cardiovascular events in the general population $(5,8)$.

Microalbuminuria has been reported to be an independent predictor of future cardiovascular events such as congestive heart failure, stroke and myocardial infarction $(9,10)$ especially in high risk groups such as subjects with hypertension or diabetes. However, few studies in high income countries have reported the association of microalbuminuria with CVD in the general population.

Despite being increasingly recognized as risk factors for cardiovascular events, there are few studies in African populations examining the association of microalbuminuria and carotid atherosclerosis with CVD. This is partly due to the difficulty in assessing cardiovascular risk in Africa as a result of the absence of data on clinical events. The PURE and INTERHEART studies are the only studies to have investigated the association of classical risk factors with CVD end points using African populations (11, 12). These studies were limited by their small sample sizes and in the case of the PURE study by selection bias, and neither study examined novel CVD risk factors. Thus, population level data on the association of CVD risk factors with clinical endpoints among individuals free of cardiovascular disease is still largely absent in SSA. This has led to the use of equations to assess the risk of CVD events in SSA populations. A commonly used prediction score is the 2018 revised Pooled Cohort Equation (PCE) which has a high level of accuracy for 10-year ASCVD risk prediction (13). The PCE is 
also known to perform very well in ethnic minority groups and included data from a large population of African American subjects (13).

It is important to conduct studies on the association of both classical and novel risk factors with CVD risk because the prevalence of these diseases is increasing across Africa (3) and therefore cost effective methods for assessing the presence of CVD are necessary. Thus, it is important to determine if an easily measured index such as microalbuminuria is associated with carotid atherosclerosis and whether it can be used as an indicator of CVD risk. Furthermore, comparing the ability of CIMT and microalbuminuria to predict CVD will offer new insights on factors that can be used to identify individuals at a high risk of CVD in population level screening programmes. This will also contribute baseline information as a benchmark for future research. Therefore, the aim of this study was to measure the association of microalbuminuria with CIMT and the association of both these variables with predicted 10-year ASCVD risk (using the PCE) in a large population of middle-aged adults drawn from Burkina Faso, Ghana, Kenya and South Africa. A secondary aim was to compare two commonly used methods for assessing microalbuminuria i.e. spot urine albumin (SUA) and urine albumin-creatinine ratio (UACR) for their association with CIMT and 10-year ASCVD risk.

\section{Methods}

\section{Study design and population}

We conducted a population-based cross-sectional study using the Human Hereditary and Health Africa (H3A) African-Wits-INDEPTH Genomic Study (AWIGen) cohort $(14,15)$. Data were collected from adults residing in health and socio-demographic surveillance sites (HDSS) located in Nanoro (Burkina Faso), Navrongo (Ghana), Nairobi (Kenya) and DIMAMO and Agincourt in South Africa (16). In addition, data was collected from the BT20+ (Birth to Twenty Plus) cohort study run by the Developmental Pathways to Health Research Unit (DPHRU) in Soweto, South Africa (16).

Study participants were adults aged 40-60 years residing in the various study sites and women included were not pregnant at the time of data collection. Details of the recruitment and data collection procedures for AWI-Gen have been published (16). Ineligible participants included those who self-reported a previous diagnosis of CVD (based on an affirmative response to the questions, "Has a doctor or other health professional ever told you that you had coronary heart disease, angina (also called angina pectoris), heart attack (also called myocardial infarction), or stroke?". Participants were also ineligible if their 
measured CIMT was greater than $1.5 \mathrm{~mm}$, as this was consistent with plaque and not atherosclerosis and so not relevant for this study (17).

\section{Data collection}

A structured questionnaire for obtaining information on socio-demographic variables, clinical history and behavioral risk factors was administered by trained researchers. Physical measurements included weight and height for computing body mass index (BMI), waist and hip circumference and blood pressure. Blood samples were used to assess fasting levels of glucose, lipids and insulin while urine samples were used to assess albumin and creatinine. Data collection tools were standardized across sites with centralized training of research coordinators and research officers to minimize variability in the measurements. Community engagement, broad consent as well as individual informed consent were obtained prior to carrying out study procedures. Details of data collection instruments and study procedure have been published elsewhere (16).

\section{Predicted 10-year atherosclerotic cardiovascular disease (ASCVD) risk}

Estimated 10-year ASCVD risk was computed using the revised 2018 PCE (13). This risk equation estimates the 10-year absolute risk of ASCVD, defined as death due to coronary heart disease or nonfatal myocardial infarction or fatal or nonfatal stroke over a 10-year period in people free of established CVD. The PCE permits the derivation of sex- and race-specific estimates of the 10-year risk for ASCVD for adults aged 40-79 years (which falls within the age category used by the AWI-Gen study of 40-60 years). Variables in the PCE include age, sex, black race, tobacco smoking, total and high-density lipoprotein cholesterol, treated or untreated systolic blood pressure and diabetes status. A PCE score $\leq 7.5 \%$ classifies an individual participant at a "high ASCVD risk" while a score above 7.5 is low risk (18).

Age and sex were self-reported at the time of data collection. Smoking was selfreported as cigarette use in the twelve months prior to data collection. Blood pressure was measured with the participant seated and with the arm resting on a chair at the level of the heart. An appropriate sized cuff was then applied and three readings taken with the first discarded while an average of the last two was used as the blood pressure reading. Fasting blood samples were taken for the measurement of fasting glucose and lipid fractions. These measurements were performed in a single laboratory using standardized procedures. The coefficient of variation of the glucose and lipid measurements ranged between $0.2 \%$ and $1.5 \%$. Diabetes status was defined as fasting glucose levels $27 \mathrm{mmol} / \mathrm{I}$ and/or known diabetic on medication at the time of data collection. 


\section{Carotid atherosclerosis}

\section{Common carotid intima-media thickness (CIMT)}

A LOGIQ e B-mode ultrasound system (GE Healthcare, Chicago, IL, USA) with a 12L-RS straight transducer was used to measure right and left intima-media thickness of the common carotid arteries (CCA). The IMT was measured within a $10 \mathrm{~mm}$ segment of the CCA starting from $1 \mathrm{~cm}$ away from the bulb. Measurements were taken of minimum, maximum and average distance in millimeters to two decimal places. Centralized training of selected staff (including nurses, clinicians and researchers) from study sites minimized measurement variability and reduced errors. Common CIMT $\geq 0.90 \mathrm{~mm}$ was used as the cut-point for diagnosing carotid atherosclerosis (19-22).

\section{Microalbuminuria}

\section{Spot urine albuminuria (SUA)}

Midstream urine samples (10-15 $\mathrm{ml}$ voided into a sterile collection pot) were collected in accordance with the procedures followed by the World Health Organization (WHO) (16). Samples were centrifuged at 1500-2000 $\mathrm{xg}$ for 5 minutes and the supernatant aliquoted into cryovials for storage at $-80^{\circ} \mathrm{C}$ until analysis. Albumin concentrations in urine samples were determined using a turbidimetric assay on a Cobas 501 autoanalyser (Roche Diagnostics, Manheim, Germany). The measuring range was $3-400 \mathrm{mg} / \mathrm{l}$ for albumin, and the coefficient of variation was less than $2.7 \%$. The use of spot urine as opposed to 24-hour urine collection or albumin-creatinine-ratio is previously validated in the general population $(23,24)$. Microalbuminuria was defined as spot urine albumin concentration $220 \mathrm{mg} / \mathrm{L}$ (or $20-400 \mathrm{mg} / \mathrm{L}$ within the confines of the measuring range of the study population) (25).

\section{Urine albumin-creatinine ratio (UACR):}

Urinary albumin concentration was measured with immunoturbidimetry as described above and urine creatinine was measured by an IDMS-traceable Jaffe method on the Cobas 501 instrument (Roche Diagnostics, Mannheim, Germany). The measuring range for the urine creatinine assay was 3.75- 550 $\mathrm{mmol} / \mathrm{I}$ with a coefficient of variation of $2.8 \%$. Urine ACR of more than $3 \mathrm{mg} /$ mmol was defined as albuminuria $(25,26)$.

\section{Statistical analyses}

Characteristics of the AWI-Gen participants are described using counts (n) and proportions (\%) for categorical data and means \pm standard deviations for the continuous data (all were normally distributed). The results were stratified by CIMT and microalbuminuria cut points. Differences between these groups were 
determined using Pearson's Chi squared test for categorical data and Student t-test for continuous data.

To determine the association between microalbuminuria and CIMT mixedeffect logistic regression analysis was performed using country as a random effect. This was done for the combined sample and for women and men. Adjustments were made for classical CVD risk factors (age, sex except in the sex stratified analyses, smoking, BMI, systolic blood pressure, total cholesterol, $\mathrm{HDL}$ ). Associations are reported as odds ratios with $95 \%$ confidence intervals (Cl) and were presented as forest plots.

The association of carotid atherosclerosis and microalbuminuria with 10year atherosclerotic cardiovascular disease (ASCVD) risk was determined using adjusted logistic regression analyses. Due to differences in their units of measurements, we first generated sex and country specific z-scores for common CIMT, spot urine and urine albumin-creatinine-ratio thus allowing direct comparison of the odds ratios. The z-scores were calculated using the following equation: $(x-\mu) / \sigma$ where $x$ is the raw, individual value, $\mu$ is the population mean, and $\sigma$ is the population standard deviation. As per the recommendations of the American Heart Association (AHA), a high 10-year ASCVD risk is defined as a PCE score $\mathbf{2 7 . 5 \%}$. We therefore generated a binary form of PCE and applied adjusted logistic regression analyses to determine the relationship of CIMT, SAU and UACR with high 10-year CVD risk. Adjustments were made in a sequential manner using the following variables sex (except in sex stratified analyses), educational level, household socio-economic status, body mass index (BMI), and participating country (except in the country specific analyses). The factors used for adjustment of the regression models were socio-demographic factors, which have been demonstrated to be associated with CVD, and BMI which is a well-recognized risk factor for CVD. The Akaike/Bayesian Information Criteria $(\mathrm{AIC} / \mathrm{BIC})$ were used to compare the models and the model with the lowest value was considered a better model. This was further confirmed using the HosmerLemeshow $(\mathrm{H}-\mathrm{L})$ goodness of fit test. Specifically the test determines whether the observed odds ratios (with $95 \% \mathrm{Cl}$ ) match the expected odds ratios in the population and population sub-groups (countries and sex specific subgroups) and a $p>0.05$ was considered a good fit.

For the country level comparisons of the strength of association of CIMT, SUA and UACR z-scores with high 10-year CVD risk across, we further conducted an inverse variance (IV) logistic regression meta-analysis. The best fitted model (fully adjusted model i.e. model 3) was used for these country and sex comparative analyses. The adjusted odds ratios are presented as forest plots with the within group variance measured using $I$-squared $\left(l^{2}\right)$ with a high 
score and $\mathrm{p}<0.05$ indicating high within group variance. Heterogeneity was measured to assess differences between study groups with a p>0.05 indicating low heterogeneity between groups. The statistical significance for all inferential statistics was set at a two-tailed $p<0.05$. All analyses were conducted using STATA v14.2 (StataCorp, College Station, TX, USA).

\section{Ethical clearance}

The AWI-Gen study received ethics approval from the Human Research Ethics Committee of the University of the Witwatersrand, Johannesburg, South Africa (ethics approval identification number: M121029, renewed in 2017 with number: M170880). Ethics approvals were also obtained from relevant ethics bodies in the participating sites and written informed consent obtained from all participants prior to recruitment.

\section{Data availability}

The AWI-Gen data used in the current study can be accessed from the European Genome-phenome Archive (www.ega459archive.org/datasets/ EGAD00001006425) with phenotype dataset accession ID of EGA00001002482.

\section{Results}

\section{Study population basic characteristics}

Presented in Table 1 is a comparison of the characteristics of the 9010 study participants according to carotid atherosclerosis and microalbuminuria status. Higher levels of education were associated with lower levels of atherosclerosis but higher levels of microalbuminuria while socio-economic status did not differ. Ghana and Burkina Faso had higher levels of carotid atherosclerosis than those from Kenya and South Africa. Kenya and South Africa had higher levels of microalbuminuria than Burkina Faso and Ghana. Participants with carotid atherosclerosis (high CIMT) and microalbuminuria (high SUA or high uACR) were more likely to be diabetic or hypertensive. The percentage of participants with high ASCVD risk (PCE 27.5) was significantly higher in the subject groups with high CIMT, SUA and UACR levels (Table 1). 


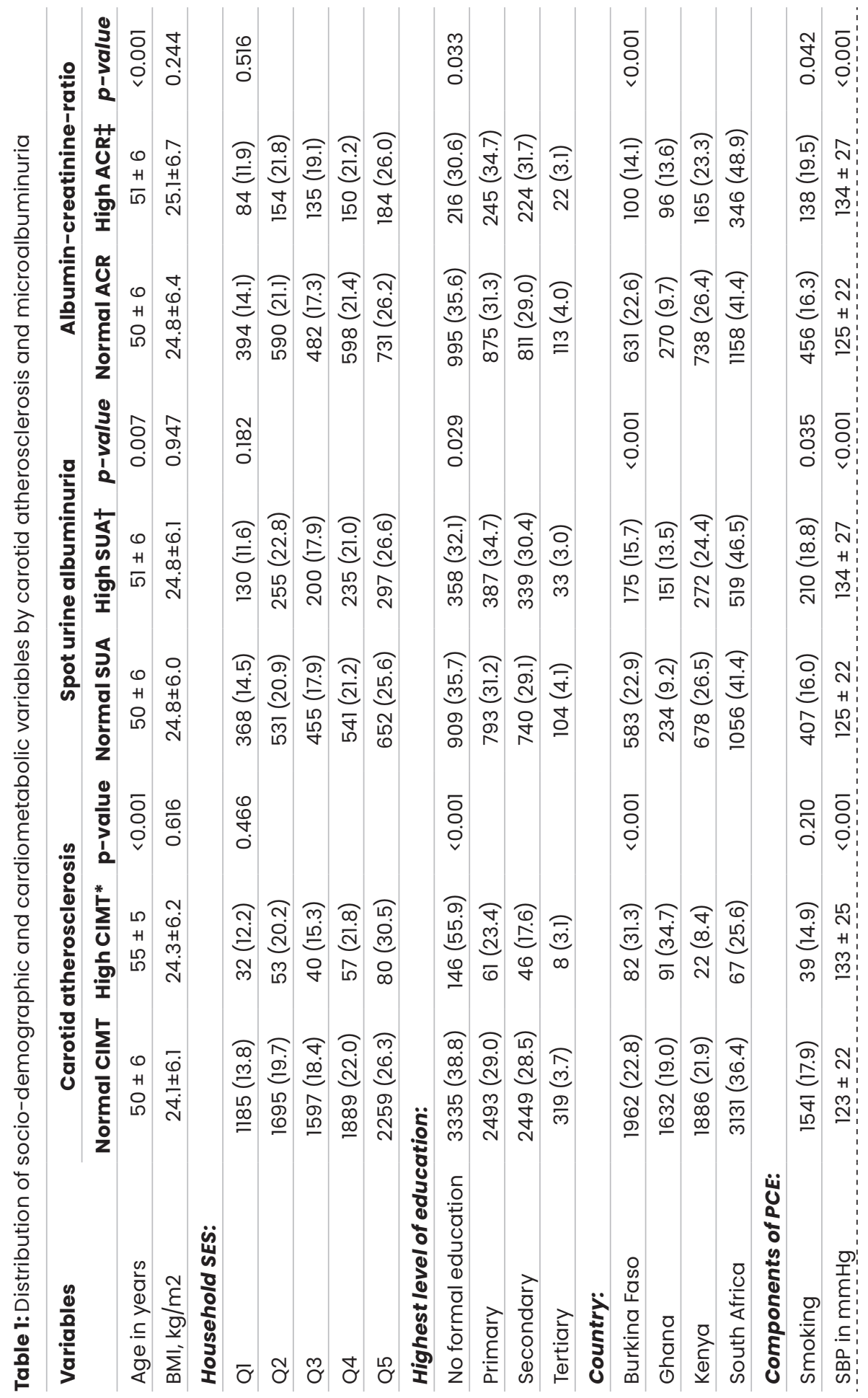




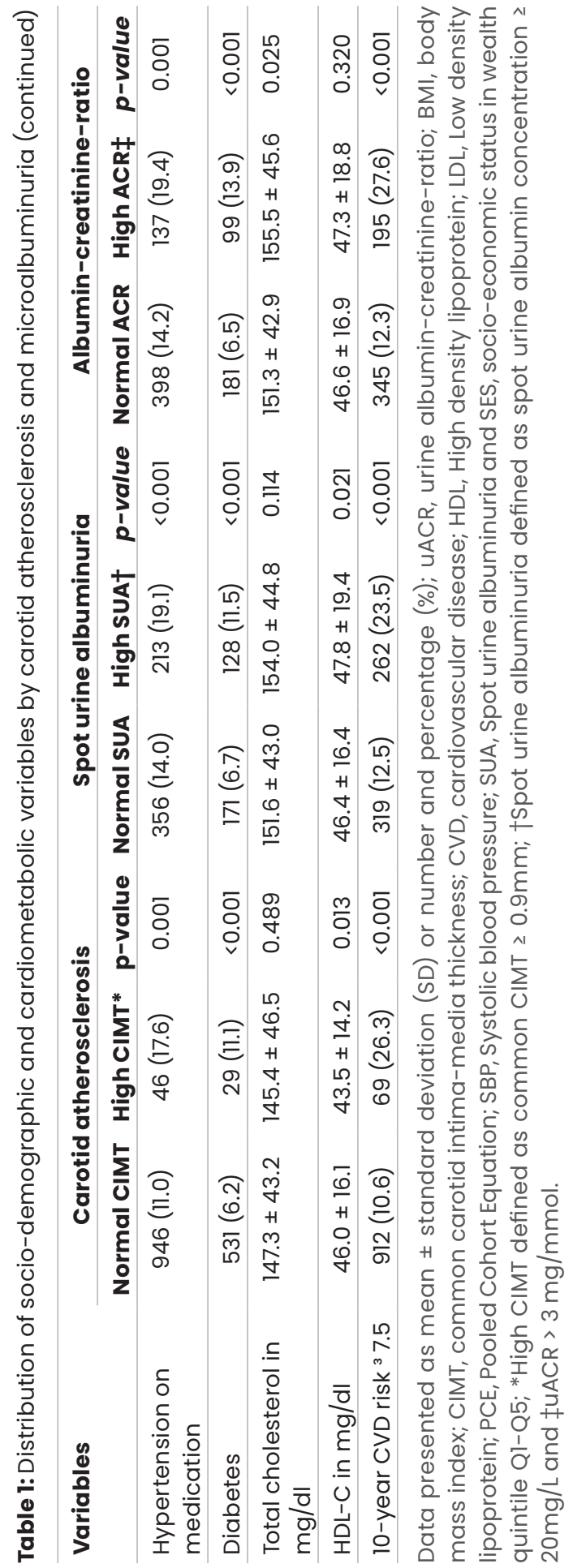




\section{Association of microalbuminuria with carotid intima-media thickness}

The associations of microalbuminuria with CIMT in the combined sample and in women and men are presented in Figure 1. In the combined population high SUA displayed a significant odds ratio for subclinical atherosclerosis (adjusted odds ratio $1.77,95 \%$ confidence interval $[1.04,3.01])$ in the combined population but this was not significant in women and men. The UACR was not associated with a higher risk for elevated CIMT in women and men or in the combined population (see Figure 1). There was significant within country variance in the measured SUA and UACR in the combined population and in men with minimal variance in women. There was no heterogeneity between women and men $(p=0.895)$. The country stratified analyses are presented in supplementary Figure Sl.

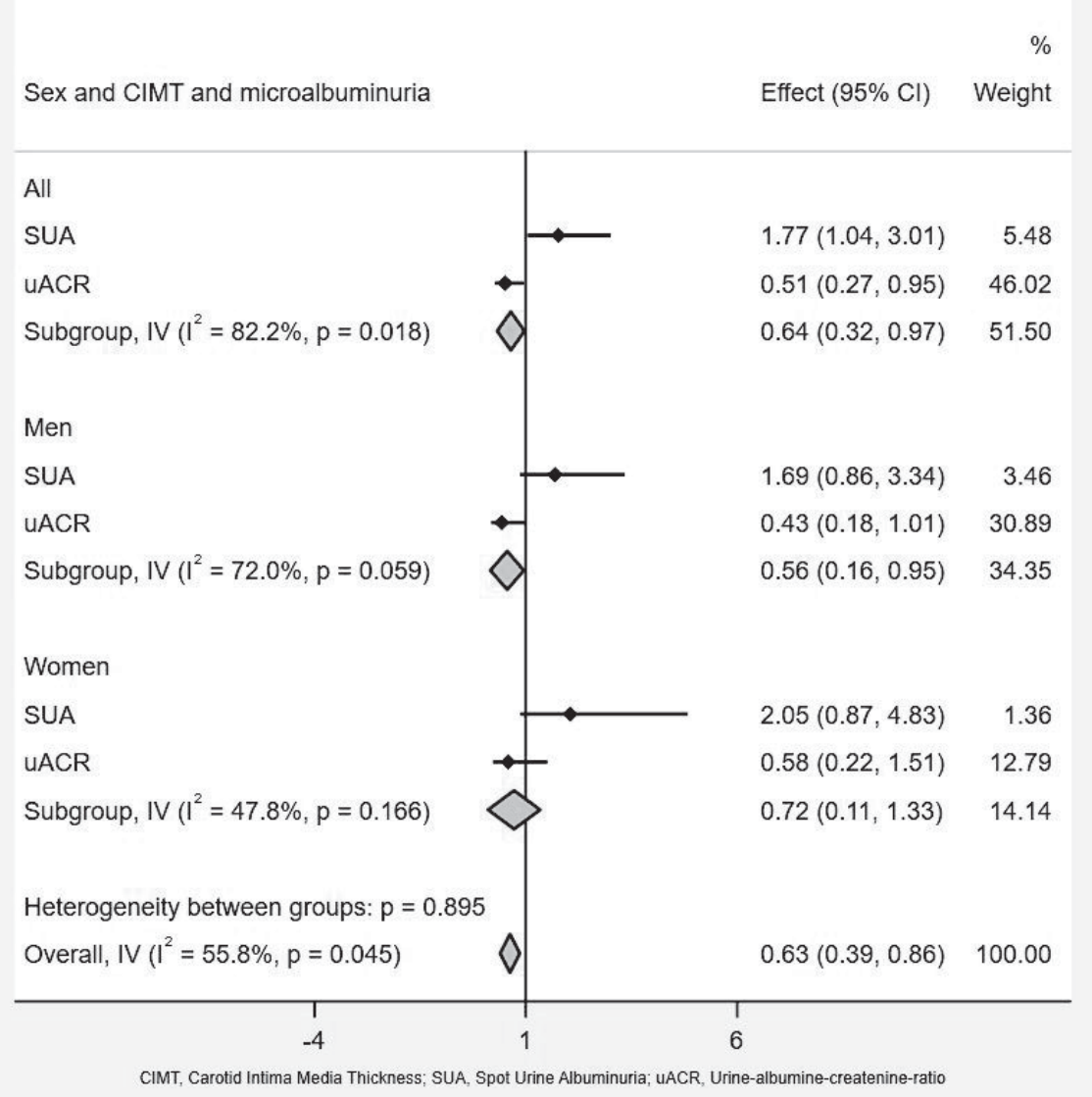

Figure 1: Association of microalbuminuria with CIMT in the combined study populations and in women and men; IV, inverse variance 


\section{Association of carotid atherosclerosis and microalbuminuria with high 10- year ASCVD risk}

Presented in Table 2 (combined population) is the association of carotid atherosclerosis or microalbuminuria with high 10-year ASCVD risk, stratified by sex. These associations were determined through sequential model building. In the combined population common CIMT and both measures of microalbuminuria were associated with 10-year ASCVD risk in all the models (for women and men). Thus, in model 3 for the combined population, common CIMT had higher odds of 10-year ASCVD risk (odds ratio 1.85, 95\% confidence interval $[1.75,1.99])$ compared to SUA $(1.34[1.24,1.45])$ and UACR $(1.30[1.18,1.43])$. This observed pattern was similar in women and men (see Table 2). The fully adjusted models (model 3 ) had lower AIC/BIC values indicating that they fitted the data better. This was further confirmed by the Hosmer-Lemeshow $(\mathrm{H}-\mathrm{L})$ test which showed $\mathrm{p}$-values greater than 0.05 for carotid atherosclerosis and microalbuminuria in the combined population and for women and men (Table 2). 


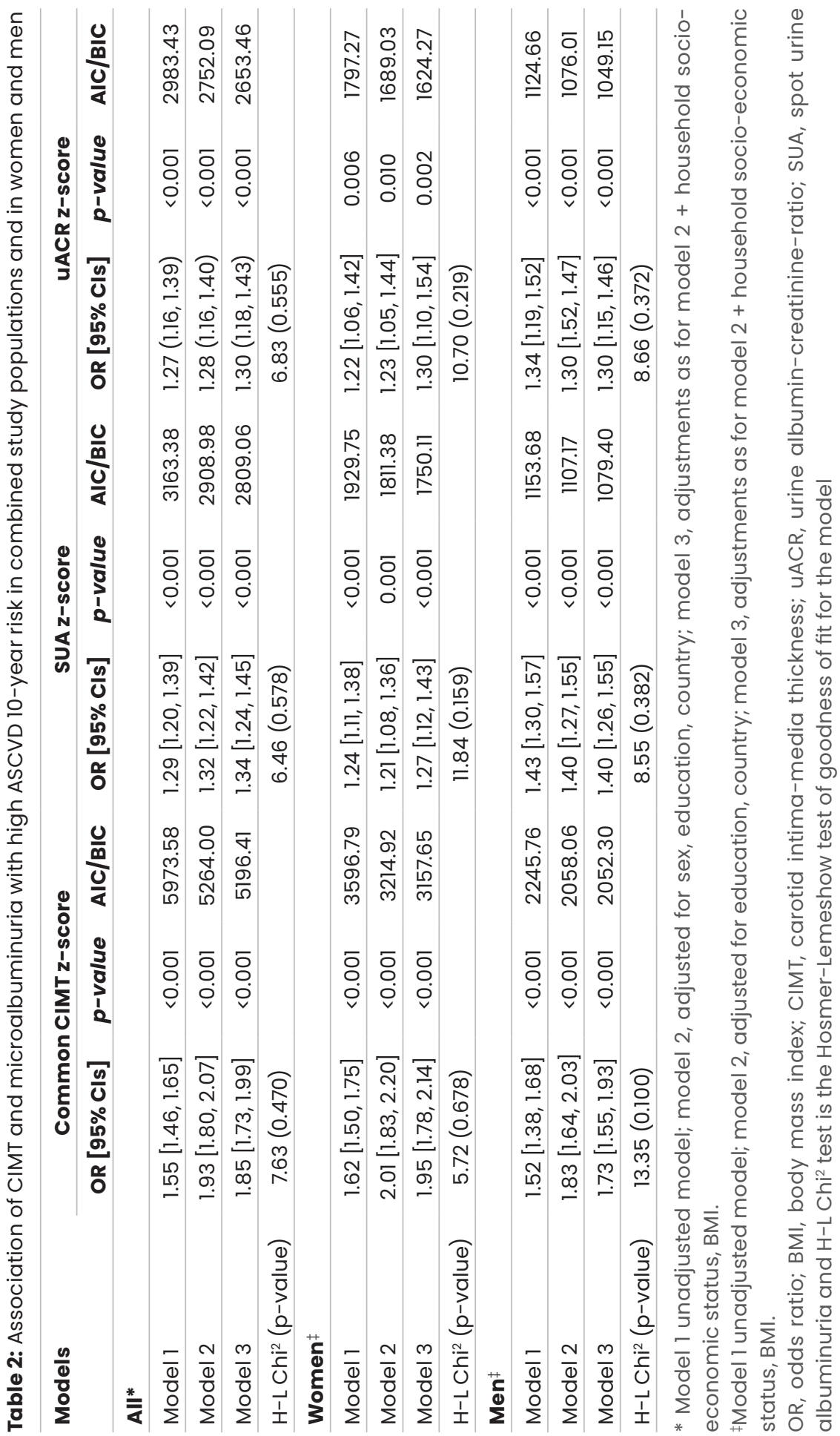


The fully adjusted model was thus used in meta-analyses to compare associations of carotid atherosclerosis and microalbuminuria with 10year ASCVD risk for the various countries stratified by sex (Figures 2 and 3). These analyses show that across all countries microalbuminuria and carotid atherosclerosis were significantly associated with 10-year ASCVD risk in women and men. However the magnitude of these associations differs between the countries and for women and men. In most instances, the strength of association of CIMT with ASCVD risk was higher than for SUA and UACR with few exceptions. Men from Ghana had higher odds of ASCVD risk from SUA than CIMT and UACR. In women from Kenya UACR presented with a higher odds of ASCVD risk than CIMT and SUA. The within country variance for women was higher for measured SUA $\left(I^{2}=74.6 \%, p=0.008\right)$ with the other measures not showing any significant variance across the countries. Among men there was no significant within country variance in the measures for carotid atherosclerosis and microalbuminuria. The heterogeneity between the z-scores for SUA, UACR and CIMT was significant in women $(p<0.001)$ and men $(p=0.002)$. 


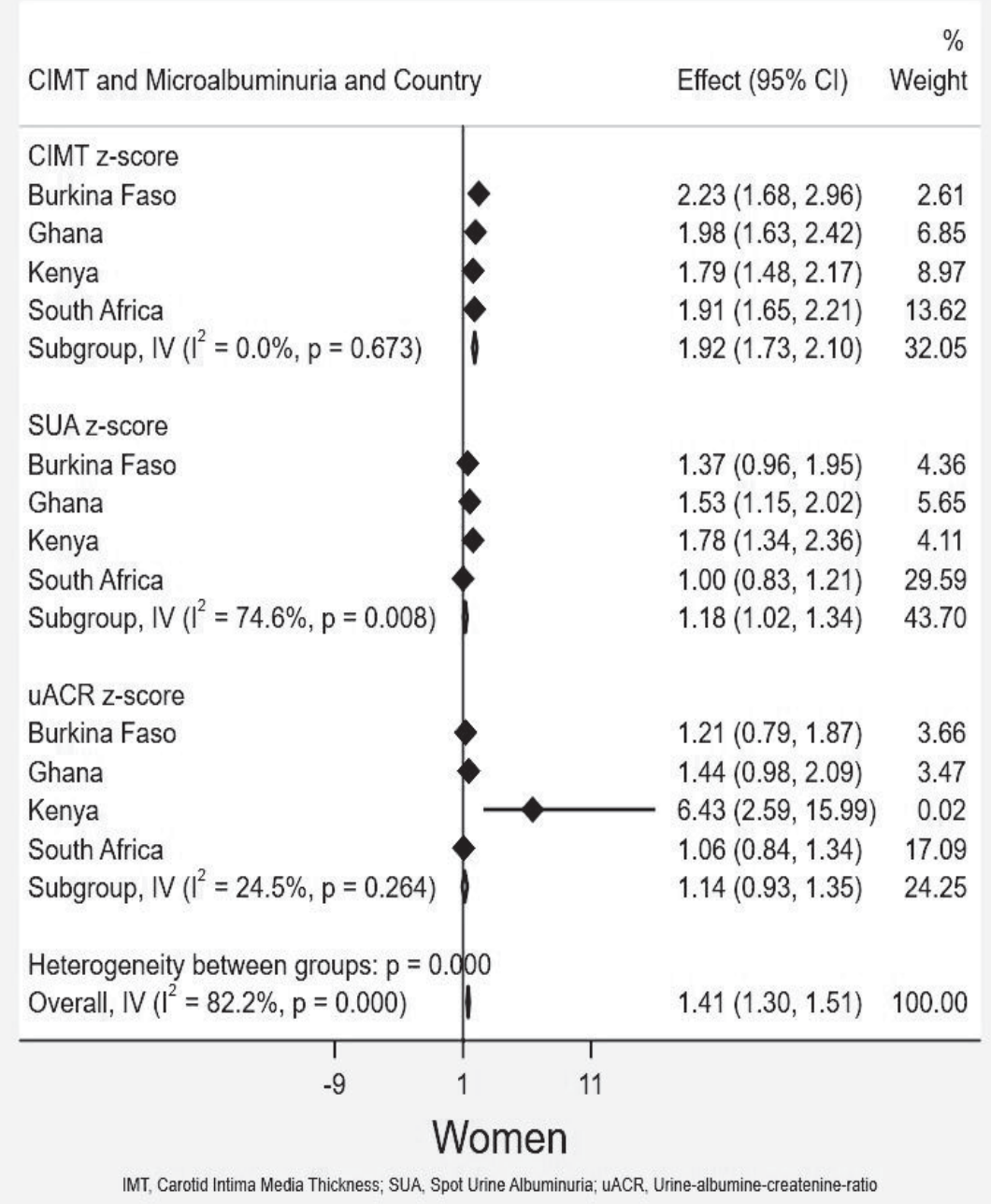

Figure 2: Meta-analyses of the comparative association of carotid atherosclerosis (CIMT) and microalbuminuria (SUA and UACR) with 10-year ASCVD risk in women from the four countries. CIMT, carotid intima-media thickness; IV, inverse variance; SUA, spot urine albuminuria and $\mathrm{UACR}$, urine albumin-creatinine-ratio 
$\%$

CIMT and Microalbuminuria and Country

Effect $(95 \% \mathrm{Cl}) \quad$ Weight

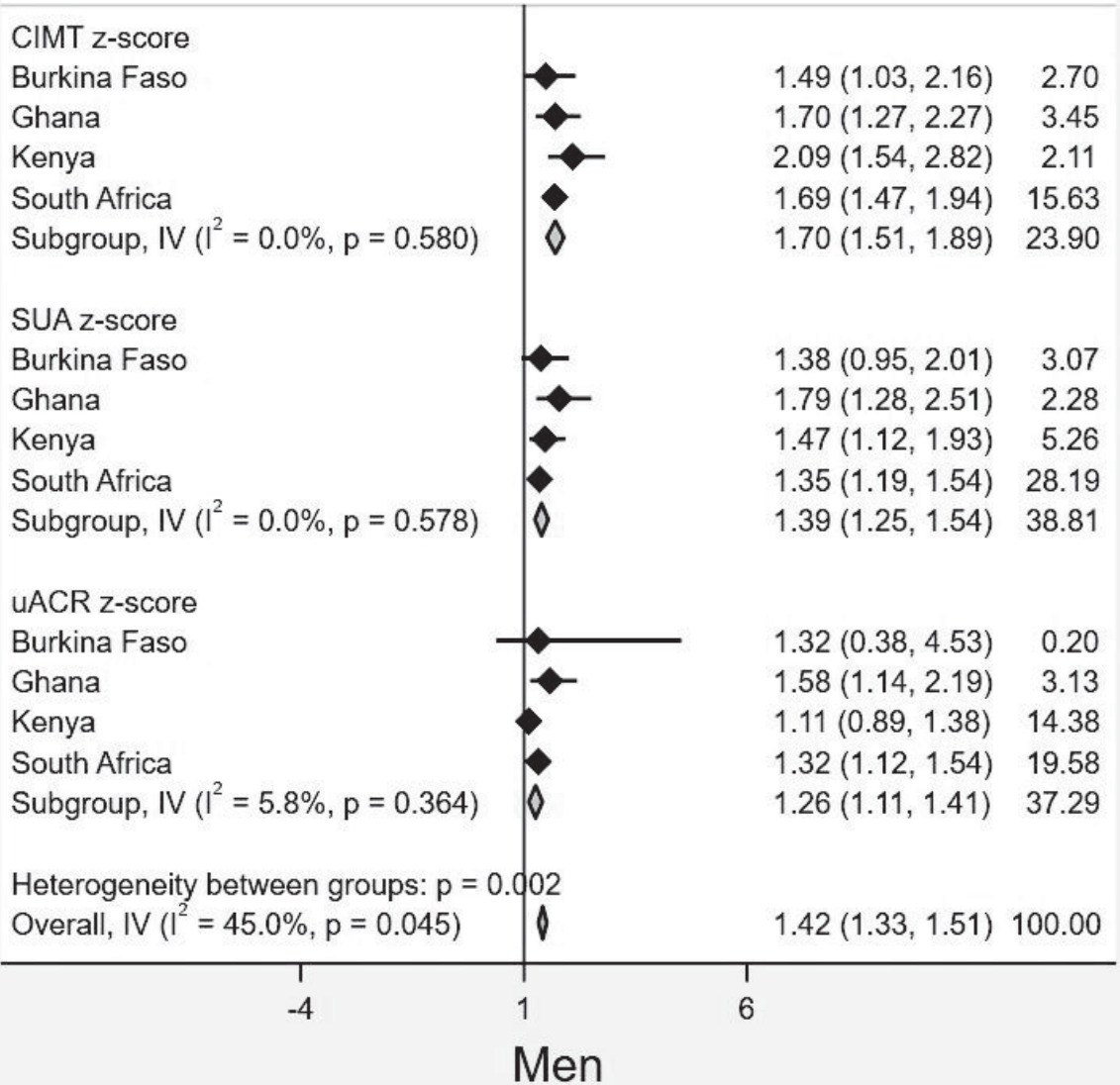

IMT, Carotid Intima Media Thickness; SUA, Spot Urine Albuminuria; UACR, Urine-albumine-createnine-ratio

Figure 3: Meta-analyses of the comparative association of carotid atherosclerosis (CIMT) and microalbuminuria (SUA and UACR) with 10-year ASCVD risk in men from the four countries. CIMT, carotid intima-media thickness; IV, inverse variance; SUA, spot urine albuminuria and $\mathrm{UACR}$, urine albumin-creatinine-ratio 


\section{Discussion}

This study determined the association between microalbuminuria (measured by SUA and UACR) and carotid atherosclerosis (as represented by CIMT) and their association with high 10-year ASCVD risk in Burkina Faso, Ghana, Kenya and South Africa. The present study demonstrated that microalbuminuria is associated with CIMT independent of the traditional CVD risk factors. We also demonstrated that carotid atherosclerosis and microalbuminuria are associated with high 10-year ASCVD risk in middle-aged adults.

We showed differences in the prevalence of carotid atherosclerosis and microalbuminuria between the various countries. Notably, participants from Kenya and South Africa reported high levels of microalbuminuria compared to those from Burkina Faso and Ghana while the opposite was observed for common CIMT. The CIMT results are consistent with a previous study involving this study population (27). The possible reason for the higher prevalence of microalbuminuria in Kenya and South Africa may be due to the higher prevalence of cardiometabolic diseases and HIV infection. The higher prevalence of alcohol use in Burkina Faso and Ghana may result in chronic liver disease and hence low circulatory serum albumin and creatinine levels. Using albumin-creatinine-ratio with a similar cut off to that used in the current study to define microalbuminuria, two studies involving HIV-exposed and HIVnaivve individuals conducted in rural South Africa and Hawaii observed HIV as an important predictor of microalbuminuria $(28,29)$. The pathophysiological basis for this observation involves HIV-nephropathy and HIV-associated immune complex kidney disease leading to increased leakiness of nephrons to molecules such as creatinine and albumin $(30,31)$.

We observed that independent of the classical CVD risk factors, SUA had a stronger association than UACR with CIMT. Some studies have reported an association between microalbuminuria (measured with urine albumin and ACR) and subclinical atherosclerosis independent of traditional CVD risk factors (3234). The underlying mechanisms for the observed association remain unclear. However, it has been reported that vascular endothelial damage may cause atherosclerosis and albuminuria $(10,35)$. Endothelial dysfunction has been shown to increase vascular leakiness in target vessels. If structural changes occur in the endothelium of the glomerular capillaries there may be increased permeability in the glomerular barrier leading to increased excretion of albumin into the urine (36). The non-significant association of UACR with CIMT warrants further investigation 
Carotid atherosclerosis and microalbuminuria were associated with high 10year ASCVD risk in women and men. Large epidemiological studies such as the Kuopio Ischaemic Heart Disease Risk Factor Study, Atherosclerosis Risk in Communities (ARIC) and the Cardiovascular Health Study have observed that increased CIMT was associated with a higher incidence of myocardial infarction and stroke independent of classical CVD risk factors (21,37-39). The Rotterdam and USE-IMT studies recently reported a significant association of CIMT with CVD and related risk factors $(40,41)$. While CIMT and microalbuminuria are strong independent predictors of cardiovascular disease, microalbuminuria is more apparent in individuals with hypertension and diabetes. Our data demonstrates that diabetes, use of anti-hypertensive medication and systolic blood pressure, which are each components of the PCE, are all higher in subjects with microalbuminuria as defined by either SUA or UACR. This is in line with previous studies that reported microalbuminuria was strongly associated with CVD in subjects with hypertension or diabetes (42). This was supported by an observational study that demonstrated that microalbuminuria occurs in approximately $5-15 \%$ of the general elderly population, in $11-17 \%$ of hypertensive individuals, and approximately $20-30 \%$ of individuals with type 2 diabetes (42). Among diabetics, biochemical alterations to the extracellular matrix may lead to glomerulosclerosis and premature atherosclerosis as well as insulin resistance leading to endothelial structural and functional damage which occurs relatively late in the atherothrombotic disease course and is strongly associated with advanced age (43).

Strengths and limitations: Our study presents with certain limitations such as the cross sectional design which prevents us from establishing causality. Social desirability and recall bias may also arise from the self-reported responses to certain questions such as educational status. The absence of clinical event data also affects the observed associations and hence the results must be interpreted with caution. These notwithstanding, there are a number of strengths of this study: the use of z-scores maximizes statistical power of the analyses and limits the non-centrality parameter of the statistic. We are thus able to compare the magnitude of the association of carotid atherosclerosis and microalbuminuria with 10-year CVD risk. The updated 2018 PCE equation has an improved accuracy among all races and sex subgroups (13). The use of a centralized laboratory to measure the biochemical measures and centralized training for the measurement of CIMT minimizes data variability. 


\section{Conclusions}

The presence of spot urine microalbuminuria may indicate risk of subclinical atherosclerosis and high 10-year ASCVD risk in middle-aged individuals from SSA. These data suggest that spot urine measures of albumin may be used within population studies to assess CVD risk in both genders and in the majority of countries included in this study. However, these data must be confirmed in longitudinal studies of cardiovascular events in African populations.

\section{Acknowledgement}

We would like to express our appreciation to the participants for their generosity and sacrifice in spending many hours responding to questionnaires, being measured, and having samples taken. We also acknowledge the remarkable contributions of our field workers, phlebotomists, laboratory scientists, administrators, data personnel, and other staff who contributed to the data and sample collections, processing, storage, and shipping.

\section{Funding}

This work was supported the Global Health Support Program of the University Medical Center Utrecht (UMCU), Utrecht University, the Netherlands, and the Navrongo Health Research Centre (NHRC), Ghana. The AWI-Gen Collaborative Centre is funded by the National Human Genome Research Institute (NHGRI), Office of the Director (OD); Eunice Kennedy Shriver National Institute Of Child Health \& Human Development (NICHD); the National Institute of Environmental Health Sciences (NIEHS), the Office of AIDS Research (OAR); and the National Institute of Diabetes and Digestive and Kidney Diseases (NIDDK), of the National Institutes of Health (award number U54HG006938) and its supplements, as part of the H3Africa Consortium. Additional funding was leveraged from the Department of Science and Technology, South Africa (award number DST/CON 0056/2014).

\section{Disclosure}

The authors have no conflict of interest to declare

\section{Author contribution}

EAN, NJC, KKG, ARO, MLB and DEG contributed to conception of the study. EAN, NJC, KKG and MLB designed the analyses plan. EAN and DB conducted the analyses and EAN drafted the manuscript with contributions from $D B, N J C$, $M L B, K K G, A R O$ and DEG. EAN, ARO, NJC and MR are investigators of the H3Africa AWI-Gen study and contributed to data collection and quality control. All authors critically reviewed and approved the final version of the manuscript for submission. 


\section{References}

1. Roth GA, Johnson C, Abajobir A, Abd-Allah F, Abera SF, Abyu G, et al. Global, Regional, and National Burden of Cardiovascular Diseases for 10 Causes, 1990 to 2015. J Am Coll Cardiol. 2017;70(1):1-25.

2. GBD 2015 Risk Factors Collaborators. Global, regional, and national comparative risk assessment of 79 behavioural, environmental and occupational, and metabolic risks or clusters of risks, 1990-2015: a systematic analysis for the Global Burden of Disease Study 2015. Lancet. 2016;388(10053):1659-724.

3. Yuyun MF, Sliwa K, Kengne AP, Mocumbi AO, Bukhman G. Cardiovascular Diseases in Sub-Saharan Africa Compared to High-Income Countries: An Epidemiological Perspective. Global Heart. 2020;15(1).

4. Gerstein HC, Mann JF, Yi Q, Zinman B, Dinneen SF, Hoogwerf B, et al. Albuminuria and risk of cardiovascular events, death, and heart failure in diabetic and nondiabetic individuals. . JAMA 2001;286: 421-6.

5. Libby P. Inflammation and Atherosclerosis. Circulation. 2002;105(9):1135-43.

6. Bots ML, Evans GW, Tegeler CH, Meijer R. Carotid Intima-media Thickness Measurements: Relations with Atherosclerosis, Risk of Cardiovascular Disease and Application in Randomized Controlled Trials. Chin Med J 2016;129(2):215-26.

7. Bots ML, Sutton-Tyrrell K. Lessons from the past and promises for the future for carotid intima-media thickness. J Am Coll Cardiol. 2012;60(17):1599-604.

8. Liviakis L, Pogue B, Paramsothy P, Bourne A, Gill EA. Carotid intima-media thickness for the practicing lipidologist. J Clin Lipidol. 2010;4(1):24-35.

9. Sung JK, Kim JY, Youn YJ, Lee JW, Ahn SG, Yoo BS, et al. Urine Albumin Creatinine Ratio is Associated with Carotid Atherosclerosis in a Community Based Cohort: Atherosclerosis Risk of Rural Area in Korean General Population Study. J Cardiovasc Ultrasound. 2010;18(4):134-8.

10. Stehouwer CD, Smulders YM. Microalbuminuria and risk for cardiovascular disease: Analysis of potential mechanisms. J Am Soc Nephrol. 2006;17(8):2106-11.

11. Yusuf S, Joseph P, Rangarajan S, Islam S, Mente A, Hystad P, et al. Modifiable risk factors, cardiovascular disease, and mortality in 155722 individuals from 21 highincome, middle-income, and low-income countries (PURE): a prospective cohort study. The Lancet. 2020;395(10226):795-808.

12. Ôunpuu S, Negassa A, Yusuf S. INTERHEART: A global study of risk factors for acute myocardial infarction. American Heart Journal. 2001;141(5):711-21.

13. Yadlowsky S, Hayward RA, Sussman JB, McClelland RL, Min YI, Basu S. Clinical Implications of Revised Pooled Cohort Equations for Estimating Atherosclerotic Cardiovascular Disease Risk. Ann Intern Med. 2018;169(1):20-9.

14. Ramsay M, Crowther NJ, Tambo E, Agongo G, Baloyi V, Dikotope S, et al. H3Africa AWIGen Collaborative Centre: a resource to study the interplay between genomic and environmental risk factors for cardiometabolic diseases in four sub-Saharan African countries. Global Health, Epidemiology and Genomics. 2016;1(e20):1-13. 
15. Ramsay M, Sankoh O, as members of the AWIGen study and the H3Africa Consortium. African partnerships through the H3Africa Consortium bring a genomic dimension to longitudinal population studies on the continent. Int J Epidemiol. 2016;45(2):305-8.

16. Ali SA, Soo C, Agongo G, Alberts M, Amenga-Etego L, Boua RP, et al. Genomic and environmental risk factors for cardiometabolic diseases in Africa: methods used for Phase 1 of the AWI-Gen population cross-sectional study. Glob Health Action. 2018;11(sup2):1507133.

17. Touboul PJ, Hennerici MG, Meairs S, Adams H, Amarenco P, Bornstein N, et al. Mannheim carotid intima-media thickness and plaque consensus (2004-2006-2011). An update on behalf of the advisory board of the 3rd, 4th and 5th watching the risk symposia, at the 13th, 15th and 20th European Stroke Conferences, Mannheim, Germany, 2004, Brussels, Belgium, 2006, and Hamburg, Germany, 2011. Cerebrovasc Dis. 2012;34(4):290-6.

18. Goff DC Jr, Lloyd-Jones DM, Bennett G, Coady S, D'Agostino RB Sr, Gibbons R, et al. 2013 ACC/AHA guideline on the assessment of cardiovascular risk: a report of the American College of Cardiology/American Heart Association Task Force on Practice Guidelines. J Am Coll Cardiol. 2014;1(63 (25 Pt B)):2935-59.

19. Naqvi TZ, Lee MS. Carotid intima-media thickness and plaque in cardiovascular risk assessment. JACC Cardiovasc Imaging. 2014;7(10):1025-38.

20. Bian L, Xia L, Wang Y, Jiang J, Zhang Y, Li D, et al. Risk Factors of Subclinical Atherosclerosis and Plaque Burden in High Risk Individuals: Results From a Community-Based Study. Front Physiol. 2018;9:739.

21. Chambless LE, Heiss G, Folsom AR, Rosamond W, Szklo M, Sharrett AR, et al. Association of coronary heart disease incidence with carotid arterial wall thickness and major risk factors: the Atherosclerosis Risk in Communities (ARIC) Study, 1987-1993. Am J Epidemiol. 1997;146(6):483-94.

22. Price JF, Tzoulaki I, Lee AJ, Fowkes FG. Ankle brachial index and intima media thickness predict cardiovascular events similarly and increased prediction when combined. J Clin Epidemiol. 2007;60(10):1067-75.

23. Derhaschnig U, Kittler $\mathrm{H}$, Woisetschläger $\mathrm{C}$, Bur A, Herkner $\mathrm{H}$, Hirschl MM. Microalbumin measurement alone or calculation of the albumin/creatinine ratio for the screening of hypertension patients? Nephrol Dial Transplant. 2002;17(1):81-5.

24. Halbesma N, Kuiken DS, Brantsma AH, Bakker SJ, Wetzels JF, De Zeeuw D, et al. Macroalbuminuria is a better risk marker than low estimated GFR to identify individuals at risk for accelerated GFR loss in population screening. J Am Soc Nephrol. 2006;17(9):2582-90.

25. Kidney Disease: Improving Global Outcomes (KDIGO) CKD Work Group. KDIGO 2012 Clinical Practice Guideline for the Evaluation and Management of Chronic Kidney Disease. Kidney International supplements. 2013;3(1):1-150.

26. Stevens PE, Levin A, for the Kidney Disease Improving Global Outcomes Chronic Kidney Disease Guideline Development Work Group Members. Evaluation and Management of Chronic Kidney Disease: Synopsis of the Kidney Disease: Improving Global Outcomes 2012 Clinical Practice Guideline. Ann Intern Med. 2013;158(11):825-30. 
27. George JA, Brandenburg J-T, Fabian J, Crowther NJ, Agongo G, Alberts $M$, et al. Kidney damage and associated risk factors in rural and urban sub-Saharan Africa (AWI-Gen): a cross-sectional population study. The Lancet Global Health. 2019;7(12):el632-e43.

28. Tongma C, Shikuma CM, Nakamoto BK, Kallianpur KJ, Umaki TM, Barbour JD, et al. Albuminuria as a Marker of Cardiovascular Risk in HIV-Infected Individuals Receiving Stable Antiretroviral Therapy. Hawaii J Med Public Health. 2103;72(9 Suppl 4):34-8.

29. Wensink GE, Schoffelen AF, Tempelman HA, Rookmaaker MB, Hoepelman Al, Barth RE. Albuminuria Is Associated with Traditional Cardiovascular Risk Factors and Viral Load in HIV-Infected Patients in Rural South Africa. PLoS One. 2015;10(8):e0136529.

30. Szczech LA, Grunfeld C, Scherzer R, Canchola JA, van der Horst C, Sidney S, et al. Microalbuminuria in HIV infection. AIDS. 2007;21(8):1003-9.

31. Hadigan C, Edwards E, Rosenberg A, Purdy JB, Fleischman E, Howard L, et al. Microalbuminuria in HIV disease. Am J Nephrol. 2013;37(5):443-51.

32. Kong $X$, Jia X, Wei Y, Cui M, Wang Z, Tang L, et al. Association between microalbuminuria and subclinical atherosclerosis evaluated by carotid artery intima-media in elderly patients with normal renal function. BMC Nephrology. 2012;13:37.

33. Jorgensen L, Jenssen T, Johnsen SH, Mathiesen EB, Heuch I, Joakimsen O, et al. Albuminuria as risk factor for initiation and progression of carotid atherosclerosis in non-diabetic persons: the Tromso Study. Eur Heart J. 2007;28(3):363-9.

34. Park HE, Heo NJ, Kim M, Choi SY. Significance of microalbuminuria in relation to subclinical coronary atherosclerosis in asymptomatic nonhypertensive, nondiabetic subjects. J Korean Med Sci. 2013;28(3):409-14.

35. Stehouwer CD. Endothelial dysfunction in diabetic nephropathy: state of the art and potential significance for non-diabetic renal disease. Nephrol Dial Transplant. 2004;19(4):778-81.

36. Deen WM. What determines glomerular capillary permeability? J Clin Invest 2004;114(10):1412-4.

37. Salonen R, Salonen JT. Determinants of carotid intima-media thickness: a populationbased ultrasonography study in eastern Finnish men. J Intern Med. 1991;229(3):225-31.

38. Chambless LE, Folsom AR, Clegg LX, Sharrett AR, Shahar E, Nieto FJ, et al. Carotid wall thickness is predictive of incident clinical stroke: the Atherosclerosis Risk in Communities (ARIC) study. Am J Epidemiol. 2000;151(5):478-87.

39. O'Leary DH, Polak JF, Kronmal RA, Manolio TA, Burke GL, Wolfson Jr SK. Carotid-artery intima and media thickness as a risk factor for myocardial infarction and stroke in older adults. Cardiovascular Health Study Collaborative Research Group. N Engl J Med. 1999;340(1):14-22.

40. Gijsberts CM, Groenewegen KA, Hoefer IE, Eijkemans MJ, Asselbergs FW, Anderson TJ, et al. Race/Ethnic Differences in the Associations of the Framingham Risk Factors with Carotid IMT and Cardiovascular Events. PLoS One. 2015;10(7):e0132321.

41. Iglesias del Sol A, Bots ML, Grobbee DE, Hofman A, Witteman JC. Carotid intima-media thickness at different sites: relation to incident myocardial infarction; The Rotterdam Study. Eur Heart J. 2002;23(12):934-40. 
42. Pollak J, Sypniewska G. Microalbuminuria and risk of cardiovascular diseases in patients with diabetes and hypertension. Biochemia Medica. 2007;18(1):25-34.

43. Damsgaard EM, Frøland A, Jørgensen OD, Mogensen CE. Prognostic value of urinary albumin excretion rate and other risk factors in elderly diabetic patients and nondiabetic control subjects surviving the first 5 years after assessment. Diabetologia. 1993;36:1030-6. 


\section{Supplementary material}

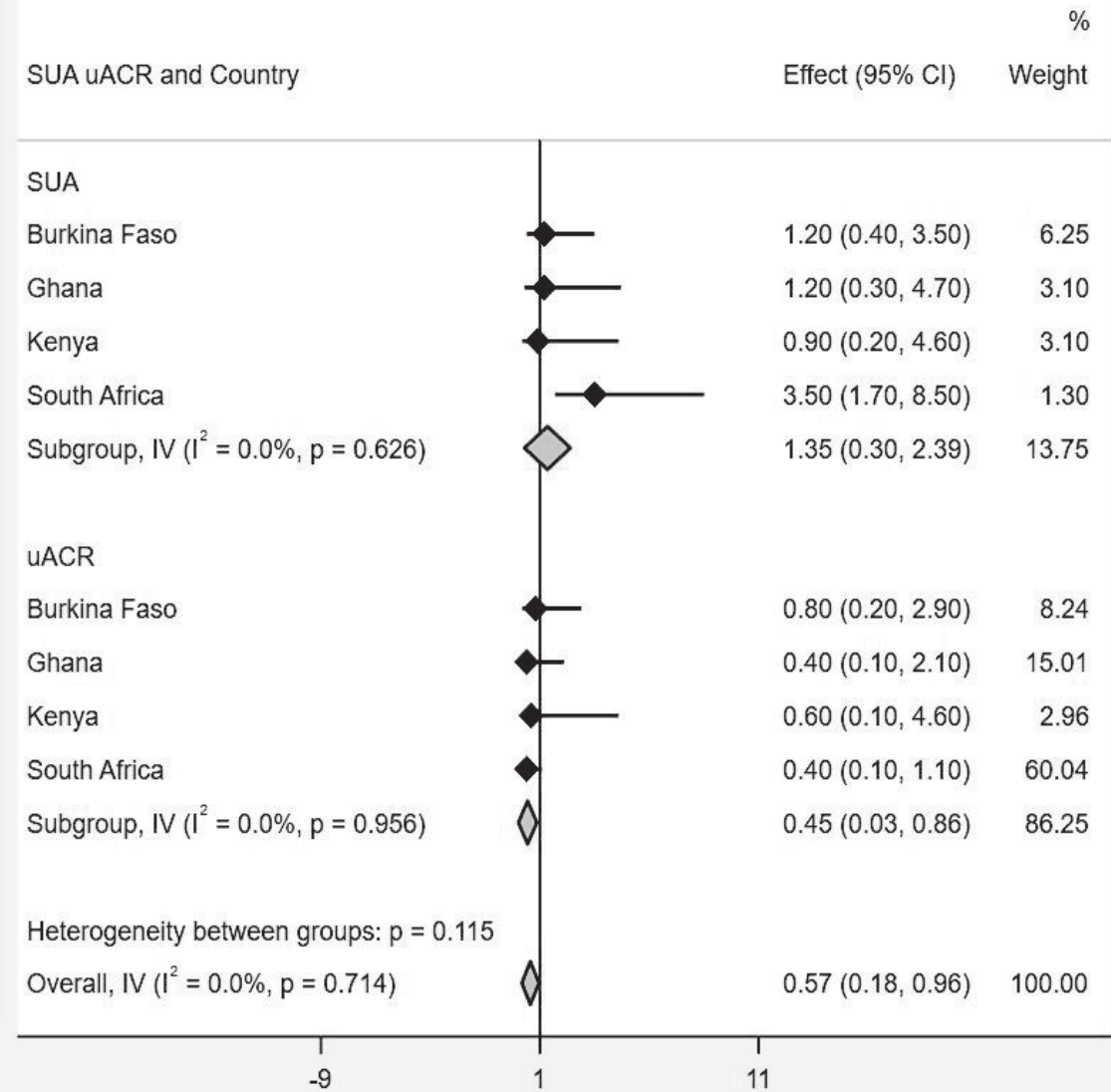

SUA, Spot Urine Albuminuria; UACR, Urine-albumine-createnine-ratio

Figure S1: Association of microalbuminuria (spot urine albuminuria and urine creatinine-albumin-ratio) with carotid intima-media thickness by the study countries; IV, inverse variance; SUA, spot urine albuminuria; $U A C R$, urine albumin-creatinine-ration 


\section{Contextualizing the evidence for CVD control}

In the past decade several global efforts have been initiated towards the prevention and management of non-communicable diseases (NCDs) which include cardiovascular disease (CVD). For the first time, NCDs were included in the Sustainable Development Goals (SDGs) agenda 2030 that was adopted in 2015 by the United Nations (1). Of the 17 SDGs, SDG3 focuses on health and commits governments to "ensure healthy lives and promote wellbeing for all ages". Specifically target 3.4 seeks to "reduce by one third premature mortality from NCDs through prevention and treatment, and promote mental health and wellbeing", which builds on the World Health Organization (WHO) "25x25" NCD target to reduce NCD mortality by $25 \%$ by the year 2025 (2-4). SDG target 3.5 focuses on the prevention and treatment of substance abuse, including harmful use of alcohol while 3.a seeks to strengthen the implementation of the WHO convention on tobacco control (1). Prevention of NCDs offers enormous gains since the major risk factors are also linked to many of the other SDGs such as poverty, nutrition, gender equality, energy, urbanization and climate.

Despite these efforts the burden of CVD continues to grow especially in sub-Saharan African countries. The lack of context specific research on the important risk factors for primary prevention may be a major contributor to the shortfalls in the global efforts. Investigating the burden and risk factors of carotid atherosclerosis among a population of Eastern, Western and southern Africans residing in sub-Saharan Africa (SSA), the H3Africa AWI-Gen study shows differences and similarities in risk of atherosclerotic CVD between these subregional blocks. A further comparison between African, African American, Asian, European and Hispanic populations highlights important race-ethnic similarities and differences in risk factors of carotid atherosclerosis. To our knowledge this is by far the largest pan-African study to investigate these differences using harmonized epidemiological data. This is also the first time, data from SSA populations are included in a pooled analyses for a race-ethnicity comparison of risk factors of carotid atherosclerosis.

In the current study, carotid intima-media thickness (CIMT) was used as a measure for atherosclerotic cardiovascular disease. We observed that the classical cardiovascular risk factors such as age, male gender, smoking, body mass index (BMI), systolic blood pressure, and low density lipoprotein (LDL) were associated with increased CIMT. High density lipoprotein (HDL), alcohol intake and physical activity were inversely associated with CIMT (5). We further highlight that in addition to BMI other adiposity measures, i.e. waist (WC) and hip circumference (HC), visceral (VAT) and subcutaneous adipose tissue (SCAT) were associated with CIMT. Specifically, BMI had the greatest positive association followed by VAT, WC and HC whilst SCAT had an inverse 
association with CIMT. For the study populations in Burkina Faso and Ghana an inverse association of VAT with CIMT was observed (6). An unexpected finding was the inverse association of HIV with CIMT in this sub-Saharan population. In comparison with other populations, Africans and African Americans had a lower effect of LDL on CIMT compared to Europeans. The protective effect of $\mathrm{HDL}$ and alcohol on carotid atherosclerosis was higher in people of African ancestry (African and African Americans) compared to Europeans (7). The thesis further established that a simple screening tool such as the Cardiovascular Health Index proposed by the American Heart Association (AHA) is a strong predictor of carotid atherosclerosis in African populations (8). Finally, the thesis demonstrated that measures of microalbuminuria correlated positively with carotid atherosclerosis and highlights the strong magnitude of the association of carotid atherosclerosis with high 10-year CVD risk (9).

Our observed association of the classical CVD risk factors with CIMT is paralleled by reports from the INTERHEART (10) and the PURE (11) studies which identified these factors as major contributors to myocardial infarction and stroke. The USE-IMT collaboration, also using CIMT, further established these risk factors in African American, Asian, European and Hispanic populations $(12,13)$. Consistency in the higher risk of CVD conferred by age and sex (men than women) has important implications for mapping out targeted CVD screening programs and management.

The differing burden of classical cardiovascular risk factors within different populations across Africa is possibly driven by the level of epidemiological transition present in each population, and has been highlighted in this thesis and elsewhere. Thus, we observed that lower risk factor burden was observed in rural areas of Nanoro in Burkina Faso and Navrongo in Ghana when compared to peri-urban Dikgale and Agincourt in South Africa and to more urban areas such as Nairobi in Kenya and Soweto in South Africa (14). It has been established that the stage of epidemiological transition is a major contributor to differences in CVD risk in various populations $(15,16)$. It is also important to note that within the same country the burden of CVD risk factors may differ, particularly between rural and urban populations. This was observed in South Africa where periurban Agincourt and Dikgale had a lower burden of risk factors compared to urban Soweto $(17,18)$. In addition to variability in the geographical distribution of traditional CVD risk factors, variability in the association of novel risk factors with subclinical CVD highlight the need for more in-depth studies across the African continent (9).

Sex related differences in CVD morbidity and risk factors are reported in the literature. African women, just like African American women (19) were reported 
to have a higher prevalence of obesity compared to their male counterparts (6) and this is also reflected in other studies from Africa and other low- and middle-income countries (LMIC) (20-22). This thesis has also made similar observations where obesity is more common in women than in men. Several studies have reported a poor perception of the threat of overweight and obesity to health among African overweight/obese women (23-25). Further to this the social desirability for a larger body size among women has been reported in some African communities $(26,27)$. This context has implications in crafting policies to control obesity among women in African populations.

The contribution of infectious diseases such as HIV, tuberculosis and malaria to the pathophysiology of CVDs in SSA has been described in the literature (28-30). It has been widely reported in Europe and North America that HIV is associated with an increased rate of preclinical atherosclerosis and CVD risk and events. This was largely due to the chronic inflammatory states and immune activation leading to atherosclerosis $(31,32)$. The use of antiretroviral therapy (ART) thus controls the inflammatory process leading to reduced viraemia and atherosclerosis process (5). For African populations, however, we and others have observed lower prevalence of diabetes and hypertension among people living with HIV (PLWHIV) (33-35), favourable CVD risk profile among HIV positive compared to HIV negative people (35), and an inverse association of HIV with atherosclerosis (5). In addition, a recent study from rural Uganda observed that the progression of carotid atherosclerosis was no different between HIV positive compared to HIV negative people (29). The reason for the reduced levels of CVD risk factors in PLWHIV in SSA is not fully understood, however, one possible explanation is greater healthcare utilization among PLWHIV as well as heightened screening and management of CVD-related co-morbidities in this population, but this requires further investigation. There is however an emerging trend of increased risk of obesity associated with the use of newer anti-retroviral agents such as dolutegravir (36). This observation should serve as a basis for further studies on drug discovery and mapping out of strategies for early detection and management of CVD risk factors with behavioural change interventions.

Drawing on the infrastructure put in place to develop an efficient HIV care cascade, attempts should be made to use this system for the screening and management of CVD risk factors (37). This we believe will ensure a sustainable way of managing CVD within existing systems without necessarily investing in or establishing new systems. This is advantageous in an era where healthcare funding for CVD care in SSA is already inadequate with communicable diseases taking a much higher priority in healthcare expenditure. 
Differences observed in the current study in the association of CVD risk factors with CIMT across race-ethnic groups may be linked to genetic factors. Thus, this thesis and other literature highlight the negative association of alcohol with CIMT, healthy lipid profiles (5) and reduced atherosclerosis-related inflammatory markers among Africans and African Americans may result from specific gene variants in these populations (38-40). In this thesis, African Americans and West Africans had the highest CIMT levels (7) when compared to the other African populations. This is interesting because African American ancestral populations are thought to originate from west and central Africa (41, 42). Thus, it is possible that distinct gene polymorphisms related to high CIMT in the West African population may partially contribute to high CIMT levels in African American subjects. Obviously, this hypothesis must be tested with in depth comparative gene association studies in both populations.

\section{Control of CVD - maximizing efforts at a minimal cost}

The current thesis offers evidence on contextual disparities and similarities in CVD risk among African, African American, Asian, European and Hispanic populations (7). It further highlights key priority CVD risk factors (hypertension and obesity) in Eastern, Western and Southern SSA based on the magnitude of the observed associations of these factors to CIMT, and their high prevalence. The region is unprepared for the growing burden of coronary artery disease and cerebrovascular events, as there is evidence of insufficient health care infrastructure and resources as well as serious deficiencies in the numbers of cardiac specialists to combat CVDs in this region $(43,44)$. Appropriate interventions should therefore be context specific due to the low budgetary allocations to NCDs with prevailing communicable and maternal and child health related morbidities being prioritised.

Considering the numerous challenges and scarce resources in implementing interventions to control CVDs, extrapolating interventions from other settings to Africa may not be appropriate. To overcome these resource constraints, it is important to formulate strategies based on context specific evidenced based research. Following identification of the important risk factors a multisectoral approach to enhance both treatment and prevention is vital taking into account sociocultural factors, infrastructure levels and financial limitations.

Given the relatively short time frame to meet the current global sustainable development goals and other set goals, efforts will have to include both population-based strategies as well as strengthening health service access and quality for the delivery of primary and secondary prevention as well as acute management. 
Due to low national budgetary allocations for the management of CVD (43), pragmatic health service solutions through the use of existing health infrastructure will be a major boost to chronic disease identification and care. Health system strengthening through the use of current primary health care modules in various SSA countries is an important step. For instance, existing health care workers and trained community health volunteers could be further trained to promptly screen and identify individuals at a higher risk of CVD. They could also be trained to offer counseling aimed at risk factor reduction. In some African settings it has been demonstrated that nurses and health volunteers at the primary healthcare level have been a useful and effective means of offering basic treatment and monitoring of hypertension and diabetes (37). Valuable lessons can also be learnt from the WHO HEARTS Package which supports Ministries of Health to strengthen CVD management in primary health care settings (45). HEARTS can easily be adopted at the primary healthcare level to target identified CVD risk factors and implement evidence-based strategies for managing CVD.

The major financial commitments to these low budget solutions will include provision of screening tools such as weight scales, blood pressure machines, and point-of-care glucometers and lipid measuring devices. Effective sustained and continuous training of primary healthcare workers as well as health volunteers on the generated protocols may require minimal funding to achieve desired results. These blended pragmatic steps are sure to assist in risk factor reduction, early detection and improve adherence to the treatment of CVD. The above points are supported propositions in the SDGs. The SDG 3.8 echoes the need to achieve universal health coverage, including financial risk protection, access to high quality health-care services and access to safe, effective and affordable essential medicines and vaccines for all (1). This is further supported by 3.b which is aimed at supporting the research and development of vaccines and medicines for communicable and non-communicable diseases that primarily affect LMIC countries, and to provide access to affordable essential medicines and vaccines (1).

This PhD thesis is based on the largest study from SSA to date with harmonized training of researchers regarding data collection methodologies and centralized analysis of the serum biomarkers of CVD risk. The coefficient of variation for most of the anthropometric, ultrasound and serum biochemical measures was maintained at less than $2 \%$ thus measurement variability in most of the study parameters was minimized. The study also included populations from across SSA (Eastern, Western and Southern SSA) within countries at different levels of the epidemiological transition thus providing the study with a broad numeric range for each of the socio-demographic and cardiometabolic variables 
and highlighting the heterogeneous nature of African populations. This is an important feature of the study and demonstrates that a 'one size fits all' approach to the development of population-based screening and interventions for CVD may not be appropriate. Potential limitations of the study include the observed association of HIV with CIMT in West Africa, which may be biased due to the self-reported assessment of HIV in Burkina Faso and Ghana. However, we believe the extent of the bias is not sufficient to affect the results due to the very low prevalence of HIV infection in the affected West African sites, which is supported by data from the literature (5). The use of CIMT as a proxy marker for CVD may influence the observations made and hence interpretation of the results. However, we believe the evidence from the literature on the association of CIMT with CVD (46) strongly supports our use of this marker.

\section{Future research}

Finally, further research in the form of longitudinal studies to establish key risk factors associated with clinical cardiovascular events are much needed. For instance, the role of visceral adiposity in CVD risk in West African populations needs further validation. Longitudinal research will serve as a good avenue to establish Afrocentric reference values for several of the metabolic risk factors. With several large population-based studies such as the H3Africa AWI-Gen study transitioning into longitudinal cohorts, it may be prudent for researchers to prioritize the collection of clinical event data. The reason for the higher CIMT in West African compared to Eastern and Southern African population may be explained by unmeasured risk factors. Further research may therefore be needed to understand this observation. It will also be useful for a future individual participant data meta-analysis to look at the contribution of HIV and ART use to CVD risk among PLWHIV in sub-Saharan Africa. Exposure adversity (such as poor nutrition) in the early life stages has been shown to result in increased risks for obesity, diabetes and hypertension in later life (47-49). This may partly explain the higher burden of CVD risk among young people in Africa compared to other developed nations. It would therefore be important if future studies could focus on the effect of adverse exposure to infectious agents and malnutrition as predictors of early onset atherosclerosis and cardiovascular risk factors.

\section{Conclusion}

Classical cardiovascular risk factors are associated with atherosclerotic cardiovascular diseases in African populations. The emerging trend of increasing obesity in African populations has the potential of increasing the risk of myocardial infarctions and cerebrovascular events. Compared to other race-ethnicities, African population could benefit from interventions 
that promote healthy diet (plant source protein) and healthy lipid profiles as a measure to control CVD. Carotid atherosclerosis (carotid intima-media thickness) and microalbuminuria (spot urine albuminuria and urine albumincreatinine-ratio) are associated with atherosclerotic cardiovascular diseases, hence simple spot urine albuminuria should give a heightened suspicion for onset of CVD. Therefore, simple screening methods such as the use of urine dipstick can safely be used at the primary healthcare setup to identify at risk individuals. The association of cardiovascular health index with atherosclerosis offers an important message in mapping out measures to control the classical cardiovascular risk factors. Particularly the control of smoking, obesity, hypertension and improving physical activities levels are essential in controlling CVD in African populations. Population level strategies could include banning of smoking especially in public places and creating smoking zones. Similarly legal frameworks could adopt reductions in the content of salt in bread and sugar in beverages or imposition of taxes on companies to reduce the content of salt and sugar in their products. There is evidence of the effectiveness of this in high and middle-income countries $(50,51)$. A review across seven subSaharan African countries showed willingness of the countries to adopt Sugar Sweetened Beverage (SSB) tax as a means to control NCDs at a population level (52). Promoting health screening through the adoption of annual days for the screening of risk factors for CVD may be useful avenues to identify undiagnosed cases of hypertension, diabetes or dyslipidaemia. However, such programs will need to be tailored to the populations under investigation, as the current study does highlight the heterogeneous socio-demographic and cardiometabolic features of countries across SSA.

Finally, a blended approach of population level policies such as taxation, ban on smoking indoors and health system approaches such as implementing WHO HEARTS package in existing primary health care systems will make a huge impact in the control of CVDs and associated risk factors. 


\section{Key messages}

- It is important to expand the scope of research on the association of carotid intima-media thickness with cardiovascular disease (CVD) in African populations through the use of longitudinal studies and the collection of data on clinical events.

- The classical cardiovascular risk factors are important risk factors in African populations.

- The relative importance of one risk factor over the other based on the magnitude of its effect on atherosclerotic cardiovascular disease is an important consideration in adopting control measures in different populations.

- The use of existing infrastructure for HIV screening and treatment within primary healthcare systems in sub-Saharan Africa is one unique approach to preventing CVD by controlling risk factor burden. These systems will serve as a good foundation for screening for CVD risk factors and treatment monitoring at the primary healthcare level

- Further to this other primary healthcare set-ups such as community health and planning services (CHPS) in Ghana, which are meant for maternal and child health care services could incorporate the screening of CVD risk factors into their routine services within the community. 


\section{References}

1. United Nations. A/RES/70/1-Transforming our world: the 2030 Agenda for Sustainable Development. https://www.un.org/ga: United Nations General Assembly; Seventieth session; 2015.

2. WHO. Global action plan for the prevention and control of noncommunicable diseases 2013-2020. World Health Organization, 20 Avenue Appia, 1211 Geneva 27, Switzerland; 2013.

3. Dugani S, Gaziano TA. 25 by 25 : Achieving Global Reduction in Cardiovascular Mortality. Curr Cardiol Rep. 2016;18(1):10.

4. WHO. Global status report on noncommunicable diseases 2014. WHO Press, World Health Organization, 20 Avenue Appia, 1211 Geneva 27, Switzerland WHO; 2014.

5. Nonterah EA, Boua PR, Klipstein-Grobusch K, Asiki G, Micklesfield LK, Agongo G, et al. Classical cardiovascular risk factors and HIV are associated with carotid intimamedia thickness in adults from sub-Saharan Africa: Findings from H3Africa AWI-Gen Study. J Am Heart Assoc. 2019;8(14).

6. Nonterah EA, Agongo G, Oduro A, Soo CC, Micklesfield LK, et al. Adiposity Phenotypes and Subclinical Atherosclerosis in Adults from Sub-Saharan Africa: An H3Africa AWIGen Study. Global Heart. 2021;16(1).

7. Nonterah EA, Crowther NJ, Klipstein-Grobusch K, Oduro AR, Kavousi M, Agongo G, et al. Race-ethnic differences in the association between classical cardiovascular risk factors and common carotid intima-media thickness: an individual participant data meta-analysis. Submitted to Circulation. 2021.

8. Nonterah EA, Crowther NJ, Oduro A, Agongo G, Micklesfield LK, Boua PR, et al. Poor cardiovascular health is associated with subclinical atherosclerosis in apparently healthy sub-Saharan African populations: an H3Africa AWI-Gen study. BMC Medicine. 2021;19(1).

9. Nonterah EA, Boateng D, Crowther NJ, Klipstein-Grobusch K, Oduro AR, Agongo G, et al. Carotid atherosclerosis, microalbuminuria and predicted 10-year cardiovascular disease risk in sub-Saharan Africa. Submitted to atherosclerosis. 2021.

10. Yusuf S, Hawken S, Ôunpuu S, Dans T, Avezum A, Lanas F, et al. Effect of potentially modifiable risk factors associated with myocardial infarction in 52 countries (the INTERHEART study): case-control study. The Lancet. 2004;364(9438):937-52.

11. Yusuf S, Joseph P, Rangarajan S, Islam S, Mente A, Hystad P, et al. Modifiable risk factors, cardiovascular disease, and mortality in 155722 individuals from 21 highincome, middle-income, and low-income countries (PURE): a prospective cohort study. The Lancet. 2020;395(10226):795-808.

12. Wang $X$, Dalmeijer GW, den Ruijter HM, Anderson TJ, Britton AR, Dekker J, et al. Clustering of cardiovascular risk factors and carotid intima-media thickness: The USE-IMT study. PLoS One. 2017;12(3):e0173393. 
13. Gijsberts CM, Groenewegen KA, Hoefer IE, Eijkemans MJ, Asselbergs FW, Anderson TJ, et al. Race/Ethnic Differences in the Associations of the Framingham Risk Factors with Carotid IMT and Cardiovascular Events. PLoS One. 2015;10(7):e0132321.

14. Ali SA, Soo C, Agongo G, Alberts M, Amenga-Etego L, Boua RP, et al. Genomic and environmental risk factors for cardiometabolic diseases in Africa: methods used for Phase 1 of the AWI-Gen population cross-sectional study. Glob Health Action. 2018;11(sup2):1507133.

15. Roth GA, Forouzanfar MH, Moran AE, Barber R, Nguyen G, Feigin VL, et al. Demographic and epidemiologic drivers of global cardiovascular mortality. $\mathrm{N}$ Engl J Med. 2015;372(14):1333-41.

16. Defo BK. Demographic, epidemiological, and health transitions: are they relevant to population health patterns in Africa? Glob Health Action. 2014;7:22443.

17. Gomez-Olive FX, Ali SA, Made F, Kyobutungi C, Nonterah E, Micklesfield L, et al. Regional and Sex Differences in the Prevalence and Awareness of Hypertension: An H3Africa AWI-Gen Study Across 6 Sites in Sub-Saharan Africa. Glob Heart. 2017;12(2):81-90.

18. Ramsay M, Crowther NJ, Agongo G, Ali SA, Asiki G, Boua RP, et al. Regional and sexspecific variation in BMI distribution in four sub-Saharan African countries: The H3Africa AWI-Gen study. Glob Health Action. 2018;11(sup2):1556561.

19. Cossrow N, Falkner B. Race/ethnic issues in obesity and obesity-related comorbidities. J Clin Endocrinol Metab. 2004;89(6):2590-4.

20. Nonterah EA, Debpuur C, Agongo G, Amenga-Etego L, Crowther NJ, Ramsay M, et al. Socio-demographic and behavioural determinants of body mass index among an adult population in rural Northern Ghana: the AWI-Gen study. Glob Health Action. 2018;11(sup2):1467588.

21. NCD Risk Factor Collaboration (NCD-RisC) - Africa Working Group. Trends in obesity and diabetes across Africa from 1980 to 2014: an analysis of pooled populationbased studies. Int J Epidemiol. 2017;46(5):1421-32.

22. NCD Risk Factor Collaboration (NCD-RisC). Rising rural body-mass index is the main driver of the global obesity epidemic in adults. Nature 2019;569(7755):260-4.

23. Okop KJ, Mukumbang FC, Mathole T, Levitt N, Puoane T. Perceptions of body size, obesity threat and the willingness to lose weight among black South African adults: a qualitative study. BMC Public Health. 2016;16:365.

24. Puraikalan Y. Perceptions of Body Image and Obesity among Cross Culture: A Review. Obesity Research - Open Journal. 2018;5(1):1-4.

25. Shoneye C, Johnson F, Steptoe A, Wardle J. A qualitative analysis of black and white British women's attitudes to weight and weight control. J Hum Nutr Diet. 2011;24(6):53642.

26. Benkeser RM, Biritwum R, Hill AG. Prevalence of Overweight and Obesity and Perception of Healthy and Desirable Body Size in Urban, Ghanaian Women. Ghana Medical Journal. 2012;46(2):66-75.

27. Micklesfield LK, Lambert EV, Hume DJ, Chantler S, Pienaar PR, Dickie K, et al. Sociocultural, environmental and behavioural determinants of obesity in black South African women. Cardiovasc J Afr. 2013;24(9-10):369-75. 
28. Wong EB, Olivier S, Gunda R, Koole O, Surujdeen A, Gareta D, et al. Convergence of infectious and non-communicable disease epidemics in rural South Africa: a cross-sectional, population-based multimorbidity study. The Lancet Global Health. 2021;9(7):e967-e76.

29. Siedner MJ, Bibangambah P, Kim JH, Lankowski A, Chang JL, Yang IT, et al. Treated HIV Infection and Progression of Carotid Atherosclerosis in Rural Uganda: A Prospective Observational Cohort Study. J Am Heart Assoc. 2021;10(12):e019994.

30. Basham CA, Smith SJ, Romanowski K, Johnston JC. Cardiovascular morbidity and mortality among persons diagnosed with tuberculosis: A systematic review and meta-analysis. PLoS One. 2020;15(7):e0235821.

31. Shah ASV, Stelzle D, Lee KK, Beck EJ, Alam S, Clifford S, et al. Global Burden of Atherosclerotic Cardiovascular Disease in People Living With HIV. Circulation. 2018;138(11):1100-12.

32. So-Armah K, Benjamin LA, Bloomfield GS, Feinstein MJ, Hsue P, Njuguna B, et al. HIV and cardiovascular disease. The Lancet HIV. 2020;7(4):e279-e93.

33. Manne-Goehler J, Montana L, Gomez-Olive FX, Rohr J, Harling G, Wagner RG, et al. The ART Advantage: Health Care Utilization for Diabetes and Hypertension in Rural South Africa. J Acquir Immune Defic Syndr. 2017;75(5):561-7.

34. Manne-Goehler J, Siedner MJ, Montana L, Harling G, Geldsetzer P, Rohr J, et al. Hypertension and diabetes control along the HIV care cascade in rural South Africa. $J$ Int AIDS Soc. 2019;22(3):e25213.

35. Vos AG, Barth RE, Klipstein-Grobusch K, Tempelman HA, Deville WLJ, Dodd C, et al. Cardiovascular Disease Burden in Rural Africa: Does HIV and Antiretroviral Treatment Play a Role?: Baseline Analysis of the Ndlovu Cohort Study. J Am Heart Assoc. 2020;9(7):e013466.

36. Venter WDF, Sokhela S, Simmons B, Moorhouse M, Fairlie L, Mashabane N, et al. Dolutegravir with emtricitabine and tenofovir alafenamide or tenofovir disoproxil fumarate versus efavirenz, emtricitabine, and tenofovir disoproxil fumarate for initial treatment of HIV-1 infection (ADVANCE): week 96 results from a randomised, phase 3, non-inferiority trial. The Lancet HIV. 2020;7(10):e666-e76.

37. Haykin LA, Francke JA, Abapali A, Yakubu E, Dambayi E, Jackson EF, et al. Adapting a nurse-led primary care initiative to cardiovascular disease control in Ghana: a qualitative study. BMC Public Health. 2020;20(1).

38. Bierut LJ, Goate AM, Breslau N, Johnson EO, Bertelsen S, Fox L, et al. ADHIB is associated with alcohol dependence and alcohol consumption in populations of European and African ancestry. Mol Psychiatry. 2012;17(4):445-50.

39. Crabb DW, Matsumoto M, Chang D, You M. Overview of the role of alcohol dehydrogenase and aldehyde dehydrogenase and their variants in the genesis of alcohol-related pathology. Proc Nutr Soc. 2004;63(1):49-63.

40. Fuchs FD, Chambless LE, Folsom AR, Eigenbrodt ML, Duncan BB, Gilbert A, et al. Association between alcoholic beverage consumption and incidence of coronary heart disease in whites and blacks: the Atherosclerosis Risk in Communities Study. Am J Epidemiol. 2004;160(5):466-74. 
41. Micheletti SJ, Bryc K, Ancona Esselmann SG, Freyman WA, Moreno ME, Poznik GD, et al. Genetic Consequences of the Transatlantic Slave Trade in the Americas. Am J Hum Genet. 2020;107(2):265-77.

42. Zakharia F, Basu A, Absher D, Assimes TL, Go AS, Hlatky MA, et al. Characterizing the admixed African ancestry of African Americans. Genome Biol. 2009;10(12):RI41.

43. Yuyun MF, Sliwa K, Kengne AP, Mocumbi AO, Bukhman G. Cardiovascular Diseases in Sub-Saharan Africa Compared to High-Income Countries: An Epidemiological Perspective. Global Heart. 2020;15(1).

44. Gupta R, Yusuf S. Challenges in management and prevention of ischemic heart disease in low socioeconomic status people in LLMICs. BMC Med. 2019;17(1):209.

45. WHO. HEARTS Technical package for cardiovascular disease management in primary health care: systems for monitoring. . Geneva: World Health Organization; 2018 (WHO/ $\mathrm{NMH} / \mathrm{NVI} / 19.8)$. Contract No.: Licence: CC BY-NC-SA 3.0 IGO.

46. Bots ML, Evans GW, Tegeler CH, Meijer R. Carotid Intima-media Thickness Measurements: Relations with Atherosclerosis, Risk of Cardiovascular Disease and Application in Randomized Controlled Trials. Chin Med J 2016;129(2):215-26.

47. Godfrey KM, Fleming TP, Whitelaw E, Garrick D, McConnell JML, Heindel JJ, et al. Developmental Origins of Health and Disease. Cambridge University Press (CUP): Cambridge, UK; 2006.

48. De Rooij SR, Bleker LS, Painter RC, Ravelli AC, Roseboom TJ. Lessons learned from 25 Years of Research into Long term Consequences of Prenatal Exposure to the Dutch famine 1944-45: The Dutch famine Birth Cohort. Int J Environ Health Res. 2021:1-15.

49. Norris SA, Daar A, Balasubramanian D, Byass P, Kimani-Murage E, Macnab A, et al. Understanding and acting on the developmental origins of health and disease in Africa would improve health across generations. Glob Health Action. 2017;10(1):1334985.

50. Schwendicke F, Stolpe M. Taxing sugar-sweetened beverages: impact on overweight and obesity in Germany. BMC Public Health. 2017;17(1):88.

51. Park H, Yu S. Policy review: Implication of tax on sugar-sweetened beverages for reducing obesity and improving heart health. Health Policy and Technology. 2019;8(1):92-5.

52. Thow AM, Abdool Karim S, Mukanu MM, Ahaibwe G, Wanjohi M, Gaogane L, et al. The political economy of sugar-sweetened beverage taxation: an analysis from seven countries in sub-Saharan Africa. Glob Health Action. 2021;14(1):1909267. 


\section{Summary}

The burden of atherosclerotic cardiovascular diseases in sub-Saharan Africa (SSA) is rising steadily and global efforts to reduce cardiovascular disease morbidity and mortality require the use of context specific interventions. The evidence guiding formulations of policies require pragmatic research taking note of local context that influences etiological relationships of risk factors with cardiovascular disease (CVD).

A general background to the study was discussed with chapter 1 providing an in-depth discussion on the global burden of CVD and the rising burden in the SSA region. We observed the rising burden in SSA to be largely due to improving life expectancies and the rise in CVD risk factors. The chapter also discussed the use of carotid-intima media thickness (CIMT) as a marker for cardiovascular disease.

Part 1 of the thesis assessed the risk factors for increased CIMT in both SSA and other populations.

In chapter 2 we determined the association of the classical cardiovascular risk factors and HIV with CIMT. The 8872 adults $(50.8 \%$ men) had a mean age of $50 \pm 6$ years with age- and sex-adjusted mean ( \pm SE) CIMT of $640 \pm 23 \mu \mathrm{m}$. Participants from Ghana and Burkina Faso had higher CIMT compared with other sites. Age $(\beta=6.77,95 \% \mathrm{Cl}[6.34,7.19] \mu \mathrm{m})$, body mass index (17.6 [12.5, $22.8] \mu \mathrm{m})$, systolic blood pressure $(7.52[6.21,8.83] \mu \mathrm{m})$, low-density lipoprotein cholesterol $(5.08[2.10,8.06] \mu \mathrm{m})$ and male gender $(10.3[4.75,15.9] \mu \mathrm{m})$ were associated with higher CIMT. Smoking was associated with higher CIMT in men. High-density lipoprotein cholesterol $(-12.2[-17.9,-6.41] \mu \mathrm{m})$ and alcohol consumption $(-13.5[-19.1,-7.91] \mu \mathrm{m})$ were inversely associated with CIMT. HIV was not significantly associated with CIMT $(26.80(-41.40,95.01) \mu \mathrm{m} ; \mathrm{p}=0.441)$. Given the rising prevalence of cardiovascular disease risk factors in SSA, atherosclerotic diseases may become a major pan-African epidemic unless preventive measures are taken particularly for the prevention of hypertension, obesity, and smoking. HIV-specific studies are needed to fully understand the association between HIV and CIMT in SSA.

In response to the rising prevalence of obesity in SSA, chapter 3 determined the association between various adiposity phenotypes and CIMT in 9010 middle aged adults from SSA. Men had higher levels of visceral fat (VAT) than women while women had higher BMI, waist (WC) and hip circumference (HC) and subcutaneous fat (SCAT) than men at all sites except Burkina Faso. In the pooled analyses, BMI ( $\beta$-value $[95 \% \mathrm{Cls}]$ : $19.5[16.8,22.3] \mu \mathrm{m})$ showed the strongest 
relationship with CIMT followed by VAT $(5.86[4.65,7.07] \mu \mathrm{m})$, SCAT $(5.00[2.85,7.15]$ $\mu \mathrm{m}), \operatorname{WC}(1.27[1.09,1.44] \mu \mathrm{m})$ and $\mathrm{HC}(1.23[1.04,1.42] \mu \mathrm{m})$. Stronger associations were observed in men than in women. We established that obesity within SSA will likely result in higher levels of atherosclerosis and promote the occurrence of cardio- and cerebrovascular events, especially in males.

Chapter 4 examined race-ethnic differences in the association of classical cardiovascular risk factors with CIMT. This individual participant data metaanalysis included data from 34,025 individuals from African, African American, Asian, European and Hispanic populations. In all race-ethnic groups, men had higher CIMT levels compared to women. Age, sex, body mass index (BMI) and systolic blood pressure (SBP) had a significant positive association with CIMT in all ethnicities at varying magnitudes. When compared to European populations, the association of age, sex and SBP with CIMT was weaker in all ethnicities. Smoking (beta coefficient [95\% Cl]: $0.39 \mathrm{~mm}[0.09,0.70])$, BMI $(0.05 \mathrm{~mm}[0.01$, $0.08])$ and glucose $(0.13 \mathrm{~mm}[0.06,0.19])$ had the strongest positive association with CIMT in the Asian population when compared to all other ethnic groups. High density lipoprotein-cholesterol (HDL-C) had significant protective effects in African American $(-0.31 \mathrm{~mm}[-0.42,-0.21])$ and African $(-0.26 \mathrm{~mm}[-0.31,-0.19])$ populations only. Differences in distribution of lifestyle risk factors as well as genetics may contribute to the differences in magnitude of atherosclerosis and CVD. These differences in the association of CVD risk factors with CIMT have implications for race-ethnic-specific primary prevention strategies and also give insights into the differential contribution of risk factors to the aetiology of CVD across populations. The greatest burden of subclinical atherosclerosis in African Americans warrants further investigation.

Part 2 has two chapters with the first chapter examining the association of microalbuminuria with CIMT and the association of microalbuminuria and CIMT with high 10-year CVD risk while the second investigated the association of cardiovascular health index with common CIMT. In chapter 5 we sought to expand the evidence on the utility of the cardiovascular health index (CVHI) introduced by the American Heart Association as a tool for screening CVD. We therefore determined its association with CIMT in under-studied populations in sub-Saharan Africa. The CVHI is a valid, accessible, simple, and translatable metric for monitoring cardiovascular health and incorporates seven cardiovascular risk factors (often termed 'life's simple 7'): smoking, dietary intake, physical activity, body mass index, blood pressure, glucose, and total cholesterol. We observed inverse associations between $\mathrm{CVHI}$ and common CIMT ( $\beta$-coefficients [95\% confidence interval]: Burkina Faso, - 6.51 [- 9.83, - 3.20]

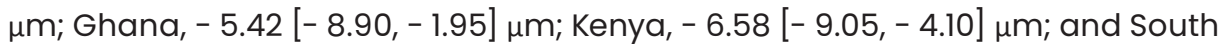
Africa, - $7.85[-9.65,-6.05] \mu \mathrm{m})$. Inverse relations were observed for women 
$(-4.44[-6.23,-2.65] \mu \mathrm{m})$ and men $(-6.27[-7.91,-4.64] \mu \mathrm{m})$ in the pooled sample. Smoking $(p<0.001)$, physical activity $(p<0.001)$, and hyperglycemia ( $<0.001$ ) were related to CIMT in women only, while blood pressure and obesity were related to CIMT in both women and men $(p<0.001)$. We demonstrated that primary prevention of atherosclerotic cardiovascular disease in this understudied population should target physical activity, smoking, obesity, hypertension, and hyperglycemia.

Chapter 6 discussed the association of microalbuminuria with CIMT and of both these variables with 10-year predicted ASCVD risk in 9011 participants. High CIMT, SUA and UACR were each associated with older age and high prevalence of both diabetes and hypertension (all associations $p<0.01$ ). The SUA was associated with higher odds of carotid atherosclerosis compared to UACR. Common CIMT, SUA and UACR were strongly associated with 10-year ASCVD risk. The CIMT had a higher odds ratio (95\% confidence interval) for 10-year ASCVD risk in both women $(1.95[1.78,2.14])$ and men $(1.73[1.55,1.93])$ compared to SUA $(1.27$ [1.12, $1.43]$ and $1.40[1.26,1.55]$, respectively) and $\operatorname{UACR}(1.30[1.10,1.54]$ and $1.30[1.15$, 1.46], respectively). This data demonstrates that microalbuminuria is associated with subclinical atherosclerosis and high 10-year ASCVD risk in middle-aged individuals from SSA and suggests that urine albumin can be used as a marker of CVD risk in population screening studies. 


\section{Samenvatting}

De ziektelast van atherosclerotische hart- en vaatziekten (HVZ) in subSahara Afrika stijgt gestaag en wereldwijde inspanningen om de morbiditeit en mortaliteit van HVZ terug te dringen vragen om het gebruik van context specifieke interventies. Beleidsvorming moet worden gebaseerd op pragmatisch onderzoek dat rekening houdt met lokale context die de etiologische relaties tussen risicofactoren en HVZ beïnvloedt.

In het begin van dit proefschrift werd een algemene achtergrond van het onderzoek besproken, met in hoofdstuk 1 een diepgaande discussie over de wereldwijde ziektelast van HVZ en de toenemende ziektelast in de SSA-regio. We hebben geobserveerd dat de toenemende ziektelast in SSA vooral veroorzaakt wordt door de verbetering van de levensverwachting en een toename in risicofactoren voor HVZ: hier is sprake van een epidemiologisch transitie. In dit hoofdstuk werd ook het gebruik van de intima-mediadikte (vaatwanddikte) van de A. carotis (CIMT) als marker voor HVZ besproken.

In deel 1 van het proefschrift werden de risicofactoren voor verhoogde CIMT in zowel SSA als andere populaties onderzocht.

In hoofdstuk 2 hebben we de associatie tussen klassieke HVZ risicofactoren en HIV met CIMT vastgesteld. De 8872 volwassenen ( $50.8 \%$ man) hadden een gemiddelde leeftijd van $50 \pm 6$ jaar met een voor leeftijd en geslacht gecorrigeerd gemiddelde CIMT van 640 $23 \mu \mathrm{m}$. Deelnemers uit Ghana en Burkina Faso hadden een hogere CIMT vergeleken met andere locaties. Leeftijd $(\beta=6.77,95 \% \mathrm{BI}[6.34,7.19] \mu \mathrm{m})$, body mass index $(17.6[12.5,22.8] \mu \mathrm{m})$, systolische bloeddruk $(7.52[6.21,8.83] \mu \mathrm{m}), \mathrm{LDL}$-cholesterol $(5.08[2.10,8.06] \mu \mathrm{m})$ en mannelijk geslacht $(10.3[4.75,15.9] \mu \mathrm{m})$ waren geassocieerd met een hogere CIMT. Roken was geassocieerd met een hogere CIMT in mannen. HDL-cholesterol (-12.2 [-17.9, -6.41] $\mu \mathrm{m})$ en alcohol consumptie (-13.5 [-19.1, -7.91] $\mu \mathrm{m})$ waren omgekeerd geassocieerd met CIMT. HIV was niet significant geassocieerd met CIMT (26.80 $(-41.40,95.01) \mu m ; p=0.441)$. Door de stijgende prevalentie van HVZ risicofactoren in SSA kunnen atherosclerotische ziekten mogelijk een grote pan-Afrikaanse epidemie worden, tenzij er preventieve maatregelen worden genomen specifiek gericht op het voorkomen van hypertensie, obesitas en roken. HIV-specifieke studies zijn nodig om meer inzicht te krijgen in het verband tussen HIV en CIMT in SSA.

Als reactie op de toenemende prevalentie van obesitas in SSA hebben we in hoofdstuk 3 het verband onderzocht tussen verschillende adipositas fenotypes en CIMT in 9010 volwassenen van middelbare leeftijd uit SSA. Mannen hadden 
hogere niveaus van visceraal vet (VAT) dan vrouwen. Vrouwen hadden daarentegen een hogere BMI, middel- en heupomtrek, en subcutaan vet (SCAT) dan mannen in elke regio behalve Burkina Faso. In de gepoolde analyse liet BMI (beta coëfficiënt [95\% BIs]: $19.5[16.8,22.3] \mu \mathrm{m}$ ) de sterkste associatie zien met CIMT gevolgd door VAT $(5.86[4.65,7.07] \mu \mathrm{m})$, SCAT $(5.00[2.85,7.15] \mu \mathrm{m})$, middelomtrek $(1.27[1.09,1.44] \mu \mathrm{m})$ en heupomtrek $(1.23[1.04,1.42] \mu \mathrm{m})$. Voor mannen waren de associaties sterker dan voor vrouwen. We hebben vastgesteld dat obesitas in SSA waarschijnlijk leidt tot een toename van atherosclerose en het optreden van HVZ bevordert, met name bij mannen.

In Hoofdstuk 4 onderzochten we raciaal-etnische verschillen in de associatie tussen klassieke HVZ risicofactoren en CIMT. Deze meta-analyse van individuele deelnemersgegevens bevatte data van 34,025 individuen van Afrikaanse, Afrikaans-Amerikaanse, Aziatische, Europeaanse en Latijns-Amerikaanse populaties. In alle raciaal-etnische groepen hadden mannen hogere CIMT waarden dan vrouwen. Leeftijd, geslacht, BMl, en systolische bloeddruk toonden een significant positieve associatie met CIMT in alle etniciteiten in verschillende groottes. Vergeleken met Europese populaties waren de associaties voor leeftijd, geslacht en systolische bloeddruk met CIMT zwakker in andere etnische groepen. Roken (beta coëfficiënt [95\% BI]: 0.39mm [0.09, 0.70]), BMI (0.05mm [0.01, $0.08])$ en glucose $(0.13 \mathrm{~mm}[0.06,0.19])$ hadden de sterkste positieve associatie met CIMT in Aziatische populaties vergeleken met andere raciaal-etnische groepen. HDL-cholesterol had een significante beschermende effect exclusief in Afrikaans-Amerikaanse $(-0.31 \mathrm{~mm}[-0.42,-0.21])$ en Afrikaanse $(-0.26 \mathrm{~mm}[-0.31$, -0.19]) populaties. Deze verschillen in de associatie van HVZ risicofactoren met CIMT hebben gevolgen voor specifieke raciaal-etnische preventiestrategieën en geven inzicht in de verschillende invloed van risicofactoren op de etiologie van HVZ over populaties. De grote ziektelast van subklinische atherosclerose bij Afro-Amerikanen vraagt om nader onderzoek.

Deel 2 heeft twee hoofdstukken waarvan in gaat op de associatie van microalbuminurie met CIMT en de associatie van microalbuminurie en CIMT met een hoog 10-jaars HVZ risico. In het tweede hoofdstuk wordt de relatie van de cardiovascular health index met CIMT onderzocht. In hoofdstuk $\mathbf{5}$ probeerden we meer bewijs te vinden voor het nut van de cardiovascular health index (CVHI) van de American Heart Association als hulpmiddel voor het screenen van HVZ. Daarvoor hebben we de associatie van de CVHI met CIMT onderzocht in onderbestudeerde populaties in SSA. De CVHI is een valide, toegankelijk, simpel en vertaalbaar meetinstrument voor het monitoren van cardiovasculaire gezondheid en bevat zeven HVZ risicofactoren (vaak 'life's simple 7' genoemd): roken, voedingsinname, lichaamsbeweging, BMI, bloeddruk, glucose en totaalcholesterol. We observeerden een omgekeerd verband tussen $\mathrm{CVHI}$ en 
CIMT ( $\beta$-coëfficiënten [95\% betrouwbaarheidsinterval]: Burkina Faso, - 6.51 [-

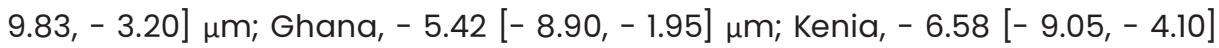
$\mu \mathrm{m}$; en Zuid-Afrika, - 7.85 [-9.65, - 6.05] $\mu \mathrm{m})$. Omgekeerde associaties werden zowel bij vrouwen $(-4.44[-6.23,-2.65] \mu \mathrm{m})$ als mannen $(-6.27$ [- 7.91, - 4.64] $\mu \mathrm{m})$ gezien in de gepoolde steekproef. Roken $(p<0.001)$, lichaamsbeweging $(p<0.001)$, en hyperglykemie $(p<0.001)$ waren alleen bij vrouwen gerelateerd aan CIMT, terwijl bloeddruk en obesitas geassocieerd waren met CIMT bij zowel mannen als vrouwen $(p<0.001)$. We hebben aangetoond dat primaire preventie van atherosclerotische HVZ in deze onderbelichte populatie gericht moet zijn op lichaamsbeweging, roken, obesitas, hypertensie en hyperglykemie.

In hoofdstuk 6 bekeken we de associatie van microalbuminurie met CIMT en van beide variabelen met het 10-jaars HVZ risico bij 9011 deelnemers. Hoge CIMT, serum urinezuur (SUA) en urine albumine/creatinine ratio (UACR) waren elk geassocieerd met hogere leeftijd en hoge prevalentie van zowel diabetes als hypertensie (alle associaties $\mathrm{p}$ <0.01). SUA was geassocieerd met een hoger risico op halsslagader atherosclerose vergeleken met UACR. Algemene CIMT, SUA en UACR waren sterk geassocieerd met 10-jaars HVZ risico. CIMT had een hogere odds ratio (95\% betrouwbaarheidsinterval) voor 10-jaars HVZ risico in zowel vrouwen $(1.95[1.78,2.14])$ als mannen $(1.73[1.55,1.93])$ vergeleken met SUA (1.27 [1.12, 1.43] en 1.40 [1.26, 1.55], respectievelijk) en UACR (1.30 [1.10, 1.54] en 1.30 $[1.15,1.46]$, respectievelijk). Dit toont aan dat microalbuminurie geassocieerd is met subklinische atherosclerose en hoog 10-jaars HVZ risico in personen van middelbare leeftijd uit SSA. Ook suggereren deze uitkomsten dat urine albumine gebruikt kan worden als marker voor HVZ risico in bevolkingsonderzoeken. 


\section{Acknowledgements}

The benefits of collaborative research are enormous and result in the generation of new knowledge or expansion of old knowledge. This PhD illustrates the benefits of collaborative research in the global health community. I wish to acknowledge individuals and groups who contributed directly and indirectly to the success of my PhD programme.

\section{My supervisory team}

I am immensely grateful to my supervisory team which is collaboration between individuals of diverse expertise and experiences. I appreciate the time, patience and sacrifices you individually made to advance this research work. Your thoughtful inputs, guidance and advice are greatly appreciated.

To Prof Dr. Diederick E. Grobbee, my main promoter, I am indebted to you for finding my proposal worthy of the Julius Global Health scholarship and for making time to discuss progress of this $\mathrm{PhD}$ thesis during my visit to Utrecht and through online meetings.

Prof Nigel J. Crowther, we've come a long way. You supervised my Master's thesis in the University of the Witwatersrand, Johannesburg, South Africa and I had no doubt we were going to have a long run on the academic turf. Your due diligence with my write up often came with a final touch of excellence. I hope we continue to collaborate in the near future in research projects or to co-supervise other students.

I probably wouldn't have pursued this PhD program without Associate Prof Kerstin Klipstein-Grobusch's recommendation and constant encouragement. Your rich experiences with research collaborations across the African continent were brought to bear in establishing this collaborative PhD work between the H3Africa AWI-Gen consortium and the Julius Center for Health Sciences and Primary Care at the University Medical Center Utrecht, Utrecht University in the Netherlands. A solid foundation has been laid and I look forward to nurturing the research collaboration further. For the social gatherings you and your husband, Prof Martin Grobusch and children shared with me and my colleagues in your home, I am most grateful.

I am particularly thankful to the director of the Navrongo Health Research Centre - Dr. Abraham R Oduro - for the various forms of support you offered me. I am deeply appreciative, "a ke enle" of your friendship and mentorship. 
I want to particularly extend a warm thank you to Prof Michiel L. Bots for bringing your rich experience in the subject area to the improvement of my overall thesis. You further enhanced this thesis with data from the USE-IMT collaboration. Your prompt responses and detail critique did indeed were indeed I look forward to working with you in the near future.

\section{The Navrongo Health Research Centre (NHRC)}

To the immediate past director (Dr. Abraham Oduro), current acting director (Dr. Patrick Ansah) and staff of the NHRC (especially Mrs. Andriana Sumboh, Mr. Jonas Dakurah, Mr. Michael Banseh), I wish to express my profound gratitude for the various contributions you made towards my PhD studies. I am particularly grateful to the Navrongo AWI-Gen study team - Eric Fato, Mrs. Immaculate Anati, Felix Ayelazoya, Deborah K. Narwortey, Mathilda Tsifodze and Enyonam Duah for zealously collected data that contributed to this study I am very grateful. I express a huge gratitude to Dr. Godfred Agongo for all the hard work in coordinating activities of the AWI-Gen study. In a special way I want to thank Dr. Cornelius Debpuur, Dr. James Akazili, Dr. Koku Awoonor-Williams, Dr. Lucas Amenga-Etego, Dr. Paulina Tindana, Dr. Evelyn Sakeah, Dr. Thomas Anyorigiya, Dr. Paul Welaga and Mr. Samuel T. Chatio for your good counsel and words of encouragement. Dr. Michael Kaburise deserves a mention here too. Thank you all for being there for me.

\section{The H3Africa AWI-Gen consortium, Johannesburg, South Africa}

I wish acknowledge Prof Michele Ramsay (PI), Prof Osman Sankoh (co-PI), site leaders and researchers in Nanoro, Nairobi, Dikgale, Agincourt, Soweto and Navrongo. I also acknowledge researchers and AWI-Gen team at the collaborative center - Sydney Brenner institute for Molecular Bioscience for the various contributions to the success of the AWI-Gen study. Thank you to Mr. Freedom Mukomana and Dr. Stuart Ali for leading all the data quality control efforts. I also commend the various community stakeholders and particularly the study participants for their cooperation in enduring long hours during the data collection. To researchers of the AWI-Gen study who co-authored some of the manuscripts, I am grateful to you for your time and academic contribution.

\section{The USE-IMT consortium}

The highlight of this thesis was the race-ethnic comparisons which was possible due to the contribution of the USE-IMT consortium data. I am most grateful to Prof Michiel L. Bots and all investigators of the individual cohorts and their sponsors for making sure these studies took place. 


\section{PhD colleagues, postdocs, administrative staff and friends}

Indeed am grateful to my PhD colleagues who over the period have grown from acquaintances to comrades and friends. To the following postdocs and assistant professors, Dr. Daniel Boateng, Dr. Alinda Vos, Dr. Joyce Browne, Dr. Mary Amoakoh-Coleman, Dr. Edward Antwi, Dr. Hannah Amoakoh-Brown, Dr. George S. Downward, Dr. Virissa C. Lenters and Dr. Salisu Ishaku, I say thank you for the intellectual and social interactions we had along the corridors of the Julius center, at meetings, seminars and in the beautiful environs of Utrecht.

To my fellow PhD colleagues of the global health program in UMCU for your friendship and collaboration, you are all appreciated: Dr. Timothy Olanrewaju, Dr. Frida Soesanti and Dr. Kwame Adu-Bonsaffoh, Mr. Mansour Alghamdi, Dr. Reuben Mutagaywa and Dr. Lulu Laiser. I look forward to future collaboration in our various fields of expertise.

I am thankful to Melanie Pero, Harold van Kesteren, Giene de Vries, Wout de Nooij, Anna Marza-Florensa, Nikki Giron and Josephine Rees for their administrative assistance during the course of my PhD studies at UMCU. A special thanks to Coby van Rijn at the Julius Centrum reception for the frequent interactions during coffee breaks at the Julius Center. I am also indebted to Vroon Flink, Elsie, and Mieke for organizing my accommodation arrangement and making sure the famous Biltstraat Houisevestting was a peaceful heaven for living and working on my thesis.

Dr. Daniel Boateng (Ghana); Dr. Alinda Vos (the Netherlands), Dr. Felix Chilunga (Malawi), Dr. Frida Soesanti (Indonesia), Mr. Mansour Alghamdi (Saudi Arabia) and Dr. Gertrude Nsormah Nyaaba (Ghana), Benedict Y. Campbell (Hague) and Severine Dery (Wageningen), you guys are indeed special friends who played a special part in this adventure. We grew from friends to family and I am deeply grateful to you for being a part of this journey.

I am indeed grateful to Dr. Anthony Agoswin Musah, Dr. Mrs. Agnes SneiderMusah and Mr. Benjamin Akatabanusa, my beloved friends in Freiburg and Bielefeld Germany who made my stay away from home worthwhile. I am tremendously grateful for your friendship. Mr. Lawrence Anedam Tiko, Mrs. Leona Tiko and family, I deeply appreciate the time you dedicated to supporting my family in my absence. You've always been there and I am indeed grateful to you. Mr. Felix Made, I wish to remind you that the journey remains "forward ever". Thanks for the time and encouragement we gave each other in our pursuit of knowledge. 
I am profoundly grateful to the family of Steven Bastian Hoek (Uncle Steve) and Aunty Monica Adrivum Akanyinte and your children Andre and Margeet in Wageningen. You are such a wonderful family and you opened your home to me. Not to talk of the countless diners I shared with your entire family - I sincerely cherish the moments. You were far too kind and good and you indeed left good and warm impressions in my heart. I am forever grateful to you. I am equally grateful to Frida Adiali and Madam Mary Ayaga (Mayaga fame) who also supported me in diverse ways.

I am grateful to the Ghana Ambassador to the Netherlands, He excellence Mr Francis Danti Kotia and family as well as Mr Michael Nyaaba Asibi of the Ghana Embassy in Belgium. I wish to also express my profound appreciation to everyone who has assisted me in diverse ways. You would agree I am unable to mention you all by your names but I am indeed grateful to you all for your immense support.

\section{Family}

I am thankful to my parents Mr. Henry Alexis N. Nonterah and Mrs. Faustina N. Nonterah and my siblings Josephine Kawiah, Margaret Anuyire, Mary Esther Yagade and Esmond Wedam Nonterah for their words of encouragement. I acknowledge my late uncle Captain Benjamin Joseph Labre Nonterah who sadly passed away on $2^{\text {nd }}$ February, 2020 when I enplaned to the Netherlands to compile my thesis. Sadly you are not here to see this day but I know you share in this moment with your maker. RIP with your maker as I continue to cherish and hold on to the memories we shared together.

Finally to my wife, Dr. Mrs. Cynthia Nonterah and my sons, Wedaga Cyrus and Kwolam Andrew Nonterah, I appreciate your patience and for enduring the numerous weeks and months of my absence during this PhD period. Your sacrifices have indeed paid off and I am most grateful to you for having made those sacrifices for me and for our family. God richly bless us! 


\section{Contributing authors (alphabetical order)}

Agongo, G.

Navrongo Health Research Centre, Ghana Health Service, Navrongo, Ghana

Alberts, $\mathrm{M}$.

Department of Pathology and Medical Sciences, DIMAMO Health and Demographic Surveillance System, University of Limpopo, South Africa

Ali, S.

Sydney Brenner Institute of Molecular Bioscience, Faculty of Health Sciences, University of the Witwatersrand, Johannesburg, South Africa

Anderson, T.J.

Department of Cardiac Sciences and Libin Cardiovascular Institute of Alberta, University of Calgary, Calgary, Alberta, Canada

Asiki, G.

African Population and Health Research Centre (APHRC), Nairobi, Kenya

Boateng, D.

Julius Global Health, Julius Center for Health Sciences and Primary Care, University Medical Center Utrecht, Utrecht University, Utrecht, the Netherlands

Bots, M.L.

Julius Global Health, Julius Center for Health Sciences and Primary Care, University Medical Center Utrecht, Utrecht University, Utrecht, the Netherlands

Boua, P. R.

Clinical Research Unit of Nanoro, Institut de Recherché en Sciences de la Santé, Nanoro, Burkina Faso

Choma, S.S.R.

Department of Pathology and Medical Sciences, DIMAMO Health and Demographic Surveillance System, University of Limpopo, South Africa

Couper, D.J.

Collaborative Studies Coordinating Center, Department of Biostatistics, University of North Carolina at Chapel Hill, USA

Crowther, N.J.

Department of Chemical Pathology, National Health Laboratory Service, Faculty of Health Sciences, University of the Witwatersrand, Johannesburg, South Africa

Debpuur, C.

Navrongo Health Research Centre, Ghana Health Service, Navrongo, Ghana 
Engström, G.

Department of Clinical Sciences in Malmö, Lund University, Skåne University Hospital, Malmö, Sweden

de Graaf, J.

Department of General Internal Medicine, Division of Vascular Medicine, Nijmegen University Medical Centre, the Netherlands

Grobbee D.E.

Julius Global Health, Julius Center for Health Sciences and Primary Care, University Medical Center Utrecht, Utrecht University, Utrecht, the Netherlands

Hazelhurst, S.

Sydney Brenner Institute of Molecular Bioscience, Faculty of Health Sciences, University of the Witwatersrand, Johannesburg, South Africa

Kahn, K.

South African Medical Research Council/Wits Rural Public Health and Health Transitions Research Unit (Agincourt), School of Public Health, Faculty of Health Sciences, University of the Witwatersrand, Johannesburg, South Africa

Kauhanen, J.

The Institute of Public Health and clinical Nutrition, School of Medicine, Faculty of Health Sciences, University of Eastern Finland (UEF), Helsinki, Finland

Klipstein-Grobusch, K.

Julius Global Health, Julius Center for Health Sciences and Primary Care, University Medical Center Utrecht, Utrecht University, Utrecht, the Netherlands

Kyobutungi, C.

African Population and Health Research Centre (APHRC), Nairobi, Kenya

Lonn, E. M.

Department of Medicine, Division of Cardiology and Population Health Research Institute, McMaster University, Hamilton, Ontario, Canada

Mashinya, $\mathrm{F}$

Department of Pathology and Medical Sciences, DIMAMO Health and Demographic Surveillance System, University of Limpopo, South Africa

Mathiessen, E. B.

Brain and Circulation Research Group, Institute of Clinical Medicine, University of Troms $\varnothing$, Tromsø, Norway

Micklesfield, L. K.

South African Medical Research Council/Developmental Pathways for Health Research Unit (DPHRU), Department of Paediatrics, School of Clinical Medicine, Faculty of Health Sciences, University of the Witwatersrand, Johannesburg, South Africa 
Mohammed, S. F.

African Population and Health Research Centre (APHRC), Nairobi, Kenya

Mukomana, F.

Sydney Brenner Institute of Molecular Bioscience, Faculty of Health Sciences, University of the Witwatersrand, Johannesburg, South Africa

Nakanabo-Diallo, S.

Clinical Research Unit of Nanoro, Institut de Recherché en Sciences de la Santé, Nanoro, Burkina Faso

Norris, S. A.

South African Medical Research Council/Developmental Pathways for Health Research Unit (DPHRU), Department of Paediatrics, School of Clinical Medicine, Faculty of Health Sciences, University of the Witwatersrand, Johannesburg, South Africa

Oduro, A. R.

Navrongo Health Research Centre, Ghana Health Service, Navrongo, Ghana

Okazaki, S.

Stroke Center, Department of Neurology, Osaka University Graduate School of Medicine, Osaka, Japan

Polak, J. F.

Department of Radiology, Tufts Medical Center, Boston, Massachusetts, USA

Raal, F. J.

Carbohydrate and Lipid Metabolism Research Unit, Faculty of Health Sciences, University of the Witwatersrand, Johannesburg, South Africa

Ramsay, M.

Sydney Brenner Institute of Molecular Bioscience, Faculty of Health Sciences, University of the Witwatersrand, Johannesburg, South Africa

Rundek, T.

University of Miami Miller School of Medicine, Miami, Florida, USA

Salonen, J. T.

MAS-Metabolic Analytical Services Oy, Helsinki, Finland

Sankoh O.

INDEPTH-Network, Accra, Ghana

Soo, C.

Sydney Brenner Institute of Molecular Bioscience, Faculty of Health Sciences, University of the Witwatersrand, Johannesburg, South Africa

Sorgho, $\mathrm{H}$.

Clinical Research Unit of Nanoro, Institut de Recherché en Sciences de la Santé, Nanoro, Burkina Faso 
Tinto, $\mathrm{H}$.

Clinical Research Unit of Nanoro, Institut de Recherché en Sciences de la Santé, Nanoro, Burkina Faso

Tollman, S. M.

South African Medical Research Council/Wits Rural Public Health and Health Transitions Research Unit (Agincourt), School of Public Health, Faculty of Health Sciences, University of the Witwatersrand, Johannesburg, South Africa

Tuomainen, T-P.

The Institute of Public Health and clinical Nutrition, School of Medicine, Faculty of Health Sciences, University of Eastern Finland (UEF), Helsinki, Finland

Wade, A. N.

South African Medical Research Council/Wits Rural Public Health and Health Transitions Research Unit (Agincourt), School of Public Health, Faculty of Health Sciences, University of the Witwatersrand, Johannesburg, South Africa 


\section{About the author}

Engelbert A. Nonterah was born on 10 ${ }^{\text {th }}$ February, 1984 in Navrongo, Ghana. He attended Notre Dame Minor Seminary School and proceeded to the School of Medicine and Health Sciences of the University for Development Studies where he studied BSc Human Biology. He then proceeded to the University of Ghana Medical School at Korle Bu, Accra where he carried out his clinical studies and graduated with a Bachelors in Medicine and Surgery (MBChB). After compulsory internship in the Eastern Regional Hospital where he undertook rotations in Paediatrics, Obstetrics and Gynecology and Surgery he proceeded to the University of Ghana Hospital in Legon, Accra, Ghana for a rotation in Internal Medicine.

Engelbert joined the Ghana Health Service and was stationed at the Navrongo Health Research Centre (NHRC) where he worked with the H3Africa AWI-Gen study. Engelbert proceeded to pursue Masters in Epidemiology (Epidemiology and Biostatistics) at the University of the Witwatersrand in Johannesburg where He worked as a research intern at the Sydney Brenner Institute of Molecular Bioscience and conducted quality control on the AWI-Gen ultrasound data.

Engelbert cultivated much interest in cardiovascular research and developed a proposal with Prof Nigel J. Crowther and Kerstin Klipstein-Grobusch which later became the focus of His PhD thesis. During the course of the PhD thesis, Engelbert supervised master's students from the University of Groningen in the Netherlands, University of the Witwatersrand in Johannesburg, South Africa and Georgetown University, Washington DC, USA. Engelbert maintained a strong presence in clinical work at the War Memorial Hospital, Navrongo, Ghana.

Engelbert held a research fellows position at the MRC Lifecource Epidemiology Unit, University of Southampton, UK from April, 2018 to December, 2020. During the course of the PhD he led, still leads, and works with several research projects in the NHRC: I. Improved nutrition preconception, during pregnancy and postdelivery (INPreP) - Phase I (April, 2018 - March, 2021; £350,000); II. Saving Brains from malnutrition (August, 2019 - March, 2020; \$50,000); III. Adolescents experiences during COVID-19 pandemic - GhanaTec19 (August, 2020 - March, 2021; £41,000); IV. INPreP Phase II - Improving nutrition among pregnant and lactating mothers through provision of bio-fortified food crops (Aril, 2021 March, 2022; £100,000); v. Phase I and II African-Wits-INDEPTH genomic (AWIGen) study (2013 - 2022) and VI. COVID-19 vaccine trials (2021) 
He is currently licensed by Ghana Medical and Dental Council as medical practitioner in Ghana in good standing. He is a member of the Public Health Association of Ghana (PHAG) and South Africa (PHASA) as well a member of the Ghana Medical Association (GMA) and the African Chapter of the Development Origins of Health and Disease (DOHaD) society.

Engelbert's hobbies include reading, traveling, playing basket ball and rendering community service. 


\section{List of publications}

\section{Published (order of publication)}

Dalaba MA, Nonterah EA, Chatio ST, Adoctor JK, Watson D, Barker M, Ward KA, Debpuur C. Culture and community perceptions on diet for maternal and child health: a qualitative study in rural northern Ghana. BMC Nutr. 2021 Jul 15; 7(1):36. doi: 10.1186/ s40795-021-00439-x.

Made F, Nonterah EA, Tlotleng N, Ntlebi $V$ and Naicker N. Ten-year risk of fatal cardiovascular disease and its association with metabolic risk factors among waste pickers in South Africa. BMC Cardiovascular Disorders. 2021; 21(1). Doi: 10.1186/s12872021-02150-y

Galaurchi A, Chatio ST, Beeri P, Oduro AR, Ofosu W, Hanson M, Newell ML, Norris SA, Ward KA, Nonterah EA*, Biesma R*, on behalf of the INPreP study group. Stakeholder perspectives on barriers and facilitators on the implementation of the 1000 Days Plus nutrition policy activities in Ghana. Int J Environ Res Public Health. 2021 May 17; 18(10):5317. Doi: 10.3390/ijerph18105317.

Agongo G, Debpuur C, Amenga-Etego L, Nonterah EA, Kaburise MB, Oduro A, Ramsay $M$, Tindana P. Community engagement and feedback of results in the H3Africa AWI-Gen project: Experiences from the Navrongo Demographic and Health Surveillance site in Northern Ghana. AAS Open Res. 2021; 19; 4:15. Doi: 10.12688/ aasopenres.13081.1.eCollection 2021.

Sakeah E, Aborigo RA, Debpuur C, Nonterah EA Oduro AR, Awoonor-Williams JK. Assessing selection procedures and roles of Community Health Volunteers and Community Health Management Committees in Ghana's Community-based Health Planning and Services program. PLoS One. 2021 May 5; 16(5):e0249332. Doi: 10.1371/ journal.pone.0249332. eCollection 2021.

Nonterah EA, Bots ML, Oduro A, Agongo G, Soo CC, Micklesfield LK, Mashinya F, Boua PR, Mohamed SF, Wade AN, Kyobutungi C, Tinto H, Norris SA, Tollman SM, Ramsay M, Grobbee DE, Klipstein-Grobusch K, Crowther NJ; AWI-Gen and the H3Africa Consortium. Adiposity Phenotypes and Subclinical Atherosclerosis in Adults from Sub-Saharan Africa: An H3Africa AWI-Gen Study. Glob Heart. 2021; 19; 16(1):19. Doi: $10.5334 / g h .863$.

Nonterah EA, Crowther NJ, Oduro A, Agongo G, Micklesfield LK, Boua PR, Choma SSR, Mohamed SF, Sorgho H, Tollman SM, Norris SA, Raal FJ, Grobbee DE, Ramsay M, Bots ML, Klipstein-Grobusch K; as part of the H3Africa AWI-Gen study. Poor cardiovascular health is associated with subclinical atherosclerosis in apparently healthy subSaharan African populations: an H3Africa AWI-Gen study. BMC Med. 2021; 10; 19(1):30. Doi: 10.1186/s12916-021-01909-6.

Wagner RG, Crowther NJ, Micklesfield LK, Boua PR, Nonterah EA, Mashinya F, Mohamed SF, Asiki G, Tollman S, Ramsay M, Davies Jl. Estimating the burden of cardiovascular risk in community dwellers over 40 years old in South Africa, Kenya, Burkina Faso and Ghana. BMJ Glob Health. 2021 Jan; 6(1):e003499. Doi: 10.1136/bmjgh-2020-003499. 
Agongo G, Amenga-Etego L, Nonterah EA, Debpuur C, Choudhury A, Bentley AR, Oduro AR, Rotimi CN, Crowther NJ, Ramsay M; AWI-Gen and H3Africa, H Africa. Candidate Gene Analysis Reveals Strong Association of CETP Variants with High Density Lipoprotein Cholesterol and PCSK9 Variants with Low Density Lipoprotein Cholesterol in Ghanaian Adults: An AWI-Gen Sub-Study. Front Genet. 2020 Oct 30; 11:456661. Doi: 10.3389/fgene.2020.456661. eCollection 2020.

Debpuur C, Nonterah EA Chatio ST, Adoctor JK, Dambayi E, Beeri P, Nonterah EW, AyiBisah D, Watson D, Kehoe SH, Dalaba MA, Ofosu W, Aborigo R, Welaga P, Oduro AR, Newell ML, Barker M; INPreP Study Group. Supporting maternal and child nutrition: views from community members in rural Northern Ghana. Public Health Nutr. 2020 Sep 25:1-8. doi: 10.1017/S136898002000302X. Online ahead of print.

Watson D, Kehoe SH, Erzse A, Compaoré A, Debpuur C, Nonterah EA Sorgho H, Norris SA, Hofman KJ, Lawrence W, Newell ML, Godfrey KM, Ward KA, Barker M; INPreP group. Public Health Nutr. 2020 Sep 15:1-9. Doi: 10.1017/S1368980020003018. Online ahead of print.

Haykin LA, Francke JA, Abapali A, Yakubu E, Dambayi E, Jackson EF, Aborigo R, Awuni D, Nonterah EA, Oduro AR, Bawah AA, Phillips JF, Heller DJ. Adapting a nurse-led primary care initiative to cardiovascular disease control in Ghana: a qualitative study. BMC Public Health. 2020 May 24; 20(1):745. Doi: 10.1186/s12889-020-08529-4.

Agongo G, Nonterah EA, Amenga-Etego L, Debpuur C, Kaburise MB, Ali SA, Crowther NJ, Ramsay M, Oduro AR. Blood Pressure Indices and Associated Risk Factors in a Rural West African Adult Population: Insights from an AWI-Gen sub study in Ghana. Int J Hypertens. 2020 Apr 26; 2020:4549031. Doi: 10.1155/2020/4549031. eCollection 2020.

Nonterah EA, Atindama S, Achumbowina E, Kaburise MB, Saanwie E, Ewura A, Majeedallahi Al-Hassan. Isolated Jejunal Perforation and Mesentery Injury following a Kick on the Abdomen of a College Student: A Case Report from a District Hospital in Northern Ghana. Case Reports in Critical Care. 2020; 2020:1-4. https://doi. org/10.1155/2020/3063472

Akpa OM, Made F, Ojo A, Ovbiagele B, Adu D, Motala AA, Mayosi BM, Adebamowo SN, Engel ME, Tayo B, Rotimi C, Salako B, Akinyemi R, Gebregziabher M, Sarfo F, Wahab K, Agongo G, Alberts M, Ali SA, Asiki G, Boua RP, Gómez-Olivé FX, Mashinya F, Micklesfield L, Mohamed SF, Nonterah EA, Norris SA, Sorgho H, Tollman S, Parekh RS, Chishala C, Ekuro K, Waddy SP, Peprah E, Mensah GA, Wiley K, Troyer J, Ramsay M, Owolabi $\mathrm{MO}$, as members of the CVD Working Group of the H3Africa Consortium. Regional patterns and association between obesity and hypertension in Africa: Evidence from the H3Africa CHAIR Study. Hypertension. 2020 May; 75(5):1167-1178. Doi: 10.1161/ HYPERTENSIONAHA.119.14147. Epub 2020 Mar 16.

Nonterah EA, Agorinya IA, Kanmiki EW, Kagura J, Tamimu M, Ayamba EY, Esmond W. Nonterah, Michael B. Kaburise, Majeedallahi Al-Hassan, Winfred Ofosu, Abraham R. Oduro, John K. Awonoor-Williams. Trends and risk factors associated with stillbirths: A case study of the Navrongo War Memorial Hospital in Northern Ghana. PLoS One. 2020 Feb 21; 15(2):e0229013. Doi: 10.1371/journal.pone.0229013. eCollection 2020. 
Boua PR, Brandenburg J-T, Choudhury A, Hazelhurst S, Sengupta D, Agongo G, Nonterah EA, Oduro AR, Tinto H, Mathew CG, Sorgho H and Ramsay M (2020). Novel and known gene-smoking interactions with cIMT identified as potential drivers for atherosclerosis risk in West-African Populations of the AWI-Gen study. Front Genet. 2020 Feb 7; 10:1354. Doi: 10.3389/fgene.2019.01354. eCollection 2019.

Nonterah EA, Boua PR, Klipstein-Grobusch K, Asiki G, Micklesfield LK, Agongo G, Ali SA, Mashinya F, Sorgho $H$, Nakanabo-Diallo $S$, Debpuur C, Kyobutungi C, Alberts $M$, Norris S, Tollman S, Tinto H, Soo CC, Mukomana F, Hazelhurst S, Wade AN, Kahn K, Oduro AR, Grobbee DE, Sankoh O, Ramsay M, Bots ML, Crowther NJ; as members and collaborators of AWI-Gen and the H3Africa Consortium.. Classical cardiovascular risk factors and HIV are associated with carotid intima-media thickness in adults from sub-Saharan Africa: Findings from H3Africa AWI-Gen Study. J Am Heart Assoc. 2019 Jul 16; 8(14):e011506. doi: 10.1161/JAHA.118.011506. Epub 2019 Jul 13.

Nonterah EA, Kanmiki EW, Agorinya IA, Sakeah E, Tamimu M, Kagura J, Kaburise MB, Ayamba EY, Nonterah EW, Awuni D, Al-Hassan M, Ofosu Winfred, Awoonor-Williams JK and Oduro AR. Prevalence and adverse obstetric outcomes of female genital mutilation among women in rural Northern Ghana. Eur J Public Health. 2020 Jun I; 30(3):561-567. Doi: 10.1093/eurpub/ckz195.

Nonterah EA, Adomolga E, Yidana A, Kagura J, Agorinya I, Ayamba EY, Atindama S, Kaburise $\mathrm{MB}, \mathrm{Al}-\mathrm{Hassan} \mathrm{M}$. Descriptive epidemiology of anaemia among pregnant women initiating antenatal care in rural Northern Ghana. Afr J Prim Health Care Fam Med. 2019 Apr 10; 11(1):el-e7. Doi: 10.4102/phcfm.v1li1.1892.

Ramsay M, Crowther NJ, Agongo G, Ali SA, Asiki G, Boua RP, Gómez-Olivé FX, Kahn K, Khayeka-Wandabwa C, F. Mashinya, L. Micklesfield, F. Mukomana, Nonterah EA, Soo C, Sorgho H, Wade AN, Wagner RG, Alberts M, Hazelhurst S, Kyobutungi C, Norris SA, Oduro AR, Sankoh O, Tinto H, Tollman $S$ as members of AWI-Gen and the H3Africa Consortium (2018) Regional and sex-specific variation in BMI distribution in four subSaharan African countries: The H3Africa AWI-Gen study. Glob Health Action. 2018; 11(sup2):1556561. Doi: 10.1080/16549716.2018.1556561.

Agongo G, Nonterah EA, Debpuur C, Amenga-Etego L, Ali S, Oduro A, Crowther N, Ramsay $\mathrm{M}$ and as members of AWI-Gen and the H3Africa Consortium. The burden of dyslipidaemia and factors associated with lipid levels among adults in rural northern Ghana: an AWI-Gen sub-study. PLoS One 13(11): e0206326. https://doi.org/10.1371/ journal.pone.0206326

Agorinya IA, Kanmiki EW, Nonterah EA, Tediosi F, Akazili J, Welaga P, Azongo D, Oduro RA. Socio-demographic determinants of low birth weight: Evidence from the Kassena-Nankana districts of the Upper East Region of Ghana. PLoS One. 2018 NoV 14; 13(11):e0206207. Doi: 10.1371/journal.pone.0206207. eCollection 2018.

Richter LM, Mathews S, Kagura J, Nonterah E. A longitudinal perspective on violence in the lives of South African children from the Birth to Twenty Plus cohort study in Johannesburg-Soweto. S Afr Med J. 2018 Feb 27; 108(3):181-186. Doi: 10.7196/SAMJ.2018. v108i3.12661. 
Richter LM, Mathews S, Nonterah $\mathbf{E}_{\mathbf{1}}$ Masilela L. A longitudinal perspective on boys as victims of childhood sexual abuse in South Africa: Consequences for adult mental health. Child Abuse Negl. 2018 Oct; 84:1-10. Doi: 10.1016/j.chiabu.2018.07.016. Epub 2018 Jul 20.

Ali SA, Soo C, Agongo G, Alberts M, Amenga-Etego L, Boua RP, Choudhury A, Crowther NJ, Debpuur C, Gómez-Olivé FX, Guiraud I, Haregu TN, Hazelhurst S, Kahn K, Khayeka-Wandabwa C, Kyobutungi C, Lombard Z, Mashinya F, Micklesfield L, Mohamed SF, Mukomana F, Nakanabo-Diallo S, Natama HM, Ngomi N, Nonterah EA Norris SA, Oduro AR, Somé AM, Sorgho H, Tindana P, Tinto H, Tollman S, Twine R, Wade A, Sankoh O \& Ramsay M. (2018) Genomic and environmental risk factors for cardiometabolic diseases in Africa: methods used for Phase 1 of the AWI-Gen population cross sectional study. Glob Health Action. 2018; 11(sup2):1507133. Doi: 10.1080/16549716.2018.1507133.

Nonterah EA, Debpuur C, Agongo G, Amenga-Etego L, Crowther N, Ramsay M, Abraham R. Oduro. Socio-demographic and behavioural determinants of body mass index among an adult population in rural Northern Ghana: The AWI-Gen study. Glob Health Action. 2018; 11(sup2):1467588. Doi: 10.1080/16549716.2018.1467588.

Gómez-Olivé FX, Ali SA, Made F, Kyobutungi C, Nonterah E, Micklesfield L, Alberts M, Boua R, Hazelhurst S, Debpuur C, Mashinya F, Dikotope S, Sorgho H, Cook I, Muthuri S, Soo C, Mukomana F, Agongo G, Wandabwa C, Afolabi S, Oduro A, Tinto H, Wagner RG, Haregu T, Wade A, Kahn K, Norris SA, Crowther NJ, Tollman S, Sankoh O, Ramsay $\mathrm{M}$; as members of AWI-Gen and the H3Africa Consortium. Stark regional and sex differences in the prevalence and awareness of hypertension across six sites in sub-Saharan Africa: an H3Africa AWI-Gen study. Glob Heart. 2017 Jun; 12 (2):81-90. Doi: 10.1016/j.gheart.2017.01.007. Epub 2017 Mar 13

Ramsay M, Crowther N, Tambo E, Agongo G, Baloyi V, Dikotope S, Gómez-Olivé X, Jaff $\mathrm{N}$, Sorgho H, Wagner R, Khayeka-Wandabwa C, Choudhury A, Hazelhurst S, Kahn K, Lombard Z, Mukomana F, Soo C, Soodyall H, Wade A, Afolabi S, Agorinya I, AmengaEtego L, Ali SA, Bognini JD, Boua RP, Debpuur C, Diallo S, Fato E, Kazienga A, Konkobo SZ, Kouraogo PM, Mashinya F, Micklesfield L, Nakanabo-Diallo S, Njamwea B, Nonterah E, Ouedraogo S, Pillay $V$, Somande AM, Tindana $P$, Twine R, Alberts $M$, Kyobutungi $C$, Norris SA, Oduro AR, Tinto H, Tollman S and Sankoh $O$ as members of AWI-Gen and the H3Africa Consortium. H3Africa AWI-Gen Collaborative Centre: a resource to study the interplay between genomic and environmental risk factors for cardiometabolic diseases in four sub-Saharan African countries. Glob Health Epidemiol Genom. 2016 Nov 22; 1:e20. Doi: 10.1017/gheg.2016.17. eCollection 2016. 


\section{Submitted manuscripts}

Nonterah EA Crowther NJ, Klipstein-Grobusch K, Oduro AR, Kavousi M, Agongo G, Anderson TJ, Asiki G, Boua PR, Choma SSR, Couper DJ, Engström G, de Graaf J, Kauhanen J, Lonn EM, Mathiessen EB, Micklesfield LK, Okazaki S, Polak JF, Rundek T, Salonen JT, Tollman SM, Tuomainen T-P, Grobbee DE, Ramsay M, and Bots ML, for the H3Africa AWI-Gen and USE-IMT collaborative study group. Race-ethnic differences in the association between classical cardiovascular risk factors and common carotid intima-media thickness: an individual participant data meta-analysis

Nonterah EA, Boateng $D$, Crowther NJ, Klipstein-Grobusch K, Oduro AR, Agongo G, Mohamed SF, Boua PR, Choma SSR, Norris SA, Tollman SM, Bots ML, Ramsay M and Diederick Grobbee, for the H3Africa AWI-Gen study. Carotid atherosclerosis, microalbuminuria and predicted 10-year cardiovascular disease risk in sub-Saharan Africa

Nonterah EA, Welaga P, Chatio ST, Kehoe SH, Ofosu W, Ward KA, Godfrey KM, Newell $M-L$ and Oduro AR as members of the INPreP study group. Children born during the hunger season are at a higher risk of severe acute malnutrition: findings from a Guinea Sahelian ecological zone in northern Ghana

Choudhury A, Brandenburg JT, Chikowore T, Sengupta D, Boua PR, Crowther NJ, Agongo G, Asiki G, Gómez-Olivé FX, Kisiangani I, Maimela E, Masemola-Maphuta M, Micklesfield LK, Nonterah EA, Norris SA, Sorgho H, Tinto H, Tollman SM, Graham S, Willer C, Hazelhurst S, Ramsay M. Meta-analysis of 25,000 continental Africans provides insights into the genetic architecture of lipid traits

Boua PR, Brandenburg JT, Choudhury A, Sorgho H, Nonterah EA, Agongo G, Asiki G, Micklesfield LK, Choma SSR, Gómez-Olivé FX, Hazelhurst S, Tinto H, Crowther NJ, Mathew CG, Ramsay Mas members of AWI-Gen and the H3Africa Consortium. Genetic associations with carotid intima-media thickness link to atherosclerosis biology with sex-specific effects in sub-Saharan Africans

Dalaba M*, Nonterah EA*, Chatio ST, Adoctor JK, Dambayi E, Nonterah EW, Stephen Azalia S, Ayi-Bisah D, Erzse A, Watson D, Hardy-Johnson P, Kehoe SH, Tugendhaft A, Ward K, Debpuur C, Oduro AR, Ofosu W, Danis M, and Barker M on behalf of the INPreP study group. Engaging community members in priority setting for nutrition interventions in rural northern Ghana

Adoctor JK, Chatio ST, Nonterah EA, Kehoe SH, Dalaba MA, Dambayi E, Nonterah EW, Debpuur C, Oduro AR, Ofosu W, Barker M, Ward K. Perceived infant feeding practices and suitable local foods to improve health in the first 1000days of life in rural KassenaNankana Districts of Northern Ghana

Oladokun ST, Nonterah EA, Chirwa T, Oduro AR, Welaga P. Cause specific under-five mortality rates and associated risk factors among children in northern Ghana between 2007 and 2012: A longitudinal analysis 


